\title{
Late-Stage Diversification of Biarylphosphines through Rhodium(I)-Catalyzed C-H Bond Alkenylation with Internal Alkynes
}

Zhuan Zhang, Marie Cordier, Pierre H. Dixneuf, Jean-François Soulé Univ Rennes, CNRS, ISCR UMR 6226, F-35000 Rennes, France

Email: jean-francois.soule@univ-rennes1.fr

\section{Table of Contents}

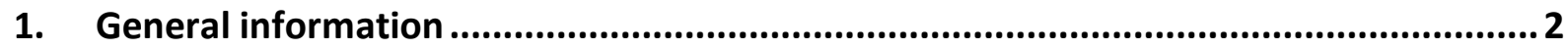

2. $\mathrm{Rh}(\mathrm{I})$-Catalyzed $\mathrm{C}-\mathrm{H}$ Bond Alkenylation: Procedure and Compound Characterizations 2

3. Rh(I)-Catalyzed C-H Bond Alkenylation: Reaction on $1 \mathrm{mmol}$ Scale ............................16

4. Preparation and Characterization of the Palladium Complex 5a ................................. 17

4. Catalytic Evaluation of Phosphine in Pd-Catalyzed Amidation: Procedure and

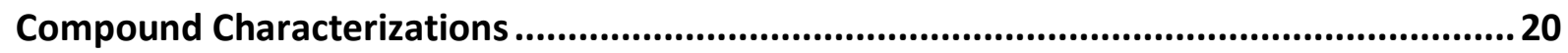

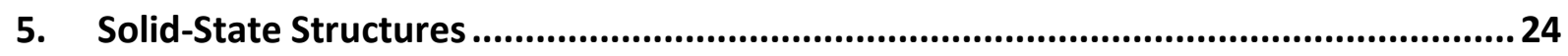

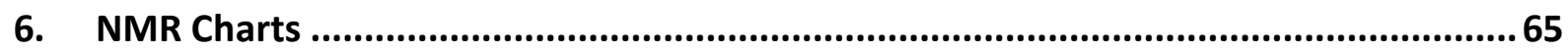

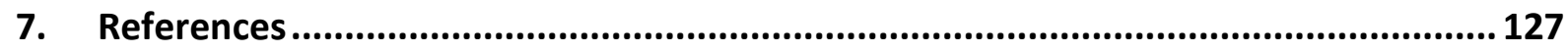




\section{General information}

All reactions were carried out under argon atmosphere with standard Schlenk techniques. All reagents were obtained from commercial sources and used as supplied. All reactions were performed using dry and degassed solvents unless stated otherwise. Toluene was purified by passage through activated alumina using a Glass Contour Solvent Dispensing System. Technical grade heptane and diethyl ether were used for column chromatography.

${ }^{1} \mathrm{H}$ NMR spectra were recorded on Bruker GPX $(300,400$ or $500 \mathrm{MHz})$ spectrometer. Chemical shifts (ठ) were reported in parts per million relative to residual chloroform (7.28 ppm for ${ }^{1} \mathrm{H} ; 77.23 \mathrm{ppm}$ for

${ }^{13} \mathrm{C}$ ), constants were reported in Hertz. ${ }^{1} \mathrm{H}$ NMR assignment abbreviations were the following: singlet $(s)$, doublet (d), triplet (t), quartet (q), doublet of doublets (dd), doublet of triplets (dt), and multiplet (m). ${ }^{13} \mathrm{C}$ NMR spectra were recorded at $100 \mathrm{MHz}$ on the same spectrometer and reported in ppm.

GC-MS analyses were performed with a GCMS-QP2010S (Shimadzu) instrument with a GC-2010 equipped with a $30 \mathrm{~m}$ capillary column (Supelco, SLBTM- 5ms, fused silica capillary column, $30 \mathrm{~m} \times 0.25$ $\mathrm{mm} \times 0.25 \mathrm{~mm}$ film thickness), which was used with helium as the vector gas. The following GC

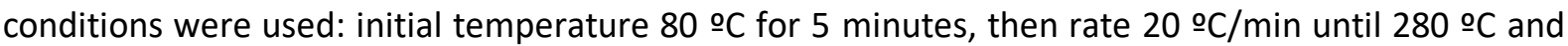
280 으 for 28 minutes.

HRMS were recorded on a Waters Q-Tof 2 mass spectrometer at the corresponding facilities of the CRMPO, Centre Régional de Mesures Physiques de l'Ouest, Université de Rennes 1.

$[\mathrm{Rh}(\mathrm{OAc})(\mathrm{COD})]_{2}$ was prepared according to literature procedures. ${ }^{[1]}$

Diphenylacetylene and Bis(4-bromophenyl)acetylene were purchased and used as received. Other diaryl alkynes were prepared according to literature procedures. ${ }^{[2]}$

\section{Rh(I)-Catalyzed C-H Bond Alkenylation: Procedure and Compound Characterizations}

General Procedure A (monoalkenylation): To a $15 \mathrm{~mL}$ oven dried Schlenk tube, KOAc (4.92 mg, 0.05 mmol, 0.25 equiv.), biarylphosphine derivative $(0.2 \mathrm{mmol}, 1$ equiv.), toluene $(1 \mathrm{~mL})$, alkyne $(0.2 \mathrm{mmol}$, 1 equiv) and $[\mathrm{Rh}(\mathrm{OAc})(\mathrm{COD})]_{2}(2.3 \mathrm{mg}, 0.004 \mathrm{mmol}, 2 \mathrm{~mol} \%)$ were successively added. The reaction mixture was evacuated by vacuum-argon cycles ( 5 times) and stirred at $120^{\circ} \mathrm{C}$ (oil bath temperature) for 24 hours. After cooling the reaction at room temperature and concentration, the crude mixture was purified by silica column chromatography to afford the desired alkenylated phosphines.

General Procedure B (symmetrical dialkenylaion): To a $15 \mathrm{~mL}$ oven dried Schlenk tube, KOAc (9.84 $\mathrm{mg}, 0.1 \mathrm{mmol}, 0.5$ equiv.), biarylphosphine derivative $(0.2 \mathrm{mmol}, 1$ equiv.), toluene ( $1 \mathrm{~mL})$, alkyne (0.6 
mmol, 3 equiv.) and $[\mathrm{Rh}(\mathrm{OAc})(\mathrm{COD})]_{2}(4.6 \mathrm{mg}, 0.008 \mathrm{mmol}, 4 \mathrm{~mol} \%)$ were successively added. The reaction mixture was evacuated by vacuum-argon cycles (5 times) and stirred at $120{ }^{\circ} \mathrm{C}$ (oil bath temperature) for 24 hours. After cooling the reaction at room temperature and concentration, the crude mixture was purified by silica column chromatography to afford the desired alkenylated phosphines.<smiles>O=C(c1ccccc1)c1ccccc1-c1ccccc1</smiles>

(E)-Dicyclohexyl(2'-(1,2-diphenylvinyl)-[1,1'-biphenyl]-2-yl)phosphane (3aa). Following the general procedure A using 2-(dicyclohexylphosphino)biphenyl (70.1 $\mathrm{mg}, 0.2 \mathrm{mmol}$ ) and diphenylacetylene $(36.1 \mathrm{mg}, 0.2 \mathrm{mmol})$, the residue was purified by flash chromatography on silica gel (heptane- $\mathrm{Et}_{2} \mathrm{O}=99: 1$ ) to afford 3aa (90.8 $\mathrm{mg}, 86 \%$ ) as a white solid; $\mathrm{mp}=170-172{ }^{\circ} \mathrm{C}$. Crystallization by diffusion method from $\mathrm{CH}_{2} \mathrm{Cl}_{2}$ solution with $n$-hexane has afforded single crystal to analyze by X-ray diffraction.

${ }^{1} \mathrm{H}$ NMR $\left(\mathrm{CDCl}_{3}, 400 \mathrm{MHz}\right): \delta 7.50-7.33(\mathrm{~m}, 4 \mathrm{H}), 7.23-7.05(\mathrm{~m}, 8 \mathrm{H}), 7.05-6.95(\mathrm{~m}, 3 \mathrm{H}), 6.92(\mathrm{~d}, J=$ $7.5 \mathrm{~Hz}, 2 \mathrm{H}), 6.75(\mathrm{dd}, J=7.8,3.8 \mathrm{~Hz}, 1 \mathrm{H}), 6.68(\mathrm{~s}, 1 \mathrm{H}), 1.89(\mathrm{~d}, J=13.0 \mathrm{~Hz}, 2 \mathrm{H}), 1.80-1.61(\mathrm{~m}, 7 \mathrm{H}), 1.52$ $(\mathrm{d}, J=8.1 \mathrm{~Hz}, 2 \mathrm{H}), 1.47-1.34(\mathrm{~m}, 3 \mathrm{H}), 1.28-0.92(\mathrm{~m}, 8 \mathrm{H})$.

${ }^{13} \mathrm{C}\left\{{ }^{1} \mathrm{H}\right\}$ NMR $\left(\mathrm{CDCl}_{3}, 101 \mathrm{MHz}\right): \delta 149.4(\mathrm{~d}, J=30.7 \mathrm{~Hz}), 144.0(\mathrm{~d}, J=1.9 \mathrm{~Hz}), 142.7,141.9(\mathrm{~d}, J=5.4 \mathrm{~Hz})$, $141.1,137.4,134.3$ (d, $J=19.8 \mathrm{~Hz}), 132.2(\mathrm{~d}, J=3.2 \mathrm{~Hz}), 132.1$ (d, $J=2.3 \mathrm{~Hz}), 131.6,130.6$ (d, $J=5.7$ $\mathrm{Hz}), 130.4,130.0(\mathrm{~d}, J=1.7 \mathrm{~Hz}), 129.4,127.7(\mathrm{~d}, J=17.0 \mathrm{~Hz}), 127.4,127.2,126.6(\mathrm{~d}, J=12.4 \mathrm{~Hz})$, $126.3,125.8,36.7(\mathrm{~d}, J=16.2 \mathrm{~Hz}), 32.8(\mathrm{~d}, J=12.8 \mathrm{~Hz}), 31.0(\mathrm{~d}, J=13.9 \mathrm{~Hz}), 30.5(\mathrm{~d}, J=19.8 \mathrm{~Hz}), 30.2$ (d, $J=13.3 \mathrm{~Hz}$ ), $29.1(\mathrm{~d}, J=3.8 \mathrm{~Hz}), 27.8(\mathrm{~d}, J=6.6 \mathrm{~Hz}), 27.7,27.3,27.1(\mathrm{~d}, J=4.2 \mathrm{~Hz}), 27.0,26.5(\mathrm{~d}, J$ $=11.9 \mathrm{~Hz})$.

${ }^{31} \mathrm{P}\left\{{ }^{1} \mathrm{H}\right\}$ NMR $\left(\mathrm{CDCl}_{3}, 162 \mathrm{MHz}\right): \delta-10.5$ (s).

HRMS (ESI) m/z: [M+H] $]^{+}$Calcd for $\mathrm{C}_{38} \mathrm{H}_{42} \mathrm{P}$ 529.3018; Found 529.3022.<smiles>Pc1ccccc1-c1ccccc1-c1ccccc1P</smiles>
$\mathrm{mg}, 0.2 \mathrm{mmol}$ ) and diphenylacetylene $(36.1 \mathrm{mg}, 0.2 \mathrm{mmol})$, the residue was purified by flash chromatography on silica gel (heptane-Et $\mathrm{t}_{2} \mathrm{O}=99: 1$ ) to afford 3 ba (78.5 $\mathrm{mg}, 76 \%$ ) as a white solid; $\mathrm{mp}=173-175^{\circ} \mathrm{C}$.

${ }^{1} \mathrm{H}$ NMR $\left(\mathrm{CDCl}_{3}, 400 \mathrm{MHz}\right): \delta 7.50(\mathrm{dd}, J=7.7,1.3 \mathrm{~Hz}, 1 \mathrm{H}), 7.43-7.16(\mathrm{~m}, 10 \mathrm{H}), 7.15-6.88(\mathrm{~m}, 14 \mathrm{H})$, $6.85-6.81(\mathrm{~m}, 2 \mathrm{H}), 6.80(\mathrm{~s}, 1 \mathrm{H}), 6.73(\mathrm{~d}, J=7.6 \mathrm{~Hz}, 1 \mathrm{H})$.

${ }^{13} \mathrm{C}\left\{{ }^{1} \mathrm{H}\right\}$ NMR $\left(\mathrm{CDCl}_{3}, 101 \mathrm{MHz}\right): \delta 147.5(\mathrm{~d}, J=31.7 \mathrm{~Hz}), 143.5,143.1,140.6(\mathrm{~d}, J=6.6 \mathrm{~Hz}), 140.5,138.6$ ( $d, J=13.1 \mathrm{~Hz}), 137.4,136.1$ (d, $J=11.7 \mathrm{~Hz}), 134.1,133.9,133.7$ (d, $J=1.9 \mathrm{~Hz}), 133.4,133.2,131.7$ (d, $J=2.5 \mathrm{~Hz}$ ), 131.3 (d, J = 3.9 Hz), 130.3 (d, J = 2.3 Hz), 129.7, $129.4,128.5,128.3(\mathrm{~d}, J=7.0 \mathrm{~Hz})$, $128.1(\mathrm{~d}, J=6.0 \mathrm{~Hz}), 127.8(\mathrm{~d}, J=5.3 \mathrm{~Hz}), 127.5,126.91,126.5(\mathrm{~d}, J=3.1 \mathrm{~Hz}), 126.5$. 
${ }^{31} \mathrm{P}\left\{{ }^{1} \mathrm{H}\right\}$ NMR $\left(\mathrm{CDCl}_{3}, 162 \mathrm{MHz}\right): \delta-13.2(\mathrm{~s})$.

HRMS (ESI) Calcd for: $\mathrm{C}_{38} \mathrm{H}_{30} \mathrm{P}: 517.2079$; Found: $517.2080[\mathrm{M}+\mathrm{H}]^{+}$<smiles>O=[PH](=O)(O)c1ccccc1-c1ccccc1C(=Cc1ccccc1)c1ccccc1</smiles>

(E)-(2'-(1,2-Diphenylvinyl)-[1,1'-biphenyl]-2-yl)diisopropylphosphane (3ca). Following the general procedure $\mathbf{A}$ using 2-(di-(iso-propyl)phosphino)biphenyl (54.1 $\mathrm{mg}, 0.2 \mathrm{mmol}$ ) and diphenylacetylene $(36.1 \mathrm{mg}, 0.2 \mathrm{mmol})$, the residue afford 3ca $(72.6 \mathrm{mg}, 81 \%)$ as a white solid; $\mathrm{mp}=148-150{ }^{\circ} \mathrm{C}$.

${ }^{1} \mathrm{H}$ NMR $\left(\mathrm{CDCl}_{3}, 400 \mathrm{MHz}\right): \delta 7.47-7.31(\mathrm{~m}, 4 \mathrm{H}), 7.18-6.91(\mathrm{~m}, 11 \mathrm{H}), 6.84(\mathrm{~s}, 1 \mathrm{H}), 6.82(\mathrm{~s}, 1 \mathrm{H}), 6.67$ (ddd, $J=7.7,3.8,1.3 \mathrm{~Hz}, 2 \mathrm{H}$ ), $2.36-1.73(\mathrm{~m}, 2 \mathrm{H}), 1.22$ (dd, $J=14.8,6.7 \mathrm{~Hz}, 3 \mathrm{H}), 1.04-0.86(\mathrm{~m}, 9 \mathrm{H})$. ${ }^{13} \mathrm{C}\left\{{ }^{1} \mathrm{H}\right\}$ NMR $\left(\mathrm{CDCl}_{3}, 101 \mathrm{MHz}\right): \delta 148.9(\mathrm{~d}, J=29.1 \mathrm{~Hz}), 144.0(\mathrm{~d}, J=1.9 \mathrm{~Hz}), 142.9,141.0,137.4,132.1$ (d, $J=2.1 \mathrm{~Hz}), 131.8,131.5,130.6,130.5,130.5,129.8(\mathrm{~d}, J=1.7 \mathrm{~Hz}), 129.4,127.7(\mathrm{~d}, J=8.2 \mathrm{~Hz})$, 127.5 , $127.4,127.3,126.6,126.4$ (d, J = 7.4 Hz), 125.9, 26.2 (d, J = 17.3 Hz), 22.6 (d, J = 10.6 Hz), 21.0 (d, $J=15.7 \mathrm{~Hz}$ ), 20.3 (d, $J=14.5 \mathrm{~Hz}), 20.0,19.1$ (d, $J=6.1 \mathrm{~Hz}$ ).

${ }^{31} \mathrm{P}\left\{{ }^{1} \mathrm{H}\right\}$ NMR $\left(\mathrm{CDCl}_{3}, 162 \mathrm{MHz}\right): \delta-2.9(\mathrm{~s})$.

HRMS (ESI) m/z: [M+H] $]^{+}$Calcd for $\mathrm{C}_{32} \mathrm{H}_{34} \mathrm{P}: 449.2392$; Found 449.2396 .<smiles>CCCCc1ccccc1-c1ccccc1C(=Cc1ccccc1)c1ccccc1</smiles>

(E)-Di-tert-butyl(2'-(1,2-diphenylvinyl)-[1,1'-biphenyl]-2-yl)phosphane (3da). Following the general procedure $\mathbf{A}$ using 2-(di-(tert-butyl)phosphino)biphenyl $(60.4 \mathrm{mg}, 0.2 \mathrm{mmol}$ ) and diphenylacetylene $(36.1 \mathrm{mg}, 0.2 \mathrm{mmol})$, the residue was purified by flash chromatography on silica gel (heptane- $\mathrm{Et}_{2} \mathrm{O}=99: 1$ ) to afford 3da (51.5 $\mathrm{mg}, 54 \%)$ as a white solid; $\mathrm{mp}=166-168^{\circ} \mathrm{C}$.

${ }^{1} \mathrm{H} \mathrm{NMR}\left(\mathrm{CDCl}_{3}, 400 \mathrm{MHz}\right): \delta 7.66(\mathrm{dt}, J=7.8,1.6 \mathrm{~Hz}, 1 \mathrm{H}), 7.39-7.28(\mathrm{~m}, 3 \mathrm{H}), 7.14-7.02(\mathrm{~m}, 8 \mathrm{H}), 6.94$ $(\mathrm{dd}, J=6.9,2.9 \mathrm{~Hz}, 2 \mathrm{H}), 6.91-6.84(\mathrm{~m}, 3 \mathrm{H}), 6.65(\mathrm{~s}, 1 \mathrm{H}), 6.62-6.54(\mathrm{~m}, 1 \mathrm{H}), 1.29(\mathrm{~d}, J=11.6 \mathrm{~Hz}, 9 \mathrm{H})$, $1.05(\mathrm{~d}, J=11.2 \mathrm{~Hz}, 9 \mathrm{H})$.

${ }^{13} \mathrm{C}\left\{{ }^{1} \mathrm{H}\right\}$ NMR $\left(\mathrm{CDCl}_{3}, 101 \mathrm{MHz}\right): \delta 149.7(\mathrm{~d}, J=33.8 \mathrm{~Hz}), 143.8(\mathrm{~d}, J=2.4 \mathrm{~Hz}), 143.1,142.4(\mathrm{~d}, J=5.7 \mathrm{~Hz})$, $141.1,137.3,135.7(\mathrm{~d}, J=27.9 \mathrm{~Hz}), 135.0(\mathrm{~d}, J=2.8 \mathrm{~Hz}), 132.7(\mathrm{~d}, J=1.7 \mathrm{~Hz}), 131.3(\mathrm{~d}, J=6.6 \mathrm{~Hz}), 131.2$, $130.5,129.9(\mathrm{~d}, J=1.8 \mathrm{~Hz}), 129.5,127.7$ (d, $J=11.4 \mathrm{~Hz}), 127.3,126.9,126.7,126.4,126.0,125.1,33.5$ (d, $J=23.5 \mathrm{~Hz}$ ), 32.0 (d, $J=25.3 \mathrm{~Hz}$ ), 31.9 (d, $J=15.5 \mathrm{~Hz}$ ), 30.3 (d, $J=14.2 \mathrm{~Hz})$, .

${ }^{31} \mathrm{P}\left\{{ }^{1} \mathrm{H}\right\}$ NMR $\left(\mathrm{CDCl}_{3}, 162 \mathrm{MHz}\right): \delta 21.9$ (s).

HRMS (ESI) m/z: [M+H] ${ }^{+}$Calcd for $\mathrm{C}_{34} \mathrm{H}_{38} \mathrm{P}$ 477.2705; Found 477.2710. 
(E)-Dicyclohexyl(2'-(1,2-diphenylvinyl)-6'-methyl-[1,1'-biphenyl]-2-yl)phosphane (3ea). Following<smiles>Cc1cccc(C(=Cc2ccccc2)c2ccccc2)c1-c1ccccc1</smiles>
the general procedure A using 2-(dicyclohexylphosphino)-2'-methyl-biphenyl (MePhos) (72.8 mg, $0.2 \mathrm{mmol}$ ) and diphenylacetylene $(36.1 \mathrm{mg}, 0.2 \mathrm{mmol})$, the residue was purified by flash chromatography on silica gel (heptane- $\mathrm{Et}_{2} \mathrm{O}=99: 1$ ) to afford 3 ea $(73.8 \mathrm{mg}, 68 \%)$ as pale yellow oil.

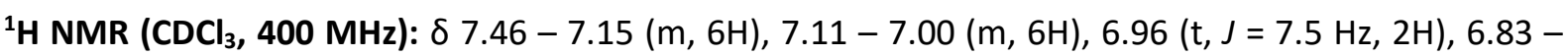
$6.59(\mathrm{~m}, 3 \mathrm{H}), 6.32(\mathrm{dd}, J=7.9,3.8 \mathrm{~Hz}, 1 \mathrm{H}), 1.97(\mathrm{~s}, 3 \mathrm{H}), 1.88-1.43(\mathrm{~m}, 11 \mathrm{H}), 1.36-0.95(\mathrm{~m}, 11 \mathrm{H})$.

${ }^{13} \mathrm{C}\left\{{ }^{1} \mathrm{H}\right\}$ NMR $\left(\mathrm{CDCl}_{3}, 101 \mathrm{MHz}\right): \delta 146.8(\mathrm{~d}, J=31.6 \mathrm{~Hz}), 144.5,143.8,141.3,137.4,136.6,132.1$, $131.0,129.5,129.5,129.2,129.2,128.7,128.5$ (d, J = 2.9 Hz), 128.3 , $127.7,127.6,127.2,126.4$ (d, J = 7.1 Hz), $125.5(\mathrm{~d}, J=3.6 \mathrm{~Hz}), 35.9(\mathrm{~d}, J=8.8 \mathrm{~Hz}), 32.4(\mathrm{~d}, J=8.5 \mathrm{~Hz}), 31.9,31.0,30.3,30.2(\mathrm{~d}, J$ $=2.9 \mathrm{~Hz}), 29.1,28.9$ (d, $J=6.2 \mathrm{~Hz}), 27.8(\mathrm{~d}, J=7.3 \mathrm{~Hz}), 27.1$, 26.4, $22.7,21.2$.

${ }^{31} \mathrm{P}\left\{{ }^{1} \mathrm{H}\right\}$ NMR $\left(\mathrm{CDCl}_{3}, 162 \mathrm{MHz}\right): \delta-7.8(\mathrm{~s})$.

HRMS (ESI) m/z: [M+H] $]^{+}$Calcd for $\mathrm{C}_{39} \mathrm{H}_{44} \mathrm{P}$ 543.3175; Found 543.3176.<smiles>CN(C)c1cccc(C(=Cc2ccccc2)c2ccccc2)c1-c1ccccc1</smiles>

(E)-2'-(Dicyclohexylphosphanyl)-6-(1,2-diphenylvinyl)-N,N-dimethyl-[1,1'biphenyl]-2-amine (3fa). Following the general procedure $\mathbf{A}$ using 2(dicyclohexylphosphino)-2'-dimethylamimo-biphenyl (DavePhos) (78.8 mg, 0.2 $\mathrm{mmol}$ ) and diphenylacetylene $(36.1 \mathrm{mg}, 0.2 \mathrm{mmol})$, the residue was purified by flash chromatography on silica gel (heptane- $\mathrm{Et}_{2} \mathrm{O}=99: 1$ ) to afford $\mathbf{3 f a}(91.4 \mathrm{mg}, 80 \%)$ as a pale yellow solid; $\mathrm{mp}=86-88^{\circ} \mathrm{C}$.

${ }^{1}{ }^{H}$ NMR $\left(\mathrm{CDCl}_{3}, 400 \mathrm{MHz}\right): \delta 7.49(\mathrm{~d}, J=6.9 \mathrm{~Hz}, 1 \mathrm{H}), 7.42-7.35(\mathrm{~m}, 1 \mathrm{H}), 7.15-7.04(\mathrm{~m}, 9 \mathrm{H}), 6.92-$ $6.81(\mathrm{~m}, 5 \mathrm{H}), 6.72-6.65(\mathrm{~m}, 1 \mathrm{H}), 6.47(\mathrm{~s}, 1 \mathrm{H}), 2.51(\mathrm{~s}, 6 \mathrm{H}), 2.08-1.61(\mathrm{~m}, 11 \mathrm{H}), 1.36-1.16(\mathrm{~m}, 11 \mathrm{H})$. ${ }^{13} \mathrm{C}\left\{{ }^{1} \mathrm{H}\right\}$ NMR (CDCl $\left.3,101 \mathrm{MHz}\right): \delta 152.9,145.8(\mathrm{~d}, J=31.3 \mathrm{~Hz}), 144.9,143.2,141.3,137.7,136.9$ (d, $J$ $=16.8 \mathrm{~Hz}$ ), 136.3 , $132.7,131.9$, $131.4,130.0$, 129.2 , 127.9 , 127.7 , $127.6,126.6$ (d, J = 8.3 Hz), 126.2 , 125.5 , 117.7 , 44.9 , 35.9 (d, $J=16.1 \mathrm{~Hz}), 33.4$ (d, $J=14.5 \mathrm{~Hz}$ ), 32.7 (d, $J=21.5 \mathrm{~Hz}$ ), $31.9,30.8$ (d, $J=$ $14.6 \mathrm{~Hz}$ ), $30.6(\mathrm{~d}, J=18.0 \mathrm{~Hz}$ ), $29.0,28.3(\mathrm{~d}, J=14.4 \mathrm{~Hz}), 28.0(\mathrm{~d}, J=4.2 \mathrm{~Hz}), 27.9,27.2(\mathrm{~d}, J=10.8 \mathrm{~Hz})$, $26.6(\mathrm{~d}, J=7.1 \mathrm{~Hz})$.

${ }^{31} \mathrm{P}\left\{{ }^{1} \mathrm{H}\right\} \mathrm{NMR}\left(\mathrm{CDCl}_{3}, 162 \mathrm{MHz}\right): \delta-8.2(\mathrm{~s})$.

HRMS (ESI) m/z: [M+H] $]^{+}$Calcd for $\mathrm{C}_{40} \mathrm{H}_{47} \mathrm{NP}$ 572.3440; Found 572.3441 . 
<smiles>CN(C)c1ccc(-c2ccccc2P(=O)(O)c2ccccc2)c(/C(=C/c2ccccc2)c2ccccc2)c1</smiles>
biphenyl]-4-amine (3ga). Following the general procedure A using 2(dicyclohexylphosphino)-4'-dimethylamimo-biphenyl (78.8 mg, $0.2 \mathrm{mmol}$ ) and diphenylacetylene $(36.1 \mathrm{mg}, 0.2 \mathrm{mmol})$, the residue was purified by flash chromatography on silica gel (heptane- $\left.\mathrm{Et}_{2} \mathrm{O}=99: 1\right)$ to afford $3 g a(90.3 \mathrm{mg}, 79 \%)$ as a pale yellow solid; $\mathrm{mp}=101-103^{\circ} \mathrm{C}$.

${ }^{1} \mathrm{H}$ NMR $\left(\mathrm{CDCl}_{3}, 400 \mathrm{MHz}\right): \delta 7.36(\mathrm{~d}, J=7.6 \mathrm{~Hz}, 1 \mathrm{H}), 7.11-6.97(\mathrm{~m}, 10 \mathrm{H}), 6.88(\mathrm{dd}, J=19.8,7.5 \mathrm{~Hz}$, $3 \mathrm{H}), 6.76(\mathrm{~s}, 2 \mathrm{H}), 6.68(\mathrm{dd}, J=8.0,3.9 \mathrm{~Hz}, 1 \mathrm{H}), 6.65(\mathrm{~s}, 1 \mathrm{H}), 3.02(\mathrm{~s}, 6 \mathrm{H}), 1.91-1.52(\mathrm{~m}, 10 \mathrm{H}), 1.47-$ $0.97(\mathrm{~m}, 12 \mathrm{H})$.

${ }^{13} \mathrm{C}\left\{{ }^{1} \mathrm{H}\right\}$ NMR $\left(\mathrm{CDCl}_{3}, 101 \mathrm{MHz}\right): \delta 149.7,149.3(\mathrm{~d}, J=14.5 \mathrm{~Hz}), 144.4,143.76,141.0,137.6,134.9$, $134.7,132.8$, $132.2,131.3$ (d, J = 5.6 Hz), 131.0 , $129.9,129.4,127.6$ (d, J = $4.3 \mathrm{~Hz}), 127.2,126.4$ (d, $J=11.1 \mathrm{~Hz}), 125.3$, 114.6 , 110.2 , $40.6,36.6$ (d, $J=16.2 \mathrm{~Hz}), 32.9$ (d, $J=12.8 \mathrm{~Hz}), 31.0(\mathrm{~d}, J=13.9 \mathrm{~Hz})$, $30.5(\mathrm{~d}, J=19.6 \mathrm{~Hz}), 30.1(\mathrm{~d}, J=13.4 \mathrm{~Hz}), 29.0(\mathrm{~d}, J=5.1 \mathrm{~Hz}), 27.8,27.8(\mathrm{~d}, J=3.6 \mathrm{~Hz}), 27.3,27.1(\mathrm{~d}, J$ $=5.2 \mathrm{~Hz}), 27.0$, $26.5(\mathrm{~d}, J=10.6 \mathrm{~Hz})$.

${ }^{31} \mathrm{P}\left\{{ }^{1} \mathrm{H}\right\}$ NMR $\left(\mathrm{CDCl}_{3}, 162 \mathrm{MHz}\right): \delta-10.7$ (s) [presence of $\mathbf{1 g}$ phosphine oxide at $45.4 \mathrm{ppm}(<5 \%)$ ].

HRMS (ESI) m/z: [M+H] ${ }^{+}$Calcd for $\mathrm{C}_{40} \mathrm{H}_{47} \mathrm{NP}$ 572.3440; Found 572.3433.<smiles>Pc1cccn1-c1ccccc1C(=Cc1ccccc1)c1ccccc1</smiles>
(E)-2-(Diphenylphosphanyl)-1-(2-(1,2-diphenylvinyl)phenyl)-1H-pyrrole (3ha). Following the general procedure A using N-phenylpyrrol-2-yldiphenylphosphine (65.6 $\mathrm{mg}, 0.2 \mathrm{mmol}$ ) and diphenylacetylene $(36.1 \mathrm{mg}, 0.2 \mathrm{mmol})$, the residue was purified by flash chromatography on silica gel (heptane- $\mathrm{Et}_{2} \mathrm{O}=99: 1$ ) to afford 3ha (77.8 $\mathrm{mg}, 77 \%$ ) as a white solid; $\mathrm{mp}=82-84^{\circ} \mathrm{C}$.

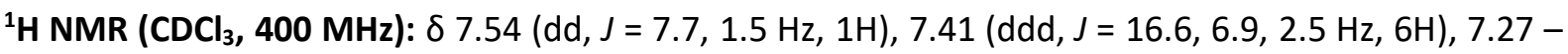
$7.13(\mathrm{~m}, 11 \mathrm{H}), 7.11-7.01(\mathrm{~m}, 5 \mathrm{H}), 7.00-6.93(\mathrm{~m}, 1 \mathrm{H}), 6.84(\mathrm{td}, J=2.9,1.6 \mathrm{~Hz}, 1 \mathrm{H}), 6.76(\mathrm{~s}, 1 \mathrm{H}), 6.19$ $-6.14(\mathrm{~m}, 1 \mathrm{H}), 6.02(\mathrm{dd}, J=3.6,1.6 \mathrm{~Hz}, 1 \mathrm{H})$.

${ }^{13} \mathrm{C}\left\{{ }^{1} \mathrm{H}\right\} \operatorname{NMR}\left(\mathrm{CDCl}_{3}, 101 \mathrm{MHz}\right): \delta 142.1,140.4,139.5,138.6(\mathrm{~d}, J=2.3 \mathrm{~Hz}), 138.1,137.2,133.3(\mathrm{~d}, J$ $=19.5 \mathrm{~Hz}), 131.4,131.3(\mathrm{~d}, J=2.1 \mathrm{~Hz}), 129.7(\mathrm{~d}, J=6.1 \mathrm{~Hz}), 129.5(\mathrm{~d}, J=2.0 \mathrm{~Hz}), 129.4(\mathrm{~d}, J=3.9 \mathrm{~Hz})$, $129.1(\mathrm{~d}, J=2.5 \mathrm{~Hz}), 128.2,128.0$ (d, J = $11.3 \mathrm{~Hz}), 127.7,127.2$ (d, $J=2.5 \mathrm{~Hz}), 127.2,126.9,118.9$, 109.6

${ }^{31} \mathrm{P}\left\{{ }^{1} \mathrm{H}\right\}$ NMR $\left(\mathrm{CDCl}_{3}, 162 \mathrm{MHz}\right): \delta-30.7$ (s).

HRMS (ESI) m/z: [M+H] ${ }^{+}$Calcd for $\mathrm{C}_{36} \mathrm{H}_{29} \mathrm{NP}$ 506.2032; Found 506.2036. 
<smiles>Cn1c(-c2ccccc2P(=O)(F)c2ccccc2)c(/C(=C/c2ccccc2)c2ccccc2)c2ccccc21</smiles>

(E)-2-(2-(Dicyclohexylphosphanyl)phenyl)-3-(1,2-diphenylvinyl)-1-methyl1H-indole (3ia). Following the general procedure A using 2-[2(dicyclohexylphosphino)phenyl]-1-methyl-1H-indole (CM-Phos) $(80.8 \mathrm{mg}$, $0.2 \mathrm{mmol})$ and diphenylacetylene $(36.1 \mathrm{mg}, 0.2 \mathrm{mmol})$, the residue was purified by flash chromatography on silica gel (heptane- $\mathrm{Et}_{2} \mathrm{O}=99: 1$ ) to afford 3ia (46.5

$\mathrm{mg}, 40 \%$ ) as pale yellow oil.

${ }^{1} \mathrm{H} \mathrm{NMR}\left(\mathrm{CDCl}_{3}, 400 \mathrm{MHz}\right): \delta 7.36(\mathrm{dd}, J=8.0,4.4 \mathrm{~Hz}, 2 \mathrm{H}), 7.28-7.19(\mathrm{~m}, 5 \mathrm{H}), 7.19-7.10(\mathrm{~m}, 4 \mathrm{H}), 7.08$ $-7.00(\mathrm{~m}, 5 \mathrm{H}), 6.96-6.90(\mathrm{~m}, 2 \mathrm{H}), 6.60(\mathrm{~s}, 1 \mathrm{H}), 3.46(\mathrm{~s}, 3 \mathrm{H}), 1.90-1.55(\mathrm{~m}, 11 \mathrm{H}), 1.35-1.04(\mathrm{~m}, 11 \mathrm{H})$. ${ }^{13} \mathrm{C}\left\{{ }^{1} \mathrm{H}\right\}$ NMR $\left(\mathrm{CDCl}_{3}, 101 \mathrm{MHz}\right): \delta 141.7,137.9,136.5(\mathrm{~d}, J=4.9 \mathrm{~Hz}), 136.4,132.7,132.7(\mathrm{~d}, J=5.7 \mathrm{~Hz})$, $130.6(d, J=2.1 \mathrm{~Hz}), 130.4(\mathrm{~d}, J=10.0 \mathrm{~Hz}), 129.2,128.6,127.9,127.7(\mathrm{~d}, J=4.5 \mathrm{~Hz}), 127.6,127.5$, $127.4,126.9,125.7$ (d, $J=5.5 \mathrm{~Hz}), 121.4$ (d, $J=7.2 \mathrm{~Hz}), 120.5,119.6,117.9,109.3,35.3$ (d, $J=14.9$ $\mathrm{Hz}$ ), $34.9(\mathrm{~d}, J=15.1 \mathrm{~Hz}), 31.0(\mathrm{~d}, J=3.1 \mathrm{~Hz}$ ), 30.7 (d, J=6.9 Hz), 30.5, 30.1 (d, J=13.3 Hz), 29.9 , 29.7, $27.5(\mathrm{~d}, J=10.0 \mathrm{~Hz}$ ), $27.3(\mathrm{~d}, J=11.0 \mathrm{~Hz}), 27.2(\mathrm{~d}, J=10.0 \mathrm{~Hz}), 26.7$ (d, J = 6.1 Hz), $26.4(\mathrm{~d}, J=7.8 \mathrm{~Hz})$. ${ }^{31} \mathrm{P}\left\{{ }^{1} \mathrm{H}\right\}$ NMR $\left(\mathrm{CDCl}_{3}, 162 \mathrm{MHz}\right): \delta-9.81(\mathrm{~s})$.

HRMS (ESI) m/z: [M+H] ${ }^{+}$Calcd for $\mathrm{C}_{41} \mathrm{H}_{45} \mathrm{NP}$ 582.3284; Found 582.3287 .<smiles>CNc1cccc(C(=Cc2ccc(Br)cc2)c2ccc(Br)cc2)c1-c1ccccc1[PH](C)(=O)=O</smiles>

(E)-6-(1,2-Bis(4-bromophenyl)vinyl)-2'-(dicyclohexylphosphanyl)-N,Ndimethyl-[1,1'-biphenyl]-2-amine (3fb). Following the general procedure A using 2-(dicyclohexylphosphino)-2'-dimethylamimo-biphenyl (DavePhos) (78.8 mg, $0.2 \mathrm{mmol}$ ), and Bis(4-bromophenyl)acetylene (67.2 $\mathrm{mg}, 0.2 \mathrm{mmol}$ ), the residue was purified by flash chromatography on silica gel (heptane-Et $2 \mathrm{O}=99: 1)$ to afford $\mathbf{3} \mathrm{fb}(111.9 \mathrm{mg}, 77 \%)$ as a pale yellow solid; $\mathrm{mp}=111-113^{\circ} \mathrm{C}$.

${ }^{1} \mathrm{H}$ NMR $\left(\mathrm{CDCl}_{3}, 400 \mathrm{MHz}\right): \delta 7.45(\mathrm{~d}, J=7.1 \mathrm{~Hz}, 1 \mathrm{H}), 7.36(\mathrm{t}, J=8.0 \mathrm{~Hz}, 1 \mathrm{H}), 7.22-7.02(\mathrm{~m}, 8 \mathrm{H}), 6.90$ $(\mathrm{d}, J=8.3 \mathrm{~Hz}, 1 \mathrm{H}), 6.73(\mathrm{~d}, J=8.2 \mathrm{~Hz}, 2 \mathrm{H}), 6.63(\mathrm{~d}, J=8.1 \mathrm{~Hz}, 2 \mathrm{H}), 6.40(\mathrm{~s}, 1 \mathrm{H}), 2.50(\mathrm{~s}, 6 \mathrm{H}), 1.99-1.65$ (m, 11H), $1.36-1.10(\mathrm{~m}, 11 \mathrm{H})$.

${ }^{13} \mathrm{C}\left\{{ }^{1} \mathrm{H}\right\}$ NMR $\left(\mathrm{CDCl}_{3}, 101 \mathrm{MHz}\right): \delta 153.1,145.5(\mathrm{~d}, J=31.0 \mathrm{~Hz}), 144.1,142.8,139.9,136.9(\mathrm{~d}, J=17.8$ Hz), $136.2,132.6,131.9,131.5,131.0$ (d, $J=15.4 \mathrm{~Hz}), 130.7,130.6,128.1,126.7,125.5$ (d, $J=48.0$ Hz), 120.6 (d, $J=51.8 \mathrm{~Hz}$ ), 118.2 , $45.0,35.9$ (d, $J=16.3 \mathrm{~Hz}$ ), $33.1,32.9$ (d, $J=14.1 \mathrm{~Hz}), 32.6,31.9$, 30.9 (d, J = 14.2 Hz), 30.5 (d, J = 18.2 Hz), 28.3 (d, J = 14.9 Hz), $27.8,27.7$ (d, J = 3.4 Hz), 27.05(d, J = $10.2 \mathrm{~Hz}), 26.5$.

${ }^{31} \mathrm{P}\left\{{ }^{1} \mathrm{H}\right\}$ NMR $\left(\mathrm{CDCl}_{3}, 162 \mathrm{MHz}\right): \delta 8.0$ (s) [presence of $1 \mathrm{f}$ phosphine oxide at $\left.44.5 \mathrm{ppm}(<5 \%)\right]$. HRMS (ESI) m/z: [M+H] $]^{+}$Calcd for $\mathrm{C}_{40} \mathrm{H}_{45} \mathrm{Br}_{2} \mathrm{NP}$ 728.1651; Found 728.1653. 
(E)-(2'-(1,2-Bis(4-bromophenyl)vinyl)-[1,1'-biphenyl]-2-<smiles>O=Pc1ccccc1-c1ccccc1C(=Cc1ccc(Br)cc1)c1ccc(Br)cc1</smilesyl)dicyclohexylphosphane (3ab). Following the general procedure $\mathbf{A}$ using 2-(dicyclohexylphosphino)biphenyl (70.1 mg, $0.2 \mathrm{mmol}$ ), and Bis(4bromophenyl)acetylene $(67.2 \mathrm{mg}, 0.2 \mathrm{mmol})$, the residue was purified by flash chromatography on silica gel (heptane-Et ${ }_{2} \mathrm{O}=99: 1$ ) to afford 3ab (111.1 $\mathrm{mg}, 81 \%$ ) as white solid; $\mathrm{mp}=179-181^{\circ} \mathrm{C}$.

${ }^{1} \mathrm{H}$ NMR $\left(\mathrm{CDCl}_{3}, 400 \mathrm{MHz}\right) \delta: 7.42-7.30(\mathrm{~m}, 5 \mathrm{H}), 7.23-7.12(\mathrm{~m}, 6 \mathrm{H})$, $6.99(\mathrm{t}, J=7.6 \mathrm{~Hz}, 1 \mathrm{H}), 6.82(\mathrm{~d}, J=8.1 \mathrm{~Hz}, 2 \mathrm{H}), 6.68(\mathrm{dd}, J=7.4,2.4 \mathrm{~Hz}$, $2 \mathrm{H}), 6.56(\mathrm{~s}, 1 \mathrm{H}), 1.85-1.43(\mathrm{~m}, 11 \mathrm{H}), 1.32-0.90(\mathrm{~m}, 11 \mathrm{H})$.

${ }^{13} \mathrm{C}\left\{{ }^{1} \mathrm{H}\right\}$ NMR $\left(\mathrm{CDCl}_{3}, 101 \mathrm{MHz}\right): \delta 148.9(\mathrm{~d}, J=29.7 \mathrm{~Hz}), 143.1(\mathrm{~d}, J=2.0 \mathrm{~Hz}), 142.4,139.6,135.9,132.1$ (d, $J=3.1 \mathrm{~Hz}$ ), $131.5(\mathrm{~d}, J=1.8 \mathrm{~Hz}), 131.1,130.9$ (d, $J=8.0 \mathrm{~Hz}$ ), $130.6(\mathrm{~d}, J=15.0 \mathrm{~Hz}), 130.2,127.4$, $126.8,126.1,120.9,120.6,36.4,32.3,30.8$ (d, $J=5.3 \mathrm{~Hz}), 30.7,30.4,30.3,28.9,27.7$ (d, $J=10.0$ $\mathrm{Hz}), 27.1,26.9(\mathrm{~d}, J=9.1 \mathrm{~Hz}), 26.5,26.3$.

${ }^{31} \mathrm{P}\left\{{ }^{1} \mathrm{H}\right\}$ NMR $\left(\mathrm{CDCl}_{3}, 162 \mathrm{MHz}\right): \delta-10.5$ (s) [presence of 1a phosphine at $-13.4 \mathrm{ppm}(<5 \%)$ ].

HRMS (ESI) m/z: [M+H] $]^{+}$Calcd for $\mathrm{C}_{38} \mathrm{H}_{40} \mathrm{Br}_{2} \mathrm{P}$ 685.1228; Found 685.1228.<smiles>Cc1ccc(/C=C(\c2ccc(C)cc2)c2ccccc2-c2ccccc2OC(F)(F)F)cc1</smiles>

(E)-Dicyclohexyl(2'-(1,2-di-p-tolylvinyl)-[1,1'-biphenyl]-2-yl)phosphane (3ac). Following the general procedure $A$ using 2(dicyclohexylphosphino)biphenyl (70.1 mg, $0.2 \mathrm{mmol}$ ), and 1,2-di-ptolylethyne $(41.5 \mathrm{mg}, 0.2 \mathrm{mmol})$, the residue was purified by flash chromatography on silica gel (heptane-Et $2 \mathrm{O}=99: 1$ ) to afford 3ac (91.3mg, 82\%) as white solid; $\mathrm{mp}=103-105^{\circ} \mathrm{C}$.

${ }^{1} \mathrm{H}$ NMR $\left(\mathrm{CDCl}_{3}, 400 \mathrm{MHz}\right): \delta 7.44-7.30(\mathrm{~m}, 4 \mathrm{H}), 7.15(\mathrm{t}, J=6.2 \mathrm{~Hz}, 2 \mathrm{H}), 6.99(\mathrm{t}, J=7.6 \mathrm{~Hz}, 1 \mathrm{H}), 6.93-$ $6.84(\mathrm{~m}, 6 \mathrm{H}), 6.80(\mathrm{dd}, \mathrm{J}=13.1,6.2 \mathrm{~Hz}, 3 \mathrm{H}), 6.52(\mathrm{~s}, 1 \mathrm{H}), 2.33(\mathrm{~s}, 3 \mathrm{H}), 2.25(\mathrm{~s}, 3 \mathrm{H}), 1.90-1.47(\mathrm{~m}, 11 \mathrm{H})$, $1.40-0.96(\mathrm{~m}, 11 \mathrm{H})$.

${ }^{13} \mathrm{C}\left\{{ }^{1} \mathrm{H}\right\}$ NMR $\left(\mathrm{CDCl}_{3}, 101 \mathrm{MHz}\right): \delta 149.5(\mathrm{~d}, J=30.5 \mathrm{~Hz}), 144.1(\mathrm{~d}, J=1.9 \mathrm{~Hz}), 141.7(\mathrm{~d}, J=35.2 \mathrm{~Hz}), 138.3$ , $136.0(\mathrm{~d}, J=13.8 \mathrm{~Hz}), 134.7,134.3(\mathrm{~d}, J=20.0 \mathrm{~Hz}), 132.1(\mathrm{~d}, J=16.9 \mathrm{~Hz}), 131.3,130.6,130.3,129.9$ , $129.2,128.4$ (d, J = 23.2 Hz), 127.2 (d, J = 29.5 Hz), 125.8 (d, J = 27.6 Hz), 36.6 (d, J = $16.4 \mathrm{~Hz}$ ), 32.9 (d, $J=12.8 \mathrm{~Hz}$ ), 30.9 (d, $J=13.6 \mathrm{~Hz}), 30.5,30.4,30.2$ (d, $J=13.2 \mathrm{~Hz}), 29.1,27.7,27.2,27.1$ (d, $J=11.5$ $\mathrm{Hz}), 26.6,26.4,21.3,21.2$.

${ }^{31} \mathrm{P}\left\{{ }^{1} \mathrm{H}\right\}$ NMR $\left(\mathrm{CDCl}_{3}, 162 \mathrm{MHz}\right): \delta-10.9(\mathrm{~s})$.

HRMS (ESI) m/z: [M+H] $]^{+}$Calcd for $\mathrm{C}_{40} \mathrm{H}_{46} \mathrm{P}$ 557.3331; Found 557.3328. 
<smiles>COc1ccc(C=C(c2ccc(OC)cc2)c2ccccc2-c2ccccc2P(C)(=O)c2ccccc2)cc1</smiles>

(E)-(2'-(1,2-Bis(4-methoxyphenyl)vinyl)-[1,1'-biphenyl]-2yl)dicyclohexylphosphane (3ad). Following the general procedure A using 2-(dicyclohexylphosphino)biphenyl (70.1 mg, $0.2 \mathrm{mmol}$ ), and 1,2bis(3-methoxyphenyl)ethyne $(47.8 \mathrm{mg}, 0.2 \mathrm{mmol})$, the residue was purified by flash chromatography on silica gel (heptane-Et ${ }_{2} \mathrm{O}=99: 1$ ) to afford 3ad (100.1 mg, $85 \%)$ as a white solid; $\mathrm{mp}=164-166^{\circ} \mathrm{C}$.

${ }^{1} \mathrm{H}$ NMR $\left(\mathrm{CDCl}_{3}, 400 \mathrm{MHz}\right): \delta 7.48-7.30(\mathrm{~m}, 4 \mathrm{H}), 7.16(\mathrm{~d}, J=7.4 \mathrm{~Hz}, 2 \mathrm{H}), 7.03(\mathrm{t}, J=7.5 \mathrm{~Hz}, 1 \mathrm{H}), 6.95$ (d, J = 8.4 Hz, 2H), $6.85(\mathrm{~d}, J=8.2 \mathrm{~Hz}, 2 \mathrm{H}), 6.82-6.76(\mathrm{~m}, 1 \mathrm{H}), 6.65(\mathrm{~d}, J=8.3 \mathrm{~Hz}, 4 \mathrm{H}), 6.52(\mathrm{~s}, 1 \mathrm{H}), 3.81$ $(\mathrm{s}, 3 \mathrm{H}), 3.75(\mathrm{~s}, 3 \mathrm{H}), 1.93-1.49(\mathrm{~m}, 11 \mathrm{H}), 1.44-0.97(\mathrm{~m}, 11 \mathrm{H})$.

${ }^{13} \mathrm{C}\left\{{ }^{1} \mathrm{H}\right\}$ NMR $\left(\mathrm{CDCl}_{3}, 101 \mathrm{MHz}\right): \delta 158.2(\mathrm{~d}, J=28.4 \mathrm{~Hz}), 149.6(\mathrm{~d}, J=30.8 \mathrm{~Hz}), 144.4(\mathrm{~d}, J=2.0 \mathrm{~Hz}), 141.8$ (d, $J=5.6 \mathrm{~Hz}$ ), 140.3 , 134.3, 134.1 , 133.9, 132.2 (d, $J=3.3 \mathrm{~Hz}), 132.0$ (d, $J=2.4 \mathrm{~Hz}$ ), 131.2 (d, $J=2.0$ Hz), 130.7 , 130.6 , 130.5 , 130.4 , 130.2 , 127.2 (d, J = 23.5 Hz), 125.9 (d, J = 23.2 Hz), 113.2 (d, J = 23.5 Hz) , 55.2 , 55.1 , $36.7(\mathrm{~d}, J=16.5 \mathrm{~Hz}), 32.8(\mathrm{~d}, J=12.7 \mathrm{~Hz}), 31.0(\mathrm{~d}, J=14.0 \mathrm{~Hz}), 30.5(\mathrm{~d}, J=19.9 \mathrm{~Hz})$, $30.2(\mathrm{~d}, J=13.0 \mathrm{~Hz}), 29.1(\mathrm{~d}, J=4.8 \mathrm{~Hz}), 27.8,27.7(\mathrm{~d}, J=5.3 \mathrm{~Hz}), 27.3$, 27.1 , $27.0,26.5$ (d, J = 15.1 $\mathrm{Hz}$ ).

${ }^{31} \mathrm{P}\left\{{ }^{1} \mathrm{H}\right\}$ NMR $\left(\mathrm{CDCl}_{3}, 162 \mathrm{MHz}\right): \delta-10.9(\mathrm{~s})$

HRMS (ESI) m/z: [M+H] ${ }^{+}$Calcd for $\mathrm{C}_{40} \mathrm{H}_{46} \mathrm{O}_{2} \mathrm{P}$ 589.3230; Found 589.3231.<smiles>O=C(O)c1ccccc1-c1ccccc1C(=Cc1ccc(C(F)(F)F)cc1)c1ccc(C(F)(F)F)cc1</smiles>

(E)-(2'-(1,2-Bis(4-(trifluoromethyl)phenyl)vinyl)-[1,1'-biphenyl]-2yl)dicyclohexylphosphane (3ae). Following the general procedure A using 2-(dicyclohexylphosphino)biphenyl (70.1 mg, $0.2 \mathrm{mmol}$ ), and 1,2bis(4-(trifluoromethyl)phenyl)ethyne $(63.2 \mathrm{mg}, 0.2 \mathrm{mmol})$, the residue was purified by flash chromatography on silica gel (heptane- $\mathrm{Et}_{2} \mathrm{O}=99: 1$ ) to afford 3ae (110.3 $\mathrm{mg}, 83 \%)$ as a white solid; $\mathrm{mp}=124-126^{\circ} \mathrm{C}$.

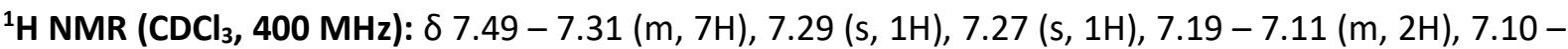
$7.02(\mathrm{~m}, 2 \mathrm{H}), 6.88(\mathrm{t}, J=6.3 \mathrm{~Hz}, 3 \mathrm{H}), 6.54(\mathrm{ddd}, J=7.7,3.9,1.3 \mathrm{~Hz}, 1 \mathrm{H}), 1.88-1.53(\mathrm{~m}, 11 \mathrm{H}), 1.35-$ $0.88(\mathrm{~m}, 11 \mathrm{H})$.

${ }^{13} \mathrm{C}\left\{{ }^{1} \mathrm{H}\right\}$ NMR (CDCl $\left.3,101 \mathrm{MHz}\right): \delta 148.6(\mathrm{~d}, J=28.4 \mathrm{~Hz}), 144.2,143.8,142.8(\mathrm{~d}, J=1.9 \mathrm{~Hz}), 140.2,132.1$ (d, $J=2.6 \mathrm{~Hz}$ ), 131.1, 130.4, 129.9, 129.5, 129.1, 128.9, 128.8 (d, J = 3.8 Hz), 128.6 (d, J = 3.7 Hz), 127.9, 127.2, 126.2, 125.2 (d, $J=11.3 \mathrm{~Hz}), 124.8$ (q, $J=271.1 \mathrm{~Hz}), 124.7$ (q, $J=271.5 \mathrm{~Hz}), 123.0$ (d, $J=11.1 \mathrm{~Hz}$ ), $120.8(\mathrm{~d}, J=11.1 \mathrm{~Hz}), 36.3(\mathrm{~d}, J=17.6 \mathrm{~Hz}), 31.9(\mathrm{~d}, J=9.6 \mathrm{~Hz}), 30.6,30.1,28.8,28.7,27.5,27.0,26.9$, 26.8, $26.4(\mathrm{~d}, J=5.1 \mathrm{~Hz}), 26.2$.

${ }^{31} \mathrm{P}\left\{{ }^{1} \mathrm{H}\right\} \mathrm{NMR}\left(\mathrm{CDCl}_{3}, 162 \mathrm{MHz}\right): \delta-9.6(\mathrm{~s})$.

${ }^{19} \mathrm{~F}\left\{{ }^{1} \mathrm{H}\right\} \mathrm{NMR}\left(\mathrm{CDCl}_{3}, 282 \mathrm{MHz}\right): \delta-62.5(\mathrm{~s}),-62.6(\mathrm{~s})$.

HRMS (ESI) m/z: [M+H] $]^{+}$Calcd for $\mathrm{C}_{40} \mathrm{H}_{40} \mathrm{~F}_{6} \mathrm{P}$ 665.2766; Found 665.2772. 
<smiles>C/C=C(\CC)c1ccccc1-n1cccc1-c1ccccc1</smiles>

(E)-2-(Diphenylphosphanyl)-1-(2-(hex-3-en-3-yl)phenyl)-1H-pyrrole

(3hf).

Following the general procedure $\mathbf{A}$ using $\mathrm{N}$-phenylpyrrol-2-yldiphenylphosphine (65.6 $\mathrm{mg}, 0.2 \mathrm{mmol})$, and 3-hexyne $(23 \mu \mathrm{L}, 0.2 \mathrm{mmol})$, the residue was purified by flash chromatography on silica gel (heptane-Et ${ }_{2} \mathrm{O}=99: 1$ ) to afford $\mathbf{3 h f}(55.7 \mathrm{mg}$, $68 \%)$ as a colorless oil.

${ }^{1}{ }^{H}$ NMR $\left(\mathrm{CDCl}_{3}, 400 \mathrm{MHz}\right): \delta 7.36-7.29(\mathrm{~m}, 11 \mathrm{H}), 7.21-7.10(\mathrm{~m}, 2 \mathrm{H}), 6.95-6.91(\mathrm{~m}, 2 \mathrm{H}), 6.33-6.27$ $(\mathrm{m}, 1 \mathrm{H}), 6.18(\mathrm{dd}, J=3.6,1.7 \mathrm{~Hz}, 1 \mathrm{H}), 5.43(\mathrm{t}, J=7.2 \mathrm{~Hz}, 1 \mathrm{H}), 2.21-1.84(\mathrm{~m}, 4 \mathrm{H}), 0.96(\mathrm{t}, J=7.5 \mathrm{~Hz}, 3 \mathrm{H})$, $0.81(\mathrm{t}, J=7.6 \mathrm{~Hz}, 3 \mathrm{H})$.

${ }^{13} \mathrm{C}\left\{{ }^{1} \mathrm{H}\right\}$ NMR (CDCl, $\left.101 \mathrm{MHz}\right): \delta$ 141.4, 140.5, $138.2(\mathrm{~d}, J=7.0 \mathrm{~Hz}), 137.7(\mathrm{~d}, J=2.7 \mathrm{~Hz}), 133.3(\mathrm{~d}, J=$ 19.7 Hz), 132.6, 131.0, 129.1 (d, $J=2.0 \mathrm{~Hz}$ ), 128.6 (d, $J=5.2 \mathrm{~Hz}$ ), 128.2 (d, $J=6.4 \mathrm{~Hz}$ ), 127.9, 127.7 (d, $J$ $=2.5 \mathrm{~Hz}$ ), 126.6, 119.1 (d, $J=2.6 \mathrm{~Hz}), 109.3(\mathrm{~d}, J=1.4 \mathrm{~Hz}), 22.1,21.3,14.2,13.6$ (d, $J=2.3 \mathrm{~Hz})$.

${ }^{31} \mathrm{P}\left\{{ }^{1} \mathrm{H}\right\}$ NMR $\left(\mathrm{CDCl}_{3}, 162 \mathrm{MHz}\right): \delta-33.2$ (s).

HRMS (ESI) m/z: [M+H] $]^{+}$Calcd for $\mathrm{C}_{28} \mathrm{H}_{29} \mathrm{NP}$ 410.2032; Found 410.2026.<smiles>C/C=C(\CC)c1ccccc1-c1ccccc1P</smiles>

(E)-(2'-(Hex-3-en-3-yl)-[1,1'-biphenyl]-2-yl)diphenylphosphane (3bf). Following the general procedure A using 2-(diphenylphosphino)biphenyl $(67.7 \mathrm{mg}, 0.2 \mathrm{mmol}$ ), and 3-hexyne ( $23 \mu \mathrm{L}, 0.2 \mathrm{mmol}$ ), the residue was purified by flash chromatography on silica gel (heptane- $\left.\mathrm{Et}_{2} \mathrm{O}=99: 1\right)$ to afford $3 \mathbf{b f}(62.2 \mathrm{mg}, 72 \%)$ as colorless oil.

${ }^{1} \mathrm{H}$ NMR (CDCl $\left.3,400 \mathrm{MHz}\right): \delta 7.40-7.29(\mathrm{~m}, 13 \mathrm{H}), 7.24-7.16(\mathrm{~m}, 3 \mathrm{H}), 7.06$ (ddd, J $=7.5,5.8,3.0 \mathrm{~Hz}, 1 \mathrm{H}), 6.81(\mathrm{dt}, J=6.8,1.3 \mathrm{~Hz}, 1 \mathrm{H}), 5.44(\mathrm{t}, J=7.2 \mathrm{~Hz}, 1 \mathrm{H}), 2.15-1.95(\mathrm{~m}, 4 \mathrm{H}), 0.91$ (td, $J=7.5,4.2 \mathrm{~Hz}, 6 \mathrm{H})$.

${ }^{13} \mathrm{C}\left\{{ }^{1} \mathrm{H}\right\}$ NMR $\left(\mathrm{CDCl}_{3}, 101 \mathrm{MHz}\right): \delta 148.5(\mathrm{~d}, J=32.7 \mathrm{~Hz}), 143.0,141.6,139.5(\mathrm{~d}, J=7.2 \mathrm{~Hz}), 139.0(\mathrm{~d}, J=$ $13.8 \mathrm{~Hz}$ ), 138.0 (d, J = 12.2 Hz), 136.3 (d, J = 12.2 Hz), 134.7 (d, J = 2.3 Hz), 133.9 (d, J = 19.9 Hz), 133.3 (d, $J=19.1 \mathrm{~Hz}$ ), 132.9 (d, J = 1.9 Hz), 131.2 (d, J = 4.9 Hz), 130.7 (d, $J=5.8 \mathrm{~Hz}), 129.8,128.4$ (d, $J=1.8$ $\mathrm{Hz}), 128.3(\mathrm{~d}, J=3.3 \mathrm{~Hz}), 128.2(\mathrm{~d}, J=4.9 \mathrm{~Hz}), 128.0,127.2(\mathrm{~d}, J=21.0 \mathrm{~Hz}), 125.3,23.8,21.3,14.2,13.5$ $(\mathrm{d}, J=2.0 \mathrm{~Hz})$.

${ }^{31} \mathrm{P}\left\{{ }^{1} \mathrm{H}\right\} \mathrm{NMR}\left(\mathrm{CDCl}_{3}, 162 \mathrm{MHz}\right): \delta-14.9(\mathrm{~s})$.

HRMS (ESI) m/z: [M+H] $]^{+}$Calcd for $\mathrm{C}_{30} \mathrm{H}_{42} \mathrm{P}$ 433.3024; Found 433.3025.

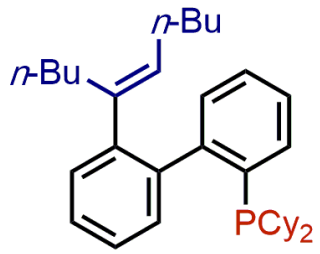
Following the general procedure A using 2-(dicyclohexylphosphino)biphenyl (70.1 $\mathrm{mg}, 0.2 \mathrm{mmol}$ ), and 5-decyne ( $36 \mu \mathrm{L}, 0.2 \mathrm{mmol}$ ), the residue was purified by flash chromatography on silica gel (heptane- $\mathrm{Et}_{2} \mathrm{O}=99: 1$ ) to afford 3ag (63.4 $\mathrm{mg}, 65 \%)$ as colorless oil. 
${ }^{1} \mathrm{H}$ NMR $\left(\mathrm{CDCl}_{3}, 400 \mathrm{MHz}\right): \delta 7.57(\mathrm{~d}, J=7.0 \mathrm{~Hz}, 1 \mathrm{H}), 7.34-7.22(\mathrm{~m}, 6 \mathrm{H}), 7.12(\mathrm{~d}, J=7.4 \mathrm{~Hz}, 1 \mathrm{H}), 5.40$ $(\mathrm{t}, J=7.2 \mathrm{~Hz}, 1 \mathrm{H}), 2.13-1.61(\mathrm{~m}, 17 \mathrm{H}), 1.35-1.10(\mathrm{~m}, 17 \mathrm{H}), 0.91(\mathrm{t}, J=6.9 \mathrm{~Hz}, 3 \mathrm{H}), 0.82(\mathrm{t}, J=6.9 \mathrm{~Hz}$, $3 \mathrm{H})$.

${ }^{13} \mathrm{C}\left\{{ }^{1} \mathrm{H}\right\}$ NMR $\left(\mathrm{CDCl}_{3}, 101 \mathrm{MHz}\right): \delta 149.9(\mathrm{~d}, J=31.4 \mathrm{~Hz}), 143.6,141.4,140.1,135.0(\mathrm{~d}, J=22.3 \mathrm{~Hz})$, $132.7,132.1,131.8$ (d, $J=3.9 \mathrm{~Hz}), 131.0,129.9,127.5,127.0,126.1,125.1,37.0(\mathrm{~d}, J=15.8 \mathrm{~Hz})$, $34.0(\mathrm{~d}, J=14.8 \mathrm{~Hz}), 31.9,30.9,30.7(\mathrm{~d}, J=13.4 \mathrm{~Hz}), 30.5$ (d, $J=17.8 \mathrm{~Hz}), 30.1,29.9,28.0,27.7(\mathrm{~d}, J$ $=15.0 \mathrm{~Hz}$ ), $27.2,26.5(\mathrm{~d}, J=12.6 \mathrm{~Hz}), 22.5(\mathrm{~d}, J=7.2 \mathrm{~Hz}), 14.0$.

${ }^{31} \mathrm{P}\left\{{ }^{1} \mathrm{H}\right\}$ NMR $\left(\mathrm{CDCl}_{3}, 162 \mathrm{MHz}\right): \delta-11.4$ (s).

HRMS (ESI) $\mathrm{m} / \mathrm{z}$ : [M+H] ${ }^{+}$Calcd for $\mathrm{C}_{34} \mathrm{H}_{50} \mathrm{P}$ 489.3645; Found 489.3645.

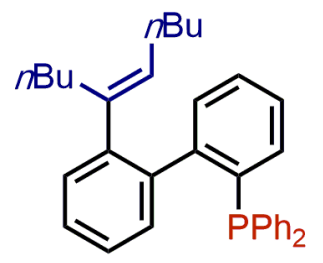
Following the general procedure A using 2-(diphenylphosphino)biphenyl (67.7 $\mathrm{mg}, 0.2 \mathrm{mmol}$ ) and 5-decyne ( $36 \mu \mathrm{L}, 0.2 \mathrm{mmol})$, the residue was purified by flash chromatography on silica gel (heptane-Et $\left.{ }_{2} \mathrm{O}=99: 1\right)$ to afford $3 \mathrm{bg}(62.6 \mathrm{mg}, 67 \%)$ as colorless oil.

${ }^{1} \mathrm{H}$ NMR (CDCl, $\left.400 \mathrm{MHz}\right): \delta 7.40-7.25(\mathrm{~m}, 13 \mathrm{H}), 7.19$ (ddd, $\left.J=9.6,7.0,3.6 \mathrm{~Hz}, 3 \mathrm{H}\right), 7.11-6.98(\mathrm{~m}$, $1 \mathrm{H}), 6.77(\mathrm{~d}, J=7.5 \mathrm{~Hz}, 1 \mathrm{H}), 5.47(\mathrm{t}, J=7.3 \mathrm{~Hz}, 1 \mathrm{H}), 2.13-1.92(\mathrm{~m}, 4 \mathrm{H}), 1.36-1.10(\mathrm{~m}, 8 \mathrm{H}), 0.96-0.81$ $(\mathrm{m}, 6 \mathrm{H})$.

${ }^{13} \mathrm{C}\left\{{ }^{1} \mathrm{H}\right\}$ NMR $\left(\mathrm{CDCl}_{3}, 101 \mathrm{MHz}\right): \delta 148.5(\mathrm{~d}, J=32.8 \mathrm{~Hz}), 143.4,140.8,139.4(\mathrm{~d}, J=7.2 \mathrm{~Hz}), 139.0(\mathrm{~d}, J=$ $13.5 \mathrm{~Hz}$ ), 138.0 (d, $J=12.3 \mathrm{~Hz}$ ), 136.2 (d, $J=12.2 \mathrm{~Hz}$ ), 134.7 (d, $J=2.3 \mathrm{~Hz}$ ), 133.9 (d, $J=19.9 \mathrm{~Hz}$ ), 133.3 (d, $J=19.0 \mathrm{~Hz}$ ), 132.1 (d, J = 1.8 Hz), 131.2 (d, J = 4.8 Hz), 130.8 (d, J = 5.8 Hz), 129.7, 128.4 (d, J = 3.4 $\mathrm{Hz}$ ), 128.3, 128.2, 128.1 (d, J = 3.3 Hz), 127.9, 127.3, 127.0, 125.2, 31.9, 30.8 (d, J= 1.4 Hz), 30.6, 27.9, 22.7, 22.3, 14.1 .

${ }^{31} \mathrm{P}\left\{{ }^{1} \mathrm{H}\right\}$ NMR $\left(\mathrm{CDCl}_{3}, 162 \mathrm{MHz}\right): \delta-14.8(\mathrm{~s})$.

HRMS (ESI) m/z: [M+H] $]^{+}$Calcd for $\mathrm{C}_{34} \mathrm{H}_{38} \mathrm{P}$ 477.2706; Found 477.2706.<smiles>C(=C(c1ccccc1)c1cccc(-c2ccccc2)c1C(=Cc1ccccc1)c1ccccc1)c1ccccc1</smiles>

(2',6'-Bis((E)-1,2-diphenylvinyl)-[1,1'-biphenyl]-2-yl)dicyclohexylphosphane (4aa). Following the general procedure B using 2-(dicyclohexylphosphino)biphenyl (70.1 $\mathrm{mg}, 0.2 \mathrm{mmol})$ and diphenylacetylene $(108.3 \mathrm{mg}, 0.6 \mathrm{mmol})$, the residue was purified by flash chromatography on silica gel (heptane- $\mathrm{Et}_{2} \mathrm{O}=99: 1$ ) to afford 4aa (110.2 $\mathrm{mg}, 78 \%$ ) as white solid; $\mathrm{mp}=208-210^{\circ} \mathrm{C}$. Recrystallization by diffusion method from $\mathrm{CH}_{2} \mathrm{Cl}_{2}$ solution with $n$-hexane has afforded single crystal to analyze by $X$-ray diffraction. 
${ }^{1} \mathrm{H} \mathrm{NMR}\left(\mathrm{CDCl}_{3}, 400 \mathrm{MHz}\right): \delta 7.45(\mathrm{~s}, 3 \mathrm{H}), 7.28(\mathrm{~d}, J=3.4 \mathrm{~Hz}, 1 \mathrm{H}), 7.10-6.93(\mathrm{~m}, 17 \mathrm{H}), 6.86-6.76(\mathrm{~m}$, $1 \mathrm{H}), 6.70(\mathrm{~d}, J=7.5 \mathrm{~Hz}, 4 \mathrm{H}), 6.63(\mathrm{~s}, 1 \mathrm{H}), 6.10(\mathrm{t}, J=7.6 \mathrm{~Hz}, 1 \mathrm{H}), 5.53(\mathrm{dd}, J=7.9,3.8 \mathrm{~Hz}, 1 \mathrm{H}), 2.00-$ $1.56(\mathrm{~m}, 11 \mathrm{H}), 1.42-1.14(\mathrm{~m}, 11 \mathrm{H})$.

${ }^{13} \mathrm{C}\left\{{ }^{1} \mathrm{H}\right\} \operatorname{NMR}\left(\mathrm{CDCl}_{3}, 101 \mathrm{MHz}\right): \delta 145.1(\mathrm{~d}, J=30.6 \mathrm{~Hz}), 144.6(\mathrm{~d}, J=1.9 \mathrm{~Hz}), 143.8,141.5,141.0,137.3$, $135.6(d, J=17.7 \mathrm{~Hz}), 132.2(\mathrm{~d}, J=5.9 \mathrm{~Hz}), 131.6,131.0,129.6,129.5,127.8,127.6,127.2,126.5(\mathrm{~d}, J=$ $4.2 \mathrm{~Hz}), 125.5,124.9,34.5(\mathrm{~d}, J=15.8 \mathrm{~Hz}), 31.8(\mathrm{~d}, J=17.6 \mathrm{~Hz}), 29.4(\mathrm{~d}, J=10.9 \mathrm{~Hz}), 27.6(\mathrm{~d}, J=12.0$ $\mathrm{Hz}), 27.4(\mathrm{~d}, J=7.9 \mathrm{~Hz}), 26.4$.

${ }^{31} \mathrm{P}\left\{{ }^{1} \mathrm{H}\right\}$ NMR $\left(\mathrm{CDCl}_{3}, 162 \mathrm{MHz}\right): \delta-7.7(\mathrm{~s})$ [presence of 1a phosphine oxide at $\left.45.4 \mathrm{ppm}(<5 \%)\right]$.. HRMS (ESI) m/z: [M+H] $]^{+}$Calcd for $\mathrm{C}_{52} \mathrm{H}_{52} \mathrm{P}$ 707.3801; Found 707.3803.<smiles>C(=C(c1ccccc1)c1cccc(-c2ccccc2)c1C(=Cc1ccccc1)c1ccccc1)c1ccccc1</smiles>
(2',6'-Bis((E)-1,2-diphenylvinyl)-[1,1'-biphenyl]-2-yl)diphenylphosphane (4ba). Following the general procedure B using 2-(diphenylphosphino)biphenyl (67.7 mg, $0.2 \mathrm{mmol})$ and diphenylacetylene $(108.3 \mathrm{mg}, 0.6 \mathrm{mmol})$, the residue was purified by flash chromatography on silica gel (heptane- $\mathrm{Et}_{2} \mathrm{O}=99: 1$ ) to afford 4 ba $(83.3$ $\mathrm{mg}, 60 \%)$ as white solid; $\mathrm{mp}=213-215^{\circ} \mathrm{C}$.

${ }^{1} \mathrm{H}$ NMR $\left(\mathrm{CDCl}_{3}, 400 \mathrm{MHz}\right): \delta 7.54-7.45(\mathrm{~m}, 3 \mathrm{H}), 7.32-7.27(\mathrm{~m}, 6 \mathrm{H}), 7.21(\mathrm{td}, J=7.4,1.6 \mathrm{~Hz}, 4 \mathrm{H}), 7.14$ $-7.11(\mathrm{~m}, 1 \mathrm{H}), 7.09-6.97(\mathrm{~m}, 12 \mathrm{H}), 6.83(\mathrm{td}, J=7.6,1.3 \mathrm{~Hz}, 1 \mathrm{H}), 6.73-6.69(\mathrm{~m}, 4 \mathrm{H}), 6.67-6.64(\mathrm{~m}$, $4 \mathrm{H}), 6.35(\mathrm{~s}, 2 \mathrm{H}), 6.27$ (t, J = 7.5 Hz, 1H), 5.74 (ddd, J = 7.9, 4.7, $1.3 \mathrm{~Hz}, 1 \mathrm{H}$ ).

${ }^{13} \mathrm{C}\left\{{ }^{1} \mathrm{H}\right\}$ NMR (CDCl, $\left.101 \mathrm{MHz}\right): \delta 146.1,145.8,145.0(\mathrm{~d}, J=1.9 \mathrm{~Hz}), 142.9,141.2,140.4(\mathrm{~d}, J=5.8 \mathrm{~Hz})$, $139.1(\mathrm{~d}, J=13.4 \mathrm{~Hz}), 137.1,135.5(\mathrm{~d}, J=10.0 \mathrm{~Hz}), 134.8$ (d, $J=2.8 \mathrm{~Hz}), 133.4(\mathrm{~d}, J=18.9 \mathrm{~Hz}$ ), 131.7, $131.2(\mathrm{~d}, J=6.7 \mathrm{~Hz}), 130.6,129.6$ (d, $J=5.5 \mathrm{~Hz}), 128.3(\mathrm{~d}, J=6.2 \mathrm{~Hz}), 128.0,127.7,127.5,127.4,126.9$, $126.5,126.4,126.0$.

${ }^{31} \mathrm{P}\left\{{ }^{1} \mathrm{H}\right\}$ NMR $\left(\mathrm{CDCl}_{3}, 162 \mathrm{MHz}\right): \delta-15.0$ (s) [presence of $\mathbf{1 b}$ phosphine oxide at $47.4 \mathrm{ppm}(<5 \%)$ ].

HRMS (ESI) m/z: [M+H] $]^{+}$Calcd for $\mathrm{C}_{52} \mathrm{H}_{40} \mathrm{P}$ 695.2862; Found 695.2860.<smiles>C(=C(c1ccccc1)c1cccc(C(=Cc2ccccc2)c2ccccc2)c1-c1ccccc1)c1ccccc1</smiles>

$190^{\circ} \mathrm{C}$.

(2',6'-Bis((E)-1,2-diphenylvinyl)-[1,1'-biphenyl]-2-yl)diisopropylphosphane (4ca). Following the general procedure $B$ using 2-(di-(isopropyl)phosphino)biphenyl $(54.1 \mathrm{mg}, 0.2 \mathrm{mmol}$ ) and diphenylacetylene (108.3 $\mathrm{mg}, 0.6 \mathrm{mmol}$ ), the residue was purified by flash chromatography on silica gel (heptane- $\left.\mathrm{Et}_{2} \mathrm{O}=99: 1\right)$ to afford $\mathbf{4} \mathrm{ca}(92.7 \mathrm{mg}, 74 \%)$ as white solid; $\mathrm{mp}=188$ -

${ }^{1} \mathrm{H}$ NMR $\left(\mathrm{CDCl}_{3}, 400 \mathrm{MHz}\right): \delta 7.52(\mathrm{brs}, 3 \mathrm{H}), 7.09-7.01(\mathrm{~m}, 9 \mathrm{H}), 6.93(\mathrm{t}, J=7.7 \mathrm{~Hz}, 9 \mathrm{H}), 6.73-6.63(\mathrm{~m}$, $2 \mathrm{H}), 6.62-6.58(\mathrm{~m}, 4 \mathrm{H}), 6.05(\mathrm{~s}, 1 \mathrm{H}), 5.44(\mathrm{dd}, J=8.0,3.9 \mathrm{~Hz}, 1 \mathrm{H}), 1.22-1.04(\mathrm{~m}, 14 \mathrm{H})$. 
${ }^{13} \mathrm{C}\left\{{ }^{1} \mathrm{H}\right\}$ NMR $\left(\mathrm{CDCl}_{3}, 101 \mathrm{MHz}\right): \delta \backslash 144.7$ (d, $\left.J=1.6 \mathrm{~Hz}\right), 144.5,144.2,144.1,144.0,137.3,132.1,131.7$, 131.5, 130.9, 130.6 (d, J = 5.9 Hz), 129.6, 129.4 (d, J = $2.3 \mathrm{~Hz}$ ), 129.3, 127.7, 126.5, 125.5, 125.1, 23.1 $(\mathrm{d}, J=13.2 \mathrm{~Hz}), 22.6,18.5(\mathrm{~d}, J=9.1 \mathrm{~Hz})$.

${ }^{31} \mathrm{P}\left\{{ }^{1} \mathrm{H}\right\}$ NMR $\left(\mathrm{CDCl}_{3}, 162 \mathrm{MHz}\right): \delta-1.1(\mathrm{~s})$.

HRMS (ESI) m/z: [M+H] $]^{+}$Calcd for $\mathrm{C}_{46} \mathrm{H}_{44} \mathrm{P}$ 627.3175; Found 627.3181.<smiles>CN(C)c1cc(/C(=C/c2ccccc2)c2ccccc2)c(-c2ccccc2)c(/C(=C/c2ccccc2)c2ccccc2)c1</smiles>
2'-(Dicyclohexylphosphanyl)-2,6-bis((E)-1,2-diphenylvinyl)-N,N-dimethyl[1,1'-biphenyl]-4-amine (4ga). Following the general procedure B using 2(dicyclohexylphosphino)-4'-dimethylamimo-biphenyl (78.8 mg, $0.2 \mathrm{mmol}$ ) and diphenylacetylene $(108.3 \mathrm{mg}, 0.6 \mathrm{mmol})$, the residue was purified by flash chromatography on silica gel (heptane- $\mathrm{Et}_{2} \mathrm{O}=97: 3$ ) to afford 4ga (105.1mg, 70\%) as pale yellow solid; $\mathrm{mp}=141-143^{\circ} \mathrm{C}$.

${ }^{1} \mathrm{H} \mathrm{NMR}\left(\mathrm{CDCl}_{3}, 400 \mathrm{MHz}\right): \delta 7.23(\mathrm{~d}, J=7.7 \mathrm{~Hz}, 1 \mathrm{H}), 7.06-6.92(\mathrm{~m}, 17 \mathrm{H}), 6.83(\mathrm{~s}, 2 \mathrm{H}), 6.75-6.66(\mathrm{~m}$, $6 \mathrm{H}), 5.98(\mathrm{t}, J=7.6 \mathrm{~Hz}, 1 \mathrm{H}), 5.39(\mathrm{dd}, J=7.9,4.0 \mathrm{~Hz}, 1 \mathrm{H}), 3.06(\mathrm{~s}, 6 \mathrm{H}), 1.96-1.54(\mathrm{~m}, 11 \mathrm{H}), 1.46-1.10$ $(\mathrm{m}, 11 \mathrm{H})$.

${ }^{13} \mathrm{C}\left\{{ }^{1} \mathrm{H}\right\}$ NMR $\left(\mathrm{CDCl}_{3}, 101 \mathrm{MHz}\right): \delta 148.9,145.6,145.1(\mathrm{~d}, J=14.0 \mathrm{~Hz}), 141.4,137.5,136.3(\mathrm{~d}, J=19.0$ $\mathrm{Hz}$ ), $133.3(\mathrm{~d}, J=4.4 \mathrm{~Hz}$ ), 133.2, 131.4, 130.8, 129.8, 129.5, 129.4, 127.6 (d, J = 4.2 Hz), 126.4, 125.3, 124.4, 115.1, 40.6, 34.7 (d, $J=15.8 \mathrm{~Hz}$ ), 31.7 (d, $J=17.5 \mathrm{~Hz}$ ), 29.6 (d, $J=11.5 \mathrm{~Hz}$ ), 27.6 (d, $J=12.0 \mathrm{~Hz}$ ), $27.4(\mathrm{~d}, J=8.1 \mathrm{~Hz}), 26.4$.

${ }^{31} \mathrm{P}\left\{{ }^{1} \mathrm{H}\right\}$ NMR $\left(\mathrm{CDCl}_{3}, 162 \mathrm{MHz}\right): \delta-8.1(\mathrm{~s})$.

HRMS (ESI) m/z: [M+H] $]^{+}$Calcd for $\mathrm{C}_{54} \mathrm{H}_{57} \mathrm{NP} 750.4223$; Found 750.4228.<smiles>C(=C(c1ccccc1)c1cccc(-c2ccccc2)c1-n1cccc1-c1ccccc1)c1ccccc1</smiles>

1-(2,6-Bis((E)-1,2-diphenylvinyl)phenyl)-2-(diphenylphosphanyl)-1H-pyrrole (4ha). Following the general procedure B using N-phenylpyrrol-2yldiphenylphosphine (65.6 mg, $0.2 \mathrm{mmol})$, and diphenylacetylene (108.3 mg, 0.6 $\mathrm{mmol}$ ), the residue was purified by flash chromatography on silica gel (heptane$\left.\mathrm{Et}_{2} \mathrm{O}=97: 3\right)$ to afford $4 \mathrm{ha}(102.5 \mathrm{mg}, 75 \%)$ as pale yellow solid; $\mathrm{mp}=203-205^{\circ} \mathrm{C}$.

${ }^{1}{ }^{H}$ NMR $\left(\mathrm{CDCl}_{3}, 400 \mathrm{MHz}\right): \delta 7.51-7.39(\mathrm{~m}, 7 \mathrm{H}), 7.29-7.23(\mathrm{~m}, 2 \mathrm{H}), 7.22-7.11(\mathrm{~m}, 10 \mathrm{H}), 7.10-7.00$ $(\mathrm{m}, 6 \mathrm{H}), 6.96(\mathrm{dt}, J=6.8,1.5 \mathrm{~Hz}, 4 \mathrm{H}), 6.70-6.60(\mathrm{~m}, 4 \mathrm{H}), 6.37(\mathrm{~s}, 2 \mathrm{H}), 6.24(\mathrm{dd}, J=3.6,1.5 \mathrm{~Hz}, 1 \mathrm{H}), 5.82$ (ddd, $J=4.1,2.6,1.4 \mathrm{~Hz}, 1 \mathrm{H}), 5.71-5.68(\mathrm{~m}, 1 \mathrm{H})$.

${ }^{13} \mathrm{C}\left\{{ }^{1} \mathrm{H}\right\}$ NMR (CDCl, $\left.101 \mathrm{MHz}\right): \delta 144.4(\mathrm{~d}, J=2.0 \mathrm{~Hz}), 140.2,139.5,139.1(\mathrm{~d}, J=6.0 \mathrm{~Hz}), 137.3(\mathrm{~d}, J=$ $1.8 \mathrm{~Hz}), 137.0,133.4,133.1,131.1$ (d, $J=1.6 \mathrm{~Hz}), 130.9,129.7,129.6,128.3,128.2,128.1,128.0,127.9$, $127.8,127.6(\mathrm{~d}, J=2.8 \mathrm{~Hz}), 127.5,127.0,126.6,119.4$ (d, $J=3.4 \mathrm{~Hz}), 109.0$.

${ }^{31} \mathrm{P}\left\{{ }^{1} \mathrm{H}\right\}$ NMR $\left(\mathrm{CDCl}_{3}, 162 \mathrm{MHz}\right): \delta-33.9(\mathrm{~s})$.

HRMS (ESI) m/z: $[\mathrm{M}+\mathrm{H}]^{+}$Calcd for $\mathrm{C}_{50} \mathrm{H}_{39} \mathrm{NP}$ : 684.2814; Found 684.2811.

$$
S-13
$$




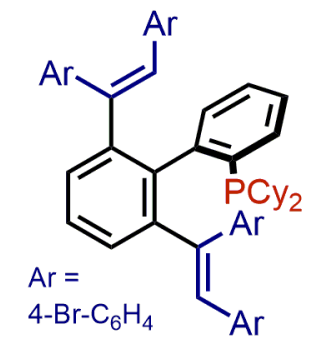

(2',6'-Bis((E)-1,2-bis(4-bromophenyl)vinyl)-[1,1'-biphenyl]-2yl)dicyclohexylphosphane (4ab). Following the general procedure B using 2(dicyclohexylphosphino)biphenyl (70.1 $\mathrm{mg}, 0.2 \mathrm{mmol})$, and Bis(4bromophenyl)acetylene $(201.6 \mathrm{mg}, 0.6 \mathrm{mmol})$, the residue was purified by flash chromatography on silica gel (heptane-Et $\left.{ }_{2} \mathrm{O}=99: 1\right)$ to afford $4 \mathrm{ab}(149.2 \mathrm{mg}, 73 \%)$ as white solid; $m p=220-222{ }^{\circ} \mathrm{C}$.

${ }^{1} \mathrm{H} \mathrm{NMR}\left(\mathrm{CDCl}_{3}, 400 \mathrm{MHz}\right): \delta 7.43(\mathrm{~s}, 3 \mathrm{H}), 7.29-7.24(\mathrm{~m}, 1 \mathrm{H}), 7.19(\mathrm{~d}, J=8.5 \mathrm{~Hz}, 4 \mathrm{H}), 7.16-7.11(\mathrm{~m}$, $4 \mathrm{H}), 6.88(\mathrm{t}, J=7.5 \mathrm{~Hz}, 1 \mathrm{H}), 6.78(\mathrm{~d}, J=8.3 \mathrm{~Hz}, 4 \mathrm{H}), 6.52(\mathrm{~d}, J=8.2 \mathrm{~Hz}, 6 \mathrm{H}), 6.23(\mathrm{t}, J=7.6 \mathrm{~Hz}, 1 \mathrm{H}), 5.57$ (ddd, $J=7.9,3.8,1.3 \mathrm{~Hz}, 1 \mathrm{H}), 1.96-1.54(\mathrm{~m}, 11 \mathrm{H}), 1.37-1.12(\mathrm{~m}, 11 \mathrm{H})$.

${ }^{13} \mathrm{C}\left\{{ }^{1} \mathrm{H}\right\}$ NMR (CDCl $\left.3,101 \mathrm{MHz}\right): \delta$ 144.8, 144.5, $143.8(\mathrm{~d}, J=1.8 \mathrm{~Hz}), 143.1,140.8,139.9,135.7,132.2$, $131.8(\mathrm{~d}, J=2.6 \mathrm{~Hz}), 131.2,131.1,131.0,130.9,127.6,125.7,125.5,121.0,120.8,34.2(\mathrm{~d}, J=15.8 \mathrm{~Hz})$, 31.9, 31.7 (d, $J=17.5 \mathrm{~Hz}$ ), $29.2(\mathrm{~d}, J=10.3 \mathrm{~Hz}), 27.6(\mathrm{~d}, J=12.3 \mathrm{~Hz}), 27.3(\mathrm{~d}, J=8.0 \mathrm{~Hz}$ ), 26.4.

${ }^{31} \mathrm{P}\left\{{ }^{1} \mathrm{H}\right\}$ NMR $\left(\mathrm{CDCl}_{3}, 162 \mathrm{MHz}\right): \delta-7.3(\mathrm{~s})$.

HRMS (ESI) m/z: [M+H] $]^{+}$Calcd for $\mathrm{C}_{52} \mathrm{H}_{48} \mathrm{Br}_{4} \mathrm{P}$ 1019.0222; Found 1019.0222.

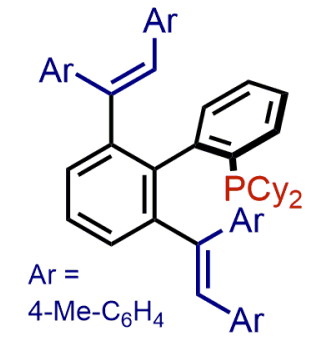

$171^{\circ} \mathrm{C}$.

(2',6'-Bis((E)-1,2-di-p-tolylvinyl)-[1,1'-biphenyl]-2-yl)dicyclohexylphosphane (4ac). Following the general procedure B using 2(dicyclohexylphosphino)biphenyl (70.1 mg, $0.2 \mathrm{mmol}$ ), and 1,2-di-p-tolylethyne (124.5 $\mathrm{mg}, 0.6 \mathrm{mmol})$, the residue was purified by flash chromatography on silica gel (heptane-Et $\left.{ }_{2} \mathrm{O}=99: 1\right)$ to afford $4 \mathrm{ac}(111.3 \mathrm{mg}, 73 \%)$ as white solid; $\mathrm{mp}=169$ -

${ }^{1} \mathrm{H} \mathrm{NMR}\left(\mathrm{CDCl}_{3}, 400 \mathrm{MHz}\right): \delta 7.38(\mathrm{~s}, 3 \mathrm{H}), 7.27(\mathrm{~s}, 1 \mathrm{H}), 6.89-6.81(\mathrm{~m}, 13 \mathrm{H}), 6.64(\mathrm{~d}, J=7.7 \mathrm{~Hz}, 4 \mathrm{H}), 6.53$ $(\mathrm{s}, 2 \mathrm{H}), 6.17(\mathrm{t}, J=7.5 \mathrm{~Hz}, 1 \mathrm{H}), 5.75(\mathrm{dd}, J=8.0,3.8 \mathrm{~Hz}, 1 \mathrm{H}), 2.30(\mathrm{~s}, 6 \mathrm{H}), 2.24(\mathrm{~s}, 6 \mathrm{H}), 1.97-1.55(\mathrm{~m}$, $11 \mathrm{H}), 1.41-1.11(\mathrm{~m}, 11 \mathrm{H})$.

${ }^{13} \mathrm{C}\left\{{ }^{1} \mathrm{H}\right\}$ NMR (CDCl $\left.3,101 \mathrm{MHz}\right): \delta$ 145.6, 145.4, $144.7(\mathrm{~d}, J=1.9 \mathrm{~Hz}), 142.6,140.8$ (d, $\left.J=4.7 \mathrm{~Hz}\right), 138.7$, $136.0(\mathrm{~d}, J=4.8 \mathrm{~Hz}), 135.4(\mathrm{~d}, J=17.7 \mathrm{~Hz}), 134.7,132.5(\mathrm{~d}, J=5.8 \mathrm{~Hz}), 131.5,131.2,130.7,129.6,129.3$, $128.4,128.3,126.9,125.4,124.9,34.4$ (d, $J=15.6 \mathrm{~Hz}), 31.9,31.7$ (d, $J=17.5 \mathrm{~Hz}), 29.4$ (d, J = 11.0 Hz), $27.6(\mathrm{~d}, J=12.2 \mathrm{~Hz}), 27.4(\mathrm{~d}, J=7.7 \mathrm{~Hz}), 26.5,21.3,21.1$.

${ }^{31} \mathrm{P}\left\{{ }^{1} \mathrm{H}\right\}$ NMR $\left(\mathrm{CDCl}_{3}, 162 \mathrm{MHz}\right): \delta-8.2(\mathrm{~s})$.

HRMS (ESI) m/z: [M+H] ${ }^{+}$Calcd for $\mathrm{C}_{56} \mathrm{H}_{60} \mathrm{P}$ 763.4427; Found 763.4426. 


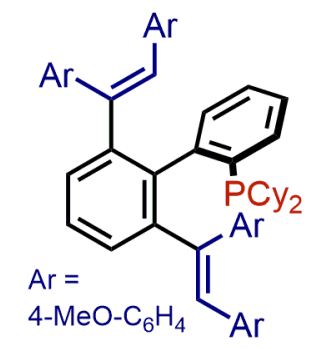

(2',6'-Bis((E)-1,2-bis(4-methoxyphenyl)vinyl)-[1,1'-biphenyl]-2yl)dicyclohexylphosphane (4ad). Following the general procedure B using 2(dicyclohexylphosphino)biphenyl (70.1 $\mathrm{mg}, 0.2 \mathrm{mmol})$, and 1,2-bis(3methoxyphenyl)ethyne $(143.4 \mathrm{mg}, 0.6 \mathrm{mmol})$, the residue was purified by flash chromatography on silica gel (heptane- $\mathrm{Et}_{2} \mathrm{O}=99: 1$ ), the residue was purified by flash chromatography on silica gel (heptane- $\mathrm{Et}_{2} \mathrm{O}=99: 1$ ) to afford 4 ad $(124.1 \mathrm{mg}, 75 \%$ ) as white solid; $\mathrm{mp}=189-191^{\circ} \mathrm{C}$.

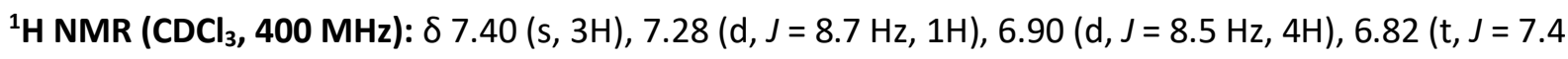
$\mathrm{Hz}, 1 \mathrm{H}), 6.64-6.53(\mathrm{~m}, 12 \mathrm{H}), 6.50(\mathrm{~s}, 2 \mathrm{H}), 6.21(\mathrm{t}, J=7.5 \mathrm{~Hz}, 1 \mathrm{H}), 5.62(\mathrm{dd}, J=7.5,3.6 \mathrm{~Hz}, 1 \mathrm{H}), 3.78(\mathrm{~s}$, $6 \mathrm{H}), 3.73(\mathrm{~s}, 6 \mathrm{H}), 1.99-1.53(\mathrm{~m}, 11 \mathrm{H}), 1.45-1.01(\mathrm{~m}, 11 \mathrm{H})$.

${ }^{13} \mathrm{C}\left\{{ }^{1} \mathrm{H}\right\}$ NMR ( $\left.\mathrm{CDCl}_{3}, 101 \mathrm{MHz}\right): \delta$ 158.3, 158.0, 145.7, 145.4, $144.9(\mathrm{~d}, J=1.8 \mathrm{~Hz}), 141.5,140.8,135.5$, $135.3,134.3,132.4$ (d, $J=6.0 \mathrm{~Hz}$ ), 131.5, 130.8, 130.6, 130.3, 127.0, 125.2, 124.8, 113.3, 113.0, 55.2, 55.1, 34.5 (d, $J=15.7 \mathrm{~Hz}$ ), 31.8 (d, $J=17.3 \mathrm{~Hz}), 29.4$ (d, $J=10.8 \mathrm{~Hz}$ ), 27.6 (d, $J=12.1 \mathrm{~Hz}$ ), 27.4 (d, $J=7.8$ $\mathrm{Hz}), 26.5$.

${ }^{31} \mathrm{P}\left\{{ }^{1} \mathrm{H}\right\} \mathrm{NMR}\left(\mathrm{CDCl}_{3}, 162 \mathrm{MHz}\right): \delta-8.1(\mathrm{~s})$.

HRMS (ESI) m/z: $[\mathrm{M}+\mathrm{H}]^{+}$Calcd for $\mathrm{C}_{56} \mathrm{H}_{60} \mathrm{O}_{4} \mathrm{P}$ 827.4223; Found 827.4219.

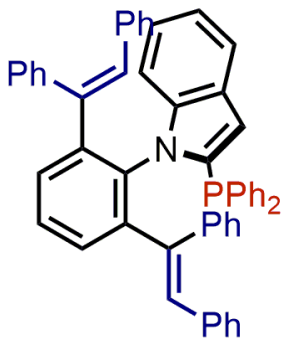

${ }^{1}{ }_{\mathrm{H}}$ NMR $\left(\mathrm{CDCl}_{3}, 400 \mathrm{MHz}\right): \delta 7.53-7.38(\mathrm{~m}, 8 \mathrm{H}), 7.30-7.23(\mathrm{~m}, 3 \mathrm{H}), 7.53-7.38(\mathrm{~m}, 8 \mathrm{H}), 7.21-7.16$ $(\mathrm{m}, 5 \mathrm{H}), 6.74-6.69(\mathrm{~m}, 4 \mathrm{H}), 6.60(\mathrm{~s}, 1 \mathrm{H}), 6.38-6.29(\mathrm{~m}, 5 \mathrm{H}), 6.24(\mathrm{~s}, 2 \mathrm{H})$.

${ }^{13} \mathrm{C}\left\{{ }^{1} \mathrm{H}\right\}$ NMR (CDCl $\left.3,101 \mathrm{MHz}\right): \delta 145.1(\mathrm{~d}, J=1.8 \mathrm{~Hz}), 139.9(\mathrm{~d}, J=3.5 \mathrm{~Hz}), 139.7,139.6,137.9(\mathrm{~d}, J=$ $7.3 \mathrm{~Hz}), 136.8,134.6$ (d, $J=1.8 \mathrm{~Hz}), 133.7,133.5,131.2$ (d, $J=1.9 \mathrm{~Hz}), 131.1,129.4,129.2,128.5,128.4$, $128.3(\mathrm{~d}, J=1.6 \mathrm{~Hz}), 127.8,127.4(\mathrm{~d}, J=1.4 \mathrm{~Hz}), 127.3,126.8,126.4,122.2,119.7,118.9,113.4(\mathrm{~d}, J=$ $3.5 \mathrm{~Hz}), 111.7(\mathrm{~d}, J=1.9 \mathrm{~Hz})$.

${ }^{31} \mathrm{P}\left\{{ }^{1} \mathrm{H}\right\}$ NMR $\left(\mathrm{CDCl}_{3}, 162 \mathrm{MHz}\right): \delta-31.7$ (s).

HRMS (ESI) m/z: [M+H] $]^{+}$Calcd for $\mathrm{C}_{54} \mathrm{H}_{41} \mathrm{NP}$ 734.2972; Found 734.2976.। 


\section{3. $\mathrm{Rh}(\mathrm{l})$-Catalyzed C-H Bond Alkenylation: Reaction on $1 \mathrm{mmol}$ Scale}

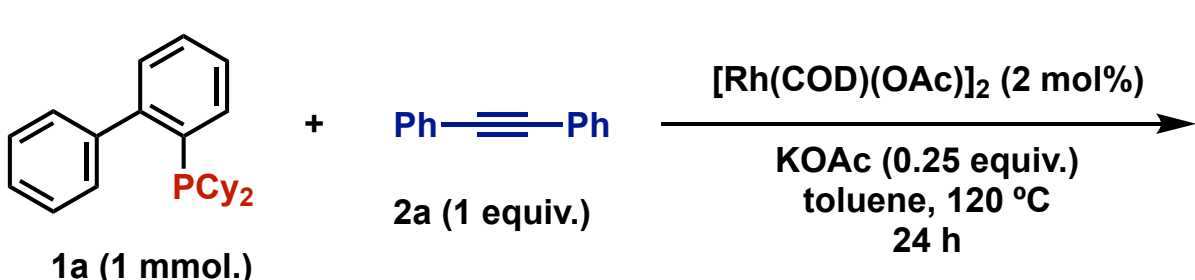<smiles>Pc1ccccc1-c1ccccc1-c1ccccc1P</smiles>

3aa. $81 \%$

Scheme S1. Rhodium(I)-Catalyzed Ortho' C-H Bond Alkenylation of JPhos (1 mmol scale)

To a $15 \mathrm{~mL}$ oven dried Schlenk tube, KOAc ( $24 \mathrm{mg}, 0.25 \mathrm{mmol}, 0.25$ equiv.), JPhos ( $250 \mathrm{mg}, 1 \mathrm{mmol}, 1$ equiv.), toluene $(5 \mathrm{~mL})$, diphenylacetylene (178 mg, $1 \mathrm{mmol}, 1$ equiv) and $[\mathrm{Rh}(\mathrm{OAc})(\mathrm{COD})]_{2}(11.5 \mathrm{mg}$, $0.02 \mathrm{mmol}, 2 \mathrm{~mol} \%)$ were successively added. The reaction mixture was evacuated by vacuum-argon cycles ( 5 times) and stirred at $120^{\circ} \mathrm{C}$ (oil bath temperature) for 24 hours. After cooling the reaction at room temperature and concentration, the residue was purified by flash chromatography on silica gel (heptane-Et ${ }_{2} \mathrm{O}=99: 1$ ) to afford 3aa (420 mg, 81\%).<smiles>O=[PH](O)c1ccccc1-c1ccccc1</smiles>

$1 \mathrm{a}(1 \mathrm{mmol}$.)

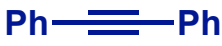

2a (3 equiv.)

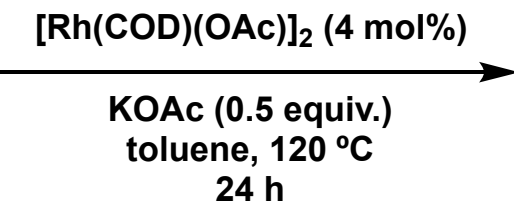

$24 \mathrm{~h}$

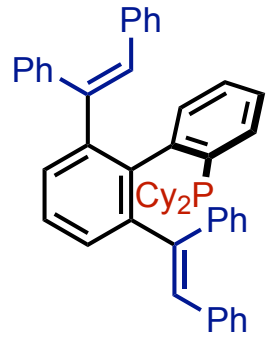

4aa. $77 \%$

Scheme S2. Rhodium(I)-Catalyzed Two-Fold ortho', ortho' C-H Bonds Alkenylation JPhos (1 mmol scale)

To a $15 \mathrm{~mL}$ oven dried Schlenk tube, KOAc ( $48 \mathrm{mg}, 0.5 \mathrm{mmol}, 0.5$ equiv.), JPhos ( $250 \mathrm{mg}, 1 \mathrm{mmol}, 1$ equiv.), toluene ( $5 \mathrm{~mL})$, diphenylacetylene ( $534 \mathrm{mg}, 3 \mathrm{mmol}, 3$ equiv) and $[\mathrm{Rh}(\mathrm{OAc})(\mathrm{COD})]_{2}(23 \mathrm{mg}, 0.04$ mmol, 4 mol\%) were successively added. The reaction mixture was evacuated by vacuum-argon cycles ( 5 times) and stirred at $120^{\circ} \mathrm{C}$ (oil bath temperature) for 24 hours. After cooling the reaction at room temperature and concentration, the residue was purified by flash chromatography on silica gel (heptane-Et $2 \mathrm{O}=99: 1$ ) to afford $4 a a(540 \mathrm{mg}, 77 \%)$. 


\section{Preparation and Characterization of the Palladium Complex 5a}<smiles>Pc1ccccc1/C(=C\c1ccccc1)c1ccccc1/C(=C\c1ccccc1)c1ccccc1</smiles>

4aa

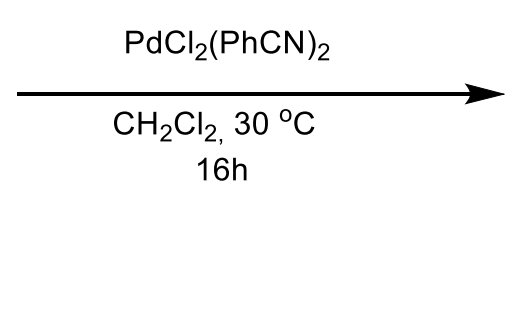

Scheme S3. Preparation of Pd Complex 5a

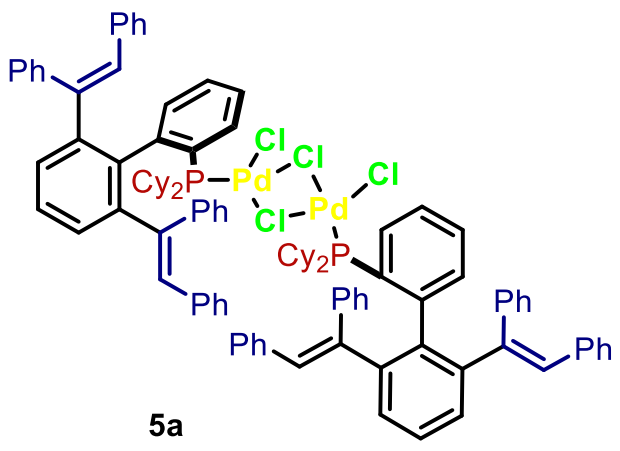

$5 a$

General Procedure C: To a $15 \mathrm{~mL}$ oven dried Schlenk tube, (2',6'-bis((E)-1,2-diphenylvinyl)-[1,1'biphenyl]-2-yl)dicyclohexylphosphane (4aa) $\left(70.7 \mathrm{mg}, 0.1 \mathrm{mmol}, 1\right.$ equiv) and $\mathrm{PdCl}_{2}\left(\mathrm{PhCN}_{2}(38.4 \mathrm{mg}\right.$, $0.1 \mathrm{mmol}, 1$ equiv) were dissolved in degassed $\mathrm{CH}_{2} \mathrm{Cl}_{2}$. The reaction mixture was stirred at $30{ }^{\circ} \mathrm{C}$ (oil bath temperature) for 16 hours. Then the solvent was removed and the residue was washed with pentane to get the orange powder 5 a $(70.7 \mathrm{mg}, 78 \%)$. Recrystallization by diffusion method from $\mathrm{CH}_{2} \mathrm{Cl}_{2}$ solution with $\mathrm{n}$-hexane has afforded single crystal to analyze by $\mathrm{X}$-ray diffraction.

${ }^{1} \mathrm{H}$ NMR $\left(\mathrm{CDCl}_{3}, 400 \mathrm{MHz}\right): \delta 7.60(\mathrm{q}, J=4.0 \mathrm{~Hz}, 3 \mathrm{H}), 7.02-6.78(\mathrm{~m}, 19 \mathrm{H}), 6.73(\mathrm{~s}, 1 \mathrm{H}), 6.63(\mathrm{~d}, J=7.3$ $\mathrm{Hz}, 4 \mathrm{H}), 5.73(\mathrm{~s}, 1 \mathrm{H}), 4.99(\mathrm{~s}, 1 \mathrm{H}), 1.90-1.61(\mathrm{~s}, 13 \mathrm{H}), 1.35-1.10(\mathrm{~m}, 9 \mathrm{H})$.

${ }^{13} \mathrm{C}\left\{{ }^{1} \mathrm{H}\right\}$ NMR $\left(\mathrm{CDCl}_{3}, 101 \mathrm{MHz}\right): \delta 145.1(\mathrm{~d}, J=5.0 \mathrm{~Hz}), 143.3,141.9,140.6,139.9,139.1,137.9,136.9$ , 134.1 (d, $J=7.5 \mathrm{~Hz}$ ), 132.8, 132.2, 129.7 (d, J = 28.7 Hz), 129.1 , $129.0,128.5,128.2,127.7$ (d, J = $18.8 \mathrm{~Hz}$ ), 126.7 (d, J = 9.2 Hz), 125.9 (d, J = 5.8 Hz), 125.3, 124.0 (d, J = 13.8 Hz), 34.2 (d, J = 11.0 Hz), $31.2(\mathrm{~d}, J=2.4 \mathrm{~Hz}), 30.6(\mathrm{~d}, J=8.7 \mathrm{~Hz}), 27.5,27.4(\mathrm{~d}, J=1.8 \mathrm{~Hz}), 27.4$, 25.7 .

${ }^{31} \mathrm{P}\left\{{ }^{1} \mathrm{H}\right\}$ NMR (CDCl, $\left.162 \mathrm{MHz}\right): \delta 67.0(\mathrm{~s})$.

Elemental analysis: calcd (\%) for $\mathrm{C}_{104} \mathrm{H}_{102} \mathrm{Cl}_{4} \mathrm{P}_{2} \mathrm{Pd}_{2}$ (1768.54): $\mathrm{C} 70.63, \mathrm{H} \mathrm{5.81}$; found: $\mathrm{C} 70.45$, H 5.67 . 
$5{ }^{1} \mathrm{H} \mathrm{NMR}\left(\mathrm{CDCl}_{3}, 400 \mathrm{MHz}\right)$

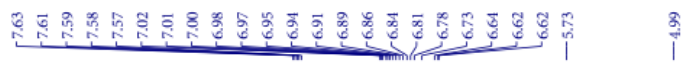

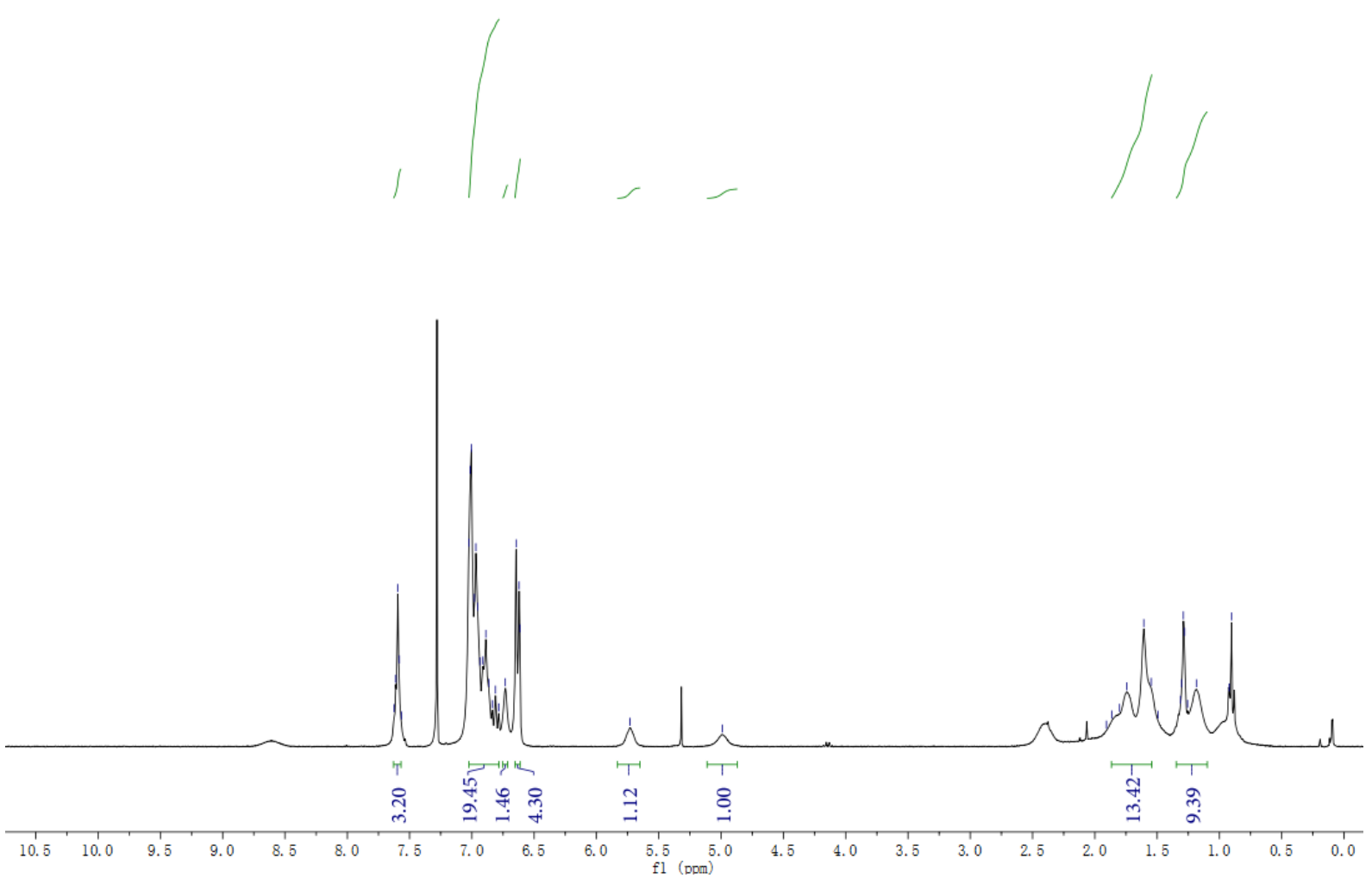

5a ${ }^{13} \mathrm{C}\left\{{ }^{1} \mathrm{H}\right\} \mathrm{NMR}\left(\mathrm{CDCl}_{3}, 101 \mathrm{MHz}\right)$

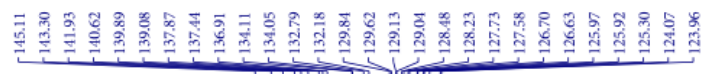

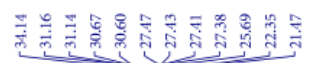
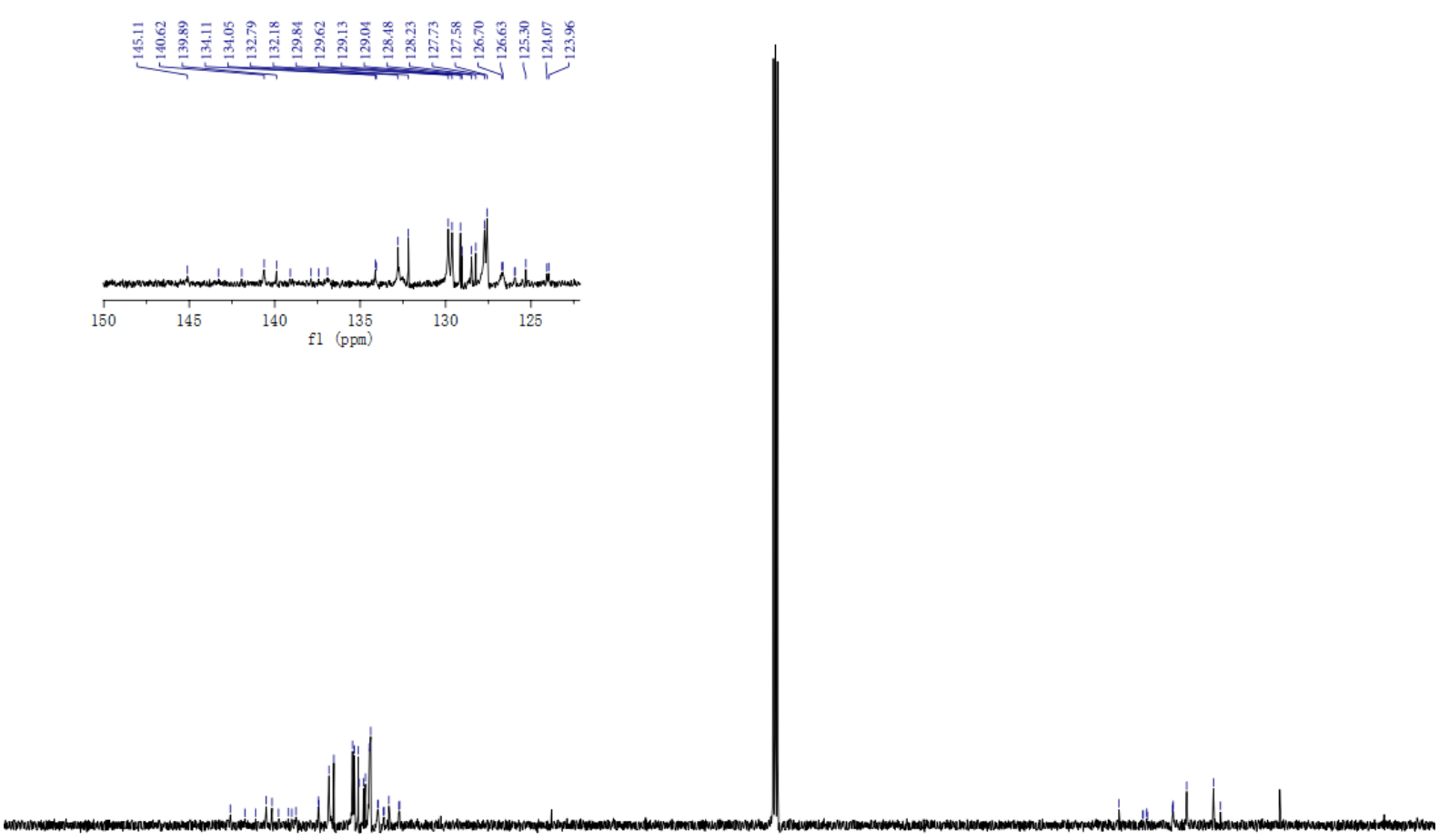

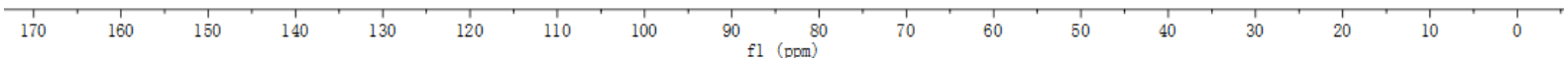

$S-18$ 
$5 \mathbf{a}^{31} \mathrm{P}\left\{{ }^{1} \mathrm{H}\right\} \mathrm{NMR}\left(\mathrm{CDCl}_{3}, 162 \mathrm{MHz}\right)$

$$
\text { i. }
$$

Deco

$: 50$

$200 \quad 150$

100

50

f1 (ppm)

S - 19 
4. Catalytic Evaluation of Phosphine in Pd-Catalyzed Amidation: Procedure and Compound Characterizations

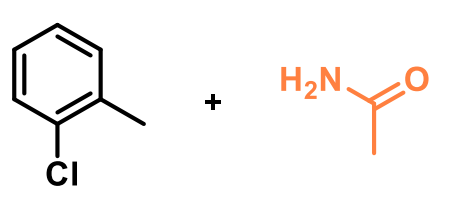

(2 equiv.)
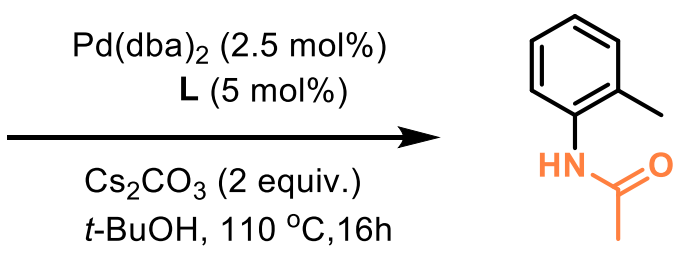

$6 a$

General Procedure D: In oven-dried $15 \mathrm{~mL}$ Schlenk tube, which was equipped with a magnetic stir bar, $\mathrm{Pd}(\mathrm{dba})_{2}$ (4.3 mg, $0.0075 \mathrm{mmol}, 2.5 \mathrm{~mol} \%$ ), biarylphosphine phosphine $(0.015 \mathrm{mmol}, 5 \mathrm{~mol} \%$.), amide (0.6 mmol), aryl chloride $(0.3 \mathrm{mmol}) \mathrm{Cs}_{2} \mathrm{CO}_{3}(195 \mathrm{mg}, 0.6 \mathrm{mmol})$ and t-BuOH $(1.5 \mathrm{~mL})$ were successfully added under argon. The solution was heated to $110{ }^{\circ} \mathrm{C}$ for $16 \mathrm{~h}$. The reaction was cold done and $n$ dodecane ( $38 \mu \mathrm{L}, 0.3 \mathrm{mmol}$ ) was added to analyze and calculate the yields by GC-analysis.

GC analysis: Performed on a Shimadzu GC-2010 apparatus (Column: INTERCHIM, UPTIBOND 1 PREMIUM, $0.25 \mathrm{~mm}$ ID, $0.25 \mathrm{~mm}, 30.0 \mathrm{~m}$; Gas pressure: $81.7 \mathrm{kPa}$; Total flow: $30.8 \mathrm{~mL}^{\mathrm{min}}{ }^{-1}$; Column flow : $1.07 \mathrm{~mL}$.min -1 ; Velocity: $27.4 \mathrm{~cm} / \mathrm{sec}$; Purge flow: $3.0 \mathrm{~mL}$. $\mathrm{min}^{-1}$; Sprit ratio: 46.0; Injector: 250ㄷ, FID: $280^{\circ} \mathrm{C}$; Column program: starting from $50^{\circ} \mathrm{C}, 2 \mathrm{~min}$ hold, $10^{\circ} \mathrm{C} / \mathrm{min}$ to $240^{\circ} \mathrm{C}, 5 \mathrm{~min}$ hold).

$t_{R}(\min )=11.0($ internal standard), 14.4 (product) 
Table S1. Evaluation of Biarylphosphine in Pd-Catalyzed Amidation of 2-Chlorotoluene with Acetamide

\begin{tabular}{|c|c|c|c|c|c|}
\hline Entry & L structure & $\mathbf{L}$ & $\mathrm{MM}(\mathrm{g} / \mathrm{mol})$ & m Ligand (mg) & Yield of $6 a(\%)^{[a]}$ \\
\hline 1 & & $1 a$ & 350.48 & 5.3 & 0 \\
\hline 2 & & $1 e$ & 364.50 & 5.5 & 0 \\
\hline 3 & & $1 f$ & 393.55 & 5.9 & 0 \\
\hline 4 & & $1 \mathrm{~g}$ & 393.55 & 5.9 & 0 \\
\hline 5 & & $1 \mathbf{i}$ & 403.53 & 6.1 & 0 \\
\hline 6 & & CyXPhos & 476.72 & 7.2 & 8 \\
\hline 7 & & 3ea & 542.31 & 8.2 & 37 \\
\hline 8 & & $3 f a$ & 571.34 & 8.6 & 23 \\
\hline 9 & & 3ia & 581.32 & 8.7 & 15 \\
\hline 10 & & $4 a a$ & 706.38 & 10.6 & 95 \\
\hline 11 & & 4ga & 749.42 & 11.2 & 82 \\
\hline
\end{tabular}

[a] Determined by GC-analysis using $n$-dodecane as internal standard. 
$\underbrace{\infty}_{N}$

$\mathbf{N}$-(o-tolyl)acetamide (6a): Reaction was performed using acetamide (35.4 mg, 0.6 mmol), 2-chlorotoluene (35 $\mu \mathrm{L}, 0.3 \mathrm{mmol}$ ) and $\mathrm{Cs}_{2} \mathrm{CO}_{3}$ (195 mg, $0.6 \mathrm{mmol}$ ), $\mathrm{Pd}(\mathrm{dba})_{2}$ (4.3 mg, $0.0075 \mathrm{mmol}, 2.5 \mathrm{~mol} \%$ ), ligand 4aa (10.6 mg, $0.015 \mathrm{mmol}, 5 \mathrm{~mol} \%$ ) and $t$ $\mathrm{BuOH}(1.5 \mathrm{~mL}$ ), the residue was purified by flash chromatography on silica gel (heptane-EtOAc $=70: 30$ ) to afford $6 a(40.7 \mathrm{mg}, 91 \%)$ as a white solid; $\mathrm{mp}=108-110^{\circ} \mathrm{C} ; \mathrm{GC}: \mathrm{t}_{\mathrm{R}}=8.4 \mathrm{~min}$. $\mathrm{MS}(\mathrm{EI}): \mathrm{m} / \mathrm{z}: 149\left(\mathrm{M}^{+}\right)$. ${ }^{1} \mathrm{H} \mathrm{NMR}\left(\mathrm{CDCl}_{3}, 400 \mathrm{MHz}\right): \delta 7.65(\mathrm{~s}, 1 \mathrm{H}), 7.56(\mathrm{~d}, J=8.0 \mathrm{~Hz}, 1 \mathrm{H}), 7.15(\mathrm{t}, J=7.2 \mathrm{~Hz}, 2 \mathrm{H}), 7.11-7.04(\mathrm{~m}$, $1 \mathrm{H}), 2.20(\mathrm{~s}, 3 \mathrm{H}), 2.12(\mathrm{~s}, 3 \mathrm{H})$.

${ }^{13} \mathrm{C}\left\{{ }^{1} \mathrm{H}\right\}$ NMR $\left(\mathrm{CDCl}_{3}, 101 \mathrm{MHz}\right): \delta$ 169.0, 135.7, 130.6, 130.5, 126.5, 125.6, 124.3, 23.9, 17.8.

The NMR spectra are identical with those reported in the literature. ${ }^{[3]}$<smiles>Cc1ccccc1NC(=O)C(C)(C)C</smiles>

$\boldsymbol{N}$-(o-tolyl)pivalamide (6b): Reaction was performed using pivalamide $(60.7 \mathrm{mg}$, $0.6 \mathrm{mmol}), 2$-chlorotoluene $(35 \mu \mathrm{L}, 0.3 \mathrm{mmol})$ and $\mathrm{Cs}_{2} \mathrm{CO}_{3}(195 \mathrm{mg}, 0.6 \mathrm{mmol})$, $\mathrm{Pd}(\mathrm{dba})_{2}$ (4.3 mg, $\left.0.0075 \mathrm{mmol}, 2.5 \mathrm{~mol} \%\right)$, ligand 4aa (10.6 mg, $0.015 \mathrm{mmol}, 5$ $\mathrm{mol} \%)$ and $\mathrm{t}-\mathrm{BuOH}(1.5 \mathrm{~mL})$, the residue was purified by flash chromatography on silica gel (heptaneEtOAc $=70: 30)$ to afford $6 \mathbf{b}(51.6 \mathrm{mg}, 90 \%)$ as a white solid; $\mathrm{mp}=111-113^{\circ} \mathrm{C} ; \mathrm{GC}: \mathrm{t}_{\mathrm{R}}=9.3 \mathrm{~min}$. $\mathrm{MS}$ (EI): $\mathrm{m} / \mathrm{z}: 191\left(\mathrm{M}^{+}\right)$.

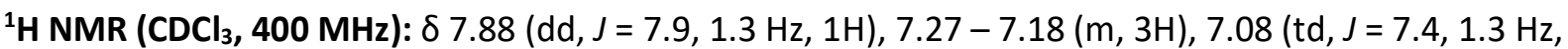
$1 \mathrm{H}), 2.28(\mathrm{~s}, 3 \mathrm{H}), 1.36(\mathrm{~s}, 9 \mathrm{H})$.

${ }^{13} \mathrm{C}\left\{{ }^{1} \mathrm{H}\right\}$ NMR (CDCl $\left.3,101 \mathrm{MHz}\right): \delta 176.5,135.9,130.4,128.7,126.8,124.9,122.8,39.7,27.7,17.6$.

The NMR spectra are identical with those reported in the literature. ${ }^{[4]}$<smiles>CC(C)(C)C(=O)Nc1ccccc1[N+](=O)[O-]</smiles>

$N$-(2-nitrophenyl)pivalamide (6c): Reaction was performed using pivalamide (60.7 mg, $0.6 \mathrm{mmol}$ ), 2-nitrochlorobenzene $\left(47.3 \mathrm{mg}, 0.3 \mathrm{mmol}\right.$ ) and $\mathrm{Cs}_{2} \mathrm{CO}_{3}(195$ $\mathrm{mg}, 0.6 \mathrm{mmol}), \mathrm{Pd}(\mathrm{dba})_{2}(4.3 \mathrm{mg}, 0.0075 \mathrm{mmol}, 2.5 \mathrm{~mol} \%)$, ligand 4aa (10.6 mg, $0.015 \mathrm{mmol}, 5 \mathrm{~mol} \%)$ and t-BuOH $(1.5 \mathrm{~mL})$, the residue was purified by flash chromatography on silica gel (heptane-EtOAc $=70: 30)$ to afford $6 c(53.2 \mathrm{mg}, 80 \%)$ as yellow oil; GC: $\mathrm{t}_{R}=10.3 \mathrm{~min}$. MS (EI): $\mathrm{m} / \mathrm{z}$ : $222\left(\mathrm{M}^{+}\right)$.

${ }^{1} \mathrm{H} \mathrm{NMR}\left(\mathrm{CDCl}_{3}, 400 \mathrm{MHz}\right): \delta 10.70(\mathrm{~s}, 1 \mathrm{H}), 8.80(\mathrm{dd}, J=8.6,1.4 \mathrm{~Hz}, 1 \mathrm{H}), 8.18(\mathrm{dd}, J=8.5,1.6 \mathrm{~Hz}, 1 \mathrm{H})$, $7.69-7.54(m, 1 H), 7.20-7.06(m, 1 H), 1.34(s, 9 H)$.

${ }^{13} \mathrm{C}\left\{{ }^{1} \mathrm{H}\right\}$ NMR $\left(\mathrm{CDCl}_{3}, 101 \mathrm{MHz}\right): \delta$ 177.8, 136.3, 136.0, 135.4, 125.7, 122.9, 122.0, 40.5, 27.4.

HRMS (ESI) m/z: [M+H] $]^{+}$Calcd for $\mathrm{C}_{11} \mathrm{H}_{14} \mathrm{~N}_{2} \mathrm{O}_{3}$ 223.1077; Found 223.1077. 
<smiles>CC(C)(C)C(=O)Nc1ccc([N+](=O)[O-])cc1C#N</smiles>
$\mathbf{N}$-(2-cyano-4-nitrophenyl)pivalamide (6d): Reaction was performed using pivalamide (60.7 mg, $0.6 \mathrm{mmol}$ ), 2-chloro-5-nitrobenzonitrile (54.8 mg, 0.3 $\mathrm{mmol}$ ) and $\mathrm{Cs}_{2} \mathrm{CO}_{3}(195 \mathrm{mg}, 0.6 \mathrm{mmol}), \mathrm{Pd}(\mathrm{dba})_{2}(4.3 \mathrm{mg}, 0.0075 \mathrm{mmol}, 2.5$ mol\%), ligand 4aa (10.6 mg, $0.015 \mathrm{mmol}, 5 \mathrm{~mol} \%)$ and t-BuOH $(1.5 \mathrm{~mL})$, the residue was purified by flash chromatography on silica gel (heptane-EtOAc $=70: 30)$ to afford $6 \mathbf{d}(61.5 \mathrm{mg}, 83 \%)$ as a white solid; $\mathrm{mp}=230-232^{\circ} \mathrm{C} ; \mathrm{GC}: \mathrm{t}_{\mathrm{R}}=13.6 \mathrm{~min}$. $\mathbf{M S}(\mathrm{EI}): \mathrm{m} / \mathrm{z}: 247\left(\mathrm{M}^{+}\right)$.

${ }^{1} \mathrm{H} \mathrm{NMR}\left(\mathrm{CDCl}_{3}, 400 \mathrm{MHz}\right): \delta 11.38(\mathrm{~s}, 1 \mathrm{H}), 9.13(\mathrm{~d}, J=2.6 \mathrm{~Hz}, 1 \mathrm{H}), 8.56(\mathrm{dd}, J=9.0,2.7 \mathrm{~Hz}, 1 \mathrm{H}), 7.86(\mathrm{~d}$, $J=9.0 \mathrm{~Hz}, 1 \mathrm{H}), 1.55(\mathrm{~s}, 9 \mathrm{H})$.

${ }^{13} \mathrm{C}\left\{{ }^{1} \mathrm{H}\right\}$ NMR $\left(\mathrm{CDCl}_{3}, 101 \mathrm{MHz}\right): \delta$ 166.0, 162.9, 153.2, 145.4, 129.4, 128.6, 122.8, 120.6, 38.0, 28.2.

HRMS (ESI) m/z: [M+H] $]^{+}$Calcd for $\mathrm{C}_{12} \mathrm{H}_{13} \mathrm{~N}_{3} \mathrm{O}_{3}$ 248.1030; Found 248.1029 .

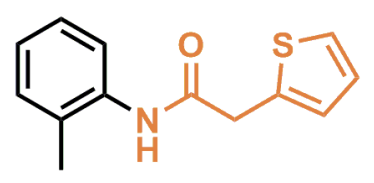

2-(Thiophen-2-yl)-N-(o-tolyl)acetamide (6e): Reaction was performed using 2-(thiophen-2-yl)acetamide ( $84.7 \mathrm{mg}, 0.6 \mathrm{mmol})$, 2-chlorotoluene (35 $\mu \mathrm{L}, 0.3 \mathrm{mmol}$ ) and $\mathrm{Cs}_{2} \mathrm{CO}_{3}(195 \mathrm{mg}, 0.6 \mathrm{mmol}), \mathrm{Pd}(\mathrm{dba})_{2}(4.3 \mathrm{mg}, 0.0075$ $\mathrm{mmol}, 2.5 \mathrm{~mol} \%)$, ligand 4 aa $(10.6 \mathrm{mg}, 0.015 \mathrm{mmol}, 5 \mathrm{~mol} \%)$ and t-BuOH $(1.5 \mathrm{~mL})$, the residue was purified by flash chromatography on silica gel (heptane-EtOAc $=70: 30)$ to afford $6 \mathrm{e}(58.2 \mathrm{mg}, 84 \%)$ as a white solid; $\mathrm{mp}=146-148^{\circ} \mathrm{C} ; \mathrm{GC}: \mathrm{t}_{\mathrm{R}}=12.5 \mathrm{~min}$. $\mathrm{MS}$ (EI): $\mathrm{m} / \mathrm{z}: 231\left(\mathrm{M}^{+}\right)$.

${ }^{1} \mathrm{H} \mathrm{NMR}\left(\mathrm{CDCl}_{3}, 400 \mathrm{MHz}\right): \delta 7.96-7.86(\mathrm{~m}, 1 \mathrm{H}), 7.37-7.32(\mathrm{~m}, 1 \mathrm{H}), 7.28(\mathrm{~s}, 1 \mathrm{H}), 7.22(\mathrm{td}, J=7.7,1.7$ $\mathrm{Hz}, 1 \mathrm{H}), 7.14(\mathrm{~d}, J=6.6 \mathrm{~Hz}, 1 \mathrm{H}), 7.08(\mathrm{dd}, J=9.9,2.4 \mathrm{~Hz}, 3 \mathrm{H}), 4.00(\mathrm{~s}, 2 \mathrm{H}), 2.02(\mathrm{~s}, 3 \mathrm{H})$.

${ }^{13} \mathrm{C}\left\{{ }^{1} \mathrm{H}\right\}$ NMR $\left(\mathrm{CDCl}_{3}, 101 \mathrm{MHz}\right): \delta 167.9,136.0,135.4,130.4,128.4,128.1,127.7,126.8,126.2,125.2$, $122.3,38.4,17.2$.

The NMR spectra are identical with those reported in the literature. ${ }^{[5]}$<smiles>O=C(Cc1cccs1)Nc1ccccc1C(F)(F)F</smiles>

2-(Thiophen-2-yl)-N-(2-(trifluoromethyl)phenyl)acetamide (6f): Reaction was performed using 2-(thiophen-2-yl)acetamide $(84.7 \mathrm{mg}, 0.6 \mathrm{mmol}), 2$ chlorobenzotrifluoride ( $40 \mu \mathrm{L}, 0.3 \mathrm{mmol}$ ) and $\mathrm{Cs}_{2} \mathrm{CO}_{3}(195 \mathrm{mg}, 0.6 \mathrm{mmol}$ ), $\mathrm{Pd}(\mathrm{dba})_{2}$ (4.3 mg, $0.0075 \mathrm{mmol}, 2.5 \mathrm{~mol} \%$ ), ligand 4aa (10.6 mg, $0.015 \mathrm{mmol}, 5 \mathrm{~mol} \%$ ) and t-BuOH (1.5 $\mathrm{mL}$ ), the residue was purified by flash chromatography on silica gel (heptane-EtOAc $=70: 30$ ) to afford 6f (68.4 mg, 80\%) as a yellow solid; $\mathrm{mp}=109-111^{\circ} \mathrm{C} ; \mathrm{GC}: \mathrm{t}_{\mathrm{R}}=11.0 \mathrm{~min} . \mathbf{M S}(\mathrm{EI}): \mathrm{m} / \mathrm{z}: 285\left(\mathrm{M}^{+}\right)$.

${ }^{1} \mathrm{H} \mathrm{NMR}\left(\mathrm{CDCl}_{3}, 400 \mathrm{MHz}\right): \delta 8.28(\mathrm{~d}, J=8.3 \mathrm{~Hz}, 1 \mathrm{H}), 7.78-7.68(\mathrm{~m}, 1 \mathrm{H}), 7.56(\mathrm{t}, J=7.6 \mathrm{~Hz}, 2 \mathrm{H}), 7.36$ (dd, $J=4.9,1.5 \mathrm{~Hz}, 1 \mathrm{H}), 7.22(\mathrm{t}, J=7.7 \mathrm{~Hz}, 1 \mathrm{H}), 7.12-7.08(\mathrm{~m}, 2 \mathrm{H}), 4.02(\mathrm{~s}, 2 \mathrm{H})$.

${ }^{13} \mathrm{C}\left\{{ }^{1} \mathrm{H}\right\}$ NMR $\left(\mathrm{CDCl}_{3}, 101 \mathrm{MHz}\right): \delta 168.1,135.1,134.6,132.9,128.3,127.8,126.5,126.0(\mathrm{q}, J=5.2 \mathrm{~Hz})$, $124.5,123.8,123.7(q, J=273.6 \mathrm{~Hz}), 38.5$. 
${ }^{19} \mathrm{~F}\left\{{ }^{1} \mathrm{H}\right\}$ NMR $\left(\mathrm{CDCl}_{3}, 471 \mathrm{MHz}\right): \delta 3-61.2$ (s).

The NMR spectra are identical with those reported in the literature. ${ }^{[5]}$

\section{Solid-State Structures}

\section{Crystal measurement for 3aa:}

Crystallization by diffusion method from $\mathrm{CH}_{2} \mathrm{Cl}_{2}$ solution with $n$-hexane has afforded single crystal to analyze by X-ray diffraction.

The single crystal X-ray diffraction studies were carried out on D8 VENTURE Bruker AXS diffractometer equipped with a (CMOS) PHOTON 100 detector, $\left[{ }^{*}\right]$, Mo-Ka radiation $(\lambda=0.71073 \AA$, multilayer monochromator), $T=150 \mathrm{~K}$; monoclinic $\mathrm{P} 2_{1} / \mathrm{c}(\mathrm{I} . \mathrm{T} . \# 14), \mathrm{a}=9.7682(18), b=15.168(3), c=20.187(4)$ $\AA, \beta=90.385(8)^{\circ}, V=2990.9(10) \AA^{3} . Z=4, d=1.174 \mathrm{~g} . \mathrm{cm}^{-3}, \mu=0.117 \mathrm{~mm}^{-1}$. The structure was solved by dual-space algorithm using the SHELXT program [1], and then refined with full-matrix least-squares methods based on $\mathrm{F}^{2}$ (SHELXL) [2]. All non-hydrogen atoms were refined with anisotropic atomic displacement parameters. $\mathrm{H}$ atoms were finally included in their calculated positions and treated as riding on their parent atom with constrained thermal parameters. A final refinement on $\mathrm{F}^{2}$ with 6853 unique intensities and 352 parameters converged at $\omega R_{F}^{2}=0.1375\left(R_{F}=0.0527\right)$ for 4937 observed reflections with $\mathrm{I}>2 \sigma(\mathrm{I})$.

[1] G. M. Sheldrick, Acta Cryst. A71 (2015) 3-8

[2] Sheldrick G.M., Acta Cryst. C71 (2015) 3-8

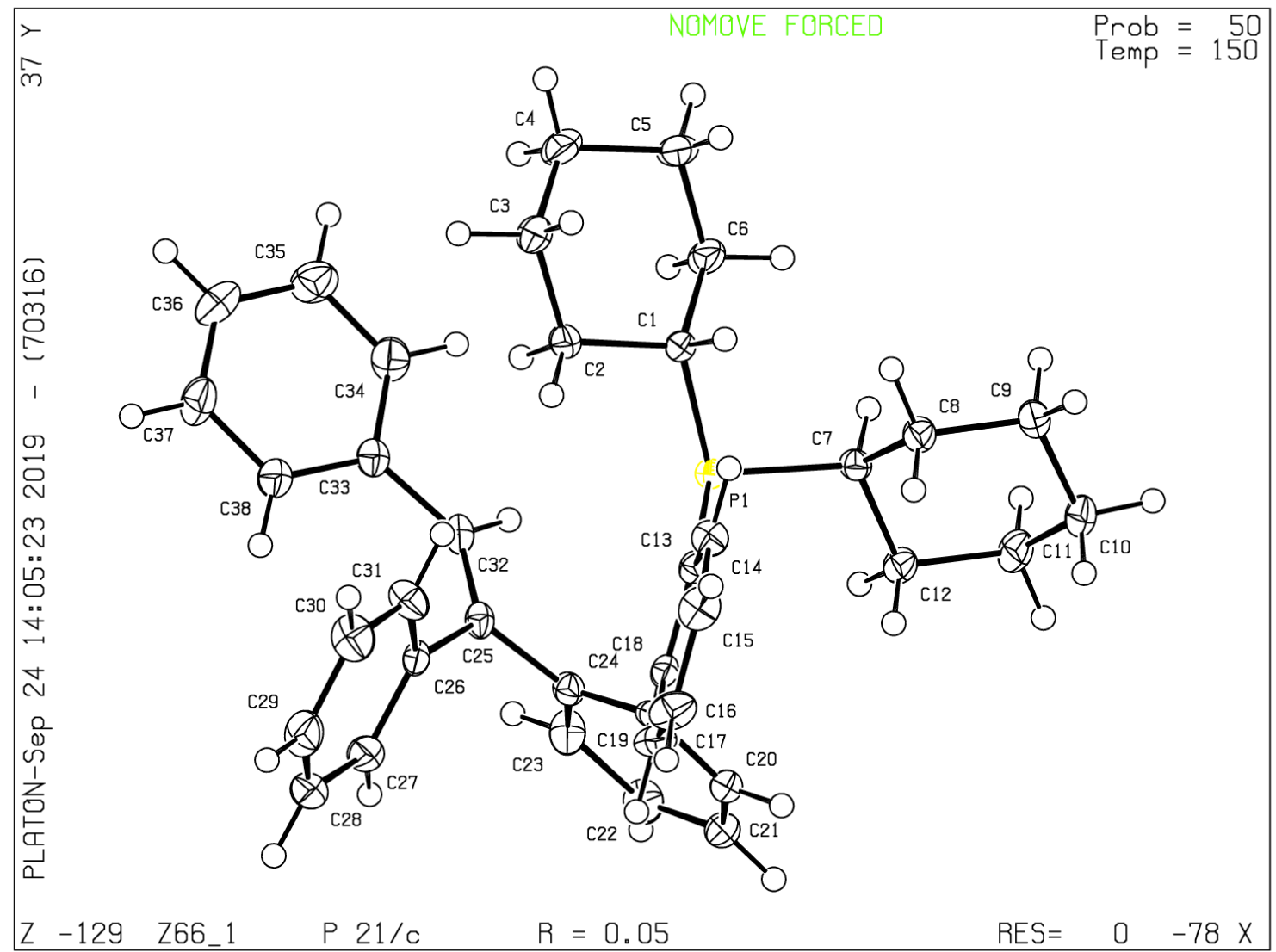

Figure S1. ORTEP diagram of the solid-state molecular structure of 3aa. Thermal ellipsoids are at the $50 \%$ probability level. (CCDC 968419). 
Table S1. Crystal data and structure refinement for 3aa.

\begin{tabular}{|c|c|}
\hline Empirical formula & $\mathrm{C} 38 \mathrm{H} 41 \mathrm{P}$ \\
\hline Formula weight & $528.68 \mathrm{~g} / \mathrm{mol}$ \\
\hline Temperature & $150 \mathrm{~K}$ \\
\hline Wavelength & $0.71073 \AA$ \\
\hline Crystal system, space group & monoclinic, P 21/c \\
\hline \multirow[t]{6}{*}{ Unit cell dimensions } & $a=9.7682(18) \AA$ \\
\hline & $b=15.168(3) \AA$ \\
\hline & $c=20.187(4) \AA$ \\
\hline & $\alpha=90^{\circ}$ \\
\hline & $\beta=90.385(8)^{\circ}$ \\
\hline & $\gamma=90^{\circ}$ \\
\hline Volume & 2990.9(10) А̊3 \\
\hline Z, Calculated density & 4, $1.174 \mathrm{~g} . \mathrm{cm}-3$ \\
\hline Absorption coefficient & $0.117 \mathrm{~mm}-1$ \\
\hline$F(000)$ & 1136 \\
\hline Crystal size & $0.400 \times 0.300 \times 0.220 \mathrm{~mm}$ \\
\hline Crystal color & colourless \\
\hline Theta range for data collection & 2.424 to $27.528^{\circ}$ \\
\hline h_min, h_max & $-12,12$ \\
\hline k_min, k_max & $-19,17$ \\
\hline I_min, I_max & $-26,26$ \\
\hline Reflections collected / unique & $25185 / 6853[\mathrm{R}(\mathrm{int}) \mathrm{a}=0.0884]$ \\
\hline Reflections $[\mid>2 \sigma]$ & 4937 \\
\hline Completeness to theta_max & 0.993 \\
\hline Absorption correction type & multi-scan \\
\hline Max. and min. transmission & $0.975,0.765$ \\
\hline Refinement method & Full-matrix least-squares on F2 \\
\hline Data / restraints / parameters & $6853 / 0 / 352$ \\
\hline bS (Goodness-of-fit) & 0.988 \\
\hline Final $R$ indices $[1>2 \sigma]$ & $R 1 c=0.0527, w R 2 d=0.1375$ \\
\hline $\mathrm{R}$ indices (all data) & $R 1 c=0.0876, w R 2 d=0.1597$ \\
\hline Largest diff. peak and hole & 0.278 and -0.385 e- $. \AA \AA-3$ \\
\hline
\end{tabular}

Table S2. Fractional atomic coordinates, site occupancy (\%) and equivalent isotropic displacement parameters $\left(\AA^{2}\right)$ for 2 aa. $U(e q)$ is defined as one third of the trace of the orthogonalized Uij tensor.
Atom
U11
U22
U33
U23
U13
U12

P1 $0.0144(2) \quad 0.0154(2) \quad 0.0161(3) \quad 0.00092(17) \quad 0.00038(17)-0.00100(17)$

$\begin{array}{llllllll}\text { C1 } & 0.0155(9) & 0.0188(8) & 0.0167(10) & 0.0004(7) & 0.0008(7) & 0.0011(7)\end{array}$

$\begin{array}{llllllll}\mathrm{C} 2 & 0.0195(9) & 0.0259(9) & 0.0180(10) & 0.0032(8) & 0.0025(8) & 0.0015(7)\end{array}$

$\begin{array}{llllllll}\text { C3 } & 0.0167(10) & 0.0387(12) & 0.0272(12) & 0.0061(9) & 0.0055(8) & 0.0040(8)\end{array}$

$\begin{array}{llllllll}\text { C4 } & 0.0244(11) & 0.0405(12) & 0.0396(14) & 0.0124(10) & 0.0103(10) & 0.0146(9)\end{array}$

$\begin{array}{llllllll}\text { C5 } & 0.0259(11) & 0.0421(13) & 0.0350(13) & 0.0196(10) & 0.0086(9) & 0.0156(9)\end{array}$

$\begin{array}{llllllll}\text { C6 } & 0.0211(10) & 0.0266(10) & 0.0315(12) & 0.0096(8) & 0.0085(9) & 0.0055(8)\end{array}$

$\begin{array}{llllllll}\text { C7 } & 0.0153(9) & 0.0213(9) & 0.0179(10) & 0.0044(7) & 0.0016(7) & -0.0005(7)\end{array}$

$\begin{array}{llllllll}\mathrm{C} 8 & 0.0167(9) & 0.0318(11) & 0.0193(11) & -0.0003(8) & 0.0006(8) & 0.0012(8)\end{array}$ 


\begin{tabular}{|c|c|c|c|c|c|c|}
\hline 9 & .0245(10) & $0.0414(12)$ & $0.0175(11)$ & $0.0007(9)$ & $0.0022(8)$ & \\
\hline C10 & $0.0227(10)$ & $0.0409(12)$ & $0.0236(12)$ & $0.0014(9)$ & $0.0085(9)$ & \\
\hline 11 & $0.0175(10)$ & $0.0384(12)$ & $0.0301(12)$ & $0.0004(9)$ & $0.0069(8)$ & $0006(8)$ \\
\hline 12 & $.0154(9)$ & $0.0286(10)$ & $0.0225(11)$ & $-0.0005(8)$ & $0.0012(8)$ & \\
\hline 3 & (8) & $0.0157(8)$ & $0.0184(10)$ & $0.0000(7)$ & $0.0039(7)-c$ & -0.00 \\
\hline 14 & (10) & $0.0222(9)$ & $0.0227(11)$ & $-0.0003(8)$ & $-0.0008(8)$ & -0.00 \\
\hline 15 & $0.0240(10)$ & $0.0202(9)$ & $0.0351(13)$ & $-0.0024(8)$ & $0.0025(9)$ & $-0.0068(8)$ \\
\hline 16 & (11) & $0.0150(9)$ & (14) & $0.0055(8)$ & $0.0016(9)-$ & -0.0 \\
\hline 7 & 10) & $4(10)$ & $5(12)$ & $0.0071(8)$ & $-0.0014(8)$ & $5(8)$ \\
\hline 18 & (9) & 0.01 & 0.01 & -0.00 & $0.0037(7)$ & 0.00 \\
\hline 19 & 9) & & & & -0.0 & \\
\hline 20 & 0.01 & $0.0257(10)$ & 0.0 & $0.002 C$ & $0.0015(8)$ & 0.00 \\
\hline 1 & 0.0 & $0.0386(12)$ & 0.0 & 0.0041 & $0.0017(8)$ & 0.003 \\
\hline 22 & $5(10)$ & $0.0428(12)$ & $0.0279(12)$ & $-0.0026(9)$ & $-0.0013(8)$ & $-0.0077(8)$ \\
\hline 23 & (10) & $8(11)$ & t(11) & $-0.0068(8)$ & $0.0014(8)$ & $-0.0060(8)$ \\
\hline 24 & 0.01 & 0.023 & 0.01 & $0.0024(7)$ & (7) $-c$ & -0.00 \\
\hline 25 & 0.0 & 0.02 & 0.01 & -0.00 & $0.0012(7)$ & -0.003 \\
\hline 26 & 0.01 & $0.0214(9)$ & 0.01 & $-0.0030(7)$ & $0.0039(7)$ & 0.001 \\
\hline 27 & 0.02 & $0.0333(11)$ & $0.0249(12)$ & $0.0044(8)$ & $0.0017(8)$ & $0.0040(8)$ \\
\hline 28 & (12) & 1(12) & $3(12)$ & $0.0108(9)$ & $0.0060(9)$ & $0.0108(9)$ \\
\hline 29 & (12) & $0.0274(11)$ & $0.0360(13)$ & $0.0074(9)$ & $0.0147(10)$ & ) $-0.0011(9)$ \\
\hline 30 & 0.02 & $0.0348(12)$ & $0.0328(13)$ & $0.0018(9)$ & $0.0025(9)$ & $-0.0101(9)$ \\
\hline 31 & $3(10)$ & $0.0317(11)$ & $0.0205(11)$ & $0.0032(8)$ & $-0.0029(8)$ & $-0.0064(8)$ \\
\hline 32 & $0.0198(9)$ & $0.0246(10)$ & $0.0171(10)$ & $-0.0037(8)$ & $0.0036(7)$ & $-0.0030(7)$ \\
\hline 33 & 0.01 & 0.01 & 0.01 & $-0.0049(7) \quad-$ & $-0.0003(7)$ & $-0.0039(7)$ \\
\hline 34 & (12) & $0.0234(10)$ & $8(12)$ & $0.0003(9)$ & $0.0064(9)$ & $-0.0008(8)$ \\
\hline C35 & $0(13)$ & $0.0234(10)$ & $0.0416(14)$ & $0.0020(9)$ & $0.0008(11)$ & ) $0.0044(9)$ \\
\hline C36 & $0.0232(11)$ & $0.0302(11)$ & $0.0471(15)$ & $-0.0080(10)$ & $0.0015(10)$ & 0) $0.0064(8)$ \\
\hline 37 & $0.0279(11)$ & $0.0370(12)$ & $0.0356(13)$ & $-0.0022(10)$ & $0.0128(10)$ & $0.0017(9)$ \\
\hline & $0 \angle J \angle(10)$ & $.030<(11)$ & $.0 \angle 5 U(11)$ & $0.0025(8)$ & $0.0056(9)$ & $.0033(8)$ \\
\hline
\end{tabular}

Table S3. Bond lengths $[\AA ̊]$ for 3aa.

$\begin{array}{lll}\mathrm{P} 1 & -\mathrm{C} 13=1.8531(18) \\ \mathrm{P} 1 & -\mathrm{C} 1=1.8619(18) \\ \mathrm{P} 1 & -\mathrm{C} 7=1.8675(19) \\ \mathrm{C} 1-\mathrm{C} 6 & =1.530(3) \\ \mathrm{C} 1 & -\mathrm{C} 2=1.535(2) \\ \mathrm{C} 1-\mathrm{H} 1 & =1.0000 \\ \mathrm{C} 2 & -\mathrm{C} 3=1.527(3) \\ \mathrm{C} 2 & -\mathrm{H} 2 \mathrm{~A}=0.9900 \\ \mathrm{C} 2 & -\mathrm{H} 2 \mathrm{~B}=0.9900 \\ \mathrm{C} 3 & -\mathrm{C} 4=1.517(3) \\ \mathrm{C} 3 & -\mathrm{H} 3 \mathrm{~A}=0.9900 \\ \mathrm{C} 3 & -\mathrm{H} 3 \mathrm{~B}=0.9900 \\ \mathrm{C} 4 & -\mathrm{C} 5=1.532(3) \\ \mathrm{C} 4 & -\mathrm{H} 4 \mathrm{~A}=0.9900 \\ \mathrm{C} 4 & -\mathrm{H} 4 \mathrm{~B}=0.9900 \\ \mathrm{C} 5 & -\mathrm{C} 6=1.526(3) \\ \mathrm{C} 5 & -\mathrm{H} 5 \mathrm{~A}=0.9900 \\ \mathrm{C} 5 & -\mathrm{H} 5 \mathrm{~B}=0.9900 \\ \mathrm{C} 6 & -\mathrm{H} 6 \mathrm{~A}=0.9900\end{array}$




$$
\begin{aligned}
& \mathrm{C} 6-\mathrm{H} 6 \mathrm{~B}=0.9900 \\
& \mathrm{C} 7-\mathrm{C} 8=1.524(3) \\
& \mathrm{C} 7-\mathrm{C} 12=1.537(2) \\
& \mathrm{C} 7-\mathrm{H} 7=1.0000 \\
& \mathrm{C} 8-\mathrm{C} 9=1.531(3) \\
& \mathrm{C} 8-\mathrm{H} 8 \mathrm{~A}=0.9900 \\
& \mathrm{C} 8-\mathrm{H} 8 \mathrm{~B}=0.9900 \\
& \mathrm{C} 9-\mathrm{C} 10=1.524(3) \\
& \mathrm{C} 9-\mathrm{H} 9 \mathrm{~A}=0.9900 \\
& \mathrm{C} 9-\mathrm{H} 9 \mathrm{~B}=0.9900 \\
& \mathrm{C} 10-\mathrm{C} 11=1.524(3) \\
& \mathrm{C} 10-\mathrm{H} 10 \mathrm{~A}=0.9900 \\
& \mathrm{C} 10-\mathrm{H} 10 \mathrm{~B}=0.9900 \\
& \mathrm{C} 11-\mathrm{C} 12=1.535(3) \\
& \mathrm{C} 11-\mathrm{H} 11 \mathrm{~A}=0.9900 \\
& \mathrm{C} 11-\mathrm{H} 11 \mathrm{~B}=0.9900 \\
& \mathrm{C} 12-\mathrm{H} 12 \mathrm{~A}=0.9900 \\
& \mathrm{C} 12-\mathrm{H} 12 \mathrm{~B}=0.9900 \\
& \mathrm{C} 13-\mathrm{C} 14=1.405(3) \\
& \mathrm{C} 13-\mathrm{C} 18=1.407(3) \\
& \mathrm{C} 14-\mathrm{C} 15=1.380(3) \\
& \mathrm{C} 14-\mathrm{H} 14=0.9500 \\
& \mathrm{C} 15-\mathrm{C} 16=1.381(3) \\
& \mathrm{C} 15-\mathrm{H} 15=0.9500 \\
& \mathrm{C} 16-\mathrm{C} 17=1.382(3) \\
& \mathrm{C} 16-\mathrm{H} 16=0.9500 \\
& \mathrm{C} 17-\mathrm{C} 18=1.401(3) \\
& \mathrm{C} 17-\mathrm{H} 17=0.9500 \\
& \mathrm{C} 18-\mathrm{C} 19=1.499(2) \\
& \mathrm{C} 19-\mathrm{C} 20=1.401(2) \\
& \mathrm{C} 19-\mathrm{C} 24=1.406(3) \\
& \mathrm{C} 20-\mathrm{C} 21=1.381(3) \\
& \mathrm{C} 2 \mathrm{O}-\mathrm{H} 20=0.9500 \\
& \mathrm{C} 21-\mathrm{C} 22=1.382(3) \\
& \mathrm{C} 21-\mathrm{H} 21=0.9500 \\
& \mathrm{C} 22-\mathrm{C} 23=1.384(3) \\
& \mathrm{C} 22-\mathrm{H} 22=0.9500 \\
& \mathrm{C} 23-\mathrm{C} 24=1.399(3) \\
& \mathrm{C} 23-\mathrm{H} 23=0.9500 \\
& \mathrm{C} 24-\mathrm{C} 25=1.497(2) \\
& \mathrm{C} 25-\mathrm{C} 32=1.335(3) \\
& \mathrm{C} 25-\mathrm{C} 26=1.504(2) \\
& \mathrm{C} 26-\mathrm{C} 31=1.388(3) \\
& \mathrm{C} 26-\mathrm{C} 27=1.388(3) \\
& \mathrm{C} 27-\mathrm{C} 28=1.388(3) \\
& \mathrm{C} 27-\mathrm{H} 27=0.9500 \\
& \mathrm{C} 28-\mathrm{C} 29=1.376(3) \\
& \mathrm{C} 28-\mathrm{H} 28=0.9500 \\
& \mathrm{C} 29-\mathrm{C} 30=1.381(3) \\
& \mathrm{C} 29-\mathrm{H} 29=0.9500 \\
& \mathrm{C} 30-\mathrm{C} 31=1.382(3) \\
& \mathrm{C} 30-\mathrm{H} 30=0.9500
\end{aligned}
$$




$$
\begin{array}{ll}
\mathrm{C} 31-\mathrm{H} 31 & =0.9500 \\
\mathrm{C} 32-\mathrm{C} 33 & =1.479(2) \\
\mathrm{C} 32-\mathrm{H} 32 & =0.9500 \\
\mathrm{C} 33-\mathrm{C} 34 & =1.391(3) \\
\mathrm{C} 33-\mathrm{C} 38 & =1.393(3) \\
\mathrm{C} 34-\mathrm{C} 35 & =1.391(3) \\
\mathrm{C} 34-\mathrm{H} 34 & =0.9500 \\
\mathrm{C} 35-\mathrm{C} 36 & =1.375(3) \\
\mathrm{C} 35-\mathrm{H} 35 & =0.9500 \\
\mathrm{C} 36-\mathrm{C} 37 & =1.377(3) \\
\mathrm{C} 36-\mathrm{H} 36 & =0.9500 \\
\mathrm{C} 37-\mathrm{C} 38 & =1.394(3) \\
\mathrm{C} 37-\mathrm{H} 37 & =0.9500 \\
\mathrm{C} 38-\mathrm{H} 38 & =0.9500
\end{array}
$$

Table S4. Angles [ $\left.{ }^{\circ}\right]$ for $3 a a$.

$\begin{array}{llll}\mathrm{C} 13 & -\mathrm{P} 1 & -\mathrm{C} 1 & =102.34(8) \\ \mathrm{C} 13 & -\mathrm{P} 1 & -\mathrm{C} 7 & =102.89(8) \\ \mathrm{C} 1 & -\mathrm{P} 1 & -\mathrm{C} 7 & =101.03(8) \\ \mathrm{C} 6 & -\mathrm{C} 1 & -\mathrm{C} 2 & =110.23(15) \\ \mathrm{C} 6 & -\mathrm{C} 1 & -\mathrm{P} 1 & =109.90(12) \\ \mathrm{C} 2 & -\mathrm{C} 1 & -\mathrm{P} 1 & =110.67(13) \\ \mathrm{C} 6 & -\mathrm{C} 1 & -\mathrm{H} 1 & =108.70 \\ \mathrm{C} 2 & -\mathrm{C} 1 & -\mathrm{H} 1 & =108.70 \\ \mathrm{P} 1 & -\mathrm{C} 1 & -\mathrm{H} 1 & =108.70 \\ \mathrm{C} 3 & -\mathrm{C} 2 & -\mathrm{C} 1 & =112.58(16) \\ \mathrm{C} 3 & -\mathrm{C} 2 & -\mathrm{H} 2 \mathrm{~A} & =109.10 \\ \mathrm{C} 1 & -\mathrm{C} 2 & -\mathrm{H} 2 \mathrm{~A} & =109.10 \\ \mathrm{C} 3 & -\mathrm{C} 2 & -\mathrm{H} 2 \mathrm{~B} & =109.10 \\ \mathrm{C} 1 & -\mathrm{C} 2 & -\mathrm{H} 2 \mathrm{~B} & =109.10 \\ \mathrm{H} 2 \mathrm{~A} & -\mathrm{C} 2 & -\mathrm{H} 2 \mathrm{~B} & =107.80 \\ \mathrm{C} 4 & -\mathrm{C} 3 & -\mathrm{C} 2 & =111.78(16) \\ \mathrm{C} 4 & -\mathrm{C} 3 & -\mathrm{H} 3 \mathrm{~A} & =109.30 \\ \mathrm{C} 2 & -\mathrm{C} 3 & -\mathrm{H} 3 \mathrm{~A} & =109.30 \\ \mathrm{C} 4 & -\mathrm{C} 3 & -\mathrm{H} 3 \mathrm{~B} & =109.30 \\ \mathrm{C} 2 & -\mathrm{C} 3 & -\mathrm{H} 3 \mathrm{~B} & =109.30 \\ \mathrm{H} 3 \mathrm{~A} & -\mathrm{C} 3 & -\mathrm{H} 3 \mathrm{~B} & =107.90 \\ \mathrm{C} 3 & -\mathrm{C} 4 & -\mathrm{C} 5 & =110.68(17) \\ \mathrm{C} 3 & -\mathrm{C} 4 & -\mathrm{H} 4 \mathrm{~A} & =109.50 \\ \mathrm{C} 5 & -\mathrm{C} 4 & -\mathrm{H} 4 \mathrm{~A} & =109.50 \\ \mathrm{C} 3 & -\mathrm{C} 4 & -\mathrm{H} 4 \mathrm{~B} & =109.50 \\ \mathrm{C} 5 & -\mathrm{C} 4 & -\mathrm{H} 4 \mathrm{~B} & =109.50 \\ \mathrm{H} 4 \mathrm{~A} & -\mathrm{C} 4 & -\mathrm{H} 4 \mathrm{~B} & =108.10 \\ \mathrm{C} 6 & -\mathrm{C} 5 & -\mathrm{C} 4 & =110.85(18) \\ \mathrm{C} 6 & -\mathrm{C} 5 & -\mathrm{H} 5 \mathrm{~A} & =109.50 \\ \mathrm{C} 4 & -\mathrm{C} 5 & -\mathrm{H} 5 \mathrm{~A} & =109.50 \\ \mathrm{C} 6 & -\mathrm{C} 5 & -\mathrm{H} 5 \mathrm{~B} & =109.50 \\ \mathrm{C} 4 & -\mathrm{C} 5 & -\mathrm{H} 5 \mathrm{~B} & =109.50 \\ \mathrm{H} 5 \mathrm{~A} & -\mathrm{C} 5 & -\mathrm{H} 5 \mathrm{~B} & =108.10 \\ \mathrm{C} 5 & -\mathrm{C} 6 & -\mathrm{C} 1 & =111.87(16) \\ \mathrm{C} 5 & -\mathrm{C} 6 & -\mathrm{H} 6 \mathrm{~A} & =109.20 \\ & & & \end{array}$




$$
\begin{aligned}
& \mathrm{C} 1-\mathrm{C} 6-\mathrm{H} 6 \mathrm{~A}=109.20 \\
& \mathrm{C} 5-\mathrm{C} 6-\mathrm{H} 6 \mathrm{~B}=109.20 \\
& \mathrm{C} 1-\mathrm{C} 6-\mathrm{H} 6 \mathrm{~B}=109.20 \\
& \mathrm{H} 6 \mathrm{~A}-\mathrm{C} 6-\mathrm{H} 6 \mathrm{~B}=107.90 \\
& \mathrm{C} 8-\mathrm{C} 7-\mathrm{C} 12=109.63(15) \\
& \mathrm{C} 8-\mathrm{C} 7-\mathrm{P} 1=118.51(12) \\
& \mathrm{C} 12-\mathrm{C} 7-\mathrm{P} 1=110.83(13) \\
& \mathrm{C} 8-\mathrm{C} 7-\mathrm{H} 7=105.60 \\
& \mathrm{C} 12-\mathrm{C} 7-\mathrm{H} 7=105.60 \\
& \mathrm{P} 1-\mathrm{C} 7 \quad-\mathrm{H} 7=105.60 \\
& \mathrm{C} 7-\mathrm{C} 8-\mathrm{C} 9=110.63(15) \\
& \mathrm{C} 7-\mathrm{C} 8-\mathrm{H} 8 \mathrm{~A}=109.50 \\
& \mathrm{C} 9-\mathrm{C} 8-\mathrm{H} 8 \mathrm{~A}=109.50 \\
& \mathrm{C} 7-\mathrm{C} 8-\mathrm{H} 8 \mathrm{~B}=109.50 \\
& \mathrm{C} 9-\mathrm{C} 8-\mathrm{H} 8 \mathrm{~B}=109.50 \\
& \mathrm{H} 8 \mathrm{~A}-\mathrm{C} 8-\mathrm{H} 8 \mathrm{~B}=108.10 \\
& \mathrm{C} 10-\mathrm{C} 9-\mathrm{C} 8=111.95(17) \\
& \mathrm{C} 10-\mathrm{C} 9-\mathrm{H} 9 \mathrm{~A}=109.20 \\
& \mathrm{C} 8-\mathrm{C} 9-\mathrm{H} 9 \mathrm{~A}=109.20 \\
& \mathrm{C} 10-\mathrm{C} 9-\mathrm{H} 9 \mathrm{~B}=109.20 \\
& \mathrm{C} 8-\mathrm{C} 9-\mathrm{H} 9 \mathrm{~B}=109.20 \\
& \mathrm{H} 9 \mathrm{~A}-\mathrm{C} 9-\mathrm{H} 9 \mathrm{~B}=107.90 \\
& \mathrm{C} 11-\mathrm{C} 10-\mathrm{C} 9=111.18(17) \\
& \mathrm{C} 11-\mathrm{C} 10-\mathrm{H} 10 \mathrm{~A}=109.40 \\
& \mathrm{C} 9-\mathrm{C} 10-\mathrm{H} 10 \mathrm{~A}=109.40 \\
& \mathrm{C} 11-\mathrm{C} 10-\mathrm{H} 10 \mathrm{~B}=109.40 \\
& \mathrm{C} 9-\mathrm{C} 10-\mathrm{H} 10 \mathrm{~B}=109.40 \\
& \mathrm{H} 10 \mathrm{~A}-\mathrm{C} 10-\mathrm{H} 10 \mathrm{~B}=108.00 \\
& \mathrm{C} 10-\mathrm{C} 11-\mathrm{C} 12=111.62(16) \\
& \mathrm{C} 10-\mathrm{C} 11-\mathrm{H} 11 \mathrm{~A}=109.30 \\
& \mathrm{C} 12-\mathrm{C} 11-\mathrm{H} 11 \mathrm{~A}=109.30 \\
& \mathrm{C} 10-\mathrm{C} 11-\mathrm{H} 11 \mathrm{~B}=109.30 \\
& \mathrm{C} 12-\mathrm{C} 11-\mathrm{H} 11 \mathrm{~B}=109.30 \\
& \mathrm{H} 11 \mathrm{~A}-\mathrm{C} 11-\mathrm{H} 11 \mathrm{~B}=108.00 \\
& \mathrm{C} 11-\mathrm{C} 12-\mathrm{C} 7 \quad=110.92(16) \\
& \mathrm{C} 11-\mathrm{C} 12-\mathrm{H} 12 \mathrm{~A}=109.50 \\
& \mathrm{C} 7-\mathrm{C} 12-\mathrm{H} 12 \mathrm{~A}=109.50 \\
& \mathrm{C} 11-\mathrm{C} 12-\mathrm{H} 12 \mathrm{~B}=109.50 \\
& \mathrm{C} 7-\mathrm{C} 12-\mathrm{H} 12 \mathrm{~B}=109.50 \\
& \mathrm{H} 12 \mathrm{~A}-\mathrm{C} 12-\mathrm{H} 12 \mathrm{~B}=108.00 \\
& \mathrm{C} 14-\mathrm{C} 13-\mathrm{C} 18=117.85(16) \\
& \mathrm{C} 14-\mathrm{C} 13-\mathrm{P} 1=122.07(14) \\
& \mathrm{C} 18-\mathrm{C} 13-\mathrm{P} 1=120.06(13) \\
& \mathrm{C} 15-\mathrm{C} 14-\mathrm{C} 13=122.23(19) \\
& \mathrm{C} 15-\mathrm{C} 14-\mathrm{H} 14=118.90 \\
& \mathrm{C} 13-\mathrm{C} 14-\mathrm{H} 14=118.90 \\
& \mathrm{C} 14-\mathrm{C} 15-\mathrm{C} 16=119.61(18) \\
& \mathrm{C} 14-\mathrm{C} 15-\mathrm{H} 15=120.20 \\
& \mathrm{C} 16-\mathrm{C} 15-\mathrm{H} 15=120.20 \\
& \mathrm{C} 15-\mathrm{C} 16-\mathrm{C} 17=119.53(18) \\
& \mathrm{C} 15-\mathrm{C} 16-\mathrm{H} 16=120.20 \\
& \mathrm{C} 17-\mathrm{C} 16-\mathrm{H} 16=120.20
\end{aligned}
$$




\begin{tabular}{|c|c|c|c|}
\hline C16 & - C17 & - C18 & $=121.73(19$ \\
\hline C16 & - C17 & $-\mathrm{H} 17$ & $=119.10$ \\
\hline & - C17 & - H17 & $=119.10$ \\
\hline & - C18 & - C13 & $=119.04(17)$ \\
\hline 17 & - C18 & - C19 & $=117.25(16)$ \\
\hline 13 & - C18 & - C19 & $=123.50(16)$ \\
\hline 20 & - C19 & $-C 24$ & $=118.43(17)$ \\
\hline 20 & - C19 & - C18 & $=117.32(16)$ \\
\hline 4 & - C19 & - C18 & $=124.24(16)$ \\
\hline 1 & $-\mathrm{C} 20$ & - C19 & $=122.01(18)$ \\
\hline 1 & - C20 & $-\mathrm{H} 2 \mathrm{O}$ & 9.00 \\
\hline 9 & $-\mathrm{C} 20$ & $-\mathrm{H} 2 \mathrm{O}$ & $=1$ \\
\hline 0 & $-C 21$ & $-C 22$ & $59(18)$ \\
\hline 20 & $-C 21$ & $-\mathrm{H} 21$ & $=12$ \\
\hline 22 & $-C 21$ & $-\mathrm{H} 21$ & $=1$ \\
\hline 21 & $-\mathrm{C} 22$ & $-\mathrm{C} 23$ & 27(19) \\
\hline 21 & $-\mathrm{C} 22$ & $-\mathrm{H} 22$ & \\
\hline 3 & $-\mathrm{C} 22$ & $-\mathrm{H} 22$ & \\
\hline 22 & $-\mathrm{C} 23$ & $-\mathrm{C} 24$ & $=1$ \\
\hline 2 & $-\mathrm{C} 23$ & $-\mathrm{H} 23$ & $=1$ \\
\hline 4 & $-\mathrm{C} 23$ & $-\mathrm{H} 23$ & $=1$ \\
\hline 23 & $-\mathrm{C} 24$ & - C19 & $50(17)$ \\
\hline 23 & $-\mathrm{C} 24$ & $-\mathrm{C} 25$ & 60(17) \\
\hline 19 & $-\mathrm{C} 24$ & $-\mathrm{C} 25$ & $85(16)$ \\
\hline C32 & $-\mathrm{C} 25$ & $-\mathrm{C} 24$ & $3(16)$ \\
\hline C32 & $-\mathrm{C} 25$ & $-\mathrm{C} 26$ & $3(16)$ \\
\hline C24 & $-\mathrm{C} 25$ & $-\mathrm{C} 26$ & $8(15)$ \\
\hline 31 & $-\mathrm{C} 26$ & - C27 & $=1$ \\
\hline C31 & $-\mathrm{C} 26$ & $-\mathrm{C} 25$ & $=1$ \\
\hline C27 & $-C 26$ & $-C 25$ & $5(17)$ \\
\hline $\mathrm{C} 28$ & $-\mathrm{C} 27$ & $-\mathrm{C} 26$ & 57(19) \\
\hline C28 & $-\mathrm{C} 27$ & $-\mathrm{H} 27$ & $=1$ \\
\hline C26 & $-\mathrm{C} 27$ & $-\mathrm{H} 27$ & \\
\hline 20 & $-\mathrm{C} 28$ & - C27 & $50(2)$ \\
\hline 2 & $-\mathrm{C} 28$ & $-\mathrm{H} 28$ & $=1$ \\
\hline 27 & $-C 28$ & $\mathrm{H} 28$ & $=1$ \\
\hline $\mathrm{C} 28$ & $-C 29$ & $-\mathrm{C} 30$ & $1(19$ \\
\hline C28 & $-C 29$ & $-\mathrm{H} 29$ & $=1$ \\
\hline & $-\mathrm{C} 29$ & $-\mathrm{H} 2 \mathrm{~g}$ & $=1$ \\
\hline C29 & $-\mathrm{C} 30$ & - C31 & $=1$ \\
\hline & - C30 & - H3O & $=1$ \\
\hline & $-\mathrm{C} 30$ & - H3O & $=1$ \\
\hline C30 & $-\mathrm{C} 31$ & $-\mathrm{C} 26$ & $47(19$ \\
\hline C30 & $-\mathrm{C} 31$ & $-\mathrm{H} 31$ & $=119.80$ \\
\hline & - C31 & $-\mathrm{H} 31$ & $=1$ \\
\hline$C 25$ & $-\mathrm{C} 32$ & $-\mathrm{C} 33$ & $=13$ \\
\hline $\mathrm{C} 25$ & $-\mathrm{C} 32$ & $-\mathrm{H} 32$ & 30 \\
\hline C33 & - C32 & $-\mathrm{H} 32$ & $=114.30$ \\
\hline C34 & - C33 & $-\mathrm{C} 38$ & $=117.76(18)$ \\
\hline C34 & - C33 & $-\mathrm{C} 32$ & $=117.37(17)$ \\
\hline C38 & $-\mathrm{C} 33$ & $-\mathrm{C} 32$ & $=124.83(17)$ \\
\hline C33 & - C34 & - C35 & $=121.70(2)$ \\
\hline
\end{tabular}




$\begin{array}{llll}\text { C33 } & -\mathrm{C} 34 & -\mathrm{H} 34 & =119.20 \\ \mathrm{C} 35 & -\mathrm{C} 34 & -\mathrm{H} 34 & =119.20 \\ \mathrm{C} 36 & -\mathrm{C} 35 & -\mathrm{C} 34 & =119.70(2) \\ \mathrm{C} 36 & -\mathrm{C} 35 & -\mathrm{H} 35 & =120.10 \\ \mathrm{C} 34 & -\mathrm{C} 35 & -\mathrm{H} 35 & =120.10 \\ \mathrm{C} 35 & -\mathrm{C} 36 & -\mathrm{C} 37 & =119.57(19) \\ \mathrm{C} 35 & -\mathrm{C} 36 & -\mathrm{H} 36 & =120.20 \\ \mathrm{C} 37 & -\mathrm{C} 36 & -\mathrm{H} 36 & =120.20 \\ \mathrm{C} 36 & -\mathrm{C} 37 & -\mathrm{C} 38 & =120.90(2) \\ \mathrm{C} 36 & -\mathrm{C} 37 & -\mathrm{H} 37 & =119.60 \\ \mathrm{C} 38 & -\mathrm{C} 37 & -\mathrm{H} 37 & =119.60 \\ \mathrm{C} 33 & -\mathrm{C} 38 & -\mathrm{C} 37 & =120.33(19) \\ \mathrm{C} 33 & -\mathrm{C} 38 & -\mathrm{H} 38 & =119.80 \\ \mathrm{C} 37 & -\mathrm{C} 38 & -\mathrm{H} 38 & =119.80\end{array}$

Table S5. Torsion angles [ $\left[^{\circ}\right]$ for 3aa.

$\begin{array}{lllll}\mathrm{C} 13 & -\mathrm{P} 1 & -\mathrm{C} 1 & -\mathrm{C} 6 & =171.49(13) \\ \mathrm{C} 7 & -\mathrm{P} 1 & -\mathrm{C} 1 & -\mathrm{C} 6 & =65.51(15) \\ \mathrm{C} 13 & -\mathrm{P} 1 & -\mathrm{C} 1 & -\mathrm{C} 2 & =-66.54(13) \\ \mathrm{C} 7 & -\mathrm{P} 1 & -\mathrm{C} 1 & -\mathrm{C} 2 & =-172.52(12) \\ \mathrm{C} 6 & -\mathrm{C} 1 & -\mathrm{C} 2 & -\mathrm{C} 3 & =-53.00(2) \\ \mathrm{P} 1 & -\mathrm{C} 1 & -\mathrm{C} 2 & -\mathrm{C} 3 & =-174.79(13) \\ \mathrm{C} 1 & -\mathrm{C} 2 & -\mathrm{C} 3 & -\mathrm{C} 4 & =54.10(2) \\ \mathrm{C} 2 & -\mathrm{C} 3 & -\mathrm{C} 4 & -\mathrm{C} 5 & =-55.20(3) \\ \mathrm{C} 3 & -\mathrm{C} 4 & -\mathrm{C} 5 & -\mathrm{C} 6 & =56.60(3) \\ \mathrm{C} 4 & -\mathrm{C} 5 & -\mathrm{C} 6 & -\mathrm{C} 1 & =-57.00(2) \\ \mathrm{C} 2 & -\mathrm{C} 1 & -\mathrm{C} 6 & -\mathrm{C} 5 & =54.60(2) \\ \mathrm{P} 1 & -\mathrm{C} 1 & -\mathrm{C} 6 & -\mathrm{C} 5 & =176.85(15) \\ \mathrm{C} 13 & -\mathrm{P} 1 & -\mathrm{C} 7 & -\mathrm{C} 8 & =-55.55(15) \\ \mathrm{C} 1 & -\mathrm{P} 1 & -\mathrm{C} 7 & -\mathrm{C} 8 & =50.00(15) \\ \mathrm{C} 13 & -\mathrm{P} 1 & -\mathrm{C} 7 & -\mathrm{C} 12 & =72.46(14) \\ \mathrm{C} 1 & -\mathrm{P} 1 & -\mathrm{C} 7 & -\mathrm{C} 12 & =178.01(12) \\ \mathrm{C} 12 & -\mathrm{C} 7 & -\mathrm{C} 8 & -\mathrm{C} 9 & =58.10(2) \\ \mathrm{P} 1 & -\mathrm{C} 7 & -\mathrm{C} 8 & -\mathrm{C} 9 & =-173.33(13) \\ \mathrm{C} 7 & -\mathrm{C} 8 & -\mathrm{C} 9 & -\mathrm{C} 10 & =-56.80(2) \\ \mathrm{C} 8 & -\mathrm{C} 9 & -\mathrm{C} 10 & -\mathrm{C} 11 & =54.00(2) \\ \mathrm{C} 9 & -\mathrm{C} 10 & -\mathrm{C} 11 & -\mathrm{C} 12 & =-53.40(2) \\ \mathrm{C} 10 & -\mathrm{C} 11 & -\mathrm{C} 12 & -\mathrm{C} 7 & =55.90(2) \\ \mathrm{C} 8 & -\mathrm{C} 7 & -\mathrm{C} 12 & -\mathrm{C} 11 & =-57.90(2) \\ \mathrm{P} 1 & -\mathrm{C} 7 & -\mathrm{C} 12 & -\mathrm{C} 11 & =169.45(13) \\ \mathrm{C} 1 & -\mathrm{P} 1 & -\mathrm{C} 13 & -\mathrm{C} 14 & =-28.45(17) \\ \mathrm{C} 7 & -\mathrm{P} 1 & -\mathrm{C} 13 & -\mathrm{C} 14 & =76.09(16) \\ \mathrm{C} 1 & -\mathrm{P} 1 & -\mathrm{C} 13 & -\mathrm{C} 18 & =152.41(14) \\ \mathrm{C} 7 & -\mathrm{P} 1 & -\mathrm{C} 13 & -\mathrm{C} 18 & =-103.05(15) \\ \mathrm{C} 18 & -\mathrm{C} 13 & -\mathrm{C} 14 & -\mathrm{C} 15 & =0.60(3) \\ \mathrm{P} 1 & -\mathrm{C} 13 & -\mathrm{C} 14 & -\mathrm{C} 15 & =-178.57(15) \\ \mathrm{C} 13 & -\mathrm{C} 14 & -\mathrm{C} 15 & -\mathrm{C} 16 & =0.50(3) \\ \mathrm{C} 14 & -\mathrm{C} 15 & -\mathrm{C} 16 & -\mathrm{C} 17 & =-0.90(3) \\ \mathrm{C} 15 & -\mathrm{C} 16 & -\mathrm{C} 17 & -\mathrm{C} 18 & =0.40(3) \\ \mathrm{C} 16 & -\mathrm{C} 17 & -\mathrm{C} 18 & -\mathrm{C} 13 & =0.70(3) \\ \mathrm{C} 16 & -\mathrm{C} 17 & -\mathrm{C} 18 & -\mathrm{C} 19 & =175.56(18) \\ & & & & \end{array}$




\begin{tabular}{|c|c|c|c|c|}
\hline & $-\mathrm{C} 13$ & - C18 & - C17 & $=-1.10(3)$ \\
\hline 1 & - C13 & - C18 & - C17 & $=178.05(13)$ \\
\hline & - C13 & - C18 & $-\mathrm{C} 19$ & $=-175.68(16)$ \\
\hline & C13 & - C18 & - C19 & $=3.50(2)$ \\
\hline & - C18 & - C19 & $-\mathrm{C} 20$ & $=-65.10(2)$ \\
\hline & - C18 & - C19 & $-\mathrm{C} 20$ & $=109.60(2)$ \\
\hline & - C18 & - C19 & $-\mathrm{C} 24$ & $=113.70(2)$ \\
\hline & - C18 & - C19 & $-\mathrm{C} 24$ & $=-71.70(3)$ \\
\hline & - C19 & - C20 & $-C 21$ & $=1.80(3)$ \\
\hline & - C19 & $-\mathrm{C} 20$ & $-C 21$ & $=-179.30($ \\
\hline & $-\mathrm{C} 20$ & $-C 21$ & $-\mathrm{C} 22$ & $=0.70(3)$ \\
\hline 0 & $-C 21$ & $-\mathrm{C} 22$ & $-\mathrm{C} 23$ & $=-1.90(3)$ \\
\hline 1 & $-\mathrm{C} 22$ & $-\mathrm{C} 23$ & $-\mathrm{C} 24$ & $=0.60(3)$ \\
\hline & $-\mathrm{C} 23$ & $-C 24$ & - C19 & $=1.90$ \\
\hline & $-\mathrm{C} 23$ & $-C 24$ & $-C 25$ & $=-175$. \\
\hline & $-\mathrm{C} 19$ & $-\mathrm{C} 24$ & $-\mathrm{C} 23$ & $=-3.10$ \\
\hline & $-\mathrm{C} 19$ & $-\mathrm{C} 24$ & $-\mathrm{C} 23$ & $=178$. \\
\hline 0 & - C19 & $-\mathrm{C} 24$ & $-\mathrm{C} 25$ & $=174.47$ \\
\hline & - C19 & $-\mathrm{C} 24$ & $-\mathrm{C} 25$ & \\
\hline & $c_{2}$ & & 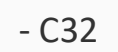 & 50 \\
\hline & $-\mathrm{C} 24$ & $-\mathrm{C} 25$ & - C32 & $=123$. \\
\hline & $-\mathrm{C} 24$ & $-\mathrm{C} 25$ & $-\mathrm{C} 26$ & $=1$ \\
\hline & $-\mathrm{C} 24$ & $-\mathrm{C} 25$ & $-\mathrm{C} 26$ & \\
\hline & $-\mathrm{C} 25$ & - C26 & - C31 & \\
\hline & $-\mathrm{C} 25$ & $-\mathrm{C} 2$ & - C31 & \\
\hline & $-\mathrm{C} 2$ & $-C 26$ & $-C 27$ & $=1$ \\
\hline & $-\mathrm{C} 2$ & $-\mathrm{C} 26$ & $-\mathrm{C} 27$ & $=-59$. \\
\hline & $-\mathrm{C} 26$ & $-C 27$ & $-\mathrm{C} 28$ & $=-0.4$ \\
\hline & $-\mathrm{C} 26$ & $-\mathrm{C} 27$ & $-\mathrm{C} 28$ & - 170 \\
\hline & $-\mathrm{C} 2$ & $-\mathrm{C} 28$ & $-C 29$ & $=-1.00$ \\
\hline & $-\mathrm{C} 2$ & $-C 29$ & $-\mathrm{C} 30$ & $=2.00$ \\
\hline & & & - C31 & $=-1.5$ \\
\hline & $-\mathrm{C} 3 \mathrm{C}$ & C31 & $-\mathrm{C} 26$ & $=0.1$ \\
\hline & $-C 26$ & - C31 & - C30 & $-\quad 08$ \\
\hline & $-\mathrm{C} 26$ & - C31 & - C30 & $=-178.02$ \\
\hline & $-\mathrm{C} 25$ & $-\mathrm{C} 32$ & - C33 & $=174.11$ \\
\hline & & 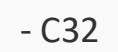 & - C33 & $=-2.90$ \\
\hline & & C3 & - C34 & $=158.3$ \\
\hline & $-\mathrm{C} 3$ & C23 & $-\mathrm{C} 38$ & $=-24.00(3)$ \\
\hline & $-\mathrm{C} 33$ & - C34 & $-\mathrm{C} 35$ & $=1.70(3)$ \\
\hline & - C33 & - C34 & - C35 & $=179.62(1$ \\
\hline & $-C^{3}$ & $-\mathrm{C3}$ & - C36 & $=-1.40(3)$ \\
\hline & & & (3/ & $=0.20(3)$ \\
\hline & $-\mathrm{C} 3$ & $-\mathrm{C3}$ & $-\mathrm{C} 38$ & $=0.60(4)$ \\
\hline & $-\mathrm{C} 3$ & - C38 & - C37 & $=-1.00(3)$ \\
\hline & - C33 & - C38 & - C37 & $=-178.68(1)$ \\
\hline C36 & - C37 & - C38 & - C33 & $=-0.20(3)$ \\
\hline
\end{tabular}




\section{Crystal measurement for 4aa:}

Crystallization by diffusion method from $\mathrm{CH}_{2} \mathrm{Cl}_{2}$ solution with $n$-hexane has afforded single crystal to analyze by X-ray diffraction.

The single crystal X-ray diffraction studies were carried out on D8 VENTURE Bruker AXS diffractometer equipped with a (CMOS) PHOTON 100 detector, $\left[{ }^{*}\right]$, Mo- $K \alpha$ radiation $(\lambda=0.71073$, multilayer monochromator), $T=150 \mathrm{~K}$; triclinic $P-1$ (I.T.\#2), $a=15.0306(13), b=16.0708(13), c=19.4591(16), \alpha$ $=95.629(3), b=105.254(3), \gamma=108.810(3), V=4205.6(6){ }^{3} . Z=2, d=1.183 \mathrm{~g} . \mathrm{cm}^{-3}, \mu=0.164 \mathrm{~mm}^{-1}$. The structure was solved by dual-space algorithm using the SHELXT program [1], and then refined with full-matrix least-squares methods based on $F^{2}$ (SHELXL ) [2]. The contribution of the disordered solvents to the calculated structure factors was estimated following the BYPASS algorithm [3], implemented as the SQUEEZE option in PLATON [4]. A new data set, free of solvent contribution, was then used in the final refinement. All non-hydrogen atoms were refined with anisotropic atomic displacement parameters. $\mathrm{H}$ atoms were finally included in their calculated positions and treated as riding on their parent atom with constrained thermal parameters. A final refinement on $F^{2}$ with 19092 unique intensities and 982 parameters converged at $\omega R_{F}^{2}=0.1172\left(R_{F}=0.0490\right)$ for 13976 observed reflections with $I>2 \sigma(I)$.

[1] G. M. Sheldrick, Acta Cryst. A71 (2015) 3-8

[2] Sheldrick G.M., Acta Cryst. C71 (2015) 3-8

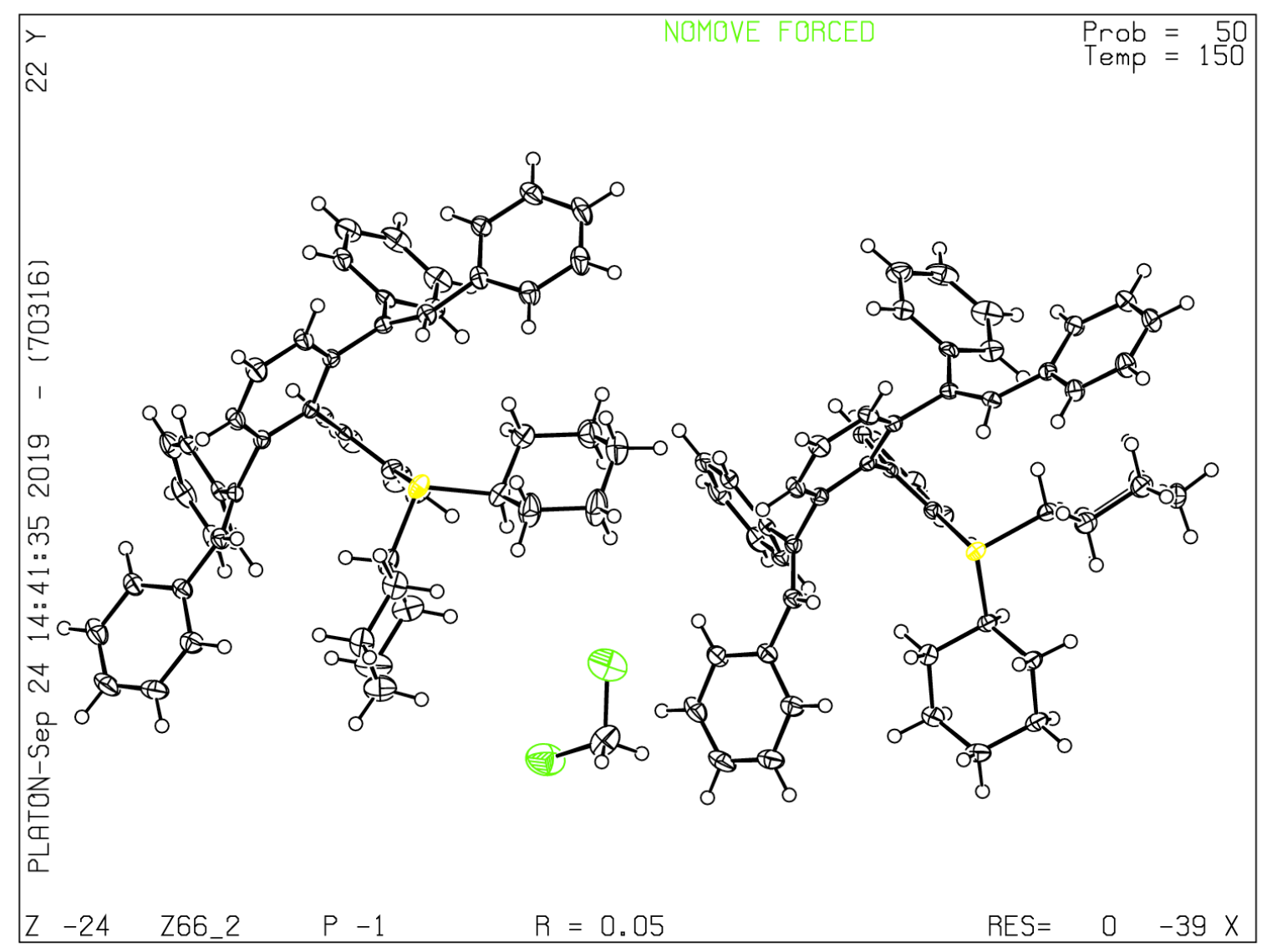

Figure S2. ORTEP diagram of the solid-state molecular structure of 4aa. Thermal ellipsoids are at the 50\% probability level. (CCDC 1968421). 
Table S6. Crystal data and structure refinement for 4aa.

\begin{tabular}{|c|c|}
\hline Empirical formula & $\mathrm{C}_{105} \mathrm{H}_{104} \mathrm{Cl}_{2} \mathrm{P}_{2}$ \\
\hline Formula weight & $1498.72 \mathrm{~g} / \mathrm{mol}$ \\
\hline Temperature & $150 K$ \\
\hline Wavelength & $0.71073 \AA$ \\
\hline Crystal system, space group & triclinic, $P-1$ \\
\hline \multirow[t]{6}{*}{ Unit cell dimensions } & $a=15.0306(13) \AA$ \\
\hline & $b=16.0708(13) \AA$ \\
\hline & $c=19.4591(16) \AA$ \\
\hline & $\alpha=95.629(3)^{\circ}$ \\
\hline & $\beta=105.254(3)^{\circ}$ \\
\hline & $\gamma=108.810(3)^{\circ}$ \\
\hline Volume & $4205.6(6) \AA^{3}$ \\
\hline Z, Calculated density & $2,1.183 \mathrm{~g} \cdot \mathrm{cm}^{-3}$ \\
\hline Absorption coefficient & $0.164 \mathrm{~mm}^{-1}$ \\
\hline$F(000)$ & 1596 \\
\hline Crystal size & $0.580 \times 0.320 \times 0.180 \mathrm{~mm}$ \\
\hline Crystal color & colourless \\
\hline Theta range for data collection & 2.215 to $27.527^{\circ}$ \\
\hline h_min, h_max & $-19,19$ \\
\hline k_min, k_max & $-20,20$ \\
\hline I_min, I_max & $-24,25$ \\
\hline Reflections collected / unique & $79668 / 19092\left[R(\text { int })^{a}=0.0683\right]$ \\
\hline Reflections $[\mid>2 \sigma]$ & 13976 \\
\hline Completeness to theta_max & 0.986 \\
\hline Absorption correction type & multi-scan \\
\hline Max. and min. transmission & $0.971,0.874$ \\
\hline Refinement method & Full-matrix least-squares on $F^{2}$ \\
\hline Data / restraints / parameters & 19092 / 0 / 982 \\
\hline bS (Goodness-of-fit) & 1.011 \\
\hline Final $R$ indices $[\mid>2 \sigma]$ & $R 1^{c}=0.0490, w R 2^{d}=0.1172$ \\
\hline$R$ indices (all data) & $R 1^{c}=0.0779, w R 2^{d}=0.1339$ \\
\hline Largest diff. peak and hole & 1.058 and $-0.545 \mathrm{e}^{-} . \AA^{-3}$ \\
\hline
\end{tabular}

Table S7. Fractional atomic coordinates, site occupancy (\%) and equivalent isotropic displacement parameters $\left(\AA^{2}\right)$ for 4 aa. $U(e q)$ is defined as one third of the trace of the orthogonalized Uij tensor.

\begin{tabular}{|c|c|c|c|c|c|}
\hline tom & $\mathrm{x}$ & y & z & occ. & $\mathrm{U}(\mathrm{eq}) \quad \mathrm{adp}$ \\
\hline P1 & $0.16644(4)$ & $0.83103(3)$ & $0.29726(3)$ & 1 & 0.02289(11) Uani \\
\hline $\mathrm{C} 1$ & $0.22780(15)$ & $0.74712(13)$ & 3) $0.29264(11)$ & 1 & $0.0262(4)$ Uani \\
\hline $\mathrm{H} 1$ & 0.181477 & 0.686630 & 0.293241 & 1 & 0.031 Uiso \\
\hline C2 & $0.25363(16)$ & ) $0.74405(14)$ & $0.22142(11)$ & 1 & $0.0307(4)$ Uani \\
\hline $\mathrm{H} 2 \mathrm{~A}$ & 0.191570 & 0.718118 & 0.180057 & 1 & 0.037 Uiso \\
\hline $\mathrm{H} 2 \mathrm{~B}$ & 0.288433 & 0.806094 & 0.216946 & 1 & 0.037 \\
\hline C3 & $0.31849(17)$ & ) $0.68908(16)$ & 5) $0.21650(13)$ & ) 1 & 0.0383(5) Uani \\
\hline H3A & 0.280487 & 0.625178 & 0.213939 & 1 & $0.046 \quad$ Uiso \\
\hline H3B & 0.336908 & 0.693747 & 0.171392 & 1 & $0.046 \quad$ Uiso \\
\hline C4 & $0.41182(17)$ & $0.72128(17)$ & 7) $0.28156(14)$ & ) 1 & $0.0429(6) \quad$ Uani \\
\hline $\mathrm{H} 4 \mathrm{~A}$ & 0.450804 & 0.682700 & 0.277954 & 1 & $0.051 \quad$ Uiso \\
\hline $\mathrm{H} 4 \mathrm{~B}$ & 0.452988 & 0.783511 & 0.281956 & 1 & 0.051 \\
\hline C5 & $0.3850(2)$ & $0.71800(19)$ & $0.35109(14)$ & 1 & 0.0497(7) Uani \\
\hline
\end{tabular}




\begin{tabular}{|c|c|c|c|c|c|}
\hline $5 \mathrm{~A}$ & & 0.740331 & 0.393188 & 1 & 0.060 \\
\hline 5B & 7711 & 0.655055 & 0.351940 & 1 & Uiso \\
\hline & 10(18) & $0.77501(18)$ & $0.35782(13)$ & 1 & $0.0434(6)$ Uani \\
\hline & 0.362072 & 0.838837 & 0.362003 & 1 & $0.052 \quad$ Uiso \\
\hline$B$ & 0.303609 & 0.769278 & 0.402827 & 1 & 0.052 \\
\hline & 0.1049 & $0.81011(12)$ & $0.36880(10)$ & 1 & $0.0255(4)$ Uani \\
\hline & 0.056149 & $0.841031 \quad 0$ & $0.359795 \quad 1$ & 1 & $0.031 \quad$ Uiso \\
\hline & 0.0447 & $0.71340(14)$ & $0.36904(12)$ & 1 & 0.0364(5) Uani \\
\hline & $0.08 \mathrm{~s}$ & 0.679434 & 0.380267 & 1 & $0.044 \quad$ Uiso \\
\hline$B$ & -0.00 & 0.684625 & 0.320189 & 1 & 0.044 \\
\hline & -0.010 & $0.71002(17)$ & $0.42543(12)$ & 1 & 0.0459(6) Uani \\
\hline & -0.05 & 0.739874 & 0.412027 & 1 & 0.055 Uiso \\
\hline & -0.04 & 0.646700 & 0.425623 & 1 & 0.055 Uiso \\
\hline & 0.061 & ) $0.75627(16)$ & $0.50105(12)$ & ) 1 & $0.0429(6)$ Uan \\
\hline $.0 A$ & 0.0 & 0.756143 & 0.535967 & 1 & $0.051 \quad$ Uiso \\
\hline. $\mathrm{OB}$ & 0.10 & 0.722623 & 0.516520 & 1 & 0.051 \\
\hline & 0.12 & ) $0.85227(14)$ & $0.50210(11)$ & ) 1 & 0.0344(5) Uani \\
\hline $1 \mathrm{~A}$ & 0.17 & 0.879135 & 0.550868 & 1 & 0.041 Uiso \\
\hline $11 B$ & 0.08 & 0.887779 & 0.492773 & 1 & 0.041 \\
\hline 2 & 0.177 & ) $0.85686(14)$ & ) $0.44508(11)$ & ) 1 & $0.0315(5) \quad \mathrm{Ua}$ \\
\hline $2 \mathrm{~A}$ & 0.22 & 0.828003 & 0.458005 & 1 & $0.038 \quad$ Uiso \\
\hline $2 \mathrm{~B}$ & 0.21 & 0.920479 & 0.444958 & 1 & $0.038 \quad$ Uiso \\
\hline & 0.05 & ) $0.78358(11)$ & $0.21542(10)$ & ) 1 & 0.0201(4) Uani \\
\hline 4 & 0.007 & ) 0.69097(12) & $0.18858(11)$ & 1 & 0.0294(4) Uani \\
\hline 14 & 16 & $0.650722 \quad 0$ & 0.212683 & 1 & 0.035 Uiso \\
\hline 5 & -0.07 & $0.65668(13)$ & $0.12787(11)$ & 1 & $0.0328(5)$ Uan \\
\hline 5 & -0.10 & 0.593646 & 0.111996 & 1 & 0.039 Uiso \\
\hline 6 & -0.11 & ) $0.71463(13)$ & $0.09041(10)$ & 1 & $0.0254(4)$ Uan \\
\hline 16 & -0.16 & $0.691578 \quad 0$ & 0.048121 & 1 & $0.031 \quad$ Uiso \\
\hline 17 & -0.06 & $0.80618(12)$ & $0.11482(10)$ & 1 & $0.0198(4) \mathrm{Ua}$ \\
\hline 17 & -0.08 & $0.845737 \quad 0$ & 0.088128 & 1 & 0.024 \\
\hline & 0.01 & ) $0.84170(11)$ & ) 0.17806(9) & 1 & 0.0153(3) Uani \\
\hline & 0.06 & ) $0.94133(11)$ & ) $0.20577(9)$ & 1 & $0.0154(3)$ Uani \\
\hline & 0.02 & ) 0.98397(11) & $0.25241(9)$ & 1 & $0.0167(3)$ Uani \\
\hline 1 & 0.07 & ) $1.07622(12)$ & ) $0.28155(10)$ & 1 & $0.0227(4)$ Uani \\
\hline 21 & 20 & $1.105210 \quad 0$ & 0.312998 & 1 & $0.027 \quad$ Uiso \\
\hline $\mathrm{C} 22$ & 0.158 & ) $1.12562(12)$ & $0.26538(11)$ & 1 & $0.0271(4)$ Uani \\
\hline 22 & 0.19 & $1.187743 \quad 0$ & 0.286244 & 1 & 0.033 \\
\hline & 0.19 & ) $1.08413(12)$ & $0.21860(11)$ & 1 & 0.0230(4) Uani \\
\hline & 0.25 & $1.118467 \quad 0$ & 0.206944 & 1 & 0.028 \\
\hline 24 & $(12)$ & ) $0.99275(11)$ & ) 0.18836(9 & 1 & 0.0169(3) Uani \\
\hline & -0.0 & ) $0.93805(11)$ & 0.27362( & & $0.0176(3)$ Uan \\
\hline C26 & -0.157 & ) $0.88287(11)$ & $0.21563(10)$ & 1 & $0.0179(3)$ Uani \\
\hline $\mathrm{C} 27$ & $-0.19199(13)$ & ) $0.91584(13)$ & $0.15501(10)$ & 1 & $0.0227(4)$ Uan \\
\hline-127 & -0.15 & $0.972896 \quad 0$ & 0.148881 & 1 & $0.027 \quad$ Uiso \\
\hline $\mathrm{C} 28$ & -0.28 & $0.86565(15)$ & ) 0.10319(1 & 1 & 0.0318(5) Uan \\
\hline $\mathrm{H} 28$ & -0.305 & $0.888575 \quad 0$ & 0.061722 & 1 & $0.038 \quad$ Uiso \\
\hline C29 & $-0.33901(14)$ & $0.78288(15)$ & $0.11152(12)$ & 1 & $0.0344(5)$ Uani \\
\hline $\mathrm{H} 29$ & -0.401397 & $0.749400 \quad 0$ & 0.076300 & 1 & 0.041 \\
\hline C30 & $-0.30463(15)$ & ) $0.74877(14)$ & ) $0.17108(12)$ & 1 & 0.0317(5) Uan \\
\hline & -0.34 & $0.691580 \quad 0$ & 0.176744 & 1 & $0.038 \quad$ Uiso \\
\hline 31 & $-0.21376(14)$ & ) $0.79800(12)$ & $0.22272(11)$ & & 0.0238(4) Uani \\
\hline
\end{tabular}




\begin{tabular}{|c|c|c|c|c|}
\hline 31 & & $0.773818 \quad 0.263227$ & 1 & 0.029 Uiso \\
\hline 32 & $-0.06050(13)$ & $0.95411(12) 0.34358(10)$ & 1 & $0.0205(4)$ Uan \\
\hline 32 & 0.003657 & 0.9826300 .377933 & 1 & $0.025 \quad$ Uiso \\
\hline & $-0.14343(13)$ & ) $0.93352(12) 0.37431(10)$ & 1 & $0.0212(4)$ Uan \\
\hline 34 & $-0.23604(14)$ & ) $0.93572(12) 0.33731(11)$ & 1 & 0.0247(4) Uani \\
\hline 34 & -0.248433 & 0.946880 & 1 & 0.030 \\
\hline 35 & $-0.30968(15)$ & ) $0.92177(14) 0.37008(12)$ & 1 & $0.0326(5)$ Uani \\
\hline 35 & -0.372307 & $0.922791 \quad 0.344153$ & 1 & $0.039 \quad$ Uiso \\
\hline & $-0.29269(17)$ & ) $0.90638(14) 0.44027(13)$ & 1 & $0.0358(5)$ Uan \\
\hline 6 & -0.343403 & $0.896911 \quad 0.462485$ & 1 & $0.043 \quad$ Uiso \\
\hline 37 & $-0.20180(17)$ & ) $0.90486(13) 0.47777(12)$ & 1 & $0.0331(5)$ Uani \\
\hline 37 & -0.189784 & $0.894540 \quad 0.526027$ & 1 & 0.040 \\
\hline 8 & $-0.12780(15)$ & $0.91837(13) 0.44529(11)$ & 1 & $0.0268(4)$ Uan \\
\hline 38 & -0.065421 & $0.917291 \quad 0.471745$ & 1 & $0.032 \quad$ Uiso \\
\hline 39 & 0.19 & $0.95313(11) 0.14154(10)$ & 1 & 0.0180(3) Uani \\
\hline 10 & 0.13 & $0.90457(12) 0.0$ & & $0.0187(4)$ \\
\hline 1 & (14) & $0.94722(14) 0.0$ & 1 & $0.0279(4)$ Uani \\
\hline 1 & 0.09 & $1.005251 \quad 0.031$ & 1 & 0.033 \\
\hline 12 & $0.04500(15)$ & $0.90548(16)-0.05$ & 1 & $0.0350(5) \mathrm{Ua}$ \\
\hline 42 & 0.01 & $0.935143-0.09$ & 1 & $0.042 \quad$ Uiso \\
\hline 3 & 0.04 & $131(16)-0$ & 1 & 0.0354(5) Uani \\
\hline 43 & 0.01 & $0.793343-0.13$ & 1 & 0.043 \\
\hline 4 & 7(15) & $0.77809(14)-0$. & 1 & $0.0324(5) \quad \mathrm{Ua}$ \\
\hline 14 & 0.08 & $0.719800-0.04$ & 1 & $0.039 \quad$ Uiso \\
\hline 45 & 0.13 & $0.81887(12) 0.0$ & 1 & 0.0255(4) Uani \\
\hline 45 & 6 & $0.787996 \quad 0.07$ & 1 & 0.031 Uiso \\
\hline 6 & $0(13)$ & $0.96843(11) 0.1$ & 1 & $0.0202(4)$ Uani \\
\hline 6 & 0.32 & $0.997308 \quad 0.217$ & 1 & 0.024 \\
\hline 7 & 0.357 & $0.94683(11) 0.1$ & 1 & $0.0216(4) \quad U a$ \\
\hline 18 & 0.34 & $0.94524(12) 0.0$ & 1 & $0.0241(4) \quad \mathrm{Ua}$ \\
\hline 448 & 77 & $0.959112 \quad 0.028$ & 1 & $0.029 \quad$ Uiso \\
\hline C49 & 0.41 & $0.92379(14) 0.0$ & 1 & 0.0307(4) Uani \\
\hline 9 & $9 \quad \mathrm{c}$ & $668-0.02$ & 1 & $0.037 \quad$ Uiso \\
\hline & 0.48 & $0.90394(14) 0.06$ & 1 & 0.0332(5) Uani \\
\hline & 0.530 & 0.887860 & 1 & $0.040 \quad$ Uiso \\
\hline 1 & 0.50 & $0.90748(14) 0.1$ & 1 & $0.0317(5) \quad \mathrm{Ua}$ \\
\hline 51 & 1 & $0.894907 \quad 0.16$ & 1 & $0.038 \quad$ Uiso \\
\hline 52 & $0.43849(13)$ & $0.92945(13) 0.172$ & 1 & 0.0262(4) Uani \\
\hline & & $0.932751 \quad 0.223$ & 1 & $0.031 \quad$ Uiso \\
\hline 2 & 0.622 & $0.27673(3) \quad 0.29955($ & 1 & $0.01755(10)$ Uani \\
\hline & $2(13)$ & $0.17549(11) 0.326$ & 1 & 0.0191(4) Uani \\
\hline & & $320 \quad 0.28$ & 1 & 0.023 \\
\hline & 0.61 & $0.14949(13) 0.3$ & 1 & $0.0267(4) \quad$ Uani \\
\hline H54A & & $0.131385 \quad 0.371$ & 1 & Uiso \\
\hline 54B & & $0.202181 \quad 0.429545$ & 1 & 0.032 Uiso \\
\hline & $3(16)$ & $0.07226(14) 0.416$ & 1 & $0.0316(5) \quad \mathrm{Ua}$ \\
\hline & & $0.017991 \quad 0.37$ & 1 & $0.038 \quad$ Uiso \\
\hline H55B & & 0.058626 & 1 & 0.038 \\
\hline C56 & $0.47453(16)$ & $0.09517(15) \quad 0.44184(11)$ & 1 & $0.0325(5) \quad \mathrm{Ua}$ \\
\hline & & 0.145811 & 1 & 0.039 \\
\hline & & 0.042855 & 1 & 0.039 \\
\hline 57 & $0.40746(15)$ & $0.12061(13) 0.38086(11)$ & 1 & $0.0274(4) \mathrm{Ua}$ \\
\hline
\end{tabular}




\begin{tabular}{|c|c|c|c|c|c|}
\hline 57A & & 0.139809 & 0.399419 & 1 & Uiso \\
\hline 57B & 0.368715 & 0.067486 & 0.341059 & 1 & Uiso \\
\hline 8 & $0.46757(14)$ & $0.19644(13)$ & $0.35122(11)$ & 1 & $0.0242(4) \quad$ Uan \\
\hline $8 \mathrm{~A}$ & 0.498582 & 0.251823 & 0.389310 & 1 & 0.029 Uiso \\
\hline $58 \mathrm{~B}$ & 0.422293 & 0.207921 & 0.309331 & 1 & 0.029 \\
\hline 9 & $0.70840(13)$ & $0.24040(12)$ & ) $0.25994(10)$ & 1 & $0.0203(4) \quad$ Uani \\
\hline 59 & 0.722914 & $0.280160 \quad 0$ & 0.224732 & 1 & $0.024 \quad$ Uiso \\
\hline 0 & $0.66980(14)$ & $0.14414(13)$ & ) $0.21606(11)$ & 1 & $0.0277(4)$ Uan \\
\hline $50 \mathrm{~A}$ & 0.6528 & 0.101264 & 0.248001 & 1 & 0.033 Uiso \\
\hline $50 \mathrm{~B}$ & 0.608676 & 0.134148 & 0.176039 & 1 & 0.033 \\
\hline 51 & $0.74605(16)$ & $0.12607(14)$ & ) $0.18429(12)$ & 1 & $0.0335(5) \quad$ Uani \\
\hline $51 \mathrm{~A}$ & 0.719937 & 0.062544 & 0.158976 & 1 & $0.040 \quad$ Uiso \\
\hline $1 \mathrm{~B}$ & 0.75 & 0.164130 & 0.148086 & 1 & 0.040 \\
\hline 52 & $0.84400(15)$ & $0.14558(13)$ & ) $0.24333(12)$ & 1 & $0.0317(5)$ Uan \\
\hline $52 \mathrm{~A}$ & 0.83 & 0.103549 & 0.276971 & 1 & $0.038 \quad$ Uiso \\
\hline $62 B$ & 0.89 & 0.136169 & 0.220794 & 1 & 0.038 \\
\hline 3 & $0.88373(14)$ & $0.24142(13)$ & ) $0.28559(12)$ & 1 & $0.0284(4) \quad$ Uani \\
\hline $3 \mathrm{~A}$ & 0.900182 & 0.283299 & 0.252840 & 1 & 0.034 \\
\hline $63 B$ & 0.94 & 0.251591 & 0.325216 & 1 & 0.034 \\
\hline 54 & $0.80864(13)$ & $0.26118(13)$ & ) 0.31803(11) & 1 & $0.0247(4) \quad$ Uani \\
\hline $64 \mathrm{~A}$ & 0.83 & 0.325218 & 0.342134 & 1 & 0.030 \\
\hline $54 B$ & 0.798739 & 0.224714 & 0.35 & 1 & $0.030 \quad$ Uiso \\
\hline 5 & 0.5333 & $0.28073(11)$ & ) $0.21561(9)$ & 1 & $0.0164(3)$ Uani \\
\hline 66 & $0.53454(12)$ & $0.36374(11)$ & $0.19749(9)$ & 1 & $0.0141(3)$ Uani \\
\hline 67 & 0.4733 & $0.36503(12)$ & ) 0.12975(9) & 1 & $0.0185(4) \quad$ Uani \\
\hline 167 & 0.477 & 0.421077 & 0.116390 & 1 & 0.022 \\
\hline 68 & $0.40748(13)$ & $0.28679(13)$ & ) $0.08191(10)$ & 1 & $0.0228(4)$ Uani \\
\hline 68 & 0.366 & 0.289039 & 0.036352 & 1 & 0.027 \\
\hline C69 & $0.40228(14)$ & $0.20501(12)$ & ) $0.10121(10)$ & 1 & $0.0243(4) \quad$ Uani \\
\hline-169 & 0.355 & $0.150845 \quad 0$ & 0.069612 & 1 & $0.029 \quad$ Uiso \\
\hline C70 & $0.46488(13)$ & $0.20226(12)$ & ) $0.16624(10)$ & 1 & 0.0219(4) Uani \\
\hline-170 & 0.46 & $0.145620 \quad 0$ & 0.178060 & 1 & $0.026 \quad$ Uiso \\
\hline 1 & 0.59 & $0.45366(11)$ & ) $0.24788(9)$ & 1 & 0.0149(3) Uani \\
\hline C72 & 0.689 & $0.50480(11)$ & ) $0.24919(9)$ & 1 & $0.0164(3)$ Uani \\
\hline$C 73$ & 0.7379 & $0.59134(12)$ & ) $0.29230(10)$ & 1 & 0.0203(4) Uani \\
\hline-173 & 0.802580 & $0.625846 \quad 0$ & 0.292504 & 1 & $0.024 \quad$ Uiso \\
\hline C74 & $0.69347(14)$ & $0.62753(12)$ & ) $0.33442(10)$ & 1 & 0.0233(4) Uani \\
\hline H74 & 0.726251 & 0.686965 & 0.362215 & 1 & 0.028 \\
\hline C75 & $0.60032(13)$ & $0.57599(12)$ & ) $0.33564(10)$ & 1 & 0.0211(4) Uani \\
\hline 475 & 0.57018 & $0.599847 \quad 0$ & 0.365727 & 1 & $0.025 \quad$ Uiso \\
\hline C76 & $0.55000(12)$ & $0.48944(11)$ & ) $0.29330(9)$ & 1 & $0.0174(3)$ Uani \\
\hline 77 & 0.452 & $0.43680(11)$ & ) $0.30123(9)$ & 1 & (3) Uani \\
\hline $\mathrm{C78}$ & $0.36409(12)$ & $0.40755(11)$ & ) 0.23513(9) & 1 & $0.0174(3)$ Uani \\
\hline C79 & $0.29874(13)$ & $0.31888(12)$ & ) $0.21388(10)$ & 1 & 0.0209(4) Uani \\
\hline $\mathrm{H} 79$ & 0.310012 & $0.276358 \quad 0$ & 0.242361 & 1 & $0.025 \quad$ Uiso \\
\hline $\mathrm{C} 80$ & $0.21751(14)$ & $0.29170(13)$ & ) $0.15183(11)$ & 1 & 0.0269(4) Uani \\
\hline $\mathrm{H} 80$ & 0.173 & $0.230968 \quad 0$ & 0.138118 & 1 & $0.032 \quad$ Uiso \\
\hline C81 & $0.20068(14)$ & $0.35312(15)$ & ) $0.10974(11)$ & 1 & 0.0295(4) Uani \\
\hline H81 & 0.144611 & $0.334756 \quad 0$ & 0.067413 & 1 & 0.035 Uiso \\
\hline C82 & 0.265 & $0.44106(15)$ & ) $0.12950(11)$ & 1 & $0.0287(4) \quad$ Uani \\
\hline & 0.25 & $0.483077 \quad 0$ & 0.100474 & 1 & $0.034 \quad$ Uiso \\
\hline C83 & $0.34754(14)$ & $0.46857(12)$ & ) $0.19162(10)$ & & 0.0224(4) Uan \\
\hline
\end{tabular}




\begin{tabular}{|c|c|c|c|c|c|}
\hline H83 & 0.392375 & $0.529046 \quad 0$ & 0.204484 & 1 & $0.027 \quad$ Uiso \\
\hline C84 & $0.45086(13)$ & $0.42274(12)$ & ) $0.36808(10)$ & 1 & 0.0203(4) Uani \\
\hline H84 & 0.514179 & $0.443761 \quad 0$ & $0.403852 \quad 1$ & 1 & $0.024 \quad$ Uiso \\
\hline C85 & $0.36767(13)$ & $0.38024(12)$ & ) 0.39521(10) & 1 & 0.0208(4) Uani \\
\hline C86 & $0.38664(15)$ & $0.34175(13)$ & ) $0.45606(10)$ & 1 & 0.0257(4) Uani \\
\hline H86 & 0.451904 & $0.344006 \quad 0$ & $0.478472 \quad 1$ & 1 & 0.031 Uiso \\
\hline C87 & $0.31215(16)$ & $0.30029(13)$ & ) $0.48443(11)$ & 1 & $0.0311(5)$ Uani \\
\hline H87 & 0.326216 & $0.273122 \quad 0$ & $0.525150 \quad 1$ & 1 & 0.037 Uiso \\
\hline $\mathrm{C} 88$ & $0.21712(16)$ & ) $0.29843(14)$ & ) $0.45346(12)$ & 1 & 0.0330(5) Uani \\
\hline H88 & 0.165811 & $0.269618 \quad 0$ & $0.472598 \quad 1$ & 1 & $0.040 \quad$ Uiso \\
\hline C89 & $0.19744(15)$ & $0.33862(13)$ & ) $0.39471(12)$ & 1 & 0.0306(5) Uani \\
\hline H89 & 0.132606 & $0.338278 \quad 0$ & $0.373818 \quad 1$ & 1 & $0.037 \quad$ Uiso \\
\hline C90 & $0.27209(14)$ & ) $0.37965(13)$ & ) 0.36592(11) & 1 & 0.0254(4) Uani \\
\hline H9O & 0.257833 & $0.407660 \quad 0$ & $0.325752 \quad 1$ & 1 & $0.030 \quad$ Uiso \\
\hline C91 & $0.74812(12)$ & ) $0.46987(11)$ & ) $0.21002(9)$ & 1 & 0.0169(3) Uani \\
\hline C92 & $0.70665(12)$ & ) $0.44399(12)$ & ) $0.12922(10)$ & 1 & $0.0183(4)$ Uani \\
\hline C93 & $0.67510(15)$ & ) $0.50338(14)$ & ) $0.09049(11)$ & 1 & 0.0274(4) Uani \\
\hline H93 & 0.683109 & $0.560225 \quad 0$ & 0.115874 & 1 & 0.033 Uiso \\
\hline C94 & $0.63236(16)$ & $0.48082(16)$ & ) $0.01574(12)$ & 1 & 0.0363(5) Uani \\
\hline H94 & 0.611380 & $0.522081-0$ & 0.009769 & 1 & $0.044 \quad$ Uiso \\
\hline C95 & $0.62018(16)$ & ) $0.39830(16)$ & ) $-0.02179(11)$ & 1 & 0.0377(5) Uani \\
\hline H95 & 0.590916 & $0.382744-0$ & 0.073116 & 1 & $0.045 \quad$ Uiso \\
\hline C96 & $0.65050(16)$ & $0.33886(15)$ & ) $0.01530(11)$ & 1 & 0.0359(5) Uani \\
\hline H96 & 0.641756 & $0.281996-0$ & 0.010505 & 1 & $0.043 \quad$ Uiso \\
\hline C97 & $0.69391(14)$ & $0.36127(13)$ & ) 0.09039(10) & 1 & 0.0263(4) Uani \\
\hline H97 & 0.715057 & $0.319749 \quad 0$ & $0.115409 \quad 1$ & 1 & 0.032 Uiso \\
\hline C98 & $0.83623(12)$ & ) $0.47338(11)$ & ) $0.25361(10)$ & 1 & 0.0178(3) Uani \\
\hline H98 & 0.842243 & $0.485049 \quad 0$ & $0.303761 \quad 1$ & 1 & $0.021 \quad$ Uiso \\
\hline C99 & $0.92468(12)$ & ) $0.46330(11)$ & ) $0.24121(10)$ & 1 & 0.0175(3) Uani \\
\hline C100 & $0.93737(13)$ & 3) $0.44026(12)$ & 2) $0.17409(10)$ & & 0.0209(4) Uani \\
\hline H100 & 0.883742 & 0.425704 & 0.130543 & 1 & $0.025 \quad$ Uiso \\
\hline C101 & $1.02785(14)$ & t) $0.43861(13)$ & 3) $0.17093(11)$ & & 0.0253(4) Uani \\
\hline H101 & 1.035355 & 0.422214 & 0.125124 & 1 & $0.030 \quad$ Uiso \\
\hline C102 & $1.10734(14)$ & t) $0.46046(13)$ & 3) $0.23343(11)$ & & 0.0267(4) Uani \\
\hline H102 & 1.169100 & 0.459409 & 0.230541 & 1 & $0.032 \quad$ Uiso \\
\hline C103 & $1.09635(14)$ & t) $0.48389(13)$ & 3) $0.30029(11)$ & & 0.0261(4) Uani \\
\hline H103 & 1.150766 & 0.499510 & 0.343469 & 1 & $0.031 \quad$ Uiso \\
\hline C104 & $1.00631(13)$ & 3) $0.48446(12)$ & 2) $0.30395(10)$ & & 0.0215(4) Uani \\
\hline H104 & 0.999205 & 0.499568 & 0.350139 & 1 & $0.026 \quad$ Uiso \\
\hline $\mathrm{Cl} 1$ & $0.12663(5)$ & $0.51763(6) \quad 0$ & $0.51564(4)$ & 1 & $0.0669(2)$ Uani \\
\hline $\mathrm{Cl} 2$ & $0.31188(5)$ & $0.59780(5) \quad 0$ & $0.48622(4)$ & 1 & 0.05756(18) Uani \\
\hline C105 & $0.25238(18)$ & 3) $0.53179(15)$ & 5) $0.53956(13)$ & & 0.0430(6) Uani \\
\hline $\mathrm{H} 10 \mathrm{C}$ & 0.258706 & 0.472315 & 0.532885 & 1 & $0.052 \quad$ Uiso \\
\hline H10D & 0.285038 & 0.560912 & 0.591522 & 1 & 0.052 \\
\hline
\end{tabular}

Table S8. Bond lengths $[\AA \AA]$ for 4 aa.

$$
\begin{array}{ll}
\mathrm{P} 1-\mathrm{C} 13 & =1.8534(18) \\
\mathrm{P} 1-\mathrm{C} 7 & =1.864(2) \\
\mathrm{P} 1-\mathrm{C} 1 & =1.8705(19) \\
\mathrm{C} 1-\mathrm{C} 6 & =1.533(3)
\end{array}
$$




$$
\begin{aligned}
& \mathrm{C} 1-\mathrm{C} 2=1.535(3) \\
& \mathrm{C} 1-\mathrm{H} 1=1.0000 \\
& \mathrm{C} 2-\mathrm{C} 3=1.526(3) \\
& \mathrm{C} 2-\mathrm{H} 2 \mathrm{~A}=0.9900 \\
& \mathrm{C} 2-\mathrm{H} 2 \mathrm{~B}=0.9900 \\
& \mathrm{C} 3-\mathrm{C} 4=1.519(3) \\
& \mathrm{C} 3-\mathrm{H} 3 \mathrm{~A}=0.9900 \\
& \mathrm{C} 3-\mathrm{H} 3 \mathrm{~B}=0.9900 \\
& \mathrm{C} 4-\mathrm{C} 5=1.511(4) \\
& \mathrm{C} 4-\mathrm{H} 4 \mathrm{~A}=0.9900 \\
& \mathrm{C} 4-\mathrm{H} 4 \mathrm{~B}=0.9900 \\
& \mathrm{C} 5-\mathrm{C} 6=1.533(3) \\
& \mathrm{C} 5-\mathrm{H} 5 \mathrm{~A}=0.9900 \\
& \mathrm{C} 5-\mathrm{H} 5 \mathrm{~B}=0.9900 \\
& \mathrm{C} 6-\mathrm{H} 6 \mathrm{~A}=0.9900 \\
& \mathrm{C} 6-\mathrm{H} 6 \mathrm{~B}=0.9900 \\
& \mathrm{C} 7-\mathrm{C} 8=1.527(3) \\
& \mathrm{C7}-\mathrm{C} 12=1.534(3) \\
& \mathrm{C} 7-\mathrm{H} 7=1.0000 \\
& \mathrm{C} 8-\mathrm{C} 9=1.531(3) \\
& \mathrm{C} 8-\mathrm{H} 8 \mathrm{~A}=0.9900 \\
& \mathrm{C} 8-\mathrm{H} 8 \mathrm{~B}=0.9900 \\
& \mathrm{C} 9-\mathrm{C} 10=1.522(3) \\
& \mathrm{C} 9-\mathrm{H} 9 \mathrm{~A}=0.9900 \\
& \mathrm{C} 9-\mathrm{H9B}=0.9900 \\
& \mathrm{C} 10-\mathrm{C} 11=1.518(3) \\
& \mathrm{C} 10-\mathrm{H} 10 \mathrm{~A}=0.9900 \\
& \mathrm{C} 10-\mathrm{H} 10 \mathrm{~B}=0.9900 \\
& \mathrm{C} 11-\mathrm{C} 12=1.525(3) \\
& \mathrm{C} 11-\mathrm{H} 11 \mathrm{~A}=0.9900 \\
& \mathrm{C} 11-\mathrm{H} 11 \mathrm{~B}=0.9900 \\
& \mathrm{C} 12-\mathrm{H} 12 \mathrm{~A}=0.9900 \\
& \mathrm{C} 12-\mathrm{H} 12 \mathrm{~B}=0.9900 \\
& \mathrm{C} 13-\mathrm{C} 14=1.402(2) \\
& \mathrm{C} 13-\mathrm{C} 18=1.404(2) \\
& \mathrm{C} 14-\mathrm{C} 15=1.384(3) \\
& \mathrm{C} 14-\mathrm{H} 14=0.9500 \\
& \mathrm{C} 15-\mathrm{C} 16=1.383(3) \\
& \mathrm{C} 15-\mathrm{H} 15=0.9500 \\
& \mathrm{C} 16-\mathrm{C} 17=1.381(3) \\
& \mathrm{C} 16-\mathrm{H} 16=0.9500 \\
& \mathrm{C} 17-\mathrm{C} 18=1.398(2) \\
& \mathrm{C} 17-\mathrm{H} 17=0.9500 \\
& \mathrm{C} 18-\mathrm{C} 19=1.501(2) \\
& \mathrm{C} 19-\mathrm{C} 24=1.413(2) \\
& \mathrm{C} 19-\mathrm{C} 20=1.414(2) \\
& \mathrm{C} 20-\mathrm{C} 21=1.405(2) \\
& \mathrm{C} 20-\mathrm{C} 25=1.495(2) \\
& \mathrm{C} 21-\mathrm{C} 22=1.382(3) \\
& \mathrm{C} 21-\mathrm{H} 21=0.9500 \\
& \mathrm{C} 22-\mathrm{C} 23=1.385(3) \\
& \mathrm{C} 22-\mathrm{H} 22=0.9500
\end{aligned}
$$




$$
\begin{aligned}
& \mathrm{C} 23-\mathrm{C} 24=1.394(2) \\
& \mathrm{C} 23-\mathrm{H} 23=0.9500 \\
& \mathrm{C} 24-\mathrm{C} 39=1.496(2) \\
& \mathrm{C} 25-\mathrm{C} 32=1.348(3) \\
& \mathrm{C} 25-\mathrm{C} 26=1.487(2) \\
& \mathrm{C} 26-\mathrm{C} 27=1.387(3) \\
& \mathrm{C} 26-\mathrm{C} 31=1.398(2) \\
& \mathrm{C} 27-\mathrm{C} 28=1.390(3) \\
& \mathrm{C} 27-\mathrm{H} 27=0.9500 \\
& \mathrm{C} 28-\mathrm{C} 29=1.379(3) \\
& \mathrm{C} 28-\mathrm{H} 28=0.9500 \\
& \mathrm{C} 29-\mathrm{C} 30=1.379(3) \\
& \mathrm{C} 29-\mathrm{H} 29=0.9500 \\
& \mathrm{C} 30-\mathrm{C} 31=1.388(3) \\
& \mathrm{C} 30-\mathrm{H} 30=0.9500 \\
& \mathrm{C} 31-\mathrm{H} 31=0.9500 \\
& \mathrm{C} 32-\mathrm{C} 33=1.479(2) \\
& \mathrm{C} 32-\mathrm{H} 32=0.9500 \\
& \mathrm{C} 33-\mathrm{C} 38=1.398(3) \\
& \mathrm{C} 33-\mathrm{C} 34=1.402(3) \\
& \mathrm{C} 34-\mathrm{C} 35=1.386(3) \\
& \mathrm{C} 34-\mathrm{H} 34=0.9500 \\
& \mathrm{C} 35-\mathrm{C} 36=1.384(3) \\
& \mathrm{C} 35-\mathrm{H} 35=0.9500 \\
& \mathrm{C} 36-\mathrm{C} 37=1.378(3) \\
& \mathrm{C} 36-\mathrm{H} 36=0.9500 \\
& \mathrm{C} 37-\mathrm{C} 38=1.387(3) \\
& \mathrm{C} 37-\mathrm{H} 37=0.9500 \\
& \mathrm{C} 38-\mathrm{H} 38=0.9500 \\
& \mathrm{C} 39-\mathrm{C} 46=1.347(2) \\
& \mathrm{C} 39-\mathrm{C} 40=1.492(2) \\
& \mathrm{C} 40-\mathrm{C} 45=1.391(3) \\
& \mathrm{C} 40-\mathrm{C} 41=1.393(3) \\
& \mathrm{C} 41-\mathrm{C} 42=1.394(3) \\
& \mathrm{C} 41-\mathrm{H} 41=0.9500 \\
& \mathrm{C} 42-\mathrm{C} 43=1.379(3) \\
& \mathrm{C} 42-\mathrm{H} 42=0.9500 \\
& \mathrm{C} 43-\mathrm{C} 44=1.379(3) \\
& \mathrm{C} 43-\mathrm{H} 43=0.9500 \\
& \mathrm{C} 44-\mathrm{C} 45=1.388(3) \\
& \mathrm{C} 44-\mathrm{H} 44=0.9500 \\
& \mathrm{C} 45-\mathrm{H} 45=0.9500 \\
& \mathrm{C} 46-\mathrm{C} 47=1.473(2) \\
& \mathrm{C} 46-\mathrm{H} 46=0.9500 \\
& \mathrm{C} 47-\mathrm{C} 52=1.397(3) \\
& \mathrm{C} 47-\mathrm{C} 48=1.402(3) \\
& \mathrm{C} 48-\mathrm{C} 49=1.382(3) \\
& \mathrm{C} 48-\mathrm{H} 48=0.9500 \\
& \mathrm{C} 49-\mathrm{C} 50=1.385(3) \\
& \mathrm{C} 49-\mathrm{H} 49=0.9500 \\
& \mathrm{C} 50-\mathrm{C} 51=1.377(3) \\
& \mathrm{C} 50-\mathrm{H} 50=0.9500
\end{aligned}
$$




\begin{tabular}{|c|c|c|}
\hline C51 & - C52 & $=1.387(3)$ \\
\hline C51 & $-\mathrm{H} 51$ & $=0.9500$ \\
\hline C52 & - H52 & $=0.9500$ \\
\hline P2 & - C65 & $=1.8445(17)$ \\
\hline P2 & - C53 & $=1.8662(18)$ \\
\hline & - C59 & $=1.8724(19)$ \\
\hline C53 & - C54 & $=1.536(2)$ \\
\hline C53 & - C58 & $=1.538(3)$ \\
\hline C53 & - H53 & $=1.0000$ \\
\hline C54 & - C55 & $=1.531(3)$ \\
\hline C54 & $-\mathrm{H} 54 \mathrm{~A}$ & $=0.9900$ \\
\hline C54 & $-\mathrm{H} 54 \mathrm{~B}$ & $=0.9900$ \\
\hline C55 & - C56 & $=1.522(3)$ \\
\hline C55 & $-H 55 A$ & $=0.9900$ \\
\hline C55 & $-H 55 B$ & $=0.9900$ \\
\hline C56 & - C57 & $=1.524(3)$ \\
\hline C56 & $-H 56 A$ & $=0.9900$ \\
\hline C56 & $-\mathrm{H} 56 \mathrm{~B}$ & $=0.9900$ \\
\hline C57 & - C58 & $=1.528(3)$ \\
\hline C57 & $-\mathrm{H} 57 \mathrm{~A}$ & $=0.9900$ \\
\hline C57 & - H57B & $=0.9900$ \\
\hline C58 & $-H 58 A$ & $=0.9900$ \\
\hline C58 & $-\mathrm{H} 58 \mathrm{~B}$ & $=0.9900$ \\
\hline C59 & $-\mathrm{C} 60$ & $=1.536(3)$ \\
\hline C59 & - C64 & $=1.538(2)$ \\
\hline C59 & - H59 & $=1.0000$ \\
\hline $\mathrm{C} 60$ & - C61 & $=1.527(3)$ \\
\hline $\mathrm{C} 60$ & - $\mathrm{H} 60 \mathrm{~A}$ & $=0.9900$ \\
\hline C60 & - H60B & $=0.9900$ \\
\hline C61 & $-\mathrm{c} 62$ & $=1.526(3)$ \\
\hline C61 & - H61A & $=0.9900$ \\
\hline C61 & $-\mathrm{H} 61 \mathrm{~B}$ & $=0.9900$ \\
\hline C62 & $-\mathrm{C} 63$ & $=1.518(3)$ \\
\hline C62 & - H62A & $=0.9900$ \\
\hline C62 & - H62B & $=0.9900$ \\
\hline C63 & - C64 & $=1.530(3)$ \\
\hline $\mathrm{C} 63$ & $-H 63 A$ & $=0.9900$ \\
\hline C63 & $-H 63 B$ & $=0.9900$ \\
\hline C64 & - H64A & $=0.9900$ \\
\hline C64 & $-H 64 B$ & $=0.9900$ \\
\hline C65 & - C70 & $=1.406(2)$ \\
\hline C65 & - C66 & $=1.409(2)$ \\
\hline C66 & $5-C 67$ & $=1.401(2)$ \\
\hline C66 & - C71 & $=1.508(2)$ \\
\hline C67 & 7- C68 & $=1.382(2)$ \\
\hline C67 & - H67 & $=0.9500$ \\
\hline C68 & - C69 & $=1.386(3)$ \\
\hline C68 & 3- H68 & $=0.9500$ \\
\hline C69 & - C70 & $=1.378(3)$ \\
\hline C69 & - H69 & $=0.9500$ \\
\hline C70 & $-\mathrm{H} 7 \mathrm{O}$ & $=0.9500$ \\
\hline C71 & - C72 & $=1.408(2)$ \\
\hline
\end{tabular}




$$
\begin{aligned}
& \mathrm{C} 71-\mathrm{C} 76=1.409(2) \\
& \mathrm{C} 72-\mathrm{C} 73=1.402(2) \\
& \mathrm{C} 72-\mathrm{C} 91=1.500(2) \\
& \mathrm{C} 73-\mathrm{C} 74=1.379(3) \\
& \mathrm{C} 73-\mathrm{H} 73=0.9500 \\
& \mathrm{C} 74-\mathrm{C} 75=1.386(3) \\
& \mathrm{C} 74-\mathrm{H} 74=0.9500 \\
& \mathrm{C} 75-\mathrm{C} 76=1.398(2) \\
& \mathrm{C} 75-\mathrm{H} 75=0.9500 \\
& \mathrm{C} 76-\mathrm{C} 77=1.496(2) \\
& \mathrm{C} 77-\mathrm{C} 84=1.347(2) \\
& \mathrm{C} 77-\mathrm{C} 78=1.489(2) \\
& \mathrm{C} 78-\mathrm{C} 79=1.391(2) \\
& \mathrm{C} 78-\mathrm{C} 83=1.396(2) \\
& \mathrm{C} 79-\mathrm{C} 80=1.384(3) \\
& \mathrm{C} 79-\mathrm{H} 79=0.9500 \\
& \mathrm{C} 80-\mathrm{C} 81=1.386(3) \\
& \mathrm{C} 80-\mathrm{H} 80=0.9500 \\
& \mathrm{C} 81-\mathrm{C} 82=1.380(3) \\
& \mathrm{C} 81-\mathrm{H} 81=0.9500 \\
& \mathrm{C} 82-\mathrm{C} 83=1.391(3) \\
& \mathrm{C} 82-\mathrm{H} 82=0.9500 \\
& \mathrm{C} 83-\mathrm{H} 83=0.9500 \\
& \mathrm{C} 84-\mathrm{C} 85=1.475(2) \\
& \mathrm{C} 84-\mathrm{H} 84=0.9500 \\
& \mathrm{C} 85-\mathrm{C} 86=1.395(3) \\
& \mathrm{C} 85-\mathrm{C} 90=1.396(3) \\
& \mathrm{C} 86-\mathrm{C} 87=1.385(3) \\
& \mathrm{C} 86-\mathrm{H} 86=0.9500 \\
& \mathrm{C} 87-\mathrm{C} 88=1.386(3) \\
& \mathrm{C} 87-\mathrm{H} 87=0.9500 \\
& \mathrm{C} 88-\mathrm{C} 89=1.379(3) \\
& \mathrm{C} 88-\mathrm{H} 88=0.9500 \\
& \mathrm{C} 89-\mathrm{C} 90=1.391(3) \\
& \mathrm{C} 89-\mathrm{H} 89=0.9500 \\
& \mathrm{C} 90-\mathrm{H} 90=0.9500 \\
& \mathrm{C} 91-\mathrm{C} 98=1.352(2) \\
& \mathrm{C} 91-\mathrm{C} 92=1.491(2) \\
& \mathrm{C} 92-\mathrm{C} 97=1.392(3) \\
& \mathrm{C} 92-\mathrm{C} 93=1.396(3) \\
& \mathrm{C} 93-\mathrm{C} 94=1.384(3) \\
& \mathrm{C} 93-\mathrm{H} 93=0.9500 \\
& \mathrm{C} 94-\mathrm{C} 95=1.382(3) \\
& \mathrm{C} 94-\mathrm{H} 94=0.9500 \\
& \mathrm{C} 95-\mathrm{C} 96=1.373(3) \\
& \mathrm{C} 95-\mathrm{H} 95=0.9500 \\
& \mathrm{C} 96-\mathrm{C} 97=1.391(3) \\
& \mathrm{C} 96-\mathrm{H} 96=0.9500 \\
& \mathrm{C} 97-\mathrm{H} 97=0.9500 \\
& \mathrm{C} 98-\mathrm{C} 99=1.467(2) \\
& \mathrm{C} 98-\mathrm{H} 98=0.9500 \\
& \mathrm{C} 99-\mathrm{C} 100=1.401(3)
\end{aligned}
$$




$$
\begin{array}{ll}
\mathrm{C} 99-\mathrm{C} 104 & =1.405(2) \\
\mathrm{C} 100-\mathrm{C} 101 & =1.386(3) \\
\mathrm{C} 100-\mathrm{H} 100 & =0.9500 \\
\mathrm{C} 101-\mathrm{C} 102 & =1.382(3) \\
\mathrm{C} 101-\mathrm{H} 101 & =0.9500 \\
\mathrm{C} 102-\mathrm{C} 103 & =1.385(3) \\
\mathrm{C} 102-\mathrm{H} 102 & =0.9500 \\
\mathrm{C} 103-\mathrm{C} 104 & =1.377(3) \\
\mathrm{C} 103-\mathrm{H} 103 & =0.9500 \\
\mathrm{C} 104-\mathrm{H} 104 & =0.9500 \\
\mathrm{Cl} 1-\mathrm{C} 105 & =1.755(3) \\
\mathrm{Cl} 2-\mathrm{C} 105 & =1.759(3) \\
\mathrm{C} 105-\mathrm{H} 10 \mathrm{C} & =0.9900 \\
\mathrm{C} 105-\mathrm{H} 10 \mathrm{D} & =0.9900
\end{array}
$$

Table S9. Angles [ $\left.{ }^{\circ}\right]$ for $4 a a$.

$\begin{array}{llll}\mathrm{C} 13 & -\mathrm{P} 1 & -\mathrm{C} 7 & =99.27(8) \\ \mathrm{C} 13 & -\mathrm{P} 1 & -\mathrm{C} 1 & =102.15(8) \\ \mathrm{C} 7 & -\mathrm{P} 1 & -\mathrm{C} 1 & =106.75(9) \\ \mathrm{C} 6 & -\mathrm{C} 1 & -\mathrm{C} 2 & =110.10(18) \\ \mathrm{C} 6 & -\mathrm{C} 1 & -\mathrm{P} 1 & =109.75(14) \\ \mathrm{C} 2 & -\mathrm{C} 1 & -\mathrm{P} 1 & =108.15(13) \\ \mathrm{C} 6 & -\mathrm{C} 1 & -\mathrm{H} 1 & =109.60 \\ \mathrm{C} 2 & -\mathrm{C} 1 & -\mathrm{H} 1 & =109.60 \\ \mathrm{P} 1 & -\mathrm{C} 1 & -\mathrm{H} 1 & =109.60 \\ \mathrm{C} 3 & -\mathrm{C} 2 & -\mathrm{C} 1 & =113.28(17) \\ \mathrm{C} 3 & -\mathrm{C} 2 & -\mathrm{H} 2 \mathrm{~A} & =108.90 \\ \mathrm{C} 1 & -\mathrm{C} 2 & -\mathrm{H} 2 \mathrm{~A} & =108.90 \\ \mathrm{C} 3 & -\mathrm{C} 2 & -\mathrm{H} 2 \mathrm{~B} & =108.90 \\ \mathrm{C} 1 & -\mathrm{C} 2 & -\mathrm{H} 2 \mathrm{~B} & =108.90 \\ \mathrm{H} 2 \mathrm{~A} & -\mathrm{C} 2 & -\mathrm{H} 2 \mathrm{~B} & =107.70 \\ \mathrm{C} 4 & -\mathrm{C} 3 & -\mathrm{C} 2 & =111.55(18) \\ \mathrm{C} 4 & -\mathrm{C} 3 & -\mathrm{H} 3 \mathrm{~A} & =109.30 \\ \mathrm{C} 2 & -\mathrm{C} 3 & -\mathrm{H} 3 \mathrm{~A} & =109.30 \\ \mathrm{C} 4 & -\mathrm{C} 3 & -\mathrm{H} 3 \mathrm{~B} & =109.30 \\ \mathrm{C} 2 & -\mathrm{C} 3 & -\mathrm{H} 3 \mathrm{~B} & =109.30 \\ \mathrm{H} 3 \mathrm{~A} & -\mathrm{C} 3 & -\mathrm{H} 3 \mathrm{~B} & =108.00 \\ \mathrm{C} 5 & -\mathrm{C} 4 & -\mathrm{C} 3 & =110.00(2) \\ \mathrm{C} 5 & -\mathrm{C} 4 & -\mathrm{H} 4 \mathrm{~A} & =109.70 \\ \mathrm{C} 3 & -\mathrm{C} 4 & -\mathrm{H} 4 \mathrm{~A} & =109.70 \\ \mathrm{C} 5 & -\mathrm{C} 4 & -\mathrm{H} 4 \mathrm{~B} & =109.70 \\ \mathrm{C} 3 & -\mathrm{C} 4 & -\mathrm{H} 4 \mathrm{~B} & =109.70 \\ \mathrm{H} 4 \mathrm{~A} & -\mathrm{C} 4 & -\mathrm{H} 4 \mathrm{~B} & =108.20 \\ \mathrm{C} 4 & -\mathrm{C} 5 & -\mathrm{C} 6 & =111.30(2) \\ \mathrm{C} 4 & -\mathrm{C} 5 & -\mathrm{H} 5 \mathrm{~A} & =109.40 \\ \mathrm{C} 6 & -\mathrm{C} 5 & -\mathrm{H} 5 \mathrm{~A} & =109.40 \\ \mathrm{C} 4 & -\mathrm{C} 5 & -\mathrm{H} 5 \mathrm{~B} & =109.40 \\ \mathrm{C} 6 & -\mathrm{C} 5 & -\mathrm{H} 5 \mathrm{~B} & =109.40 \\ \mathrm{H} 5 \mathrm{~A} & -\mathrm{C} 5 & -\mathrm{H} 5 \mathrm{~B} & =108.00 \\ \mathrm{C} 5 & -\mathrm{C} 6 & -\mathrm{C} 1 & =112.46(19) \\ \mathrm{C} 5 & -\mathrm{C} 6 & -\mathrm{H} 6 \mathrm{~A} & =109.10 \\ & & & \end{array}$




$$
\begin{aligned}
& \mathrm{C} 1-\mathrm{C} 6-\mathrm{H} 6 \mathrm{~A}=109.10 \\
& \mathrm{C} 5-\mathrm{C} 6-\mathrm{H} 6 \mathrm{~B}=109.10 \\
& \mathrm{C} 1-\mathrm{C} 6-\mathrm{H} 6 \mathrm{~B}=109.10 \\
& \mathrm{H} 6 \mathrm{~A}-\mathrm{C} 6-\mathrm{H} 6 \mathrm{~B}=107.80 \\
& \mathrm{C} 8-\mathrm{C} 7-\mathrm{C} 12=110.02(17) \\
& \mathrm{C} 8-\mathrm{C} 7 \quad-\mathrm{P} 1=118.31(14) \\
& \mathrm{C} 12-\mathrm{C} 7-\mathrm{P} 1=111.81(14) \\
& \mathrm{C} 8-\mathrm{C} 7-\mathrm{H} 7=105.20 \\
& \mathrm{C} 12-\mathrm{C} 7-\mathrm{H} 7=105.20 \\
& \mathrm{P} 1-\mathrm{C} 7 \quad-\mathrm{H} 7=105.20 \\
& \mathrm{C} 7-\mathrm{C} 8-\mathrm{C} 9=110.96(18) \\
& \mathrm{C} 7-\mathrm{C} 8-\mathrm{H} 8 \mathrm{~A}=109.40 \\
& \mathrm{C} 9-\mathrm{C} 8-\mathrm{H} 8 \mathrm{~A}=109.40 \\
& \mathrm{C} 7-\mathrm{C} 8-\mathrm{H} 8 \mathrm{~B}=109.40 \\
& \mathrm{C} 9-\mathrm{C} 8-\mathrm{H} 8 \mathrm{~B}=109.40 \\
& \mathrm{H} 8 \mathrm{~A}-\mathrm{C} 8-\mathrm{H} 8 \mathrm{~B}=108.00 \\
& \mathrm{C} 10-\mathrm{C} 9-\mathrm{C} 8=111.00(2) \\
& \mathrm{C} 10-\mathrm{C} 9-\mathrm{H} 9 \mathrm{~A}=109.40 \\
& \mathrm{C} 8-\mathrm{C} 9-\mathrm{H} 9 \mathrm{~A}=109.40 \\
& \mathrm{C} 10-\mathrm{C} 9-\mathrm{H} 9 \mathrm{~B}=109.40 \\
& \mathrm{C} 8-\mathrm{C} 9-\mathrm{H9B}=109.40 \\
& \mathrm{H9A}-\mathrm{C9}-\mathrm{H9B}=108.00 \\
& \mathrm{C} 11-\mathrm{C} 10-\mathrm{C} 9=111.10(19) \\
& \mathrm{C} 11-\mathrm{C} 10-\mathrm{H} 10 \mathrm{~A}=109.40 \\
& \mathrm{C} 9-\mathrm{C} 10-\mathrm{H} 10 \mathrm{~A}=109.40 \\
& \mathrm{C} 11-\mathrm{C} 10-\mathrm{H} 10 \mathrm{~B}=109.40 \\
& \mathrm{C} 9-\mathrm{C} 10-\mathrm{H} 10 \mathrm{~B}=109.40 \\
& \mathrm{H} 10 \mathrm{~A}-\mathrm{C} 10-\mathrm{H} 10 \mathrm{~B}=108.00 \\
& \mathrm{C} 10-\mathrm{C} 11-\mathrm{C} 12=111.61(18) \\
& \mathrm{C} 10-\mathrm{C} 11-\mathrm{H} 11 \mathrm{~A}=109.30 \\
& \mathrm{C} 12-\mathrm{C} 11-\mathrm{H} 11 \mathrm{~A}=109.30 \\
& \mathrm{C} 10-\mathrm{C} 11-\mathrm{H} 11 \mathrm{~B}=109.30 \\
& \mathrm{C} 12-\mathrm{C} 11-\mathrm{H} 11 \mathrm{~B}=109.30 \\
& \mathrm{H} 11 \mathrm{~A}-\mathrm{C} 11-\mathrm{H} 11 \mathrm{~B}=108.00 \\
& \mathrm{C} 11-\mathrm{C} 12-\mathrm{C} 7 \quad=111.76(17) \\
& \mathrm{C} 11-\mathrm{C} 12-\mathrm{H} 12 \mathrm{~A}=109.30 \\
& \mathrm{C} 7-\mathrm{C} 12-\mathrm{H} 12 \mathrm{~A}=109.30 \\
& \mathrm{C} 11-\mathrm{C} 12-\mathrm{H} 12 \mathrm{~B}=109.30 \\
& \mathrm{C} 7-\mathrm{C} 12-\mathrm{H} 12 \mathrm{~B}=109.30 \\
& \mathrm{H} 12 \mathrm{~A}-\mathrm{C} 12-\mathrm{H} 12 \mathrm{~B}=107.90 \\
& \mathrm{C} 14-\mathrm{C} 13-\mathrm{C} 18=118.07(16) \\
& \mathrm{C} 14-\mathrm{C} 13-\mathrm{P} 1=122.50(14) \\
& \mathrm{C} 18-\mathrm{C} 13-\mathrm{P} 1=119.44(13) \\
& \mathrm{C} 15-\mathrm{C} 14-\mathrm{C} 13=121.71(18) \\
& \mathrm{C} 15-\mathrm{C} 14-\mathrm{H} 14=119.10 \\
& \mathrm{C} 13-\mathrm{C} 14-\mathrm{H} 14=119.10 \\
& \mathrm{C} 16-\mathrm{C} 15-\mathrm{C} 14=119.70(17) \\
& \mathrm{C} 16-\mathrm{C} 15-\mathrm{H} 15=120.20 \\
& \mathrm{C} 14-\mathrm{C} 15-\mathrm{H} 15=120.20 \\
& \mathrm{C} 17-\mathrm{C} 16-\mathrm{C} 15=119.66(17) \\
& \mathrm{C} 17-\mathrm{C} 16-\mathrm{H} 16=120.20 \\
& \mathrm{C} 15-\mathrm{C} 16-\mathrm{H} 16=120.20
\end{aligned}
$$




\begin{tabular}{|c|c|c|c|}
\hline C16 & - C17 & - C18 & $=121.24(17)$ \\
\hline & - C17 & - H17 & $=119.40$ \\
\hline & - C17 & - H17 & $=119.40$ \\
\hline 17 & - C18 & - C13 & $=119.52(15)$ \\
\hline 17 & - C18 & - C19 & $=119.47(15)$ \\
\hline 13 & - C18 & - C19 & $=120.99(15)$ \\
\hline 24 & - C19 & $-\mathrm{C} 20$ & $=119.34(15)$ \\
\hline 24 & C19 & - C18 & $=119$. \\
\hline 20 & - C19 & - C18 & $=120$. \\
\hline 21 & - C2O & - C19 & $=11$ \\
\hline 21 & $-\mathrm{C} 20$ & $-\mathrm{C} 25$ & $=11$ \\
\hline 19 & $-\mathrm{C} 20$ & $-\mathrm{C} 25$ & 15) \\
\hline 22 & $-\mathrm{C} 21$ & $-\mathrm{C} 20$ & $=1$ \\
\hline 22 & $-\mathrm{C} 21$ & $-\mathrm{H} 21$ & $=1$ \\
\hline 20 & $-\mathrm{C} 21$ & - H21 & $=1$ \\
\hline 21 & $-\mathrm{C} 22$ & $-\mathrm{C} 23$ & $=11$ \\
\hline 1 & $-\mathrm{C} 22$ & $-\mathrm{H} 22$ & \\
\hline 6 & $-\mathrm{C} 22$ & $\mathrm{H} 22$ & \\
\hline 2 & $-\mathrm{C} 23$ & - C24 & $=1$ \\
\hline 2 & $-\mathrm{C} 23$ & $-\mathrm{H} 23$ & $=1$ \\
\hline 24 & $-\mathrm{C} 23$ & $-\mathrm{H} 23$ & $=1$ \\
\hline & $-\mathrm{C} 24$ & - C19 & \\
\hline & $-\mathrm{C} 24$ & - C39 & 15) \\
\hline 1. & $-\mathrm{C} 24$ & C39 & $=1$ \\
\hline C32 & $-\mathrm{C} 25$ & $-\mathrm{C} 26$ & $=1$ \\
\hline 32 & $-\mathrm{C} 25$ & $-\mathrm{C} 20$ & $=1$ \\
\hline & $-\mathrm{C} 25$ & - C20 & \\
\hline ד & $-\mathrm{C} 26$ & - C31 & \\
\hline 27 & $-\mathrm{C} 26$ & $-\mathrm{C} 25$ & \\
\hline 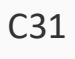 & $-\mathrm{C} 26$ & $-\mathrm{C} 25$ & $=1$ \\
\hline C26 & $-\mathrm{C} 27$ & $-\mathrm{C} 28$ & $=1$ \\
\hline 26 & $-\mathrm{C} 27$ & $-\mathrm{H} 27$ & \\
\hline & $-\mathrm{C} 27$ & - $\mathrm{H} 27$ & \\
\hline C29 & $-\mathrm{C} 28$ & $-\mathrm{C} 27$ & $=1$ \\
\hline $\mathrm{C} 29$ & $-\mathrm{C} 28$ & $-\mathrm{H} 28$ & $=$ \\
\hline 27.27 & $-\mathrm{C} 28$ & $\mathrm{H} 28$ & $=$ \\
\hline 28 & $-\mathrm{C} 29$ & $-\mathrm{C} 30$ & $7(18)$ \\
\hline & $-C 29$ & $-\mathrm{H} 29$ & $=1$ \\
\hline & $-\mathrm{C} 29$ & $-\mathrm{H} 29$ & \\
\hline & $-\mathrm{C} 30$ & $-\mathrm{C} 31$ & \\
\hline $\mathrm{C} 2 \mathrm{~S}$ & - C30 & $-\mathrm{H} 30$ & $=1$ \\
\hline C31 & $-\mathrm{C} 30$ & $-\mathrm{H} 30$ & $=1$ \\
\hline C30 & - C31 & $-\mathrm{C} 26$ & $=12$ \\
\hline & - C31 & $-\mathrm{H} 31$ & $=1$ \\
\hline & $-\mathrm{C} 31$ & $-\mathrm{H} 31$ & $=1$ \\
\hline $\mathrm{C} 25$ & $-\mathrm{C} 32$ & $-\mathrm{C} 33$ & $=12$ \\
\hline $\mathrm{C} 25$ & $-\mathrm{C} 32$ & $-\mathrm{H} 32$ & $=11$ \\
\hline C33 & $-\mathrm{C} 32$ & $-\mathrm{H} 32$ & $=115$ \\
\hline C38 & $-\mathrm{C} 33$ & - C34 & $=117.78(17)$ \\
\hline C38 & $-\mathrm{C} 33$ & $-\mathrm{C} 32$ & \\
\hline & & - C32 & - \\
\hline & - C34 & - C33 & $=120.68(19)$ \\
\hline
\end{tabular}




\begin{tabular}{|c|c|c|c|}
\hline C35 & - C34 & - H34 & $=119.70$ \\
\hline C33 & - C34 & $-\mathrm{H} 34$ & $=119.70$ \\
\hline & - C35 & - C34 & $=120.50(2)$ \\
\hline 6 & $-\mathrm{C} 35$ & $-\mathrm{H} 35$ & $=119.70$ \\
\hline 34 & $-\mathrm{C} 35$ & $-\mathrm{H} 35$ & $=119.70$ \\
\hline 37 & $-\mathrm{C} 36$ & - C35 & $=119.65(19)$ \\
\hline 37 & $-\mathrm{C} 36$ & $-\mathrm{H} 36$ & $=120.20$ \\
\hline 35 & $-\mathrm{C} 36$ & $-\mathrm{H} 36$ & $=120.20$ \\
\hline 36 & $-\mathrm{C} 37$ & $-\mathrm{C} 38$ & $=120.20(2)$ \\
\hline 36 & - C37 & - H37 & $=119.90$ \\
\hline 38 & - C37 & $-\mathrm{H} 37$ & $=119.90$ \\
\hline 37 & $-\mathrm{C} 38$ & - C33 & $=121.14(19)$ \\
\hline 37 & - C38 & $-\mathrm{H} 38$ & $=119.40$ \\
\hline 33 & $-\mathrm{C} 38$ & $-\mathrm{H} 38$ & 19.40 \\
\hline 46 & - C39 & - C40 & $1.61(16)$ \\
\hline E46 & - C39 & $-C 24$ & $=1$ \\
\hline 40 & - C39 & - C24 & $=120.04(15)$ \\
\hline 45 & $-\mathrm{C} 40$ & - C41 & $=118.48(17)$ \\
\hline C45 & - C40 & - С39 & $=120.58(16)$ \\
\hline C41 & - C40 & - С39 & $0.66(16)$ \\
\hline $\mathrm{C} 40$ & $-\mathrm{C} 41$ & $-\mathrm{C} 42$ & $=1$ \\
\hline $\mathrm{C} 40$ & - C41 & - H41 & $=119.70$ \\
\hline C42 & - C41 & - H41 & 9.70 \\
\hline C43 & $-\mathrm{C} 42$ & $-\mathrm{C} 41$ & $=120.30(2)$ \\
\hline C43 & $-\mathrm{C} 42$ & $-\mathrm{H} 42$ & $=119.90$ \\
\hline C41 & $-\mathrm{C} 42$ & $-\mathrm{H} 42$ & 9.90 \\
\hline C42 & $-\mathrm{C} 43$ & - C44 & $9.43(19)$ \\
\hline C42 & - C43 & $-\mathrm{H} 43$ & $=120.30$ \\
\hline C44 & $-\mathrm{C} 43$ & $-\mathrm{H} 43$ & $=1$ \\
\hline $\mathrm{C} 43$ & - C44 & $-\mathrm{C} 45$ & $=120.70(2)$ \\
\hline $\mathrm{C} 43$ & - C44 & - H44 & $=119.70$ \\
\hline C45 & $-\mathrm{C} 44$ & $-\mathrm{H} 44$ & $=1$ \\
\hline C44 & $-\mathrm{C} 45$ & $-\mathrm{C} 40$ & $=120.52(19)$ \\
\hline C44 & - C45 & - $\mathrm{H} 45$ & $=119.70$ \\
\hline $\mathrm{C} 40$ & $-\mathrm{C} 45$ & $-\mathrm{H} 45$ & $=119.70$ \\
\hline C39 & $-\mathrm{C} 46$ & $-\mathrm{C} 47$ & $9.31(17)$ \\
\hline C39 & $-\mathrm{C} 46$ & $-\mathrm{H} 46$ & 5.30 \\
\hline C47 & $-\mathrm{C} 46$ & $-\mathrm{H} 46$ & $=115.30$ \\
\hline C52 & - C47 & - C48 & $=117.53(17)$ \\
\hline C52 & $-\mathrm{C} 47$ & $-\mathrm{C} 46$ & $=118.64(18)$ \\
\hline $\mathrm{C} 48$ & $-\mathrm{C} 47$ & $-\mathrm{C} 46$ & $23.80(17)$ \\
\hline C49 & $-\mathrm{C} 48$ & $-\mathrm{C} 47$ & $20.98(18)$ \\
\hline C49 & $-\mathrm{C} 48$ & $-\mathrm{H} 48$ & 19.50 \\
\hline C47 & - C48 & $-\mathrm{H} 48$ & $=119.50$ \\
\hline C48 & - C49 & - C50 & $=120.10(2)$ \\
\hline C48 & $-C 49$ & $-\mathrm{H} 49$ & $=119.90$ \\
\hline C50 & - C49 & $-\mathrm{H} 49$ & $=119.90$ \\
\hline C51 & $-\mathrm{C} 50$ & $-C 49$ & $=120.07(19)$ \\
\hline C51 & $-\mathrm{C} 50$ & $-\mathrm{H} 50$ & $=120.00$ \\
\hline C49 & $-\mathrm{C} 50$ & $-\mathrm{H} 50$ & $=120.00$ \\
\hline $\mathrm{C} 50$ & $-\mathrm{C} 51$ & $-\mathrm{C} 52$ & $=119.80(19)$ \\
\hline C50 & - C51 & - H51 & $=120.10$ \\
\hline
\end{tabular}




\begin{tabular}{|c|c|c|c|}
\hline 52 & - C51 & - H51 & $=120.10$ \\
\hline 51 & $-\mathrm{C} 52$ & $-\mathrm{C} 47$ & $=121.40(2)$ \\
\hline 51 & - C52 & $-\mathrm{H} 52$ & $=119.30$ \\
\hline 47 & - C52 & $-\mathrm{H} 52$ & $=119.30$ \\
\hline 5 & $-P 2$ & $-\mathrm{C} 53$ & $=101.96(8)$ \\
\hline 65 & $-P 2$ & - C59 & $=99.97(8)$ \\
\hline 53 & $-\mathrm{P} 2$ & $-\mathrm{C} 59$ & $=105.34(8)$ \\
\hline 54 & - C53 & - C58 & $=109.91(15)$ \\
\hline 54 & $-\mathrm{C} 53$ & $-P 2$ & $=110.92(12)$ \\
\hline 58 & $-\mathrm{C} 53$ & $-\mathrm{P} 2$ & $=108.49(12)$ \\
\hline 54 & - C53 & $-\mathrm{H} 53$ & $=109.20$ \\
\hline 58 & $-\mathrm{C} 53$ & $-\mathrm{H} 53$ & $=109.20$ \\
\hline 2 & $-\mathrm{C53}$ & $-\mathrm{H} 53$ & $=109.20$ \\
\hline 55 & - C54 & - C53 & $=111.32(16)$ \\
\hline C55 & $-\mathrm{C} 54$ & $-\mathrm{H} 54 \mathrm{~A}$ & $=109.40$ \\
\hline 53 & $-\mathrm{C} 54$ & - H54A & $=109.40$ \\
\hline 55 & - C54 & $-\mathrm{H} 54 \mathrm{~B}$ & $=109.40$ \\
\hline 53 & - C54 & - H54B & $=109.40$ \\
\hline $54 \mathrm{~A}$ & - C54 & - H54B & $B=108.00$ \\
\hline 56 & - C55 & - C54 & $=111.65(17)$ \\
\hline 56 & - C55 & - H55A & $=109.30$ \\
\hline 54 & - C55 & $-\mathrm{H} 55 \mathrm{~A}$ & $=109.30$ \\
\hline 56 & $-\mathrm{C} 55$ & $-\mathrm{H} 55 \mathrm{~B}$ & $=109.30$ \\
\hline 54 & - C55 & $-\mathrm{H} 55 \mathrm{~B}$ & $=109.30$ \\
\hline $55 \mathrm{~A}$ & - C55 & - H55B & $B=108.00$ \\
\hline C55 & - C56 & $-\mathrm{C} 57$ & $=110.90(16)$ \\
\hline 55 & - C56 & $-H 56 A$ & $=109.50$ \\
\hline C57 & - C56 & $-H 56 A$ & $=109.50$ \\
\hline 55 & $-\mathrm{C} 56$ & $-\mathrm{H} 56 \mathrm{~B}$ & $=109.50$ \\
\hline C57 & $-\mathrm{C} 56$ & $-H 56 B$ & $=109.50$ \\
\hline H56A & - C56 & - H56B & $3=108.00$ \\
\hline 56 & - C57 & - C58 & $=111.21(16$ \\
\hline 56 & - C57 & $-\mathrm{H} 57 \mathrm{~A}$ & $=109.40$ \\
\hline C58 & $-\mathrm{C} 57$ & - H57A & $=109.40$ \\
\hline C56 & $-\mathrm{C} 57$ & - H57B & $=109.40$ \\
\hline C58 & - C57 & - H57B & $=109.40$ \\
\hline $57 \mathrm{~A}$ & $-\mathrm{C} 57$ & - H57B & $3=108.00$ \\
\hline C57 & $-\mathrm{C} 58$ & $-\mathrm{C} 53$ & $=113.02(15)$ \\
\hline C57 & - C58 & - H58A & $=109.00$ \\
\hline C53 & - C58 & $-\mathrm{H} 58 \mathrm{~A}$ & $=109.00$ \\
\hline C57 & $-\mathrm{C} 58$ & $-\mathrm{H} 58 \mathrm{~B}$ & $=109.00$ \\
\hline C53 & $-\mathrm{C} 58$ & - H58B & $=109.00$ \\
\hline H58A & - C58 & - H58B & $3=107.80$ \\
\hline C60 & - C59 & $-\mathrm{C} 64$ & $=110.61(15)$ \\
\hline C60 & - C59 & $-P 2$ & $=118.05(13)$ \\
\hline C64 & - C59 & $-\mathrm{P} 2$ & $=110.95(13)$ \\
\hline C60 & - C59 & $-\mathrm{H} 59$ & $=105.40$ \\
\hline C64 & - C59 & - H59 & $=105.40$ \\
\hline P2 & $-\mathrm{C59}$ & $-\mathrm{H} 59$ & $=105.40$ \\
\hline C61 & - C60 & - C59 & $=111.88(16)$ \\
\hline C61 & - C60 & - H60A & $=109.20$ \\
\hline C59 & $-\mathrm{C} 60$ & $-\mathrm{H} 60 \mathrm{~A}$ & $=109.20$ \\
\hline
\end{tabular}




\begin{tabular}{|c|c|c|c|}
\hline 61 & - C60 & - H60B & $=109.20$ \\
\hline 59 & $-\mathrm{C} 60$ & - H60B & $=109.20$ \\
\hline SOA & - C60 & $-\mathrm{H} 60 \mathrm{~B}$ & $=107.90$ \\
\hline 62 & - C61 & - C60 & $=111.47(18$ \\
\hline 62 & - C61 & - H61A & $=109.30$ \\
\hline 60 & - C61 & - H61A & $=109.30$ \\
\hline 62 & - C61 & $-H 61 B$ & $=109.30$ \\
\hline 0 & $-\mathrm{C} 61$ & $-\mathrm{H} 61 \mathrm{~B}$ & $=109.30$ \\
\hline $61 \mathrm{~A}$ & - C61 & - H61B & $=108.00$ \\
\hline 63 & - C62 & - C61 & $=110.67(16)$ \\
\hline 63 & - C62 & - H62A & $=109.50$ \\
\hline 61 & $-\mathrm{C} 62$ & $-\mathrm{H} 62 \mathrm{~A}$ & $=109.50$ \\
\hline 53 & $-\mathrm{C} 62$ & - H62B & $=109.50$ \\
\hline 51 & $-\mathrm{C} 62$ & $-\mathrm{H} 62 \mathrm{~B}$ & $=109.50$ \\
\hline $62 \mathrm{~A}$ & - C62 & - H62B & $=108.10$ \\
\hline 62 & $-\mathrm{C} 63$ & - C64 & $=111.67(16)$ \\
\hline 52 & - C63 & - H63A & $=109.30$ \\
\hline 54 & $-\mathrm{C} 63$ & $-\mathrm{H} 63 \mathrm{~A}$ & $=109.30$ \\
\hline 52 & $-\mathrm{C} 63$ & $-\mathrm{H} 63 B$ & $=109.30$ \\
\hline 54 & $-\mathrm{C} 63$ & $-\mathrm{H} 63 \mathrm{~B}$ & $=10 s$ \\
\hline $63 \mathrm{~A}$ & - C63 & - H63B & $=107.90$ \\
\hline 53 & - C64 & - C59 & $=112.08(16)$ \\
\hline & $-\mathrm{C} 64$ & - H64A & $=109.20$ \\
\hline 59 & - C64 & - H64A & $=109.20$ \\
\hline 53 & - C64 & - H64B & $=109.20$ \\
\hline 9 & - C64 & $-H 64 B$ & $=109.20$ \\
\hline $54 \mathrm{~A}$ & - C64 & - H64B & $3=107.90$ \\
\hline 70 & $-\mathrm{C} 65$ & - C66 & $=117.7$ \\
\hline 70 & $-\mathrm{C} 65$ & $-P 2$ & $=121.8$ \\
\hline 56 & $-\mathrm{C} 65$ & $-P 2$ & $=120.3$ \\
\hline 67 & - C66 & - C65 & $=119.2$ \\
\hline 67 & - C66 & - C71 & $=115 . \mathrm{c}$ \\
\hline & - C66 & - C71 & $=124.6$ \\
\hline 68 & - C67 & - C66 & $=121.63(16)$ \\
\hline 58 & - C67 & - H67 & $=119$. \\
\hline 66 & $-\mathrm{C} 67$ & - H67 & $=119.2$ \\
\hline 67 & $-\mathrm{C} 68$ & $-C 69$ & $=119.20(17)$ \\
\hline 67 & - C68 & $-\mathrm{H} 68$ & $=120$. \\
\hline C69 & - C68 & - H68 & $=120.4$ \\
\hline & $-C 69$ & $-\mathrm{C} 68$ & $=120$. \\
\hline C & - C69 & - H69 & $=120$. \\
\hline C68 & - C69 & - H69 & $=120$. \\
\hline C69 & - C70 & - C65 & $=122.01(17)$ \\
\hline 69 & - C70 & - H70 & $=119$. \\
\hline & - C70 & - H70 & $=119$. \\
\hline $\mathrm{C} 72$ & - C71 & - C76 & $=118.6$ \\
\hline $\mathrm{C} 72$ & - C71 & - C66 & $=121.94$ \\
\hline C76 & $-\mathrm{C} 71$ & - C66 & $=119.36(14)$ \\
\hline C73 & - C72 & - C71 & $=119.60(16)$ \\
\hline C73 & - C72 & - C91 & $=116.78(15)$ \\
\hline & $-\mathrm{C} 72$ & - C91 & $=123.52(15)$ \\
\hline C7L & - C73 & - C72 & $=121.50(16)$ \\
\hline
\end{tabular}




\begin{tabular}{|c|c|c|c|}
\hline C74 & - C73 & - H73 & $=119.30$ \\
\hline C72 & - C73 & $-\mathrm{H} 73$ & $=119.30$ \\
\hline 73 & - C74 & - C75 & $=119.06(16$ \\
\hline 3 & - C74 & - H74 & $=120.50$ \\
\hline 75 & - C74 & - H74 & $=120.50$ \\
\hline 74 & - C75 & - C76 & $=121.05(17)$ \\
\hline 74 & - C75 & $-\mathrm{H} 75$ & $=119.50$ \\
\hline 76 & - C75 & - H75 & $=119.50$ \\
\hline 75 & - C76 & - C71 & $=120.02(16)$ \\
\hline 75 & - C76 & - C77 & $=117.33(15)$ \\
\hline 71 & - C76 & - C77 & $=122.59(15)$ \\
\hline 84 & $-\mathrm{C} 77$ & - C78 & $=125.36(16)$ \\
\hline C84 & $-\mathrm{C} 77$ & - C76 & $=117.35$ \\
\hline C78 & $-\mathrm{C} 77$ & - C76 & $=1$ \\
\hline C79 & $-\mathrm{C} 78$ & $-\mathrm{C} 83$ & $=1$ \\
\hline C79 & $-\mathrm{C} 78$ & - C77 & $=1$ \\
\hline 83 & - C78 & - C77 & $=120.17(16)$ \\
\hline $\mathrm{C} 80$ & - C79 & $-\mathrm{C} 78$ & $=120.96(17)$ \\
\hline $\mathrm{C} 80$ & - C79 & - H79 & $=119.50$ \\
\hline C78 & - C79 & - H79 & $=1$ \\
\hline C79 & $-\mathrm{C} 80$ & $-\mathrm{C} 81$ & $=1$ \\
\hline C79 & $-\mathrm{C} 80$ & $-\mathrm{H} 80$ & $=1$ \\
\hline C81 & $-\mathrm{C} 80$ & $-\mathrm{H} 80$ & 20.00 \\
\hline C82 & $-C 81$ & $-\mathrm{C} 80$ & $=119.79(18)$ \\
\hline C82 & $-C 81$ & $-\mathrm{H} 81$ & 20.10 \\
\hline $\mathrm{C} 80$ & $-\mathrm{C} 81$ & $-\mathrm{H} 81$ & 0.10 \\
\hline C81 & $-C 82$ & $-\mathrm{C} 83$ & $0.44(18)$ \\
\hline 81 & $-\mathrm{C} 82$ & - H82 & $=119.80$ \\
\hline C83 & $-\mathrm{C} 82$ & $-\mathrm{H} 82$ & $=1$ \\
\hline C82 & $-C 83$ & $-\mathrm{C} 78$ & $=120.18(18)$ \\
\hline C82 & $-\mathrm{C} 83$ & $-\mathrm{H} 83$ & 19.90 \\
\hline C78 & $-\mathrm{C} 83$ & $-\mathrm{H} 83$ & $=1$ \\
\hline C77 & $-\mathrm{C} 84$ & $-\mathrm{C} 85$ & $0.98(17)$ \\
\hline 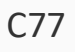 & $-\mathrm{C} 84$ & - H84 & \\
\hline C85 & $-\mathrm{C} 84$ & - H84 & $=1$ \\
\hline C86 & $-\mathrm{C} 85$ & - C90 & $=1$ \\
\hline C86 & $-\mathrm{C} 85$ & $-\mathrm{C} 84$ & $=1$ \\
\hline $\mathrm{C} 90$ & $-\mathrm{C} 85$ & $-\mathrm{C} 84$ & $=124.15(17)$ \\
\hline C87 & $-\mathrm{C} 86$ & $-\mathrm{C} 85$ & $=121.26(19)$ \\
\hline C87 & $-\mathrm{C} 86$ & $-\mathrm{H} 86$ & $=1$ \\
\hline C85 & $-C 86$ & $-\mathrm{H} 86$ & $=1$ \\
\hline C86 & $-\mathrm{C} 87$ & $-\mathrm{C} 88$ & $0.10(2)$ \\
\hline C86 & $-\mathrm{C} 87$ & $-\mathrm{H} 87$ & $=1$ \\
\hline C88 & $-\mathrm{C} 87$ & $-\mathrm{H} 87$ & $=120.00$ \\
\hline C89 & $-\mathrm{C} 88$ & $-\mathrm{C} 87$ & $=119.64(18)$ \\
\hline $\mathrm{C} 89$ & $-\mathrm{C} 88$ & $-\mathrm{H} 88$ & $=120.20$ \\
\hline C87 & $-C 88$ & $-\mathrm{H} 88$ & $=120.20$ \\
\hline C88 & $-C 89$ & - C90 & $=120.28(19$ \\
\hline C88 & $-C 89$ & $-\mathrm{H} 89$ & $=119.90$ \\
\hline C90 & $-C 89$ & $-\mathrm{H} 89$ & $=119.90$ \\
\hline $\mathrm{C} 8 \mathrm{~S}$ & - C90 & $-\mathrm{C} 85$ & $=120.91(1$ \\
\hline C89 & - C90 & - H9O & $=119.50$ \\
\hline
\end{tabular}




\begin{tabular}{|c|c|c|c|}
\hline C85 & - C90 & - H9O & $=119.50$ \\
\hline C98 & - C91 & - C92 & $=128.81(16)$ \\
\hline C98 & - C91 & $-\mathrm{C} 72$ & $=114.08(15)$ \\
\hline C92 & - C91 & - C72 & $=116.86(14)$ \\
\hline C97 & - C92 & - C93 & $=118.01(17)$ \\
\hline C97 & - C92 & - C91 & $=122.95(16)$ \\
\hline C93 & - C92 & - C91 & $=118.98(16)$ \\
\hline C94 & - C93 & - C92 & $=121.10(19)$ \\
\hline C94 & - C93 & $-\mathrm{H} 93$ & $=119.40$ \\
\hline C92 & - C93 & $-\mathrm{H} 93$ & $=119.40$ \\
\hline C95 & - C94 & - C93 & $=120.00(2)$ \\
\hline C95 & - C94 & - H94 & $=120.00$ \\
\hline C93 & - C94 & - H94 & $=120.00$ \\
\hline C96 & - C95 & - C94 & $=119.80(19)$ \\
\hline C96 & - C95 & - H95 & $=120.10$ \\
\hline C94 & - C95 & - H95 & $=120.10$ \\
\hline C95 & - C96 & - C97 & $=120.50(2)$ \\
\hline C95 & - C96 & - H96 & $=119.80$ \\
\hline C97 & - C96 & - H96 & $=119.80$ \\
\hline C96 & - C97 & - C92 & $=120.60(19)$ \\
\hline C96 & - C97 & - H97 & $=119.70$ \\
\hline C92 & - C97 & - H97 & $=119.70$ \\
\hline C91 & - C98 & - C99 & $=134.66(17)$ \\
\hline C91 & - C98 & - H98 & $=112.70$ \\
\hline C99 & - C98 & - H98 & $=112.70$ \\
\hline C100 & - C99 & - C104 & $=117.65(16)$ \\
\hline C100 & - C99 & - C98 & $=127.01(16)$ \\
\hline C104 & - C99 & - C98 & $=115.23(16)$ \\
\hline C101 & - C100 & - C99 & $=120.26(17)$ \\
\hline C101 & - C100 & - H100 & $=119.90$ \\
\hline C99 & - C100 & - H100 & $=119.90$ \\
\hline C102 & - C101 & - C100 & $=120.99(18)$ \\
\hline C102 & - C101 & - H101 & $=119.50$ \\
\hline C100 & - C101 & - H101 & $=119.50$ \\
\hline C101 & - C102 & - C103 & $=119.57(17)$ \\
\hline C101 & - C102 & - H102 & $=120.20$ \\
\hline C103 & - C102 & $-\mathrm{H} 102$ & $=120.20$ \\
\hline C104 & - C103 & - C102 & $=119.77(18)$ \\
\hline C104 & - C103 & - H103 & $=120.10$ \\
\hline C102 & - C103 & $-\mathrm{H} 103$ & $=120.10$ \\
\hline C103 & - C104 & - C99 & $=121.74(17)$ \\
\hline C103 & - C104 & - H104 & $=119.10$ \\
\hline C99 & $-\mathrm{C} 104$ & $-\mathrm{H} 104$ & $=119.10$ \\
\hline $\mathrm{Cl} 1$ & - C105 & $-\mathrm{Cl} 2=$ & $=111.22(13)$ \\
\hline $\mathrm{Cl} 1$ & - C105 & $-\mathrm{H} 10 \mathrm{C}$ & $=109.40$ \\
\hline $\mathrm{Cl} 2$ & - C105 & $-\mathrm{H} 10 \mathrm{C}$ & $=109.40$ \\
\hline $\mathrm{Cl} 1$ & - C105 & - H10D & $=109.40$ \\
\hline $\mathrm{Cl} 2$ & - C105 & - H10D & $=109.40$ \\
\hline $\mathrm{H} 10 \mathrm{C}$ & - C105 & - H10D & $=108.00$ \\
\hline
\end{tabular}


Table S10. Torsion angles $\left[{ }^{\circ}\right]$ for 4 aa.

$$
\begin{array}{lllll}
\mathrm{C} 13 & -\mathrm{P} 1 & -\mathrm{C} 1 & -\mathrm{C} 6 & =-179.31(16) \\
\mathrm{C} 7 & -\mathrm{P} 1 & -\mathrm{C} 1 & -\mathrm{C} 6 & =-75.61(17) \\
\mathrm{C} 13 & -\mathrm{P} 1 & -\mathrm{C} 1 & -\mathrm{C} 2 & =60.56(15) \\
\mathrm{C} 7 & -\mathrm{P} 1 & -\mathrm{C} 1 & -\mathrm{C} 2 & =164.26(13) \\
\mathrm{C} 6 & -\mathrm{C} 1 & -\mathrm{C} 2 & -\mathrm{C} 3 & =51.00(2) \\
\mathrm{P} 1 & -\mathrm{C} 1 & -\mathrm{C} 2 & -\mathrm{C} 3 & =170.93(15) \\
\mathrm{C} 1 & -\mathrm{C} 2 & -\mathrm{C} 3 & -\mathrm{C} 4 & =-54.50(3) \\
\mathrm{C} 2 & -\mathrm{C} 3 & -\mathrm{C} 4 & -\mathrm{C} 5 & =56.80(3) \\
\mathrm{C} 3 & -\mathrm{C} 4 & -\mathrm{C} 5 & -\mathrm{C} 6 & =-57.80(3) \\
\mathrm{C} 4 & -\mathrm{C} 5 & -\mathrm{C} 6 & -\mathrm{C} 1 & =56.50(3) \\
\mathrm{C} 2 & -\mathrm{C} 1 & -\mathrm{C} 6 & -\mathrm{C} 5 & =-51.70(3) \\
\mathrm{P} 1 & -\mathrm{C} 1 & -\mathrm{C} 6 & -\mathrm{C} 5 & =-170.65(19) \\
\mathrm{C} 13 & -\mathrm{P} 1 & -\mathrm{C} 7 & -\mathrm{C} 8 & =60.15(16) \\
\mathrm{C} 1 & -\mathrm{P} 1 & -\mathrm{C} 7 & -\mathrm{C} 8 & =-45.63(17) \\
\mathrm{C} 13 & -\mathrm{P} 1 & -\mathrm{C} 7 & -\mathrm{C} 12 & =-170.48(13) \\
\mathrm{C} 1 & -\mathrm{P} 1 & -\mathrm{C} 7 & -\mathrm{C} 12 & =83.75(15) \\
\mathrm{C} 12 & -\mathrm{C} 7 & -\mathrm{C} 8 & -\mathrm{C} 9 & =56.60(2) \\
\mathrm{P} 1 & -\mathrm{C} 7 & -\mathrm{C} 8 & -\mathrm{C} 9 & =-173.24(16) \\
\mathrm{C} 7 & -\mathrm{C} 8 & -\mathrm{C} 9 & -\mathrm{C} 10 & =-57.40(3) \\
\mathrm{C} 8 & -\mathrm{C} 9 & -\mathrm{C} 10 & -\mathrm{C} 11 & =56.00(3) \\
\mathrm{C} 9 & -\mathrm{C} 10 & -\mathrm{C} 11 & -\mathrm{C} 12 & =-54.80(3) \\
\mathrm{C} 10 & -\mathrm{C} 11 & -\mathrm{C} 12 & -\mathrm{C} 7 & =54.90(2) \\
\mathrm{C} 8 & -\mathrm{C} 7 & -\mathrm{C} 12 & -\mathrm{C} 11 & =-55.50(2) \\
\mathrm{P} 1 & -\mathrm{C} 7 & -\mathrm{C} 12 & -\mathrm{C} 11 & =170.96(14) \\
\mathrm{C} 7 & -\mathrm{P} 1 & -\mathrm{C} 13 & -\mathrm{C} 14 & =-77.35(18) \\
\mathrm{C} 1 & -\mathrm{P} 1 & -\mathrm{C} 13 & -\mathrm{C} 14 & =32.15(19) \\
\mathrm{C} 7 & -\mathrm{P} 1 & -\mathrm{C} 13 & -\mathrm{C} 18 & =103.04(15) \\
\mathrm{C} 1 & -\mathrm{P} 1 & -\mathrm{C} 13 & -\mathrm{C} 18 & =-147.45(15) \\
\mathrm{C} 18 & -\mathrm{C} 13 & -\mathrm{C} 14 & -\mathrm{C} 15 & =-1.20(3) \\
\mathrm{P} 1 & -\mathrm{C} 13 & -\mathrm{C} 14 & -\mathrm{C} 15 & =179.22(17) \\
\mathrm{C} 13 & -\mathrm{C} 14 & -\mathrm{C} 15 & -\mathrm{C} 16 & =2.80(3) \\
\mathrm{C} 18 & -\mathrm{C} 15 & -\mathrm{C} 16 & -\mathrm{C} 17 & =-1.30(3) \\
\mathrm{C} 19 & -\mathrm{C} 210 & -\mathrm{C} 21 & -\mathrm{C} 22 & =0.10(3) \\
\mathrm{C} 25 & -\mathrm{C} 20 & -\mathrm{C} 21 & -\mathrm{C} 22 & =-179.67(17) \\
\mathrm{C} 15 & -\mathrm{C} 16 & -\mathrm{C} 17 & -\mathrm{C} 18 & =-1.90(3) \\
\mathrm{C} 16 & -\mathrm{C} 17 & -\mathrm{C} 18 & -\mathrm{C} 13 & =3.50(3) \\
\mathrm{C} 16 & -\mathrm{C} 17 & -\mathrm{C} 18 & -\mathrm{C} 19 & =-175.34(16) \\
\mathrm{C} 14 & -\mathrm{C} 13 & -\mathrm{C} 18 & -\mathrm{C} 17 & =-2.00(3) \\
\mathrm{P} 1 & -\mathrm{C} 13 & -\mathrm{C} 18 & -\mathrm{C} 17 & =177.67(13) \\
\mathrm{C} 14 & -\mathrm{C} 13 & -\mathrm{C} 18 & -\mathrm{C} 19 & =176.87(17) \\
\mathrm{P} 1 & -\mathrm{C} 13 & -\mathrm{C} 18 & -\mathrm{C} 19 & =-3.50(2) \\
\mathrm{C} 17 & -\mathrm{C} 18 & -\mathrm{C} 19 & -\mathrm{C} 24 & =-96.76(19) \\
\mathrm{C} 13 & -\mathrm{C} 18 & -\mathrm{C} 19 & -\mathrm{C} 24 & =84.40(2) \\
\mathrm{C} 17 & -\mathrm{C} 18 & -\mathrm{C} 19 & -\mathrm{C} 20 & =86.70(2) \\
\mathrm{C} 13 & -\mathrm{C} 18 & -\mathrm{C} 19 & -\mathrm{C} 20 & =-92.10(2) \\
\mathrm{C} 19 & -\mathrm{C} 20 & -\mathrm{C} 21 & =-1.40(2) \\
\mathrm{C} & -\mathrm{C} 20 & -\mathrm{C} 25 & =175.16(15) \\
\mathrm{C} & -\mathrm{C} 19.38(16) \\
\mathrm{C} & & &
\end{array}
$$




\begin{tabular}{|c|c|c|c|c|}
\hline C20 & $-\mathrm{C} 21$ & $-\mathrm{C} 22$ & $-\mathrm{C} 23$ & $=1.10(3)$ \\
\hline C21 & $-C 22$ & $-\mathrm{C} 23$ & $-\mathrm{C} 24$ & $=-1.00(3)$ \\
\hline $\mathrm{C} 22$ & $-\mathrm{C} 23$ & $-\mathrm{C} 24$ & $-\mathrm{C} 19$ & $=-0.30(3)$ \\
\hline $\mathrm{C} 22$ & $-\mathrm{C} 23$ & $-\mathrm{C} 24$ & - C39 & $=-176.93(17)$ \\
\hline C20 & - C19 & $-\mathrm{C} 24$ & $-\mathrm{C} 23$ & $=1.50(2)$ \\
\hline C18 & - C19 & $-\mathrm{C} 24$ & $-\mathrm{C} 23$ & $=-175.12(16)$ \\
\hline C20 & $-\mathrm{C} 19$ & $-\mathrm{C} 24$ & - C39 & $=177.92(15)$ \\
\hline C18 & - C19 & $-C 24$ & - C39 & $=1.30(2)$ \\
\hline C21 & $-\mathrm{C} 20$ & $-\mathrm{C} 25$ & $-\mathrm{C} 32$ & $=-45.10(2)$ \\
\hline C19 & $-\mathrm{C} 20$ & $-\mathrm{C} 25$ & $-\mathrm{C} 32$ & $=135.13(18)$ \\
\hline C21 & $-\mathrm{C} 20$ & $-\mathrm{C} 25$ & $-\mathrm{C} 26$ & $=128.41(17)$ \\
\hline C19 & $-\mathrm{C} 20$ & $-\mathrm{C} 25$ & $-\mathrm{C} 26$ & $=-51.40(2)$ \\
\hline C32 & $-\mathrm{C} 25$ & $-\mathrm{C} 26$ & $-\mathrm{C} 27$ & $=125.64(19)$ \\
\hline $\mathrm{C} 20$ & $-\mathrm{C} 25$ & $-\mathrm{C} 26$ & $-\mathrm{C} 27$ & $=-47.80(2)$ \\
\hline C32 & $-\mathrm{C} 25$ & $-\mathrm{C} 26$ & $-\mathrm{C} 31$ & $=-51.90(2)$ \\
\hline C20 & $-\mathrm{C} 25$ & $-\mathrm{C} 26$ & $-\mathrm{C} 31$ & $=134.71(17)$ \\
\hline C31 & $-\mathrm{C} 26$ & $-\mathrm{C} 27$ & $-\mathrm{C} 28$ & $=1.20(3)$ \\
\hline $\mathrm{C} 25$ & $-\mathrm{C} 26$ & $-\mathrm{C} 27$ & $-\mathrm{C} 28$ & $=-176.32(17)$ \\
\hline C26 & $-\mathrm{C} 27$ & $-\mathrm{C} 28$ & $-\mathrm{C} 29$ & $=0.20(3)$ \\
\hline C27 & $-\mathrm{C} 28$ & $-\mathrm{C} 29$ & $-\mathrm{C} 30$ & $=-1.00(3)$ \\
\hline C28 & $-\mathrm{C} 29$ & $-\mathrm{C} 30$ & $-\mathrm{C} 31$ & $=0.40(3)$ \\
\hline C29 & - C30 & $-\mathrm{C} 31$ & $-\mathrm{C} 26$ & $=1.10(3)$ \\
\hline C27 & $-\mathrm{C} 26$ & $-\mathrm{C} 31$ & - C30 & $=-1.80(3)$ \\
\hline C25 & $-\mathrm{C} 26$ & $-\mathrm{C} 31$ & $-\mathrm{C} 30$ & $=175.69(17)$ \\
\hline C26 & $-\mathrm{C} 25$ & $-\mathrm{C} 32$ & $-\mathrm{C} 33$ & $=-8.50(3)$ \\
\hline C20 & $-\mathrm{C} 25$ & $-\mathrm{C} 32$ & $-\mathrm{C} 33$ & $=164.89(17)$ \\
\hline C25 & $-\mathrm{C} 32$ & $-\mathrm{C} 33$ & $-\mathrm{C} 38$ & $=152.06(19)$ \\
\hline C25 & $-\mathrm{C} 32$ & $-\mathrm{C} 33$ & $-\mathrm{C} 34$ & $=-33.40(3)$ \\
\hline C38 & $-\mathrm{C} 33$ & $-\mathrm{C} 34$ & $-\mathrm{C} 35$ & $=-1.00(3)$ \\
\hline C32 & $-\mathrm{C} 33$ & $-\mathrm{C} 34$ & - C35 & $=-175.71(17)$ \\
\hline C33 & $-\mathrm{C} 34$ & $-\mathrm{C} 35$ & $-\mathrm{C} 36$ & $=0.70(3)$ \\
\hline C34 & $-\mathrm{C} 35$ & $-\mathrm{C} 36$ & $-\mathrm{C} 37$ & $=0.00(3)$ \\
\hline C35 & $-\mathrm{C} 36$ & $-\mathrm{C} 37$ & $-\mathrm{C} 38$ & $=-0.20(3)$ \\
\hline C36 & $-\mathrm{C} 37$ & $-\mathrm{C} 38$ & $-\mathrm{C} 33$ & $=-0.10(3)$ \\
\hline C34 & $-\mathrm{C} 33$ & $-\mathrm{C} 38$ & $-\mathrm{C} 37$ & $=0.80(3)$ \\
\hline C32 & $-\mathrm{C} 33$ & $-\mathrm{C} 38$ & $-\mathrm{C} 37$ & $=175.60(17)$ \\
\hline $\mathrm{C} 23$ & $-\mathrm{C} 24$ & - C39 & - C46 & $=51.00(2)$ \\
\hline C19 & $-\mathrm{C} 24$ & - C39 & $-\mathrm{C} 46$ & $=-125.54(18)$ \\
\hline C23 & $-\mathrm{C} 24$ & - C39 & $-\mathrm{C} 40$ & $=-124.11(18)$ \\
\hline C19 & $-\mathrm{C} 24$ & - C39 & $-\mathrm{C} 40$ & $=59.30(2)$ \\
\hline C46 & - C39 & $-\mathrm{C} 40$ & $-\mathrm{C} 45$ & $=56.60(2)$ \\
\hline C24 & - C39 & $-\mathrm{C} 40$ & $-\mathrm{C} 45$ & $=-128.43(18)$ \\
\hline C46 & $-\mathrm{C} 39$ & $-\mathrm{C} 40$ & - C41 & $=-117.30(2)$ \\
\hline C24 & - C39 & $-\mathrm{C} 40$ & $-\mathrm{C} 41$ & $=57.70(2)$ \\
\hline C45 & $-\mathrm{C} 40$ & - C41 & $-\mathrm{C} 42$ & $=-1.40(3)$ \\
\hline C39 & $-\mathrm{C} 40$ & $-\mathrm{C} 41$ & $-\mathrm{C} 42$ & $=172.64(17)$ \\
\hline $\mathrm{C} 40$ & - C41 & $-\mathrm{C} 42$ & $-\mathrm{C} 43$ & $=-0.10(3)$ \\
\hline C41 & - C42 & $-\mathrm{C} 43$ & - C44 & $=1.20(3)$ \\
\hline C42 & $-\mathrm{C} 43$ & $-\mathrm{C} 44$ & $-\mathrm{C} 45$ & $=-0.80(3)$ \\
\hline C43 & - C44 & - C45 & $-\mathrm{C} 40$ & $=-0.70(3)$ \\
\hline C41 & $-\mathrm{C} 40$ & $-\mathrm{C} 45$ & - C44 & $=1.80(3)$ \\
\hline C39 & $-\mathrm{C} 40$ & $-\mathrm{C} 45$ & $-\mathrm{C} 44$ & $=-172.22(17)$ \\
\hline
\end{tabular}




\begin{tabular}{|c|c|c|c|c|}
\hline & - C39 & - C46 & - C47 & \\
\hline 24 & - C39 & - C46 & $-\mathrm{C} 47$ & $=-172.17(17)$ \\
\hline 39 & C46 & $-\mathrm{C} 47$ & - C52 & $=-150.53(19)$ \\
\hline & C46 & - C47 & $-\mathrm{C} 48$ & $=31.50(3)$ \\
\hline & C47 & $-\mathrm{C} 48$ & $-C 49$ & $=2.50(3)$ \\
\hline & C47 & $-\mathrm{C} 48$ & $-C 49$ & $=-179.53(17$ \\
\hline 17 & - C48 & - C49 & - C50 & $=-0.40(3)$ \\
\hline 8 & - C49 & - C50 & - C51 & $=-1.50(3)$ \\
\hline 9 & - C50 & - C51 & - C52 & $=1.10$ \\
\hline & C51 & - C52 & $-\mathrm{C} 47$ & $=1.1$ \\
\hline & $-\mathrm{C} 47$ & - C52 & - C51 & $=-2.8$ \\
\hline 46 & $-\mathrm{C} 47$ & $-\mathrm{C} 52$ & - C51 & $=179$. \\
\hline 55 & - P2 & - C53 & - C54 & $=170.2$ \\
\hline & - P2 & & & $=66.2$ \\
\hline & $-P 2$ & & & $--\infty 0$. \\
\hline & - P2 & C53 & C58 & $=-172$. \\
\hline 88 & - C53 & - C54 & - C55 & $=54$. \\
\hline 2 & $-\mathrm{C53}$ & $-\mathrm{C} 54$ & $-\mathrm{C} 55$ & $=174$. \\
\hline 53 & $-\mathrm{C} 54$ & - C55 & 56 & -56 \\
\hline & & & & $=56$. \\
\hline & 56 & Cs/ & 58 & $=-54$ \\
\hline & $-\mathrm{C} 57$ & C58 & C53 & $=54$. \\
\hline 4 & - C53 & $-C 58$ & $-\mathrm{C} 57$ & $=-53$. \\
\hline & - C53 & - C58 & - C57 & $=-175.2$ \\
\hline & $-P$ & & & $=-69.7$ \\
\hline & & & & $=35$. \\
\hline & - P2 & & & $=161$ \\
\hline 3 & $-P 2$ & C59 & c64 & $=-93$. \\
\hline 54 & - C59 & - C60 & - C61 & 5 \\
\hline & 9 & $-\mathrm{C}$ & & $=177$. \\
\hline & & & 662 & $=55$. \\
\hline & & & & $=-56$ \\
\hline & C62 & C63 & & $=5$ \\
\hline 52 & $-\mathrm{C} 63$ & - C64 & C59 & $=-55$ \\
\hline & & & & $=53$. \\
\hline & & & & $=-173$. \\
\hline & & & & $=-36$ \\
\hline & - & 5 & & $=71$. \\
\hline & רח & & & $=146$ \\
\hline & & & & $=-105$. \\
\hline & & & - C67 & $=-4.3$ \\
\hline & & & & $=1 /$ \\
\hline & & & & \\
\hline P? & - C65 & - C66 & $-\mathrm{C} 71$ & $=-10.8$ \\
\hline 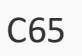 & - C66 & - C67 & - C68 & $=3.8$ \\
\hline & $-\mathrm{C} 66$ & $-\mathrm{C} 67$ & $-\mathrm{C} 68$ & $=-172$. \\
\hline & $-\mathrm{C} 67$ & $-\mathrm{C} 68$ & $-C 69$ & $=-0.4$ \\
\hline & & & & $=-2.4$ \\
\hline & & & & $=1.80$ \\
\hline & $-\mathrm{C} 65$ & $-\mathrm{C} 70$ & $-C 69$ & $=1.70(3)$ \\
\hline P2 & $-\mathrm{C65}$ & - C70 & - C69 & $=-175.66(15)$ \\
\hline C67 & - C66 & $-\mathrm{C} 71$ & $-\mathrm{C} 72$ & $=-91.65(19)$ \\
\hline
\end{tabular}




\begin{tabular}{|c|c|c|c|c|}
\hline 65 & - C66 & - C71 & $-\mathrm{C} 72$ & $=92.00(2)$ \\
\hline 67 & - C66 & - C71 & $-\mathrm{C} 76$ & $=86.03(19)$ \\
\hline & C66 & - C71 & - C76 & \\
\hline & C71 & $-\mathrm{C} 72$ & - C73 & $=-3.40(2)$ \\
\hline 6 & $-\mathrm{C} 71$ & - C72 & $-\mathrm{C} 73$ & $=174.32(15)$ \\
\hline 6 & - C71 & - C72 & - C91 & $=172.73(15)$ \\
\hline 6 & - C71 & - C72 & - C91 & $=-9.60(2)$ \\
\hline & $-\mathrm{C} 72$ & - C73 & $-\mathrm{C} 74$ & $=0.80(3)$ \\
\hline & $-\mathrm{C} 72$ & - C73 & - C74 & $=-175$. \\
\hline 72 & $-\mathrm{C} 73$ & - C74 & $-C 75$ & $=1.90$ \\
\hline 73 & - C74 & - C75 & - C76 & $=-2.0 c$ \\
\hline 4 & - C75 & - C76 & - C71 & $=-0.7$ \\
\hline 4 & $-C 75$ & - C76 & - C77 & -178 \\
\hline 72 & - C71 & - C76 & $-\mathrm{C} 75$ & $=3.3$ \\
\hline 56 & $-\mathrm{C} 71$ & $-\mathrm{C} 76$ & $-\mathrm{C} 75$ & $=-174$ \\
\hline 72 & $-\mathrm{C} 71$ & $-C 76$ & - C77 & $=-17$ \\
\hline 56 & - C71 & - C76 & $-\mathrm{C} 77$ & $=8.4$ \\
\hline 5 & - C76 & - C77 & 84 & ב \\
\hline 71 & $-\mathrm{C} 76$ & C77 & C84 & $=11$ \\
\hline 75 & $-\mathrm{C} 76$ & $-\mathrm{C} 77$ & $-\mathrm{C} 78$ & $=119$ \\
\hline 1 & $-\mathrm{C} 76$ & $-\mathrm{C} 77$ & $-\mathrm{C} 78$ & $=-63$. \\
\hline 34 & $-\mathrm{C} 77$ & $-\mathrm{C} 78$ & - C79 & $=-52$. \\
\hline 76 & $-\mathrm{C} 77$ & & C79 & \\
\hline & $-\mathrm{C} 77$ & $-\mathrm{C} 78$ & $-\mathrm{C} 83$ & $=129$ \\
\hline 76 & $-\mathrm{C} 77$ & $-\mathrm{C} 78$ & $-\mathrm{C} 83$ & $=-47$ \\
\hline 33 & $-\mathrm{C} 78$ & - C79 & $-\mathrm{C} 80$ & $=-1$. \\
\hline 77 & $-\mathrm{C} 78$ & $-C 79$ & $-\mathrm{C} 80$ & $=-17$ \\
\hline 78 & - C79 & $-\mathrm{C} 80$ & - C81 & -0 \\
\hline & $-\mathrm{C} 80$ & & $-\mathrm{C} 82$ & \\
\hline 80 & $-\mathrm{C} 81$ & & $\mathrm{C} 83$ & $=-0$. \\
\hline 81 & $-\mathrm{C} 82$ & $-\mathrm{C} 83$ & - C78 & $=-0$. \\
\hline 79 & $-\mathrm{C} 78$ & $-\mathrm{C} 83$ & $-\mathrm{C} 82$ & $=18$ \\
\hline 77 & $-\mathrm{C} 78$ & $-\mathrm{C} 83$ & $-\mathrm{C} 82$ & $=179$ \\
\hline 70 & - C77 & cen & $-\mathrm{C} 85$ & \\
\hline 76 & $-\mathrm{C} 77$ & & & $=174$ \\
\hline 77 & 84 & & & $=1$. \\
\hline 77 & $\mathrm{C} 84$ & 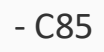 & C90 & $=-28$ \\
\hline 90 & $-\mathrm{C} 85$ & $-\mathrm{C} 86$ & $-\mathrm{C} 87$ & -2 \\
\hline 84 & $-\mathrm{C} 85$ & & & \\
\hline 85 & $-\mathrm{C} 86$ & $-\mathrm{C} 87$ & $-\mathrm{C} 88$ & $=-1$. \\
\hline 86 & 87 & & $-C 89$ & $=-0$ \\
\hline 27 & C88 & & $\mathrm{C9O}$ & $=1$ \\
\hline C88 & $-C 89$ & $-C 9$ & $-\mathrm{C} 85$ & $=0.6$ \\
\hline C86 & $-\mathrm{C} 85$ & - C90 & $-C 89$ & $=-2.7$ \\
\hline & $-\mathrm{C} 85$ & & $-\mathrm{C} 89$ & $=-11$ \\
\hline C73 & $-\mathrm{C} 72$ & & - C98 & \\
\hline C71 & & & & $=-12$ \\
\hline & $-\mathrm{C} 72$ & & - C92 & --121 \\
\hline C71 & $-\mathrm{C} 72$ & - C91 & - C92 & $=62.60(2)$ \\
\hline C98 & - C91 & - C92 & - C97 & $=57.30(3)$ \\
\hline C7. & - C91 & - C92 & - C97 & $=-128.70(18$ \\
\hline C9ع & - C91 & - C92 & - C93 & $=-125.40(2)$ \\
\hline
\end{tabular}




\begin{tabular}{|c|c|c|c|c|}
\hline $\mathrm{C} 72$ & - C91 & - C92 & - C93 & $=48.50(2)$ \\
\hline C97 & - C92 & - C93 & - C94 & $=0.00(3)$ \\
\hline C91 & - C92 & $-\mathrm{C93}$ & - C94 & $=-177.30(18)$ \\
\hline C92 & - C93 & - C94 & - C95 & $=0.10(3)$ \\
\hline C93 & - C94 & - C95 & - C96 & $=0.10(3)$ \\
\hline C94 & - C95 & - C96 & - C97 & $=-0.40(3)$ \\
\hline C95 & - C96 & - C97 & - C92 & $=0.50(3)$ \\
\hline C93 & - C92 & - C97 & - C96 & $=-0.30(3)$ \\
\hline C91 & - C92 & - C97 & - C96 & $=176.91(18)$ \\
\hline C92 & - C91 & - C98 & - C99 & $=7.00(3)$ \\
\hline C72 & - C91 & - C98 & - C99 & $=-167.07(17)$ \\
\hline C91 & - C98 & - C99 & - C100 & $=-4.40(3)$ \\
\hline C91 & - C98 & - C99 & - C104 & $=171.66(19)$ \\
\hline C104 & - C99 & $-\mathrm{C} 100$ & - C101 & $=0.20(3)$ \\
\hline C98 & - C99 & - C100 & - C101 & $=176.09(17)$ \\
\hline C99 & - C100 & $-\mathrm{C} 101$ & - C102 & $=-0.70(3)$ \\
\hline C100 & - C101 & - C102 & - C103 & $=0.40(3)$ \\
\hline C101 & - C102 & - C103 & - C104 & $=0.60(3)$ \\
\hline C102 & - C103 & - C104 & - C99 & $=-1.10(3)$ \\
\hline C100 & - C99 & $-\mathrm{C} 104$ & - C103 & $=0.80(3)$ \\
\hline C98 & - C99 & - C104 & - C103 & $=-175.64(16)$ \\
\hline
\end{tabular}

\section{Crystal measurement for 5a:}

Crystallization by diffusion method from $\mathrm{CH}_{2} \mathrm{Cl}_{2}$ solution with $n$-hexane has afforded single crystal to analyze by X-ray diffraction.

The single crystal X-ray diffraction studies were carried out on D8 VENTURE Bruker AXS diffractometer equipped with a (CMOS) PHOTON 100 detector $\left[{ }^{*}\right]$, Mo-K $\alpha$ radiation $(\lambda=0.71073$, multilayer monochromator) , $T=150 \mathrm{~K}$; triclinic $P$-1 (I.T.\#2), $a=11.1775(11), b=14.2939(14), c=15.4307(16), \alpha$ $=90.257(3), b=97.380(4), v=91.255(4), V=2444.3(4)^{3} . Z=1, d=1.432 \mathrm{~g} . \mathrm{cm}^{-3}, \mu=0.777 \mathrm{~mm}^{-1}$. The structure was solved by dual-space algorithm using the SHELXT program [1], and then refined with fullmatrix least-squares methods based on $F^{2}$ (SHELXL) [2]. All non-hydrogen atoms were refined with anisotropic atomic displacement parameters. $\mathrm{H}$ atoms were finally included in their calculated positions and treated as riding on their parent atom with constrained thermal parameters. A final refinement on $F^{2}$ with 11239 unique intensities and 559 parameters converged at $\omega R_{F}^{2}=0.1633\left(R_{F}=\right.$ 0.0647 ) for 7972 observed reflections with $I>2 \sigma(I)$.

[1] G. M. Sheldrick, Acta Cryst. A71 (2015) 3-8

[2] Sheldrick G.M., Acta Cryst. C71 (2015) 3-8 


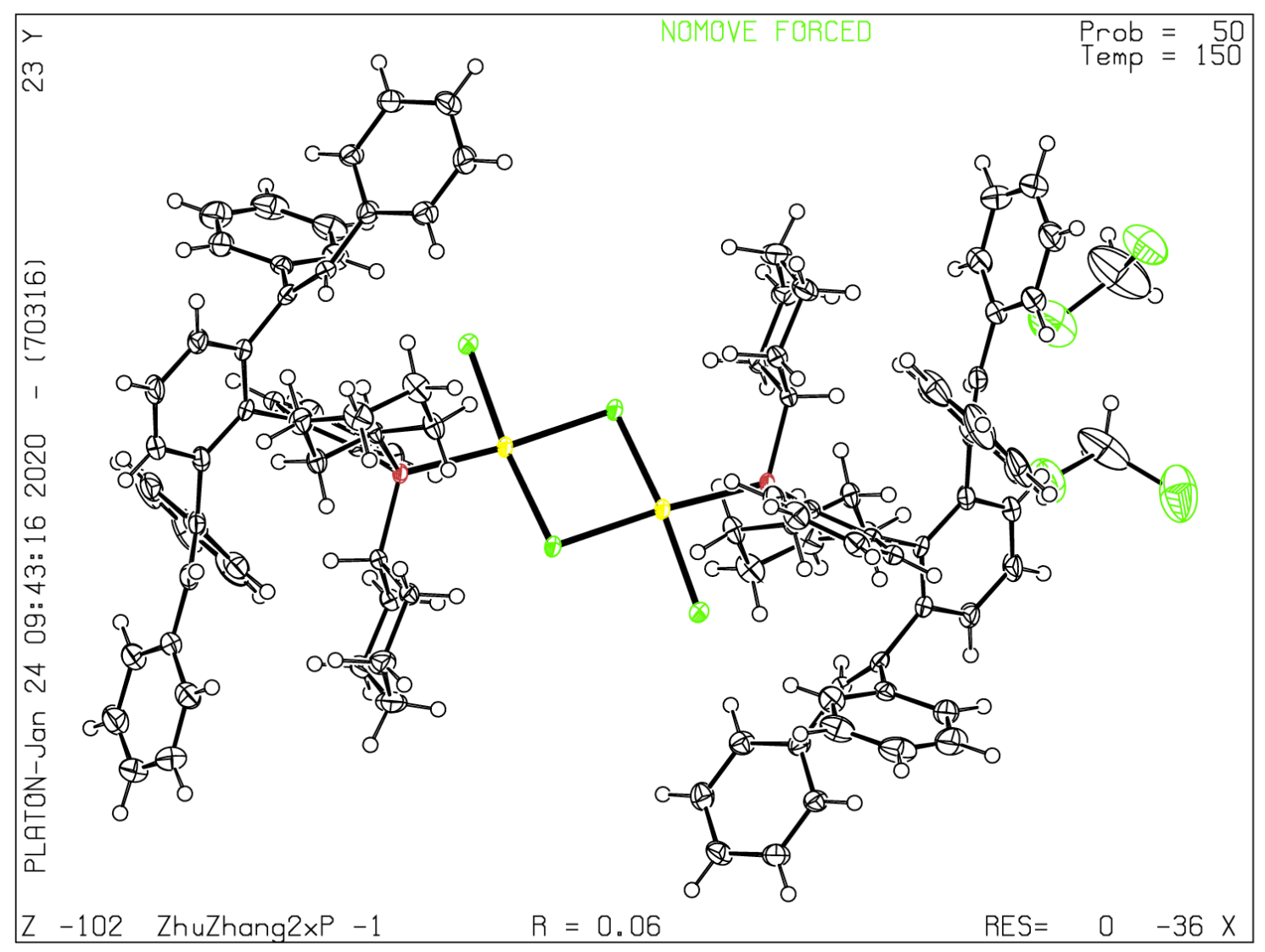

Figure S3. ORTEP diagram of the solid-state molecular structure of $\mathbf{5 a}$. Thermal ellipsoids are at the 50\% probability level. (CCDC 1982983).

Table S11. Crystal data and structure refinement for $\mathbf{5 0 .}$

Empirical formula

Formula weight

Temperature

Wavelength

Crystal system, space group

Unit cell dimensions

Volume

$Z$, Calculated density

Absorption coefficient

$F(000)$

Crystal size

Crystal color

Crystal description

Theta range for data collection

$(\sin \theta / \lambda)_{\max }\left(\AA^{-1}\right)$

h_min, h_max
$\mathrm{C}_{108} \mathrm{H}_{110} \mathrm{Cl}_{12} \mathrm{P}_{2} \mathrm{Pd}_{2}$

$2108.09 \mathrm{~g} / \mathrm{mol}$

$150 \mathrm{~K}$

$0.71073 \AA$

triclinic, $P-1$

$a=11.1775(11) \AA$

$b=14.2939(14) \AA$

$\mathrm{c}=15.4307(16) \AA$

$\alpha=90.257(3)^{\circ}$

$\beta=97.380(4)^{\circ}$

$\gamma=91.255(4)^{\circ}$

2444.3(4) $\AA^{3}$

$1,1.432 \mathrm{~g} \cdot \mathrm{cm}^{-3}$

$0.777 \mathrm{~mm}^{-1}$

1084

$0.330 \times 0.220 \times 0.130 \mathrm{~mm}$

orange

pism

2.300 to $27.588^{\circ}$

0.652

$-14,14$ 
k_min, k_max

I min, I max

Reflections collected / unique

Reflections [ $1>2 \sigma]$

Completeness to theta_max

Absorption correction type

Max. and min. transmission

Refinement method

Data / restraints / parameters

${ }^{b} \mathrm{~S}$ (Goodness-of-fit)

Final $R$ indices $[\mid>2 \sigma]$

$R$ indices (all data)

Largest diff. peak and hole
$-18,18$

$-20,20$

$40084 / 11239\left[R(\text { int })^{a}=0.0838\right]$

7972

0.991

multi-scan

$0.904,0.771$

Full-matrix least-squares on $F^{2}$

11239 / 0 / 559

1.005

$R 1^{c}=0.0647, w R 2^{d}=0.1633$

$R 1^{c}=0.1025, w R 2^{d}=0.1904$

1.809 and $-1.631 \mathrm{e}^{-} . \AA^{-3}$

Table S11. Fractional atomic coordinates, site occupancy (\%) and equivalent isotropic displacement parameters $\left(\AA^{2}\right)$ for 5 a. $U(e q)$ is defined as one third of the trace of the orthogonalized Uij tensor.

\begin{tabular}{|c|c|c|c|c|c|}
\hline Atom & $\mathrm{x}$ & y & z & occ. & $\mathrm{U}(\mathrm{eq}) \quad \mathrm{adp}$ \\
\hline Pd1 & $0.43031(3)$ & $0.93359(2)$ & 2) $0.07514(2)$ & 1 & $0.01642(12)$ Uani \\
\hline $\mathrm{Cl} 1$ & $0.58505(11)$ & ) $0.91291(8)$ & 3) $-0.00867(8)$ & 1 & $0.0214(3)$ Uani \\
\hline $\mathrm{Cl} 2$ & $0.26966(11)$ & $0.96535(9)$ & $0.14673(8)$ & 1 & 0.0249(3) Uani \\
\hline P1 & $0.43226(11)$ & ) $0.79354(8)$ & 3) $0.14286(7)$ & 1 & $0.0141(2)$ Uani \\
\hline $\mathrm{C} 1$ & $0.5575(4)$ & $0.7142(3)$ & $0.1322(3)$ & 1 & 0.0188(10) Uani \\
\hline $\mathrm{H} 1$ & 0.540859 & 0.656989 & 0.166205 & 1 & 0.023 Uiso \\
\hline $\mathrm{C} 2$ & $0.5658(5)$ & $0.6800(3)$ & $0.0385(3)$ & 1 & $0.0223(10)$ Uani \\
\hline $\mathrm{H} 2 \mathrm{~A}$ & 0.486699 & 0.653102 & 0.012758 & 1 & $0.027 \quad$ Uiso \\
\hline $\mathrm{H} 2 \mathrm{~B}$ & 0.585626 & 0.733680 & 0.002176 & 1 & 0.027 Uiso \\
\hline C3 & $0.6626(5)$ & $0.6060(4)$ & $0.0381(4)$ & 1 & $0.0337(13)$ Uani \\
\hline $\mathrm{H} 3 \mathrm{~A}$ & 0.638113 & 0.549615 & 0.069107 & 1 & 0.040 Uiso \\
\hline H3B & 0.669512 & 0.587622 & -0.022991 & 1 & $0.040 \quad$ Uiso \\
\hline C4 & $0.7852(5)$ & $0.6422(4)$ & $0.0820(5)$ & 1 & 0.0404(15) Uani \\
\hline $\mathrm{H} 4 \mathrm{~A}$ & 0.814636 & 0.693588 & 0.046897 & 1 & $0.048 \quad$ Uiso \\
\hline H4B & 0.844064 & 0.591254 & 0.084746 & 1 & $0.048 \quad$ Uiso \\
\hline C5 & $0.7757(5)$ & $0.6779(4)$ & $0.1743(4)$ & 1 & $0.0326(13)$ Uani \\
\hline H5A & 0.754208 & 0.624977 & 0.210920 & 1 & 0.039 Uiso \\
\hline H5B & 0.855099 & 0.703945 & 0.200385 & 1 & 0.039 Uiso \\
\hline C6 & $0.6813(5)$ & $0.7529(4)$ & $0.1739(4)$ & 1 & $0.0241(11)$ Uani \\
\hline $\mathrm{H} 6 \mathrm{~A}$ & 0.705167 & 0.807626 & 0.140410 & 1 & 0.029 Uiso \\
\hline H6B & 0.676063 & 0.773688 & 0.234517 & 1 & 0.029 \\
\hline C7 & $0.2906(4)$ & $0.7301(3)$ & $0.0990(3)$ & 1 & $0.0171(9) \quad$ Uani \\
\hline $\mathrm{H} 7$ & 0.226912 & 0.752861 & 0.133376 & 1 & 0.021 Uiso \\
\hline $\mathrm{C} 8$ & $0.2511(5)$ & $0.7585(4)$ & $0.0032(3)$ & 1 & 0.0246(11) Uani \\
\hline $\mathrm{H} 8 \mathrm{~A}$ & 0.245919 & 0.827476 & -0.000028 & 1 & $0.030 \quad$ Uiso \\
\hline H8B & 0.313000 & 0.738930 & -0.033252 & 1 & 0.030 \\
\hline C9 & $0.1302(5)$ & $0.7148(4)$ & $-0.0333(4)$ & 1 & $0.0312(12)$ Uani \\
\hline H9A & 0.111657 & 0.731276 & -0.095802 & 1 & $0.037 \quad$ Uiso \\
\hline H9B & 0.066369 & 0.740622 & -0.001879 & 1 & 0.037 \\
\hline C10 & $0.1298(5)$ & $0.6081(4)$ & $-0.0245(4)$ & 1 & $0.0321(13)$ Uani \\
\hline $\mathrm{H} 10 \mathrm{~A}$ & 0.048095 & 0.582181 & -0.044998 & 1 & 0.039 Uiso \\
\hline $\mathrm{H} 10 \mathrm{~B}$ & 0.186825 & 0.581276 & -0.061386 & 1 & 0.039 \\
\hline
\end{tabular}




\begin{tabular}{|c|c|c|c|c|c|}
\hline C11 & $0.1661(5)$ & $0.5809(4)$ & $0.0704(4)$ & 1 & 0.0266(11) Uani \\
\hline $\mathrm{H} 11 \mathrm{~A}$ & 0.168617 & 0.511866 & 0.074665 & 1 & 0.032 Uiso \\
\hline 11B & 0.104671 & 0.602862 & 0.106174 & 1 & 0.032 \\
\hline 12 & $0.2891(5)$ & $0.6227(3)$ & $0.1068(3)$ & 1 & $0.0220(10)$ Uani \\
\hline $2 \mathrm{~A}$ & 0.351824 & 0.596696 & 0.074422 & 1 & $0.026 \quad$ Uiso \\
\hline $12 B$ & 0.308236 & 0.605335 & 0.169016 & 1 & 0.026 \\
\hline 13 & $0.4471(4)$ & $0.8209(3)$ & $0.2609(3)$ & 1 & $0.0157(9)$ Uani \\
\hline 14 & $0.5236(5)$ & $0.8997(4)$ & $0.2827(3)$ & 1 & 0.0239(11) Uani \\
\hline 14 & 0.559052 & 0.930652 & 0.237634 & 1 & 0.029 Uiso \\
\hline 15 & $0.5485(5)$ & $0.9333(4)$ & $0.3671(4)$ & 1 & 0.0271(11) Uan \\
\hline 15 & 0.602894 & 0.984942 & 0.379 & 1 & 0.033 Uiso \\
\hline 16 & $0.4940(5)$ & $0.8914(4)$ & $0.4332(3)$ & 1 & $0.0263(11)$ Uan \\
\hline 16 & 0.508704 & 0.914935 & 0.491307 & 1 & $0.032 \quad$ Uiso \\
\hline 17 & $0.4183(4)$ & $0.8153(3)$ & 0.4131 & 1 & $0.0202(10)$ Uani \\
\hline 17 & 0.380001 & 0.787420 & 0.458 & 1 & $0.024 \quad$ Uiso \\
\hline 18 & $0.3950(4)$ & $0.7767(3)$ & 0.328 & 1 & 0.0159(9) Uani \\
\hline 19 & $0.3185(4)$ & $0.6885(3)$ & 0.320 & 1 & $0.0187(10)$ Uani \\
\hline 20 & $0.3726(5)$ & $0.6002(4)$ & 0.332 & 1 & $0.0221(10)$ Uani \\
\hline 21 & $0.2981(5)$ & $0.5201(4)$ & 0.325 & 1 & $0.0289(12)$ Uan \\
\hline H21 & 0.333655 & 0.460425 & 0.333 & 1 & 0.035 Uiso \\
\hline$C 22$ & $0.1744(5)$ & $0.5253(4)$ & 0.307 & 1 & $0.0307(12)$ Uan \\
\hline $\mathrm{H} 22$ & 0.125760 & 0.469743 & 0.30 & 1 & $0.037 \quad$ Uiso \\
\hline $\mathrm{C} 23$ & $0.1215(5)$ & $0.6115(4)$ & 0.297 & 1 & $0.0265(11)$ Uani \\
\hline 123 & 0.036229 & 0.614823 & 0.28 & 1 & 0.032 Uiso \\
\hline $\mathrm{C} 24$ & $0.1917(5)$ & $0.6940(4)$ & $0.304 \varepsilon$ & 1 & $0.0209(10)$ Uani \\
\hline$C 25$ & $0.5056(5)$ & $0.5857(4)$ & 0.353 & 1 & $0.0227(10)$ Uani \\
\hline$=26$ & $0.5569(5)$ & $0.5176(4)$ & 0.311 & 1 & $0.0246(11)$ Uan \\
\hline H26 & 0.508549 & 0.492106 & 0.26 & 1 & 0.030 Uiso \\
\hline$=27$ & $0.6794(5)$ & $0.4764(4)$ & 0.331 & 1 & $0.0288(12)$ Uani \\
\hline $\mathrm{C} 28$ & $0.7298(6)$ & $0.4331(4)$ & 0.264 & 1 & $0.0373(14)$ Uani \\
\hline †28 & 0.686899 & 0.432 & 0.20 & 1 & 0.045 Uiso \\
\hline$C 29$ & $0.8411(7)$ & $0.3907(5)$ & 0.279 & 1 & $0.0506(19)$ Uan \\
\hline 29 & 0.87 & 0.364 & 0.23 & 1 & 0.061 Uiso \\
\hline C30 & $0.9011(6)$ & $0.3879(5)$ & 0.363 & 1 & 0.0487(18) Uani \\
\hline 130 & 0.975872 & 0.356991 & 0.37 & 1 & $0.058 \quad$ Uiso \\
\hline C31 & $0.8526(6)$ & $0.4296(5)$ & 0.4313 & 1 & 0.0434(16) Uani \\
\hline H31 & 0.894870 & 0.428558 & 0.48 & 1 & 0.052 Uiso \\
\hline C32 & $0.7418(5)$ & $0.4734(4)$ & 0.415 & 1 & 0.0333(13) Uani \\
\hline H32 & 0.708424 & 0.501340 & 0.463 & 1 & 0.040 Uiso \\
\hline$=33$ & $0.5747(5)$ & $0.6404(4)$ & 0.425 & 1 & $0.0253(11)$ Uani \\
\hline C34 & $0.6819(5)$ & $0.6886(4)$ & 0.415 & 1 & $0.0315(12)$ Uani \\
\hline †34 & 0.71 & 0.6887 & 0.36 & 1 & 0.038 \\
\hline C35 & $0.7467(7)$ & $0.7362(4)$ & 0.4858 & 1 & $0.0483(18)$ Uan \\
\hline H35 & 0.819938 & 0.768364 & 0.478397 & 1 & $0.058 \quad$ Uiso \\
\hline C36 & $0.7047(8)$ & $0.7370(5)$ & $0.5669(5)$ & 1 & $0.054(2)$ \\
\hline H36 & 0.749595 & 0.769325 & 0.614 & 1 & 0.065 \\
\hline C37 & $0.5988(8)$ & $0.6914(5)$ & 0.5776 & 1 & $0.0487(19)$ Uani \\
\hline H37 & 0.569423 & 0.692855 & 0.632 & 1 & $0.058 \quad$ Uiso \\
\hline C38 & $0.5344(6)$ & $0.6428(4)$ & $0.5080(4)$ & 1 & $0.0338(13)$ Uani \\
\hline H38 & 0.461550 & 0.610580 & 0.516237 & 1 & 0.041 Uiso \\
\hline C39 & $0.1280(4)$ & $0.7855(4)$ & $0.2956(3)$ & 1 & $0.0205(10)$ Uan \\
\hline C40 & $0.0552(4)$ & $0.8031(4)$ & $0.2217(3)$ & 1 & $0.0205(10) \mathrm{Ua}$ \\
\hline
\end{tabular}




\begin{tabular}{|c|c|c|c|c|c|}
\hline $\mathrm{H} 40$ & 0.056219 & 0.758075 & 0.176321 & 1 & 0.025 \\
\hline C41 & $-0.0266(5)$ & $0.8821(4)$ & $0.2002(3)$ & 1 & $0.0228(10)$ Uani \\
\hline C42 & $-0.0450(5)$ & $0.9130(4)$ & $0.1137(3)$ & 1 & $0.0263(11)$ Uani \\
\hline $\mathrm{H} 42$ & -0.002936 & 0.884672 & 0.071120 & 1 & 0.032 Uiso \\
\hline C43 & $-0.1235(5)$ & $0.9841(4)$ & $0.0896(4)$ & 1 & 0.0321(13) Uani \\
\hline $\mathrm{H} 43$ & -0.132487 & 1.005937 & 0.031152 & 1 & $0.039 \quad$ Uiso \\
\hline C44 & $-0.1892(5)$ & $1.0240(4)$ & $0.1496(4)$ & 1 & $0.0320(13)$ Uani \\
\hline $\mathrm{H} 44$ & -0.244229 & 1.072262 & 0.132427 & 1 & 0.038 Uiso \\
\hline $\mathrm{C} 45$ & $-0.1739(5)$ & $0.9928(4)$ & $0.2350(4)$ & 1 & $0.0298(12)$ Uani \\
\hline H45 & -0.219222 & 1.019359 & 0.276590 & 1 & $0.036 \quad$ Uiso \\
\hline C46 & $-0.0926(5)$ & $0.9228(4)$ & $0.2603(3)$ & 1 & $0.0248(11)$ Uani \\
\hline H46 & -0.082041 & 0.902497 & 0.319267 & 1 & $0.030 \quad$ Uiso \\
\hline C47 & $0.1402(4)$ & $0.8487(4)$ & $0.3733(3)$ & 1 & 0.0218(10) Uani \\
\hline C48 & $0.1754(5)$ & $0.9414(4)$ & $0.3674(4)$ & 1 & 0.0294(12) Uani \\
\hline $\mathrm{H} 48$ & 0.194631 & 0.965010 & 0.313267 & 1 & 0.035 Uiso \\
\hline C49 & $0.1831(6)$ & $1.0006(5)$ & $0.4399(4)$ & 1 & 0.0379(14) Uani \\
\hline $\mathrm{H} 49$ & 0.207968 & 1.064237 & 0.435503 & 1 & 0.045 Uiso \\
\hline C50 & $0.1543(6)$ & $0.9660(5)$ & $0.5185(4)$ & 1 & $0.0428(16)$ Uani \\
\hline $\mathrm{H} 50$ & 0.157957 & 1.006455 & 0.567787 & 1 & $0.051 \quad$ Uiso \\
\hline C51 & $0.1205(6)$ & $0.8740(5)$ & $0.5258(4)$ & 1 & 0.0438(17) Uani \\
\hline H51 & 0.102168 & 0.850862 & 0.580285 & 1 & 0.053 Uiso \\
\hline C52 & $0.1129(5)$ & $0.8145(4)$ & $0.4539(4)$ & 1 & 0.0314(13) Uani \\
\hline H52 & 0.089309 & 0.750685 & 0.459178 & 1 & $0.038 \quad$ Uiso \\
\hline $\mathrm{Cl} 4$ & $0.1624(3)$ & $0.31712(16)$ & $0.16303(19)$ & 1 & $0.0831(7)$ Uani \\
\hline $\mathrm{Cl} 3$ & $0.0748(3)$ & $0.1532(2)$ & $0.2416(2)$ & 1 & 0.0999(10) Uani \\
\hline $\mathrm{Cl} 5$ & $0.4704(3)$ & $0.2695(2) \quad c$ & $0.19084(15)$ & 1 & 0.0903(9) Uani \\
\hline $\mathrm{Cl} 6$ & $0.6388(2)$ & $0.18056(17)$ & $0.32189(19)$ & 1 & $0.0791(7) \quad$ Uani \\
\hline C53 & $0.1559(10)$ & $0.1994(7)$ & $0.1564(7)$ & 1 & $0.087(3) \quad$ Uani \\
\hline $\mathrm{H} 53 \mathrm{~A}$ & 0.238616 & 0.174682 & 0.163040 & 1 & 0.104 \\
\hline H53B & 0.114410 & 0.179501 & 0.098503 & 1 & 0.104 \\
\hline C54 & $0.5343(14)$ & $0.1669(9)$ & $0.2337(8)$ & 1 & $0.125(6)$ \\
\hline $\mathrm{H} 54 \mathrm{~A}$ & 0.468814 & 0.125165 & 0.249547 & 1 & 0.150 \\
\hline H54B & 0.571893 & 0.134979 & 0.187311 & 1 & 0.150 \\
\hline
\end{tabular}

Table S12. Bond lengths $[\AA ̊]$ for $5 a$.

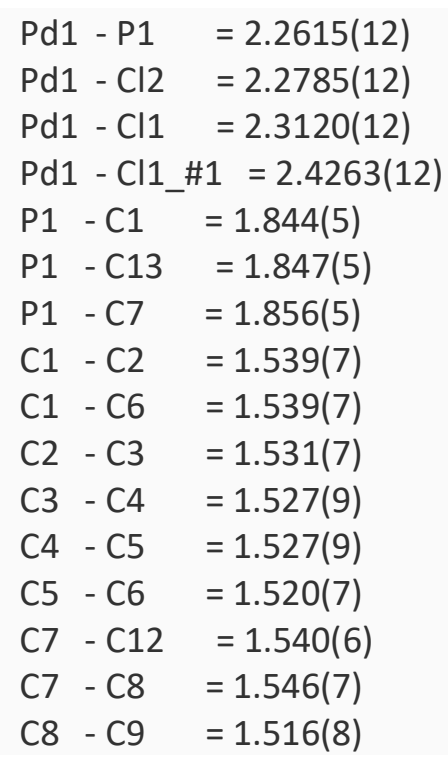




$$
\begin{aligned}
& \mathrm{C} 9-\mathrm{C} 10=1.532(8) \\
& \mathrm{C} 10-\mathrm{C} 11=1.523(8) \\
& \mathrm{C} 11-\mathrm{C} 12=1.525(7) \\
& \mathrm{C} 13-\mathrm{C} 18=1.404(6) \\
& \mathrm{C} 13-\mathrm{C} 14=1.412(7) \\
& \mathrm{C} 14-\mathrm{C} 15=1.379(7) \\
& \mathrm{C} 15-\mathrm{C} 16=1.384(8) \\
& \mathrm{C} 16-\mathrm{C} 17=1.373(7) \\
& \mathrm{C} 17-\mathrm{C} 18=1.404(7) \\
& \mathrm{C} 18-\mathrm{C} 19=1.504(7) \\
& \mathrm{C} 19-\mathrm{C} 24=1.411(7) \\
& \mathrm{C} 19-\mathrm{C} 20=1.414(7) \\
& \mathrm{C} 20-\mathrm{C} 21=1.397(8) \\
& \mathrm{C} 20-\mathrm{C} 25=1.499(7) \\
& \mathrm{C} 21-\mathrm{C} 22=1.379(8) \\
& \mathrm{C} 22-\mathrm{C} 23=1.378(8) \\
& \mathrm{C} 23-\mathrm{C} 24=1.398(7) \\
& \mathrm{C} 24-\mathrm{C} 39=1.501(7) \\
& \mathrm{C} 25-\mathrm{C} 26=1.349(7) \\
& \mathrm{C} 25-\mathrm{C} 33=1.485(7) \\
& \mathrm{C} 26-\mathrm{C} 27=1.499(8) \\
& \mathrm{C} 27-\mathrm{C} 28=1.387(8) \\
& \mathrm{C} 27-\mathrm{C} 32=1.397(8) \\
& \mathrm{C} 28-\mathrm{C} 29=1.389(9) \\
& \mathrm{C} 29-\mathrm{C} 30=1.388(11) \\
& \mathrm{C} 30-\mathrm{C} 31=1.379(10) \\
& \mathrm{C} 31-\mathrm{C} 32=1.393(8) \\
& \mathrm{C} 33-\mathrm{C} 34=1.395(8) \\
& \mathrm{C} 33-\mathrm{C} 38=1.398(8) \\
& \mathrm{C} 34-\mathrm{C} 35=1.388(9) \\
& \mathrm{C} 35-\mathrm{C} 36=1.391(11) \\
& \mathrm{C} 36-\mathrm{C} 37=1.368(12) \\
& \mathrm{C} 37-\mathrm{C} 38=1.388(9) \\
& \mathrm{C} 39-\mathrm{C} 40=1.342(7) \\
& \mathrm{C} 39-\mathrm{C} 47=1.489(7) \\
& \mathrm{C} 40-\mathrm{C} 41=1.481(7) \\
& \mathrm{C} 41-\mathrm{C} 46=1.389(7) \\
& \mathrm{C} 41-\mathrm{C} 42=1.399(7) \\
& \mathrm{C} 42-\mathrm{C} 43=1.378(8) \\
& \mathrm{C} 43-\mathrm{C} 44=1.382(8) \\
& \mathrm{C} 44-\mathrm{C} 45=1.384(8) \\
& \mathrm{C} 45-\mathrm{C} 46=1.390(7) \\
& \mathrm{C} 47-\mathrm{C} 48=1.381(8) \\
& \mathrm{C} 47-\mathrm{C} 52=1.406(7) \\
& \mathrm{C} 48-\mathrm{C} 49=1.393(8) \\
& \mathrm{C} 49-\mathrm{C} 50=1.385(9) \\
& \mathrm{C} 50-\mathrm{C} 51=1.369(10) \\
& \mathrm{C} 51-\mathrm{C} 52=1.388(9) \\
& \mathrm{Cl} 4-\mathrm{C} 53=1.685(10) \\
& \mathrm{Cl} 3-\mathrm{C} 53=1.809(13) \\
& \mathrm{Cl} 5-\mathrm{C} 54=1.740(12) \\
& \mathrm{Cl} 6-\mathrm{C} 54=1.683(12)
\end{aligned}
$$


Table S13. Angles $\left[^{\circ}\right]$ for $5 a$.

\begin{tabular}{|c|c|c|c|}
\hline P1 & - Pd1 & $-\mathrm{Cl} 2$ & $=85.85(4)$ \\
\hline P1 & - Pd1 & $-\mathrm{Cl} 1$ & $=99.74(4)$ \\
\hline $\mathrm{Cl} 2$ & - Pd1 & $-\mathrm{Cl} 1=$ & $=173.81(4)$ \\
\hline P1 & - Pd1 & - Cl1_\#1 & $1=175.44(4)$ \\
\hline $\mathrm{Cl} 2$ & - Pd1 & - Cl1_\#1 & $1=89.60(4)$ \\
\hline $\mathrm{Cl} 1$ & - Pd1 & - Cl1_\#1 & $1=84.81(4)$ \\
\hline Pd1 & $-\mathrm{Cl} 1$ & - Pd1_\#- & $\# 1=95.19(4)$ \\
\hline C1 & $-P 1$ & $-\mathrm{C} 13=$ & $=104.2(2)$ \\
\hline $\mathrm{C} 1$ & $-\mathrm{P} 1$ & $-\mathrm{C7}$ & $=107.1(2)$ \\
\hline C13 & $-\mathrm{P} 1$ & $-\mathrm{C7}$ & $=114.8(2)$ \\
\hline C1 & $-P 1$ & - Pd1 & $=118.79(16)$ \\
\hline C13 & - P1 & - Pd1 & $=105.55(15)$ \\
\hline $\mathrm{C7}$ & $-P 1$ & $-\mathrm{Pd} 1$ & $=106.85(15)$ \\
\hline C2 & $-\mathrm{C} 1$ & $-\mathrm{C} 6$ & $=109.8(4)$ \\
\hline C2 & $-\mathrm{C} 1$ & $-P 1$ & $=114.9(3)$ \\
\hline C6 & $-\mathrm{C} 1$ & $-P 1$ & $=113.7(3)$ \\
\hline C3 & $-\mathrm{C} 2$ & $-\mathrm{C} 1$ & $=110.7(4)$ \\
\hline $\mathrm{C} 4$ & $-\mathrm{C} 3$ & $-\mathrm{C} 2$ & $=111.6(5)$ \\
\hline C3 & $-\mathrm{C} 4$ & $-\mathrm{C5}$ & $=110.7(5)$ \\
\hline C6 & $-\mathrm{C5}$ & $-\mathrm{C} 4$ & $=111.4(5)$ \\
\hline C5 & $-\mathrm{C} 6$ & $-\mathrm{C} 1$ & $=110.2(4)$ \\
\hline C12 & $-\mathrm{C7}$ & $-\mathrm{C} 8$ & $=110.0(4)$ \\
\hline C12 & $-\mathrm{C7}$ & $-\mathrm{P} 1$ & $=117.1(3)$ \\
\hline $\mathrm{C} 8$ & $-\mathrm{C7}$ & $-P 1$ & $=110.3(3)$ \\
\hline C9 & $-\mathrm{C} 8$ & $-\mathrm{C7}$ & $=112.3(4)$ \\
\hline $\mathrm{C} 8$ & $-\mathrm{CP}$ & $-\mathrm{C} 10$ & $=111.7(5)$ \\
\hline C11 & - C10 & - C9 & $=110.3(4)$ \\
\hline C10 & $-\mathrm{C} 11$ & $-\mathrm{C} 12$ & $=111.9(4)$ \\
\hline C11 & $-\mathrm{C} 12$ & $-\mathrm{C7}$ & $=111.0(4)$ \\
\hline C18 & $-\mathrm{C} 13$ & - C14 & $=117.8(4)$ \\
\hline C18 & $-\mathrm{C} 13$ & $-P 1$ & $=130.5(4)$ \\
\hline C14 & $-\mathrm{C} 13$ & $-P 1$ & $=111.7(3)$ \\
\hline C15 & $-\mathrm{C} 14$ & $-\mathrm{C} 13$ & $=122.2(5)$ \\
\hline C14 & $-\mathrm{C} 15$ & - C16 & $=119.8(5)$ \\
\hline C17 & $-\mathrm{C} 16$ & - C15 & $=118.8(5)$ \\
\hline C16 & $-\mathrm{C} 17$ & - C18 & $=122.9(5)$ \\
\hline C17 & $-\mathrm{C} 18$ & $-\mathrm{C} 13$ & $=118.4(4)$ \\
\hline C17 & $-\mathrm{C} 18$ & - C19 & $=115.6(4)$ \\
\hline $\mathrm{C} 13$ & $-\mathrm{C} 18$ & - C19 & $=125.9(4)$ \\
\hline C24 & - C19 & - C20 & $=119.7(5)$ \\
\hline C24 & - C19 & $-\mathrm{C} 18$ & $=119.8(4)$ \\
\hline C20 & - C19 & - C18 & $=120.4(4)$ \\
\hline C21 & $-\mathrm{C} 20$ & - C19 & $=118.5(5)$ \\
\hline $\mathrm{C} 21$ & $-\mathrm{C} 20$ & $-\mathrm{C} 25$ & $=116.8(5)$ \\
\hline C19 & $-\mathrm{C} 20$ & $-\mathrm{C} 25$ & $=124.7(5)$ \\
\hline $\mathrm{C} 22$ & $-\mathrm{C} 21$ & $-\mathrm{C} 20$ & $=121.7(5)$ \\
\hline C23 & $-\mathrm{C} 22$ & $-C 21$ & $=119.8(5)$ \\
\hline $\mathrm{C} 22$ & $-\mathrm{C} 23$ & $-C 24$ & $=120.9(5)$ \\
\hline $\mathrm{C} 23$ & $-\mathrm{C} 24$ & - C19 & $=119.3(5)$ \\
\hline $\mathrm{C} 23$ & $-C 24$ & - С39 & $=118.1(5)$ \\
\hline
\end{tabular}




\begin{tabular}{|c|c|c|c|}
\hline C19 & $-\mathrm{C} 24$ & - C39 & $=122.6(5)$ \\
\hline C26 & $-C 25$ & - C33 & $=121.7(5)$ \\
\hline C26 & $-\mathrm{C} 25$ & $-\mathrm{C} 20$ & $=118.9(5)$ \\
\hline C33 & $-\mathrm{C} 25$ & $-\mathrm{C} 20$ & $=119.1(5)$ \\
\hline C25 & $-\mathrm{C} 26$ & $-C 27$ & $=129.3(5)$ \\
\hline C28 & $-\mathrm{C} 27$ & - C32 & $=118.1(5)$ \\
\hline C28 & $-\mathrm{C} 27$ & $-\mathrm{C} 26$ & $=118.5(5)$ \\
\hline C32 & $-C 27$ & $-C 26$ & $=123.3(5)$ \\
\hline C27 & $-C 28$ & $-C 29$ & $=121.9(6)$ \\
\hline C30 & $-C 29$ & $-\mathrm{C} 28$ & $=119.0(6)$ \\
\hline C31 & $-\mathrm{C30}$ & $-\mathrm{C} 29$ & $=120.3(6)$ \\
\hline C30 & - C31 & - C32 & $=120.3(7)$ \\
\hline C31 & $-\mathrm{C} 32$ & $-\mathrm{C} 27$ & $=120.4(6)$ \\
\hline C34 & $-\mathrm{C} 33$ & $-\mathrm{C} 38$ & $=118.0(5)$ \\
\hline C34 & - C33 & $-\mathrm{C} 25$ & $=122.0(5)$ \\
\hline C38 & - C33 & $-\mathrm{C} 25$ & $=120.0(5)$ \\
\hline C35 & - C34 & $-\mathrm{C} 33$ & $=120.6(6)$ \\
\hline C34 & - C35 & - C36 & $=120.2(7)$ \\
\hline C37 & - C36 & $-\mathrm{C} 35$ & $=120.0(6)$ \\
\hline C36 & - C37 & - С38 & $=120.0(7)$ \\
\hline C37 & $-\mathrm{C} 38$ & $-\mathrm{C33}$ & $=121.3(6)$ \\
\hline C40 & - С39 & $-\mathrm{C} 47$ & $=123.0(5)$ \\
\hline C40 & - C39 & $-\mathrm{C} 24$ & $=119.3(5)$ \\
\hline C47 & - C39 & $-\mathrm{C} 24$ & $=117.5(4)$ \\
\hline C39 & - C40 & - C41 & $=129.7(5)$ \\
\hline C46 & - C41 & $-\mathrm{C} 42$ & $=118.2(5)$ \\
\hline C46 & - C41 & $-\mathrm{C} 40$ & $=123.2(4)$ \\
\hline C42 & - C41 & $-\mathrm{C} 40$ & $=118.4(5)$ \\
\hline C43 & $-\mathrm{C} 42$ & - C41 & $=120.7(5)$ \\
\hline C42 & $-\mathrm{C} 43$ & $-\mathrm{C} 44$ & $=120.7(5)$ \\
\hline C43 & - C44 & $-\mathrm{C} 45$ & $=119.2(5)$ \\
\hline C44 & - C45 & $-\mathrm{C} 46$ & $=120.4(5)$ \\
\hline C41 & - C46 & $-\mathrm{C} 45$ & $=120.7(5)$ \\
\hline C48 & $-\mathrm{C} 47$ & $-\mathrm{C} 52$ & $=119.1(5)$ \\
\hline C48 & - C47 & - C39 & $=121.2(5)$ \\
\hline C52 & $-\mathrm{C} 47$ & - С39 & $=119.7(5)$ \\
\hline C47 & $-\mathrm{C} 48$ & $-C 49$ & $=120.7(6)$ \\
\hline $\mathrm{C} 50$ & - C49 & $-\mathrm{C} 48$ & $=119.4(6)$ \\
\hline C51 & $-\mathrm{C} 50$ & $-C 49$ & $=120.7(6)$ \\
\hline C50 & - C51 & - C52 & $=120.3(6)$ \\
\hline C51 & $-\mathrm{C} 52$ & $-\mathrm{C} 47$ & $=119.8(6)$ \\
\hline $\mathrm{Cl} 4$ & - C53 & $-\mathrm{Cl} 3$ & $=109.5(6)$ \\
\hline $\mathrm{Cl} 6$ & - C54 & $-\mathrm{Cl} 5$ & $=115.6(7)$ \\
\hline
\end{tabular}

Table S11. Torsion angles $\left[^{\circ}\right]$ for $5 a$.

$\begin{array}{llllc}\mathrm{C} 13 & -\mathrm{P} 1 & -\mathrm{C} 1 & -\mathrm{C} 2 & =179.9(3) \\ \mathrm{C} 7 & -\mathrm{P} 1 & -\mathrm{C} 1 & -\mathrm{C} 2 & =57.8(4) \\ \mathrm{Pd} 1 & -\mathrm{P} 1 & -\mathrm{C} 1 & -\mathrm{C} 2 & =-63.1(4) \\ \mathrm{C} 13 & -\mathrm{P} 1 & -\mathrm{C} 1 & -\mathrm{C} 6 & =-52.5(4) \\ \mathrm{C} 7 & -\mathrm{P} 1 & -\mathrm{C} 1 & -\mathrm{C} 6 & =-174.5(3) \\ \mathrm{Pd} 1 & -\mathrm{P} 1 & -\mathrm{C} 1 & -\mathrm{C} 6 & =64.5(4)\end{array}$




\begin{tabular}{|c|c|c|c|c|}
\hline C6 & $-\mathrm{C} 1$ & $-\mathrm{C} 2$ & $-\mathrm{C} 3$ & $=57.1(5)$ \\
\hline P1 & $-\mathrm{C} 1$ & $-\mathrm{C} 2$ & $-\mathrm{C} 3$ & $=-173.3(4)$ \\
\hline $\mathrm{C} 1$ & $-\mathrm{C} 2$ & $-\mathrm{C} 3$ & $-\mathrm{C} 4$ & $=-55.8(6)$ \\
\hline $\mathrm{C} 2$ & $-\mathrm{C3}$ & $-\mathrm{C} 4$ & $-\mathrm{C} 5$ & $=54.8(7)$ \\
\hline C3 & $-\mathrm{C} 4$ & $-\mathrm{C5}$ & $-\mathrm{C} 6$ & $=-56.2(6)$ \\
\hline C4 & $-\mathrm{C5}$ & $-\mathrm{C} 6$ & $-\mathrm{C} 1$ & $=58.1(6)$ \\
\hline$C 2$ & $-\mathrm{C} 1$ & $-\mathrm{C} 6$ & $-\mathrm{C} 5$ & $=-58.2(5)$ \\
\hline P1 & $-\mathrm{C} 1$ & $-\mathrm{C} 6$ & $-\mathrm{C} 5$ & $=171.6(4)$ \\
\hline $\mathrm{C} 1$ & $-P 1$ & $-\mathrm{C7}$ & $-\mathrm{C} 12$ & $=29.6(4)$ \\
\hline $\mathrm{C} 13$ & - P1 & $-\mathrm{C7}$ & - C12 & $=-85.5(4)$ \\
\hline Pd1 & $-\mathrm{P} 1$ & $-\mathrm{C7}$ & - C12 & $=157.9(3)$ \\
\hline C1 & $-P 1$ & $-\mathrm{C7}$ & $-\mathrm{C} 8$ & $=-97.2(4)$ \\
\hline C13 & $-\mathrm{P} 1$ & $-\mathrm{C7}$ & $-\mathrm{C} 8$ & $=147.7(3)$ \\
\hline Pd1 & $-P 1$ & $-C 7$ & $-\mathrm{C} 8$ & $=31.1(4)$ \\
\hline C12 & $-C 7$ & $-\mathrm{C} 8$ & $-\mathrm{CP}$ & $=54.2(6)$ \\
\hline P1 & $-\mathrm{C7}$ & $-\mathrm{C} 8$ & - C9 & $=-175.2(4)$ \\
\hline $\mathrm{C7}$ & $-\mathrm{C} 8$ & $-\mathrm{C9}$ & $-\mathrm{C} 10$ & $=-54.9(6)$ \\
\hline C8 & $-\mathrm{CP}$ & $-\mathrm{C} 10$ & - C11 & $=55.3(7)$ \\
\hline C9 & - C10 & $-\mathrm{C} 11$ & - C12 & $=-56.7(6)$ \\
\hline C10 & - C11 & - C12 & $-C 7$ & $=57.2(6)$ \\
\hline $\mathrm{C} 8$ & $-C 7$ & - C12 & - C11 & $=-54.7(5)$ \\
\hline P1 & $-C 7$ & $-\mathrm{C} 12$ & - C11 & $=178.4(3)$ \\
\hline $\mathrm{C} 1$ & $-P 1$ & $-\mathrm{C} 13$ & $-\mathrm{C} 18$ & $=-91.5(5)$ \\
\hline C7 & $-\mathrm{P} 1$ & - C13 & - C18 & $=25.2(5)$ \\
\hline Pd1 & $-\mathrm{P} 1$ & - C13 & - C18 & $=142.6(4)$ \\
\hline $\mathrm{C} 1$ & $-P 1$ & $-\mathrm{C} 13$ & $-\mathrm{C} 14$ & $=88.9(4)$ \\
\hline C7 & $-P 1$ & $-\mathrm{C} 13$ & - C14 & $=-154.4(3)$ \\
\hline Pd1 & $-P 1$ & - C13 & - C14 & $=-37.0(4)$ \\
\hline C18 & - C13 & - C14 & - C15 & $=0.5(7)$ \\
\hline P1 & $-\mathrm{C} 13$ & - C14 & - C15 & $=-179.9(4)$ \\
\hline C13 & - C14 & - C15 & - C16 & $=-2.5(8)$ \\
\hline C14 & $-\mathrm{C} 15$ & $-\mathrm{C} 16$ & $-\mathrm{C} 17$ & $=1.7(8)$ \\
\hline C15 & $-\mathrm{C} 16$ & $-\mathrm{C} 17$ & $-\mathrm{C} 18$ & $=1.1(8)$ \\
\hline C16 & $-\mathrm{C} 17$ & $-\mathrm{C} 18$ & $-\mathrm{C} 13$ & $=-3.1(7)$ \\
\hline C16 & $-\mathrm{C} 17$ & - C18 & $-\mathrm{C} 19$ & $=174.9(5)$ \\
\hline C14 & - C13 & - C18 & - C17 & $=2.2(6)$ \\
\hline P1 & $-\mathrm{C} 13$ & $-\mathrm{C} 18$ & - C17 & $=-177.3(4)$ \\
\hline C14 & $-\mathrm{C} 13$ & - C18 & - C19 & $=-175.5(4$ \\
\hline P1 & $-\mathrm{C} 13$ & $-\mathrm{C} 18$ & - C19 & $=4.9(7)$ \\
\hline C17 & - C18 & - C19 & $-\mathrm{C} 24$ & $=88.0(5)$ \\
\hline C13 & $-\mathrm{C} 18$ & $-\mathrm{C} 19$ & $-\mathrm{C} 24$ & $=-94.2(6)$ \\
\hline C17 & $-\mathrm{C} 18$ & - C19 & $-\mathrm{C} 20$ & $=-88.6(5)$ \\
\hline C13 & $-\mathrm{C} 18$ & $-\mathrm{C} 19$ & $-\mathrm{C} 20$ & $=89.2(6)$ \\
\hline C24 & - C19 & $-\mathrm{C} 20$ & $-C 21$ & $=2.2(7)$ \\
\hline $\mathrm{C} 18$ & - C19 & $-\mathrm{C} 20$ & $-C 21$ & $=178.8(5)$ \\
\hline C24 & - C19 & $-\mathrm{C} 20$ & $-\mathrm{C} 25$ & $=-176.8(5$ \\
\hline C18 & $-\mathrm{C} 19$ & $-\mathrm{C} 20$ & $-\mathrm{C} 25$ & $=-0.2(7)$ \\
\hline C19 & $-\mathrm{C} 20$ & $-\mathrm{C} 21$ & $-\mathrm{C} 22$ & $=-0.1(8)$ \\
\hline C25 & $-\mathrm{C} 20$ & $-C 21$ & $-\mathrm{C} 22$ & $=178.9(5)$ \\
\hline C20 & $-C 21$ & $-\mathrm{C} 22$ & $-\mathrm{C} 23$ & $=-1.0(9)$ \\
\hline C21 & - C22 & $-\mathrm{C} 23$ & $-\mathrm{C} 24$ & $=0.1(9)$ \\
\hline C22 & $-\mathrm{C} 23$ & $-\mathrm{C} 24$ & - C19 & $=2.0(8)$ \\
\hline
\end{tabular}




\begin{tabular}{|c|c|c|c|c|}
\hline $\mathrm{C} 22$ & $-\mathrm{C} 23$ & - C24 & - C39 & $=-178.2(5)$ \\
\hline C20 & - C19 & $-C 24$ & $-C 23$ & $=-3.2(7)$ \\
\hline C18 & $-\mathrm{C} 19$ & $-C 24$ & $-\mathrm{C} 23$ & $=-179.8(4)$ \\
\hline C20 & $-\mathrm{C} 19$ & $-\mathrm{C} 24$ & - C39 & $=177.1(4)$ \\
\hline C18 & $-\mathrm{C} 19$ & $-\mathrm{C} 24$ & - C39 & $=0.5(7)$ \\
\hline C21 & $-\mathrm{C} 20$ & $-\mathrm{C} 25$ & $-\mathrm{C} 26$ & $=44.7(7)$ \\
\hline C19 & $-\mathrm{C} 20$ & $-\mathrm{C} 25$ & $-\mathrm{C} 26$ & $=-136.3(5)$ \\
\hline C21 & $-\mathrm{C} 20$ & $-\mathrm{C} 25$ & $-\mathrm{C} 33$ & $=-129.6(5)$ \\
\hline C19 & $-\mathrm{C} 20$ & $-\mathrm{C} 25$ & $-\mathrm{C} 33$ & $=49.4(7)$ \\
\hline C33 & $-C 25$ & $-C 26$ & $-C 27$ & $=7.6(8)$ \\
\hline C2O & $-\mathrm{C} 25$ & $-\mathrm{C} 26$ & $-\mathrm{C} 27$ & $=-166.5(5)$ \\
\hline $\mathrm{C} 25$ & $-\mathrm{C} 26$ & $-\mathrm{C} 27$ & $-\mathrm{C} 28$ & $=-157.0(6)$ \\
\hline C25 & $-C 26$ & $-C 27$ & - C32 & $=27.7(9)$ \\
\hline C32 & $-\mathrm{C} 27$ & $-\mathrm{C} 28$ & $-C 29$ & $=-2.5(9)$ \\
\hline C26 & $-\mathrm{C} 27$ & $-\mathrm{C} 28$ & $-C 29$ & $=-178.0(6)$ \\
\hline $\mathrm{C} 27$ & $-\mathrm{C} 28$ & $-\mathrm{C} 29$ & $-\mathrm{C} 30$ & $=3.0(11)$ \\
\hline C28 & $-\mathrm{C} 29$ & - C30 & - C31 & $=-2.4(11)$ \\
\hline C29 & $-\mathrm{C} 30$ & $-\mathrm{C} 31$ & $-\mathrm{C} 32$ & $=1.3(10)$ \\
\hline C30 & $-\mathrm{C} 31$ & $-\mathrm{C} 32$ & $-\mathrm{C} 27$ & $=-0.8(10)$ \\
\hline C28 & $-\mathrm{C} 27$ & $-\mathrm{C} 32$ & $-\mathrm{C} 31$ & $=1.3(9)$ \\
\hline C26 & $-\mathrm{C} 27$ & $-\mathrm{C} 32$ & $-\mathrm{C} 31$ & $=176.6(5)$ \\
\hline C26 & $-\mathrm{C} 25$ & - C33 & - C34 & $=55.2(7)$ \\
\hline C20 & $-\mathrm{C} 25$ & $-\mathrm{C} 33$ & $-\mathrm{C} 34$ & $=-130.6(5)$ \\
\hline C26 & $-\mathrm{C} 25$ & - C33 & $-\mathrm{C} 38$ & $=-123.1(6)$ \\
\hline C20 & $-\mathrm{C} 25$ & $-\mathrm{C} 33$ & $-\mathrm{C} 38$ & $=51.0(7)$ \\
\hline C38 & $-\mathrm{C} 33$ & $-\mathrm{C} 34$ & $-\mathrm{C} 35$ & $=0.8(8)$ \\
\hline $\mathrm{C} 25$ & $-\mathrm{C} 33$ & - C34 & $-\mathrm{C} 35$ & $=-177.6(5)$ \\
\hline C33 & $-\mathrm{C} 34$ & $-\mathrm{C} 35$ & $-\mathrm{C} 36$ & $=-0.5(9)$ \\
\hline C34 & $-\mathrm{C} 35$ & $-\mathrm{C} 36$ & $-\mathrm{C} 37$ & $=-0.5(10)$ \\
\hline C35 & $-\mathrm{C} 36$ & - C37 & $-\mathrm{C} 38$ & $=1.1(10)$ \\
\hline C36 & $-\mathrm{C} 37$ & $-\mathrm{C} 38$ & $-\mathrm{C} 33$ & $=-0.9(9)$ \\
\hline C34 & $-\mathrm{C} 33$ & $-\mathrm{C} 38$ & $-\mathrm{C} 37$ & $=-0.1(8)$ \\
\hline $\mathrm{C} 25$ & $-\mathrm{C} 33$ & $-\mathrm{C} 38$ & $-\mathrm{C} 37$ & $=178.3(5)$ \\
\hline $\mathrm{C} 23$ & $-\mathrm{C} 24$ & - C39 & $-\mathrm{C} 40$ & $=-58.5(6)$ \\
\hline C19 & $-\mathrm{C} 24$ & - С39 & $-\mathrm{C} 40$ & $=121.3(5)$ \\
\hline $\mathrm{C} 23$ & $-\mathrm{C} 24$ & - С39 & $-\mathrm{C} 47$ & $=115.8(5)$ \\
\hline C19 & $-\mathrm{C} 24$ & - C39 & $-\mathrm{C} 47$ & $=-64.4(6)$ \\
\hline C47 & - C39 & $-\mathrm{C} 40$ & $-\mathrm{C} 41$ & $=-1.1(8)$ \\
\hline C24 & - C39 & $-\mathrm{C} 40$ & $-\mathrm{C} 41$ & $=172.9(5)$ \\
\hline C39 & $-\mathrm{C} 40$ & - C41 & $-\mathrm{C} 46$ & $=-37.1(8)$ \\
\hline C39 & $-\mathrm{C} 40$ & - C41 & $-\mathrm{C} 42$ & $=147.7(5)$ \\
\hline C46 & $-\mathrm{C} 41$ & $-\mathrm{C} 42$ & $-\mathrm{C} 43$ & $=2.2(8)$ \\
\hline $\mathrm{C} 40$ & $-\mathrm{C} 41$ & $-\mathrm{C} 42$ & $-\mathrm{C} 43$ & $=177.7(5)$ \\
\hline C41 & $-\mathrm{C} 42$ & $-\mathrm{C} 43$ & $-\mathrm{C} 44$ & $=-2.5(9)$ \\
\hline C42 & $-\mathrm{C} 43$ & - C44 & $-\mathrm{C} 45$ & $=1.1(9)$ \\
\hline $\mathrm{C} 43$ & $-\mathrm{C} 44$ & $-\mathrm{C} 45$ & $-\mathrm{C} 46$ & $=0.6(9)$ \\
\hline C42 & $-\mathrm{C} 41$ & - C46 & $-\mathrm{C} 45$ & $=-0.5(8)$ \\
\hline $\mathrm{C} 40$ & $-\mathrm{C} 41$ & - C46 & $-\mathrm{C} 45$ & $=-175.8(5)$ \\
\hline C44 & $-\mathrm{C} 45$ & $-\mathrm{C} 46$ & $-\mathrm{C} 41$ & $=-0.8(9)$ \\
\hline $\mathrm{C} 40$ & - C39 & $-\mathrm{C} 47$ & $-\mathrm{C} 48$ & $=-57.4(7)$ \\
\hline C24 & - C39 & $-\mathrm{C} 47$ & $-\mathrm{C} 48$ & $=128.5(5)$ \\
\hline C40 & - C39 & - C47 & $-\mathrm{C} 52$ & $=121.1(6)$ \\
\hline
\end{tabular}




$\begin{array}{lllll}\text { C24 } & - \text { C39 } & - \text { C47 } & - \text { C52 } & =-52.9(6) \\ \text { C52 } & - \text { C47 } & - \text { C48 } & - \text { C49 } & =-0.5(8) \\ \text { C39 } & - \text { C47 } & - \text { C48 } & - \text { C49 } & =178.1(5) \\ \text { C47 } & - \text { C48 } & - \text { C49 } & - \text { C50 } & =-0.4(9) \\ \text { C48 } & - \text { C49 } & - \text { C50 } & - \text { C51 } & =1.2(9) \\ \text { C49 } & - \text { C50 } & - \text { C51 } & - \text { C52 } & =-1.0(10) \\ \text { C50 } & - \text { C51 } & - \text { C52 } & - \text { C47 } & =0.1(9) \\ \text { C48 } & - \text { C47 } & - \text { C52 } & - \text { C51 } & =0.6(8) \\ \text { C39 } & - \text { C47 } & - \text { C52 } & - \text { C51 } & =-178.0(5)\end{array}$

\section{NMR Charts}




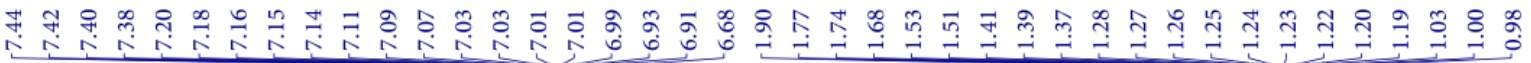<smiles>O=C(O)c1ccccc1-c1ccccc1C(=Cc1ccccc1)c1ccccc1</smiles>
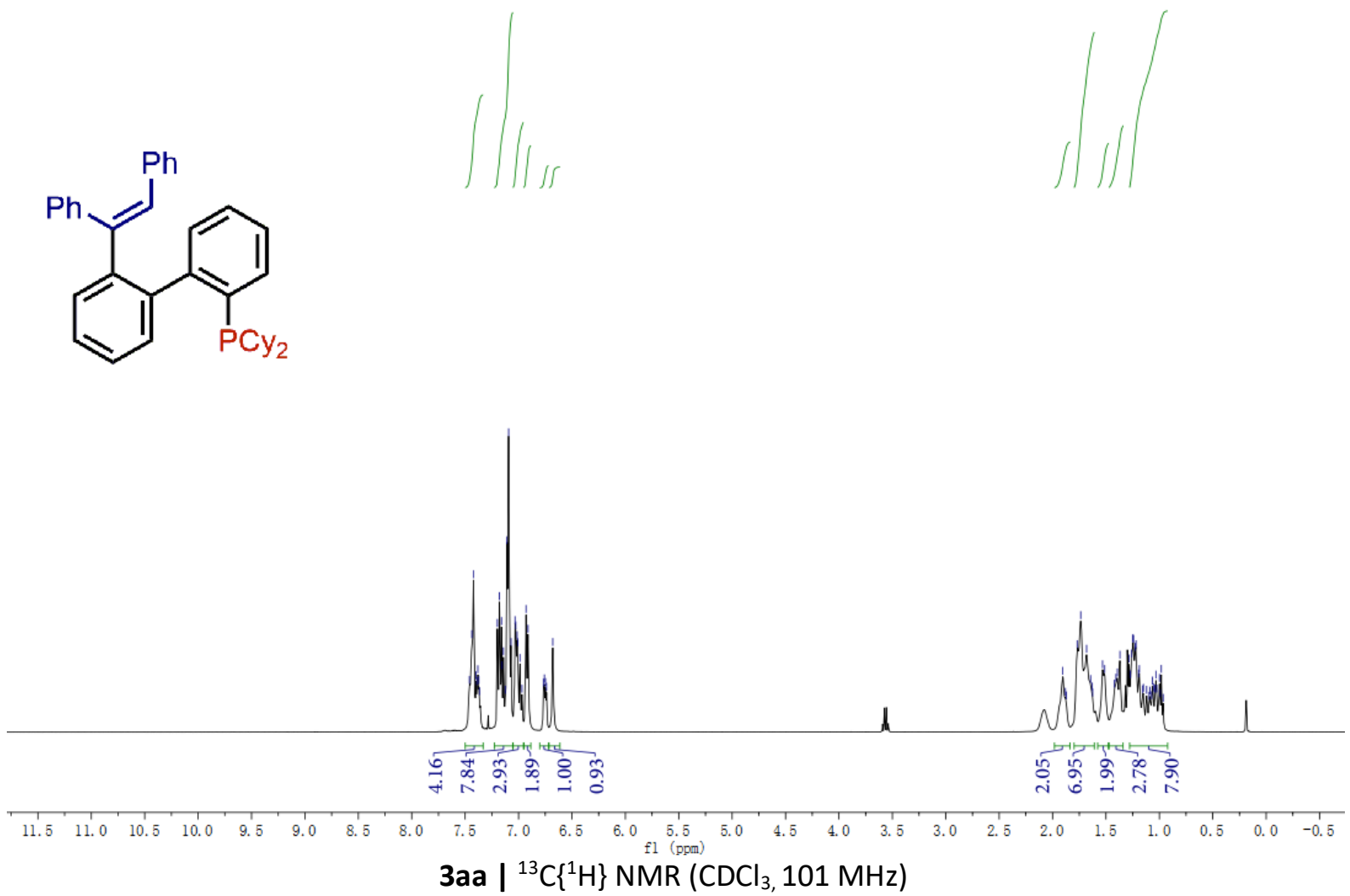

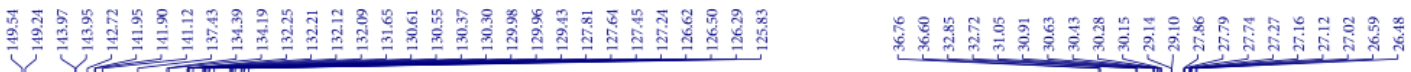<smiles>O=C(O)c1ccccc1-c1ccccc1C(=Cc1ccccc1)c1ccccc1</smiles>
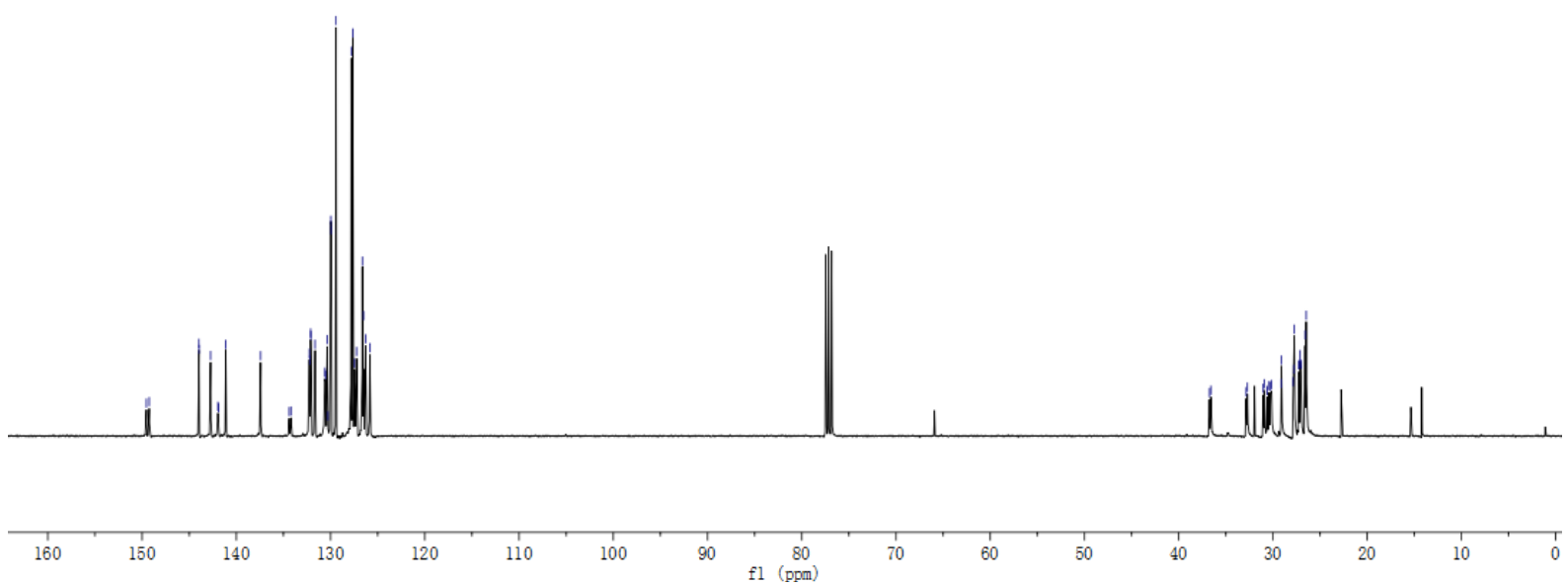
3aa | ${ }^{31} \mathrm{P}\left\{{ }^{1} \mathrm{H}\right\} \mathrm{NMR}\left(\mathrm{CDCl}_{3}, 162 \mathrm{MHz}\right)$

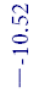<smiles>C(=C(c1ccccc1)c1ccccc1-c1ccccc1)c1ccccc1</smiles>

$\begin{array}{lllllllllllllllllllllllllllllllllll}140 & 130 & 120 & 110 & 100 & 90 & 80 & 70 & 60 & 50 & 40 & 30 & 20 & 10 & 0 & -10 & -20 & -30 & -40 & -50 & -60 & -70 & -80 & -90 & -100 & -110 & -120 & -130 & -140\end{array}$ 

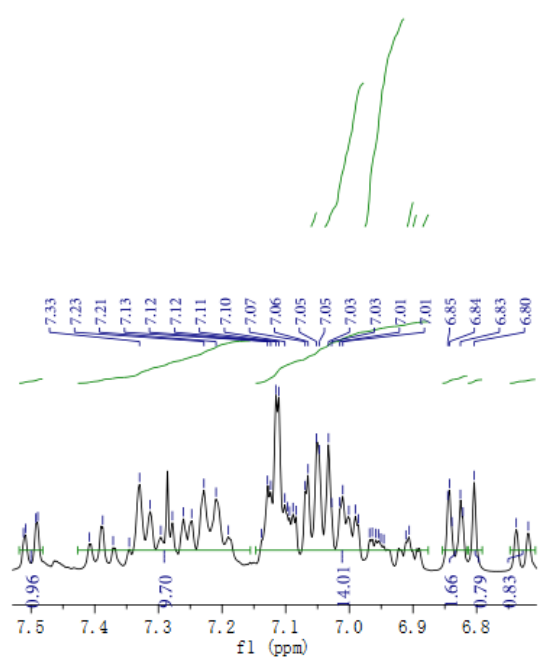<smiles>Pc1ccccc1-c1ccccc1-c1ccccc1-c1ccccc1</smiles>

\section{Win wh W}

(1)

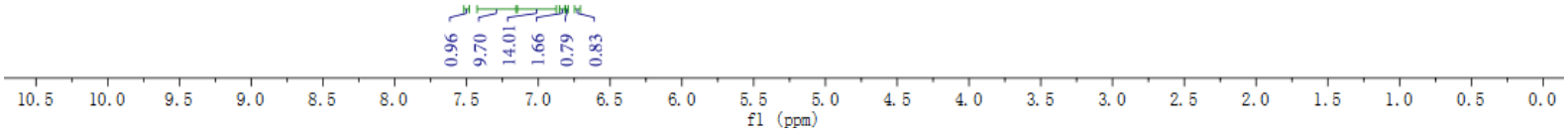

3ba $\mid{ }^{13} \mathrm{C}\left\{{ }^{1} \mathrm{H}\right\}$ NMR $\left(\mathrm{CDCl}_{3}, 101 \mathrm{MHz}\right)$

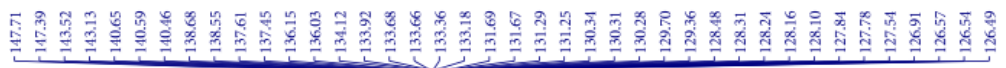

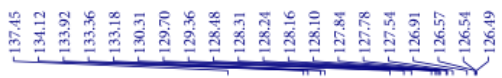
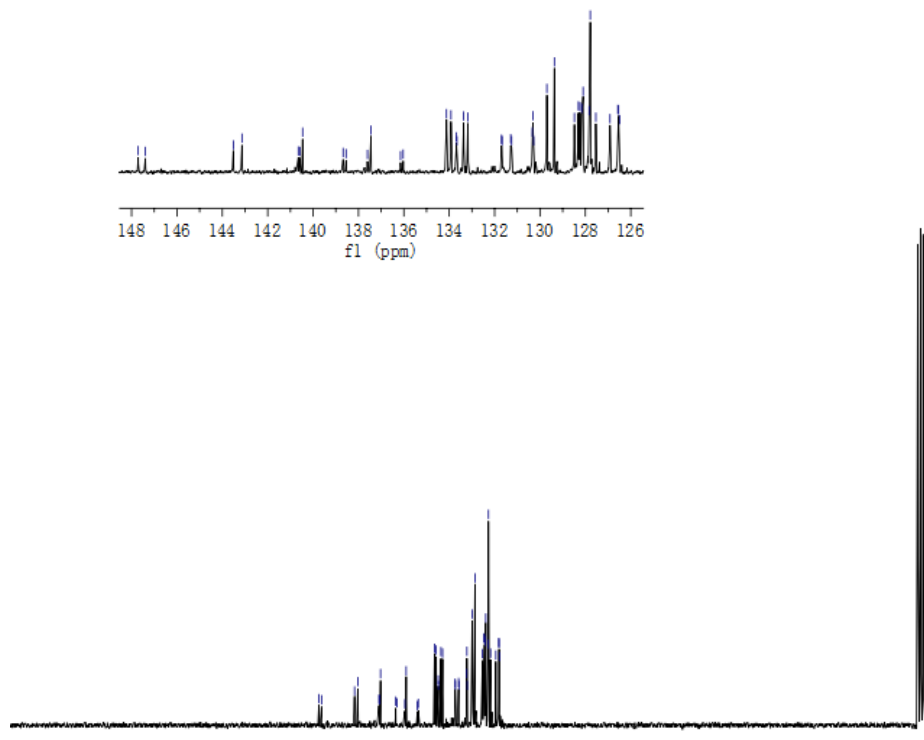<smiles>Pc1ccccc1-c1ccccc1-c1ccccc1-c1ccccc1</smiles>

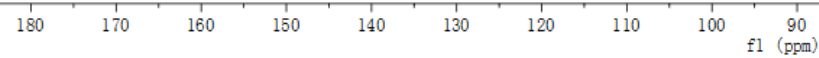


3ba | ${ }^{31} \mathrm{P}\left\{{ }^{1} \mathrm{H}\right\} \mathrm{NMR}\left(\mathrm{CDCl}_{3}, 162 \mathrm{MHz}\right)$<smiles>c1ccc(-c2ccccc2-c2ccccc2-c2ccccc2)cc1</smiles>

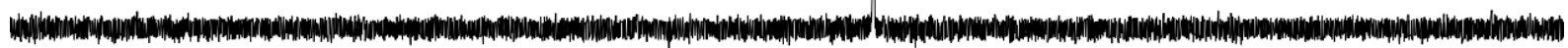

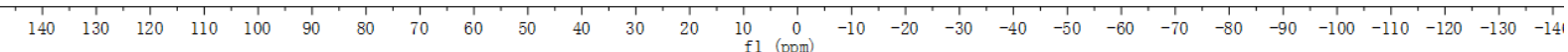


<smiles>O=[PH](=O)(O)c1ccccc1-c1ccccc1C(=Cc1ccccc1)c1ccccc1</smiles>
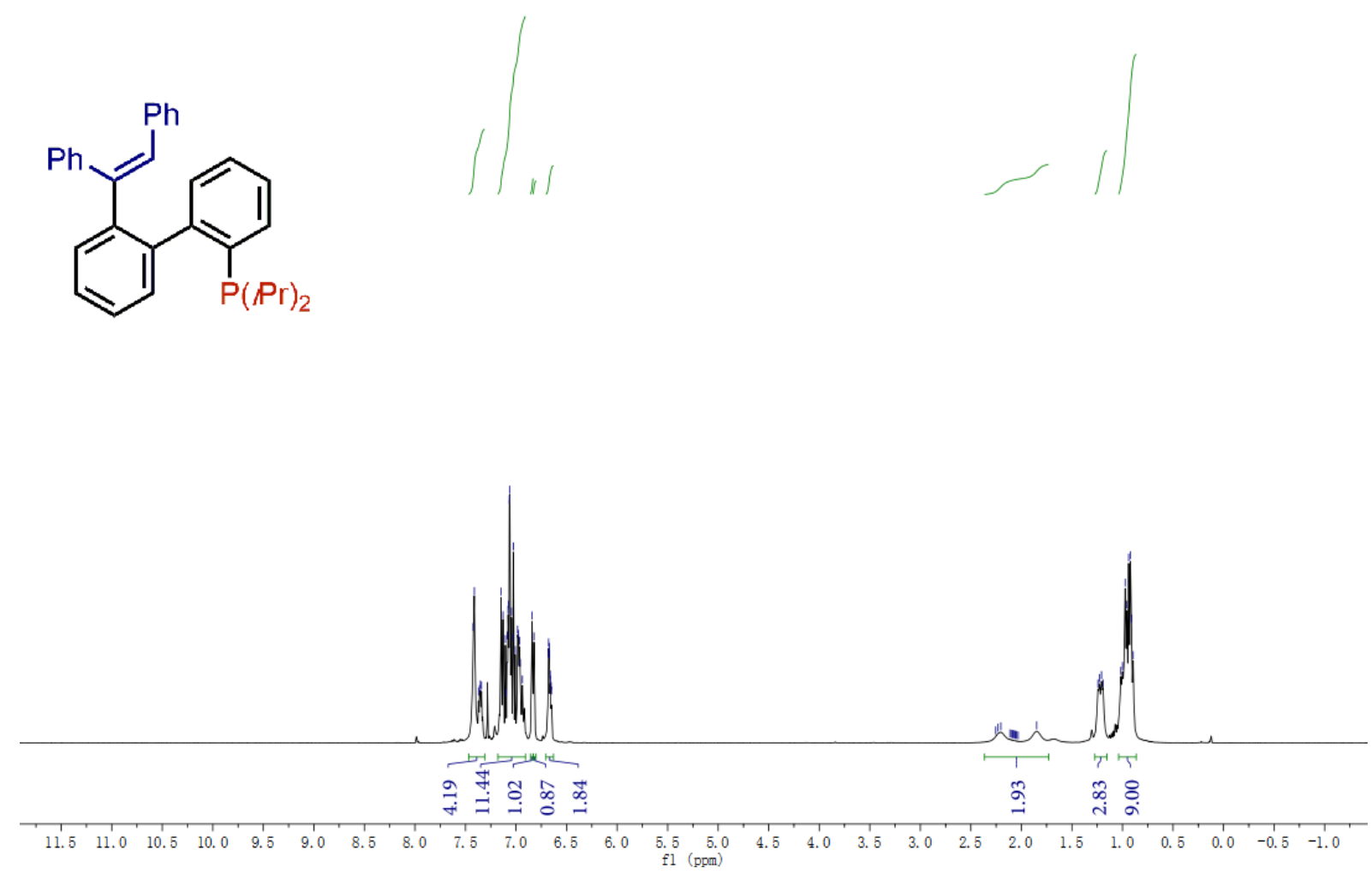

3ca $\mid{ }^{13} \mathrm{C}\left\{{ }^{1} \mathrm{H}\right\}$ NMR $\left(\mathrm{CDCl}_{3}, 101 \mathrm{MHz}\right)$

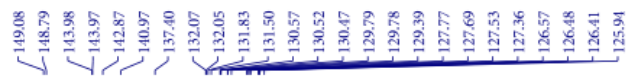<smiles>O=P(O)(O)c1ccccc1-c1ccccc1C(=Cc1ccccc1)c1ccccc1</smiles>

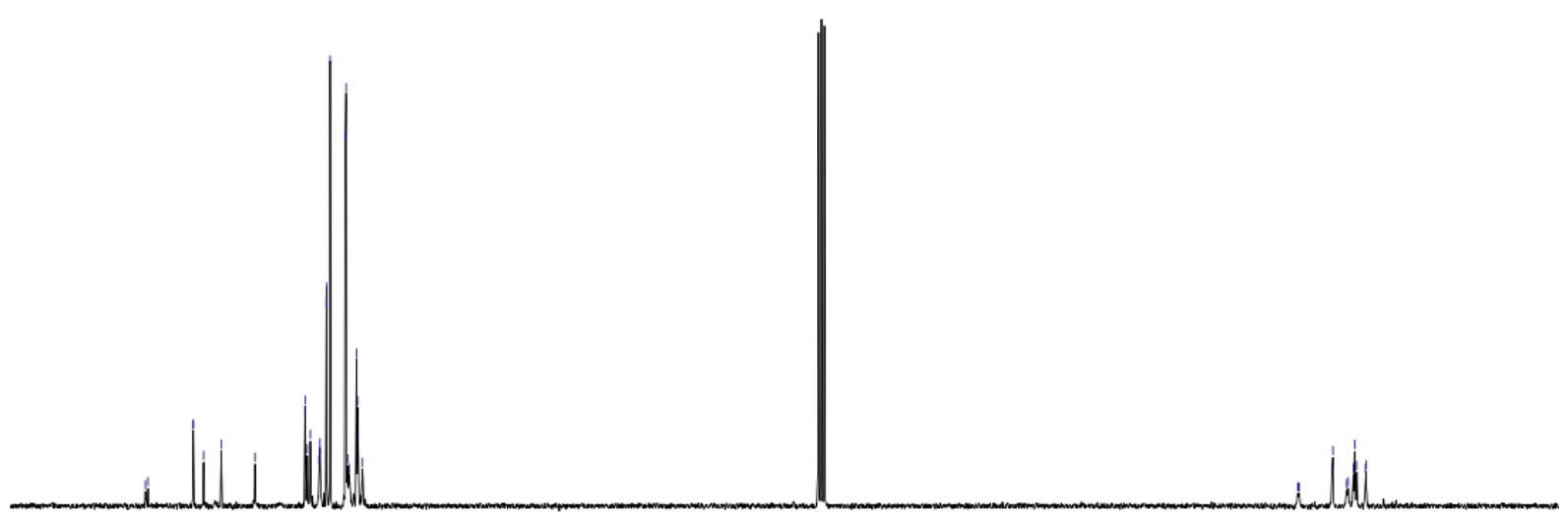

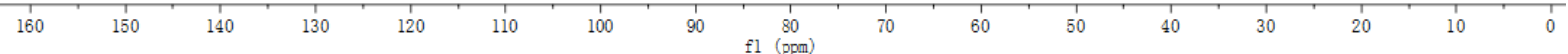
$S-70$ 
3ca | ${ }^{31} \mathrm{P}\left\{{ }^{1} \mathrm{H}\right\}$ NMR $\left(\mathrm{CDCl}_{3}, 162 \mathrm{MHz}\right)$

iे<smiles>O=[PH](O)c1ccccc1-c1ccccc1/C(=C/c1ccccc1)c1ccccc1</smiles>

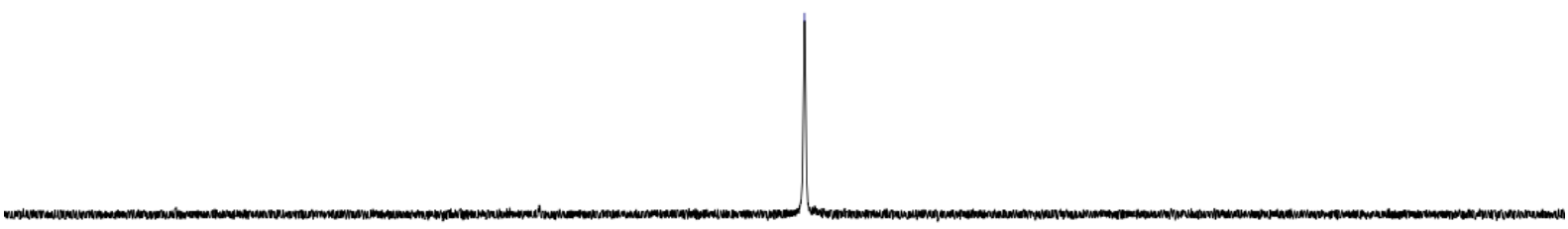

$\begin{array}{llllllllllllllllllllllllllllllll}150 & 140 & 130 & 120 & 110 & 100 & 90 & 80 & 70 & 60 & 50 & 40 & 30 & 20 & 10 & 0 & -10 & -20 & -30 & -40 & -50 & -60 & -70 & -80 & -90 & -100 & -110 & -120 & -130 & -140 & -150\end{array}$ 
3da| ${ }^{1} \mathrm{H} \mathrm{NMR}\left(\mathrm{CDCl}_{3}, 400 \mathrm{MHz}\right)$

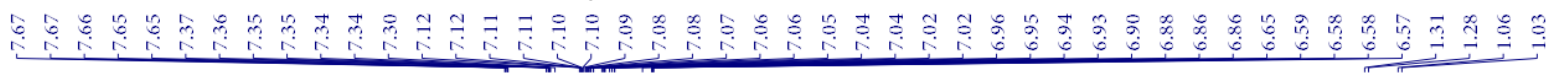
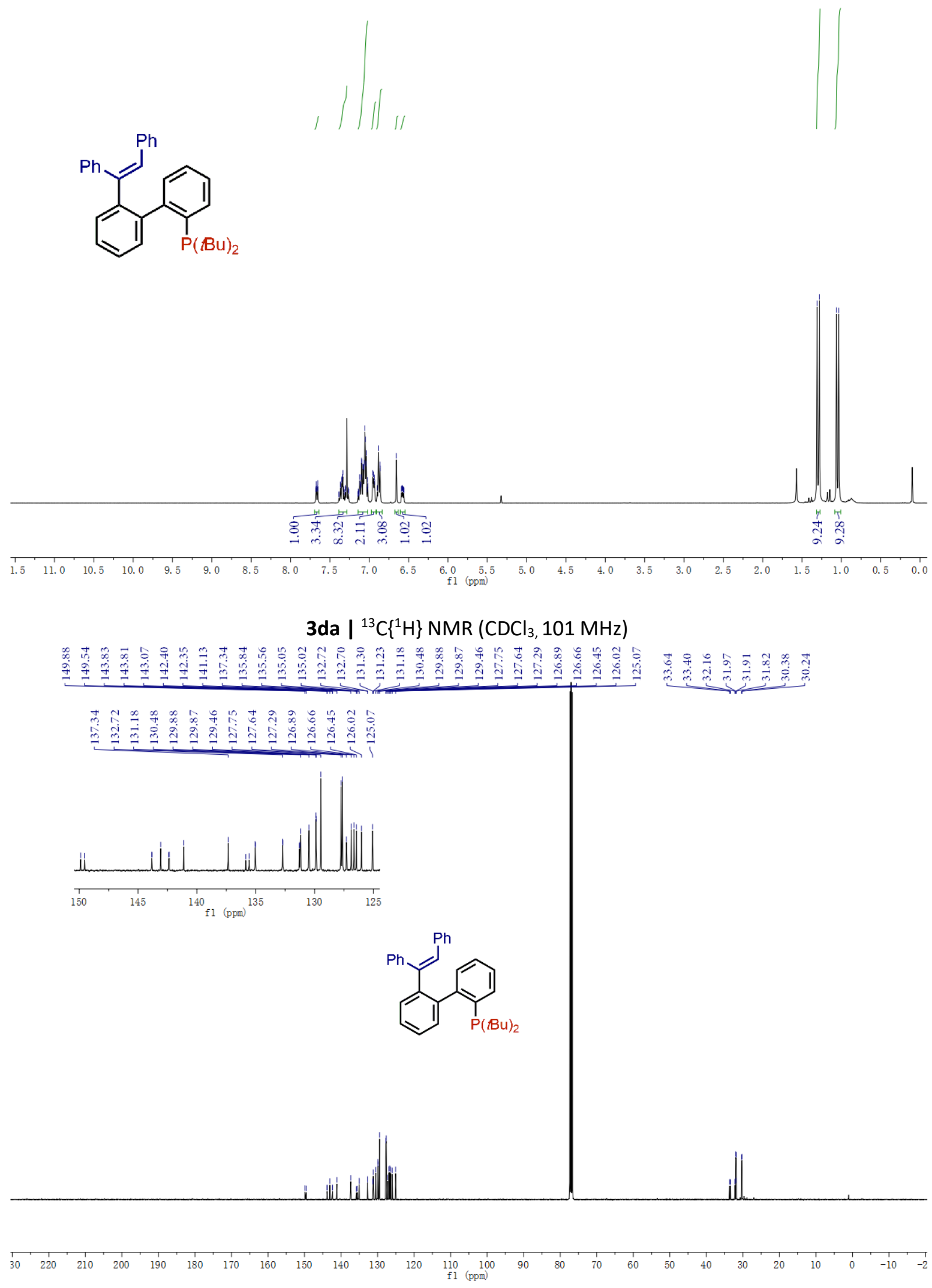

S - 72 
3da ${ }^{31} \mathrm{P}\left\{{ }^{1} \mathrm{H}\right\} \mathrm{NMR}\left(\mathrm{CDCl}_{3}, 162 \mathrm{MHz}\right)$

i<smiles>CCCCc1ccccc1-c1ccccc1C(=Cc1ccccc1)c1ccccc1</smiles>

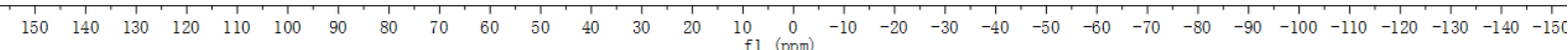


<smiles>Cc1cccc(C(=Cc2ccccc2)c2ccccc2)c1-c1ccccc1</smiles>
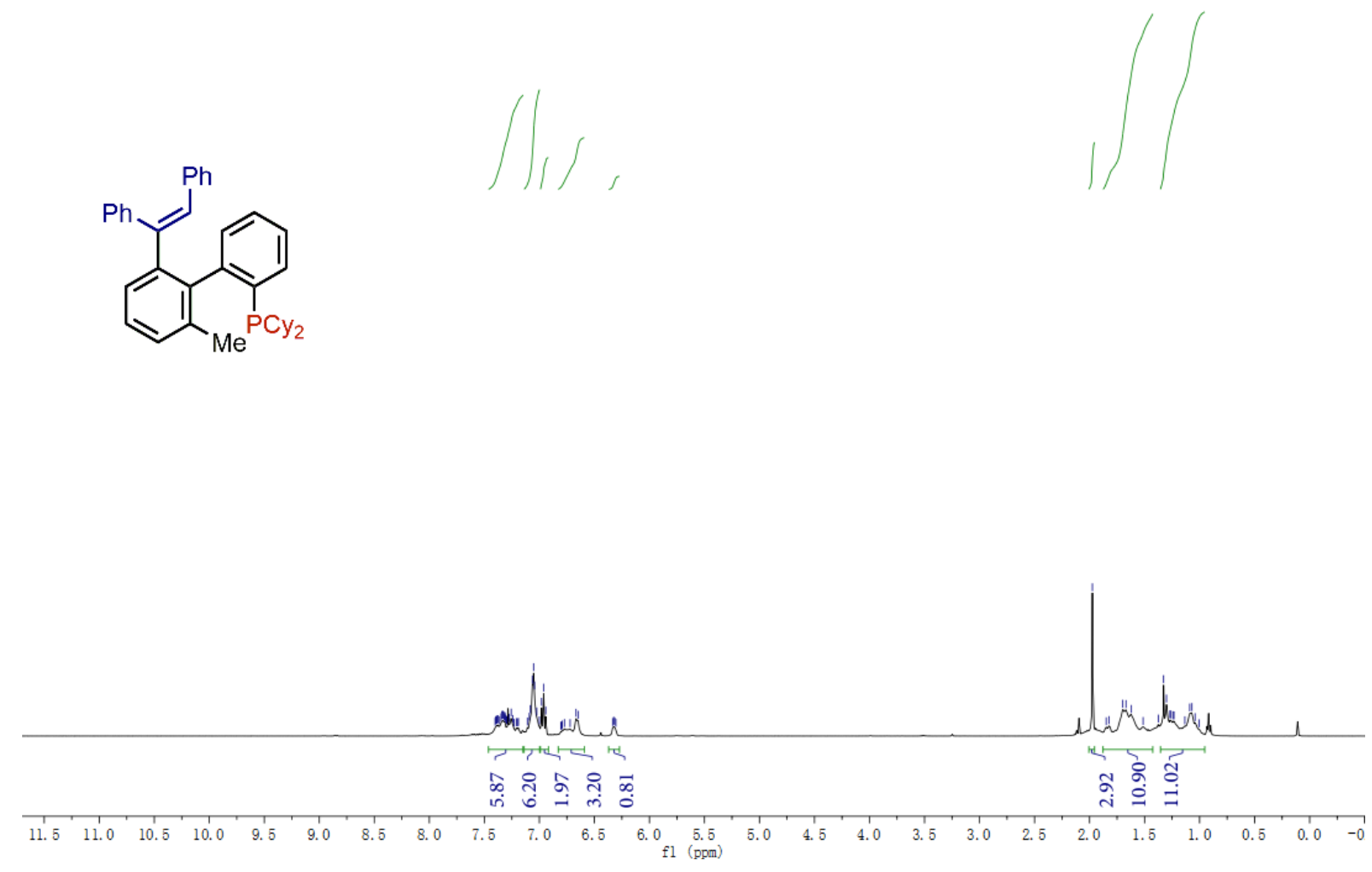

3ea | ${ }^{13} \mathrm{C}\left\{{ }^{1} \mathrm{H}\right\} \mathrm{NMR}\left(\mathrm{CDCl}_{3}, 101 \mathrm{MHz}\right)$

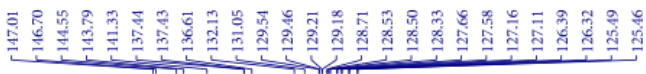

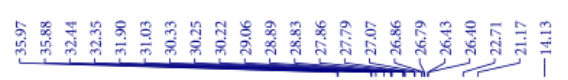

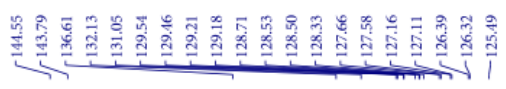
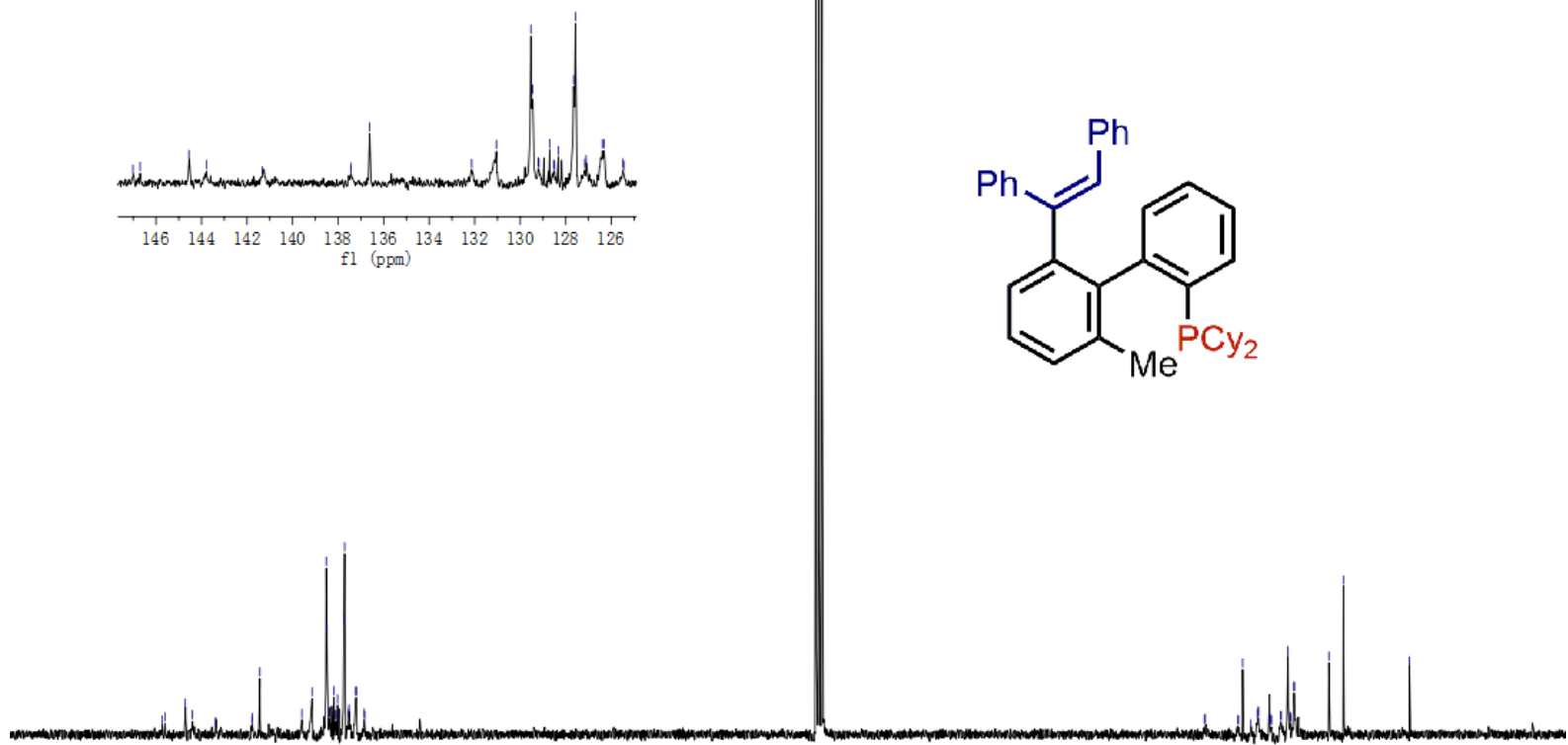

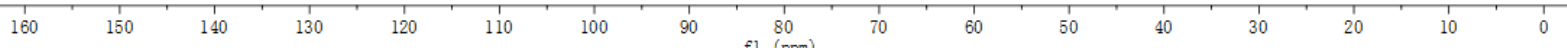


3ea | ${ }^{31} \mathrm{P}\left\{{ }^{1} \mathrm{H}\right\} \mathrm{NMR}\left(\mathrm{CDCl}_{3}, 162 \mathrm{MHz}\right)$<smiles>O=[N+]([O-])c1ccccc1-c1c(C(=Cc2ccccc2)c2ccccc2)cccc1[N+](=O)[O-]</smiles>

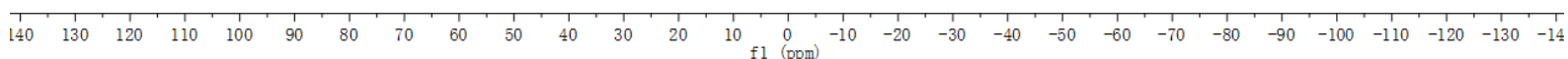


<smiles>CN(C)c1cccc(C(=Cc2ccccc2)c2ccccc2)c1-c1ccccc1</smiles>
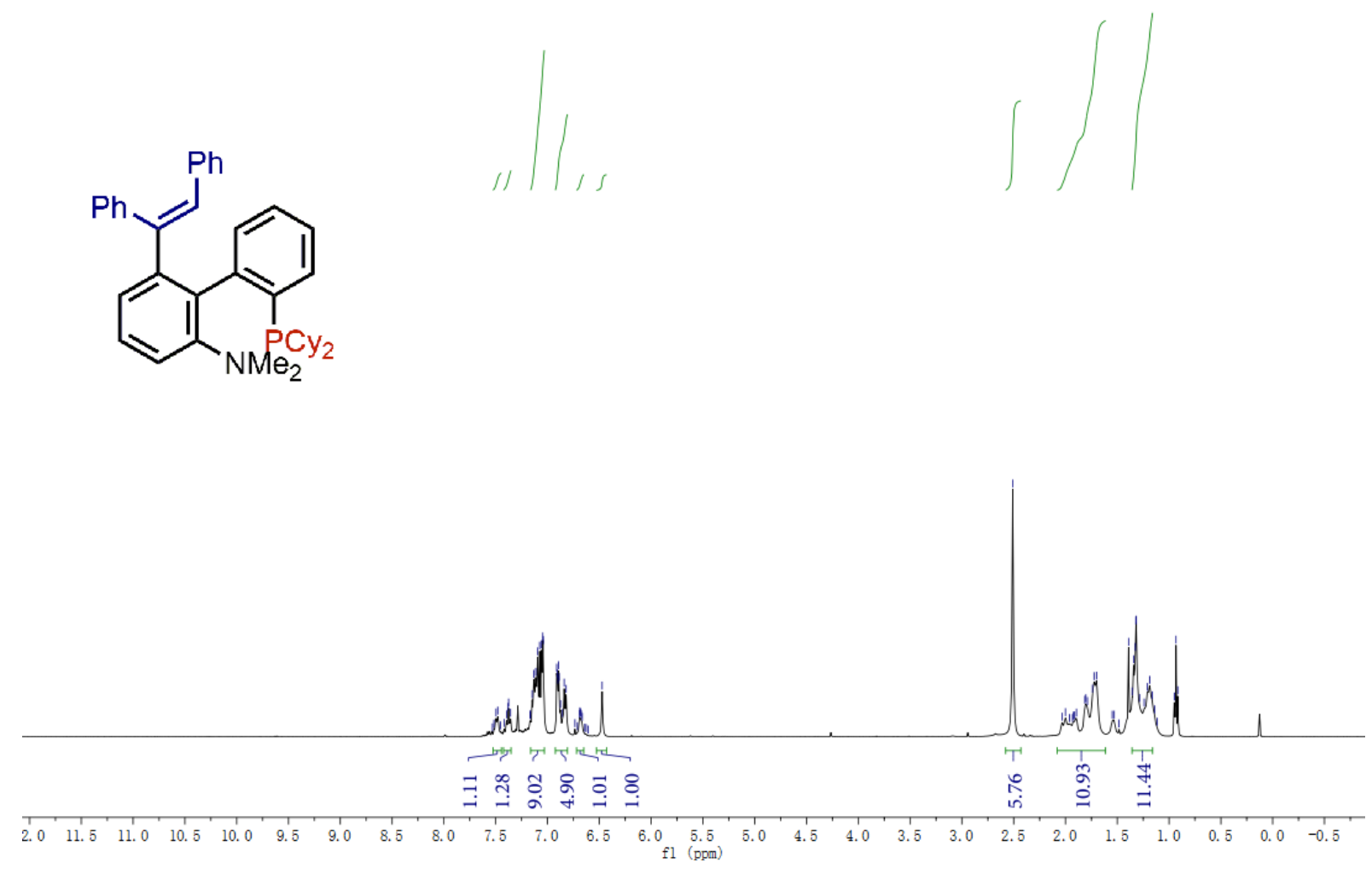

3fa $\mid{ }^{13} \mathrm{C}\left\{{ }^{1} \mathrm{H}\right\}$ NMR $\left(\mathrm{CDCl}_{3}, 101 \mathrm{MHz}\right)$

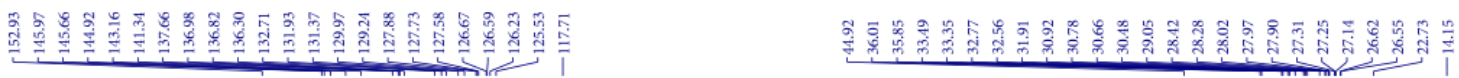<smiles>Cc1cccc(C(=Cc2ccccc2)c2ccccc2)c1-c1ccccc1N(C)C</smiles>

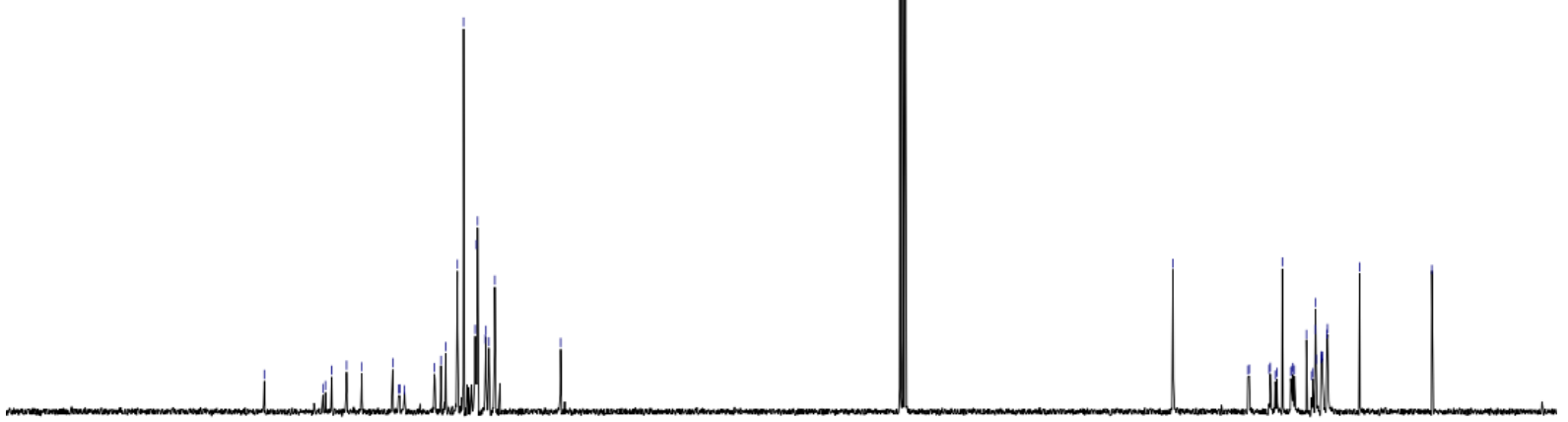

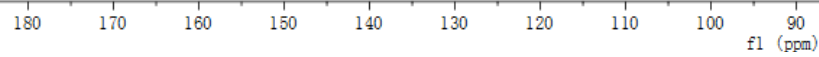


3fa $\mid{ }^{31} \mathrm{P}\left\{{ }^{1} \mathrm{H}\right\}$ NMR $\left(\mathrm{CDCl}_{3}, 162 \mathrm{MHz}\right)$

$\stackrel{\substack{0 \\ i}}{i}$<smiles>CN(C)c1cccc(C(=Cc2ccccc2)c2ccccc2)c1-c1ccccc1</smiles>

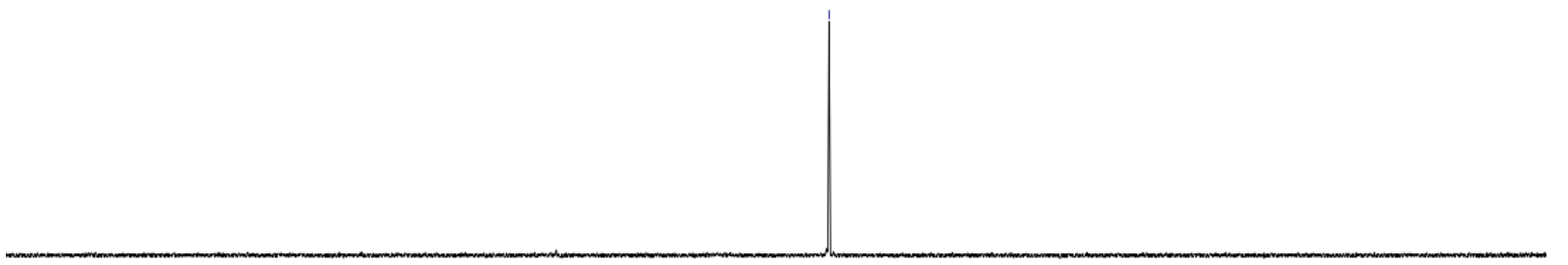

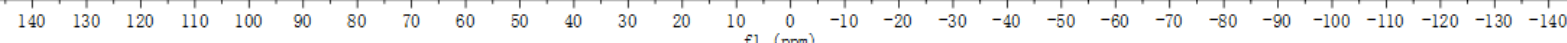


<smiles>CN(C)c1ccc(-c2ccccc2P(=O)(O)c2ccccc2)c(/C(=C/c2ccccc2)c2ccccc2)c1</smiles>
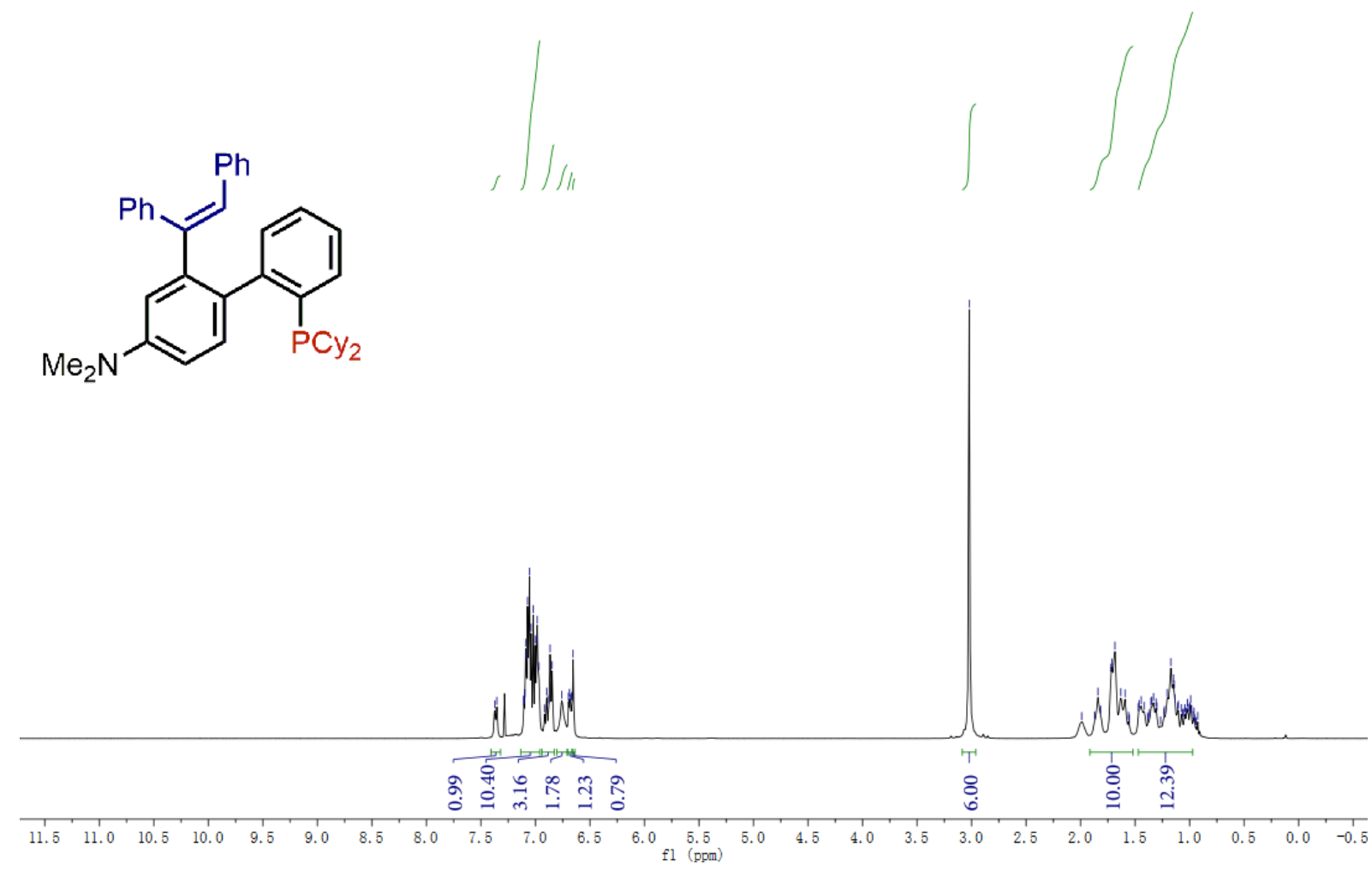

3ga | ${ }^{13} \mathrm{C}\left\{{ }^{1} \mathrm{H}\right\} \mathrm{NMR}\left(\mathrm{CDCl}_{3}, 101 \mathrm{MHz}\right)$

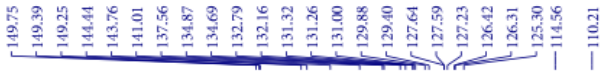

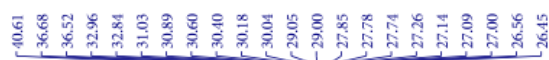<smiles>CN(C)c1ccc(-c2ccccc2P(=O)(O)c2ccccc2)c(/C(=C/c2ccccc2)c2ccccc2)c1</smiles>

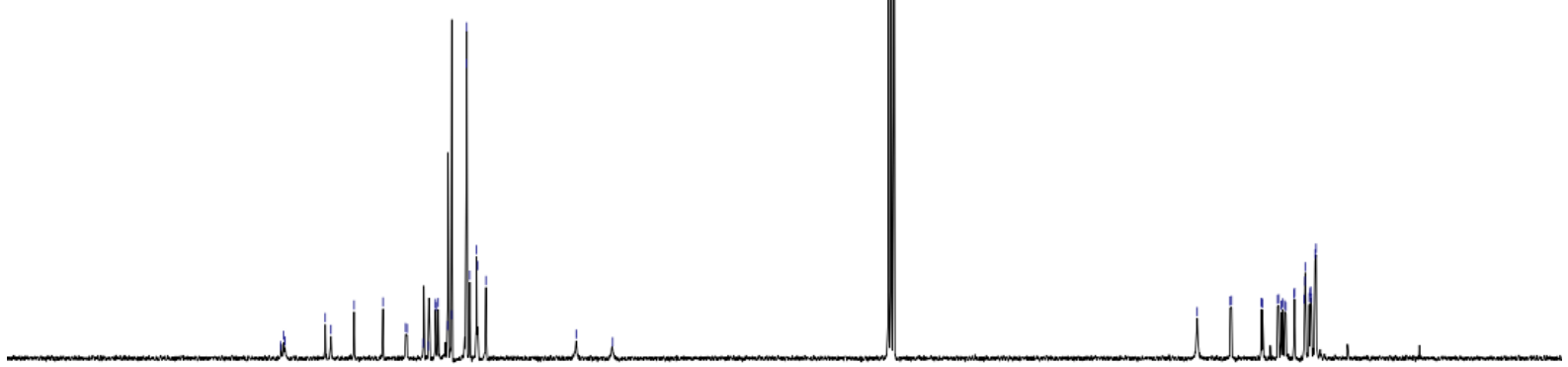

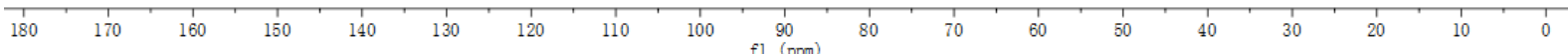


3ga | ${ }^{31} \mathrm{P}\left\{{ }^{1} \mathrm{H}\right\} \mathrm{NMR}\left(\mathrm{CDCl}_{3}, 162 \mathrm{MHz}\right)$

$\stackrel{\circ}{i}$

(l)

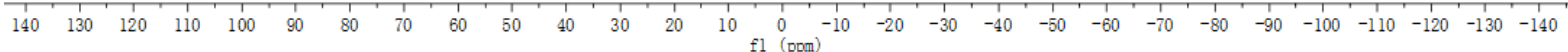


3ha $\mid{ }^{1} \mathrm{H} \mathrm{NMR}\left(\mathrm{CDCl}_{3}, 400 \mathrm{MHz}\right)$

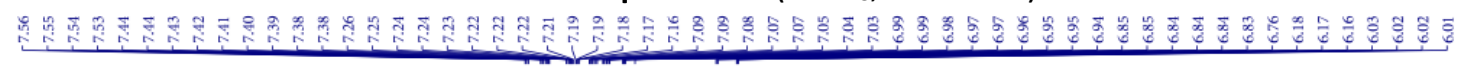<smiles></smiles>
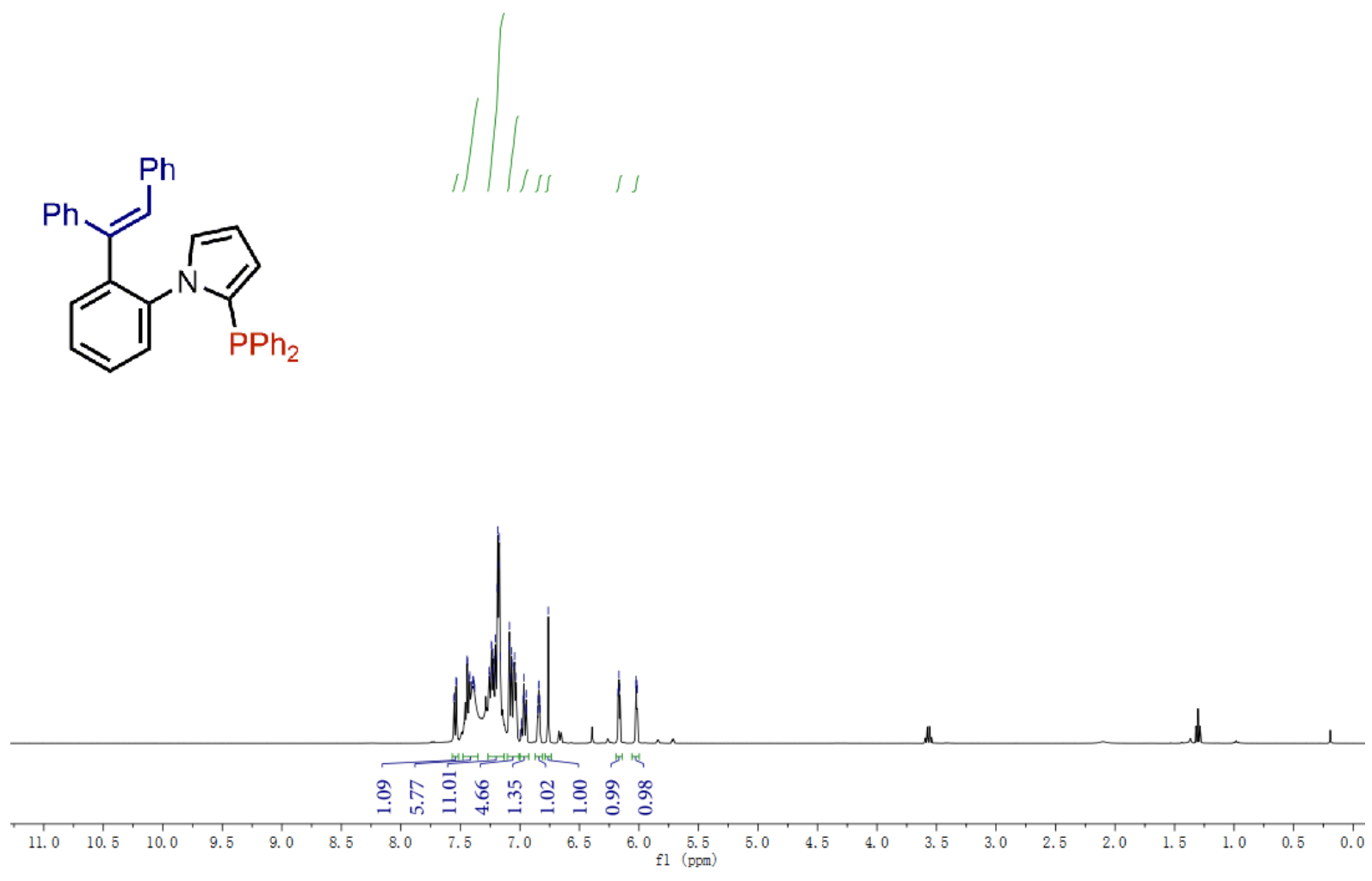

3ha ${ }^{13} \mathrm{C}\left\{{ }^{1} \mathrm{H}\right\} \mathrm{NMR}\left(\mathrm{CDCl}_{3}, 101 \mathrm{MHz}\right)$

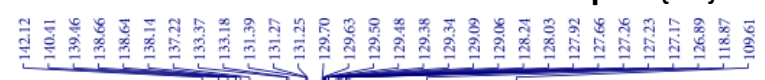

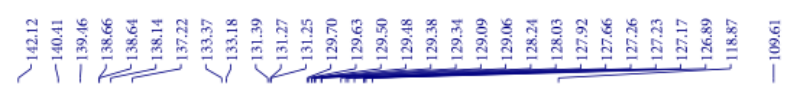

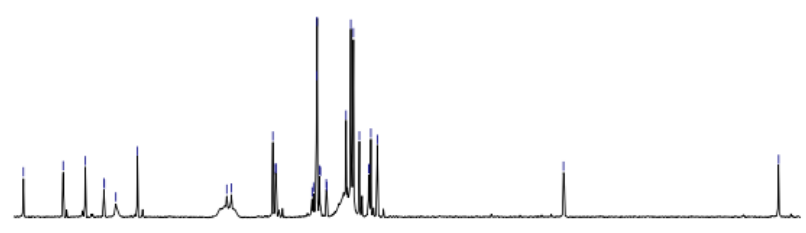

$\begin{array}{lllllllllllllllllllllll}142 & 140 & 138 & 136 & 134 & 132 & 130 & 128 & 126 & 124 & 122 & 1 & 1 & 1 & 1 & 1 & 1 & 1 & 1 & 1 & 1 & 1 & 1\end{array}$
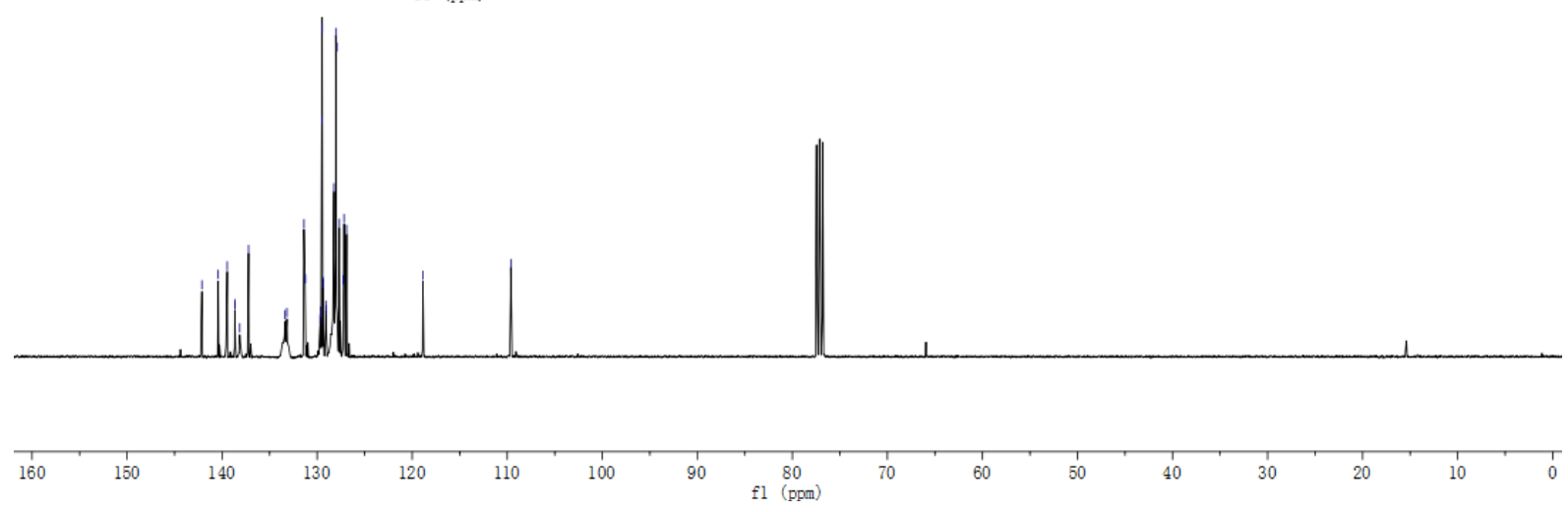

$S-80$ 
3ha | ${ }^{31} \mathrm{P}\left\{{ }^{1} \mathrm{H}\right\} \mathrm{NMR}\left(\mathrm{CDCl}_{3}, 162 \mathrm{MHz}\right)$

i<smiles>c1ccc(-c2ccccc2-n2cccc2-c2ccccc2)cc1</smiles>

$\begin{array}{llllllllllllllllllllllllllllllll}50 & 140 & 130 & 120 & 110 & 100 & 90 & 80 & 70 & 60 & 50 & 40 & 30 & 20 & 10 & 0 & -10 & -1 & -30 & -40 & -50 & -60 & -70 & -80 & -90 & -100 & -110 & -120 & -130 & -140 & -15\end{array}$ 
<smiles>COc1ccccc1-c1c(/C(=C/c2ccccc2)c2ccccc2)c2ccccc2n1C</smiles>
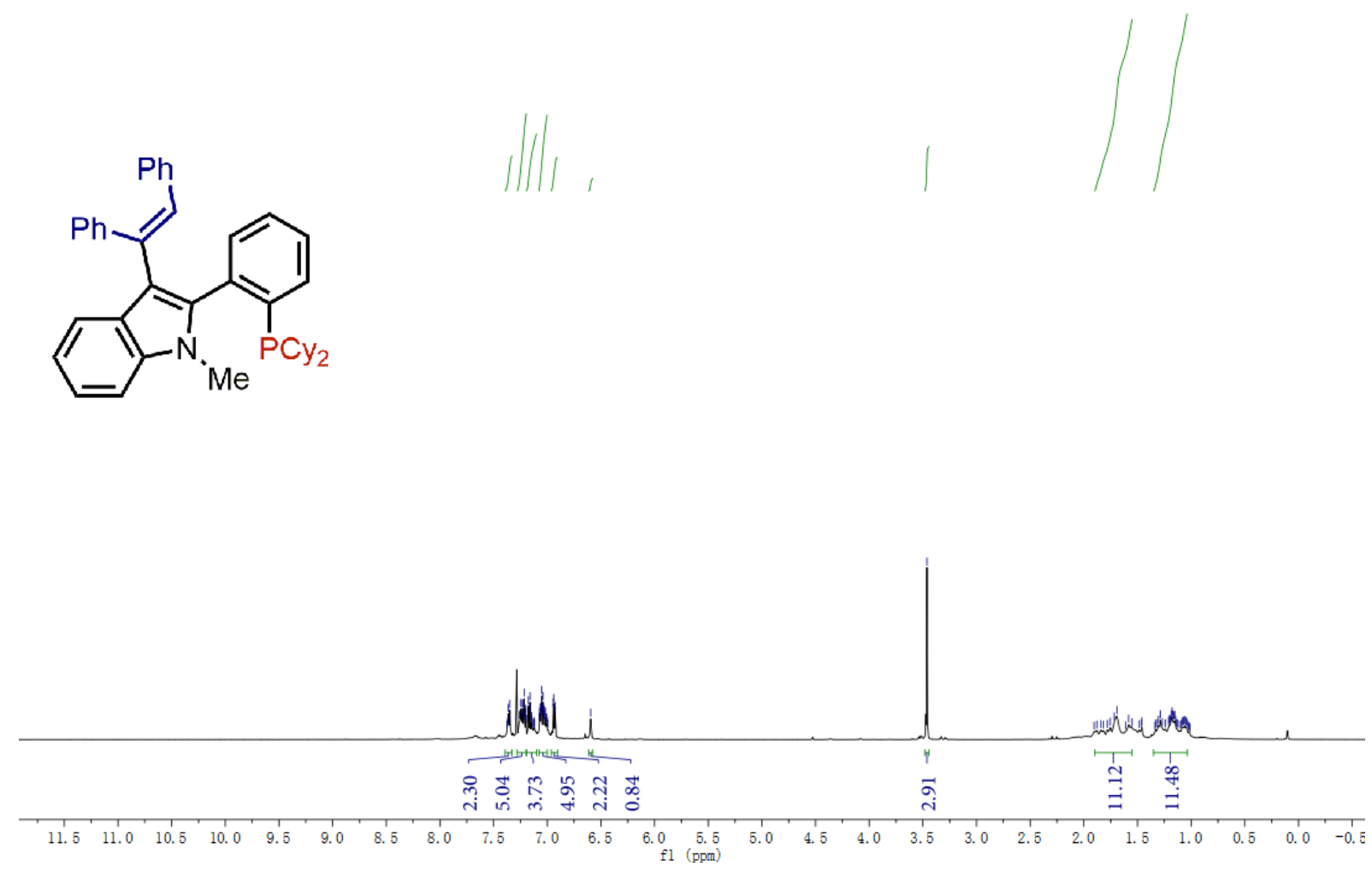

3ia | ${ }^{13} \mathrm{C}\left\{{ }^{1} \mathrm{H}\right\}$ NMR $\left(\mathrm{CDCl}_{3}, 101 \mathrm{MHz}\right)$

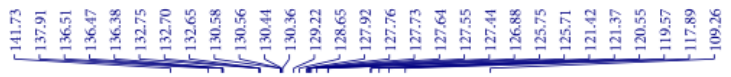

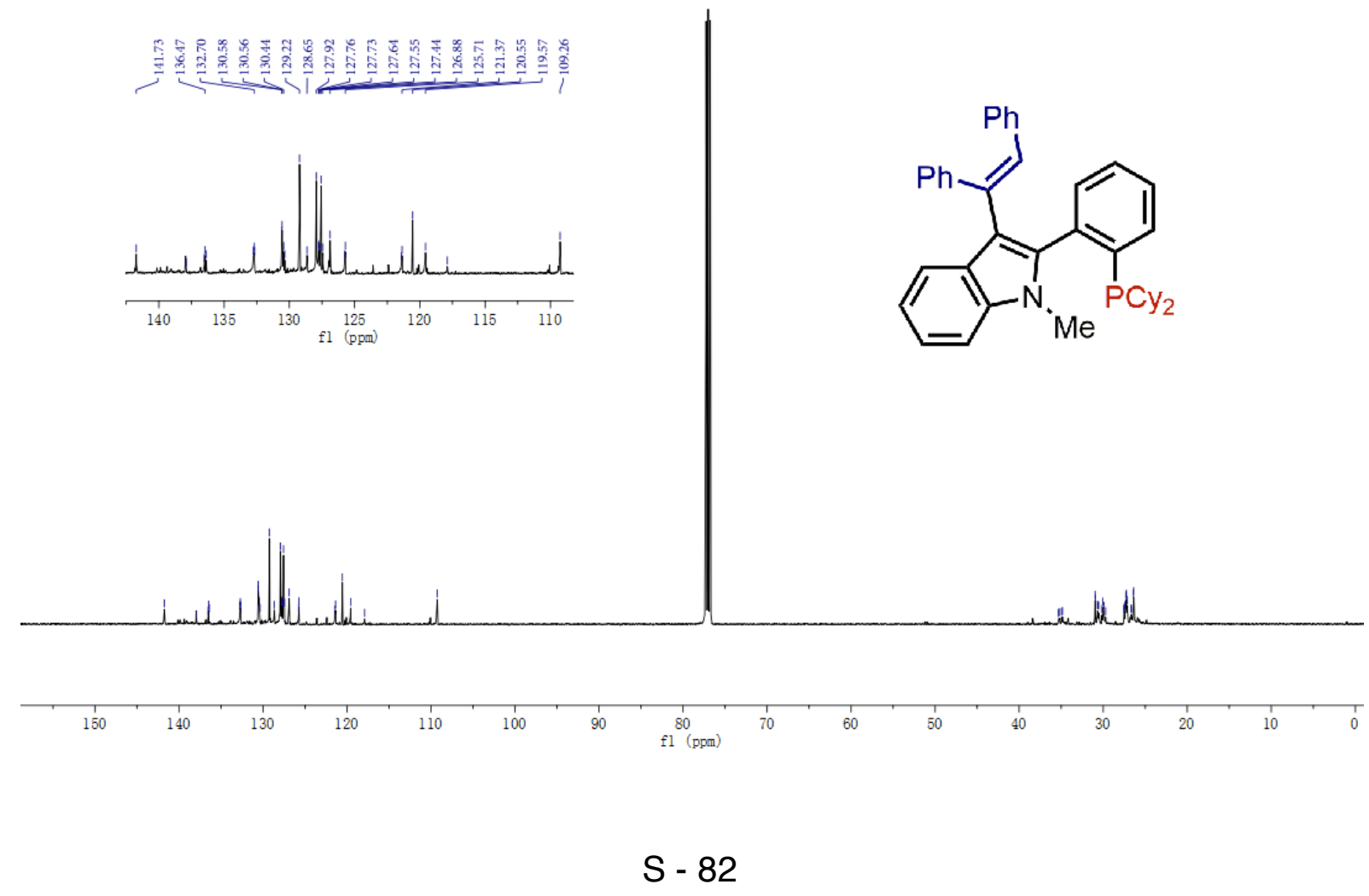


3ia | ${ }^{31} \mathrm{P}\left\{{ }^{1} \mathrm{H}\right\} \mathrm{NMR}\left(\mathrm{CDCl}_{3}, 162 \mathrm{MHz}\right)$

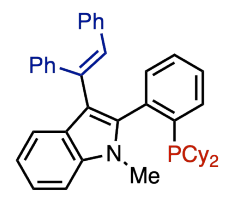

$\underset{\substack{0 \\ i}}{i}$

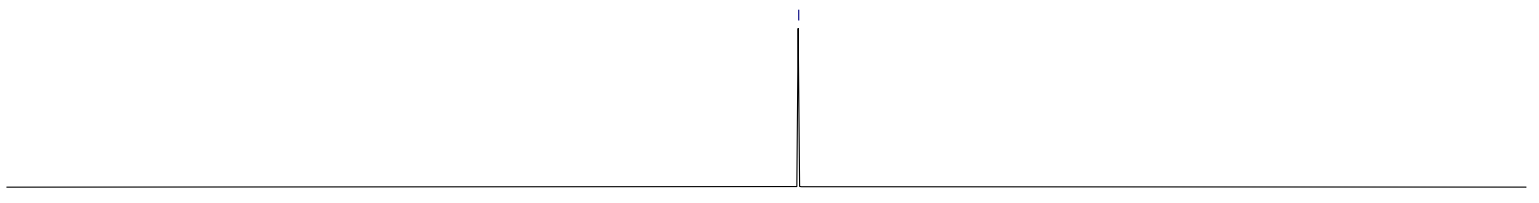

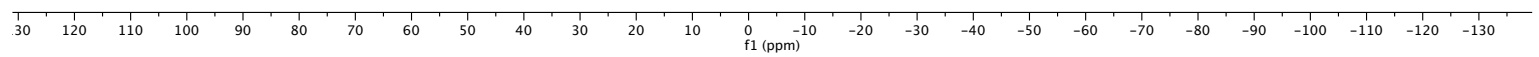


<smiles>CNc1cccc(C(=Cc2ccc(Br)cc2)c2ccc(Br)cc2)c1-c1ccccc1[PH](C)(=O)=O</smiles>
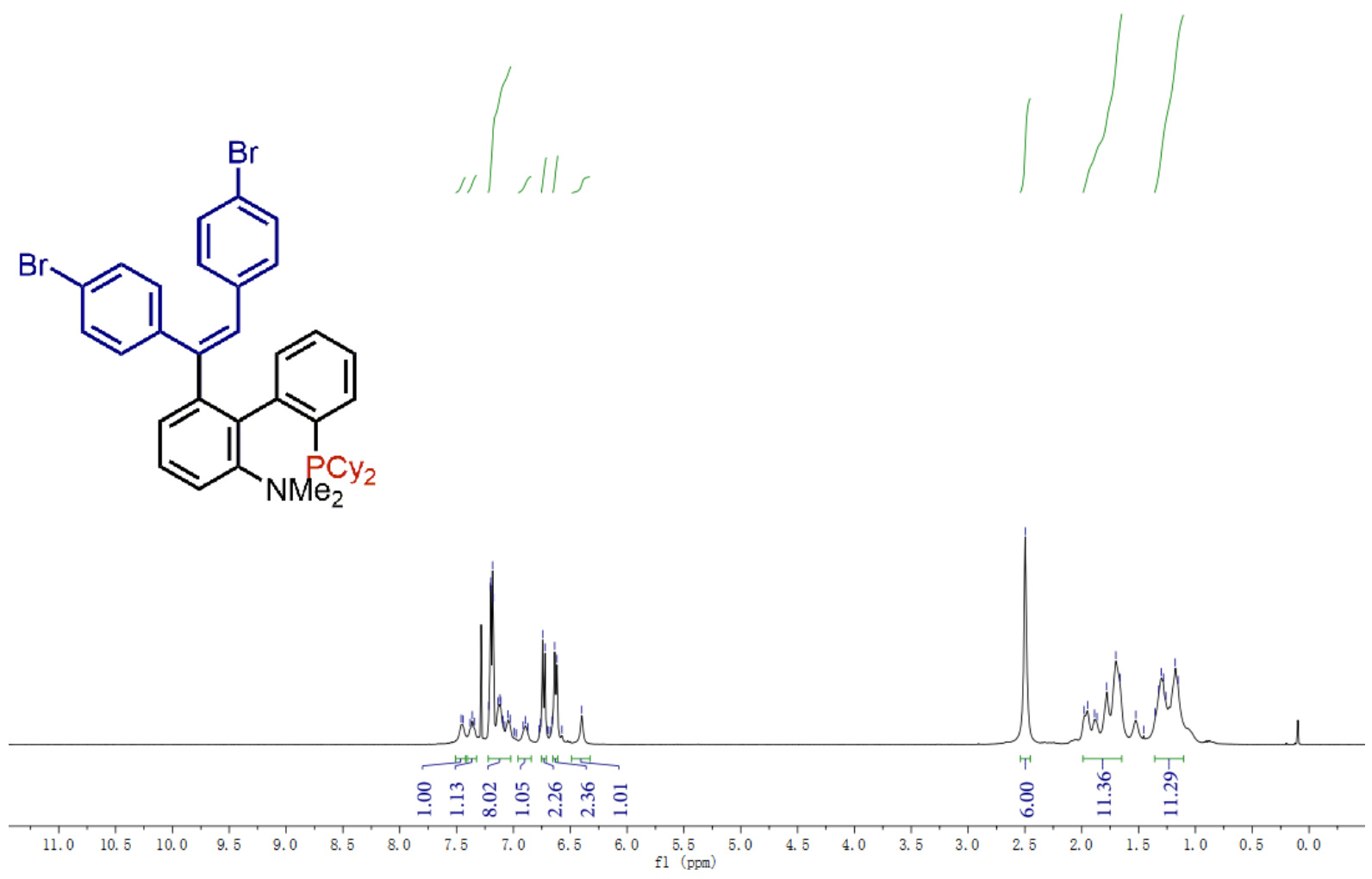

$3 \mathrm{fb} \mid{ }^{13} \mathrm{C}\left\{{ }^{1} \mathrm{H}\right\}$ NMR $\left(\mathrm{CDCl}_{3}, 101 \mathrm{MHz}\right)$

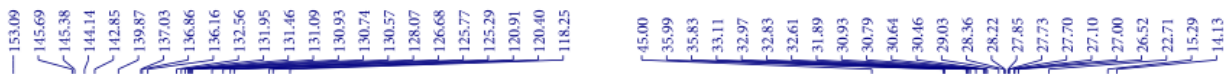<smiles>CNc1cccc(C(=Cc2ccc(Br)cc2)c2ccc(Br)cc2)c1-c1ccccc1C(C)(C)C</smiles> 
3fb $\mid{ }^{31} \mathrm{P}\left\{{ }^{1} \mathrm{H}\right\} \mathrm{NMR}\left(\mathrm{CDCl}_{3}, 162 \mathrm{MHz}\right)$

$\stackrel{\circ}{\infty}$<smiles>CNc1cccc(C(=Cc2ccc(Br)cc2)c2ccc(Br)cc2)c1-c1ccccc1[PH](C)(=O)=O</smiles>

${ }_{140}^{140} \quad \begin{array}{llllllllllllllllllllllllllllllllll}120 & 110 & 100 & 90 & 80 & 70 & 60 & 50 & 40 & 30 & 20 & 10 & 0 & -10 & -20 & -30 & -40 & -50 & -60 & -70 & -80 & -90 & -100 & -110 & -120 & -130 & -14\end{array}$ 
<smiles>O=C(O)c1ccccc1-c1ccccc1C(=Cc1ccc(Br)cc1)c1ccc(Br)cc1</smiles>
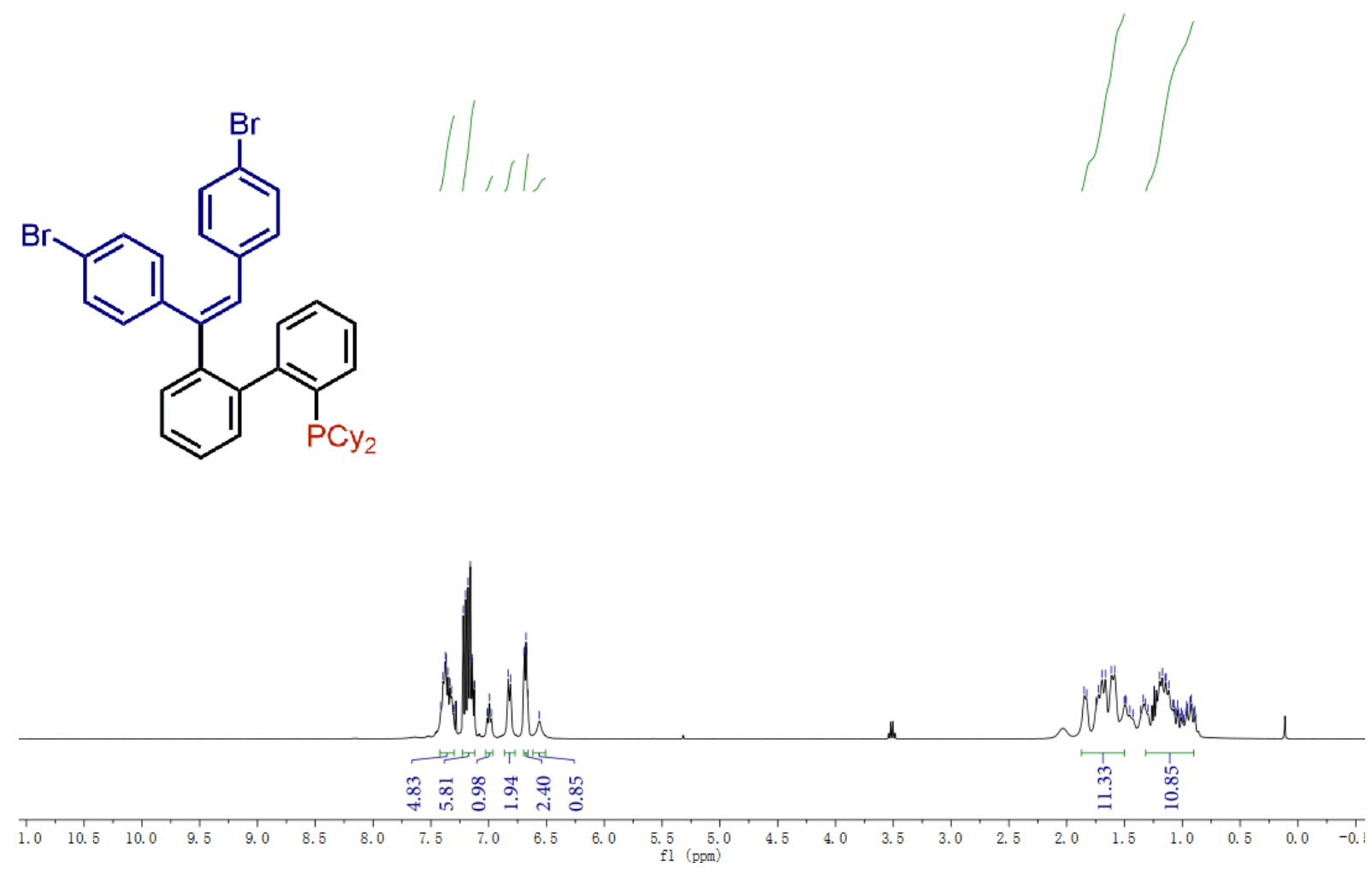

3ab $\mid{ }^{13} \mathrm{C}\left\{{ }^{1} \mathrm{H}\right\}$ NMR $\left(\mathrm{CDCl}_{3}, 101 \mathrm{MHz}\right)$

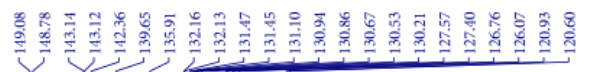

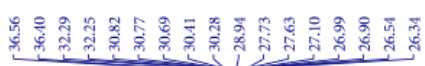

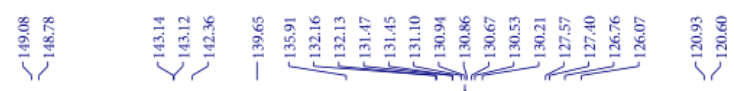
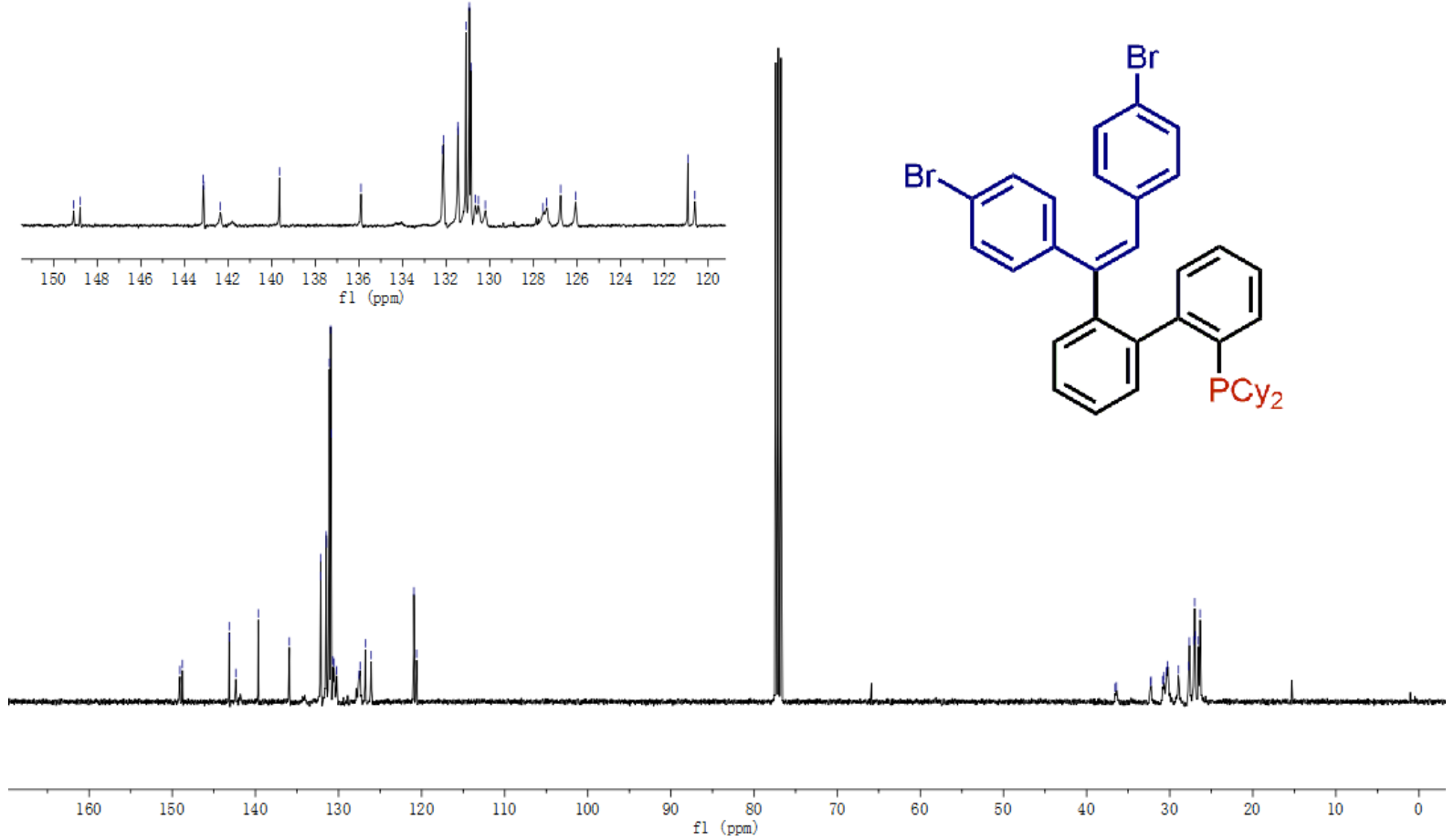
3ab | ${ }^{31} \mathrm{P}\left\{{ }^{1} \mathrm{H}\right\} \mathrm{NMR}\left(\mathrm{CDCl}_{3}, 162 \mathrm{MHz}\right)$

$\stackrel{n}{\stackrel{1}{i}}$<smiles>CCOc1ccccc1-c1ccccc1C(=Cc1ccc(Br)cc1)c1ccc(Br)cc1</smiles>

$\begin{array}{llllllllllllllllllllllllllllll}140 & 130 & 120 & 110 & 100 & 90 & 80 & 70 & 60 & 50 & 40 & 30 & 20 & 10 & 0 & -10 & -20 & -30 & -40 & -50 & -60 & -70 & -80 & -90 & -100 & -110 & -120 & -130 & -140\end{array}$ 
<smiles>Cc1ccc(C=C(c2ccc(C)cc2)c2ccccc2-c2ccccc2P(C)(=O)OCc2ccccc2)cc1</smiles>
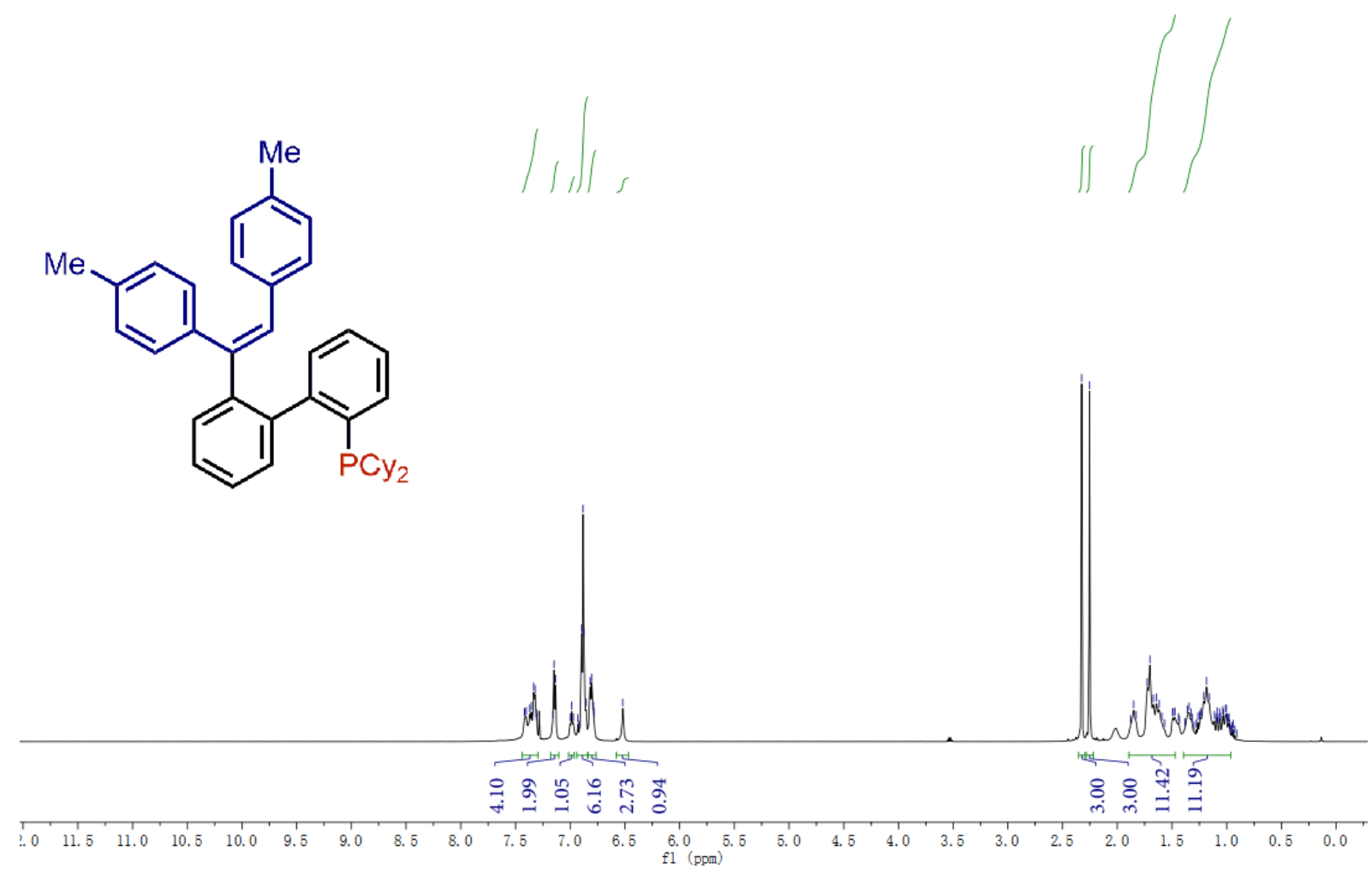

3ac $\mid{ }^{13} \mathrm{C}\left\{{ }^{1} \mathrm{H}\right\} \mathrm{NMR}\left(\mathrm{CDCl}_{3}, 101 \mathrm{MHz}\right)$

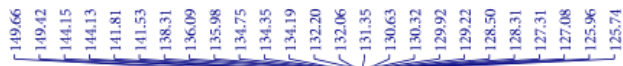

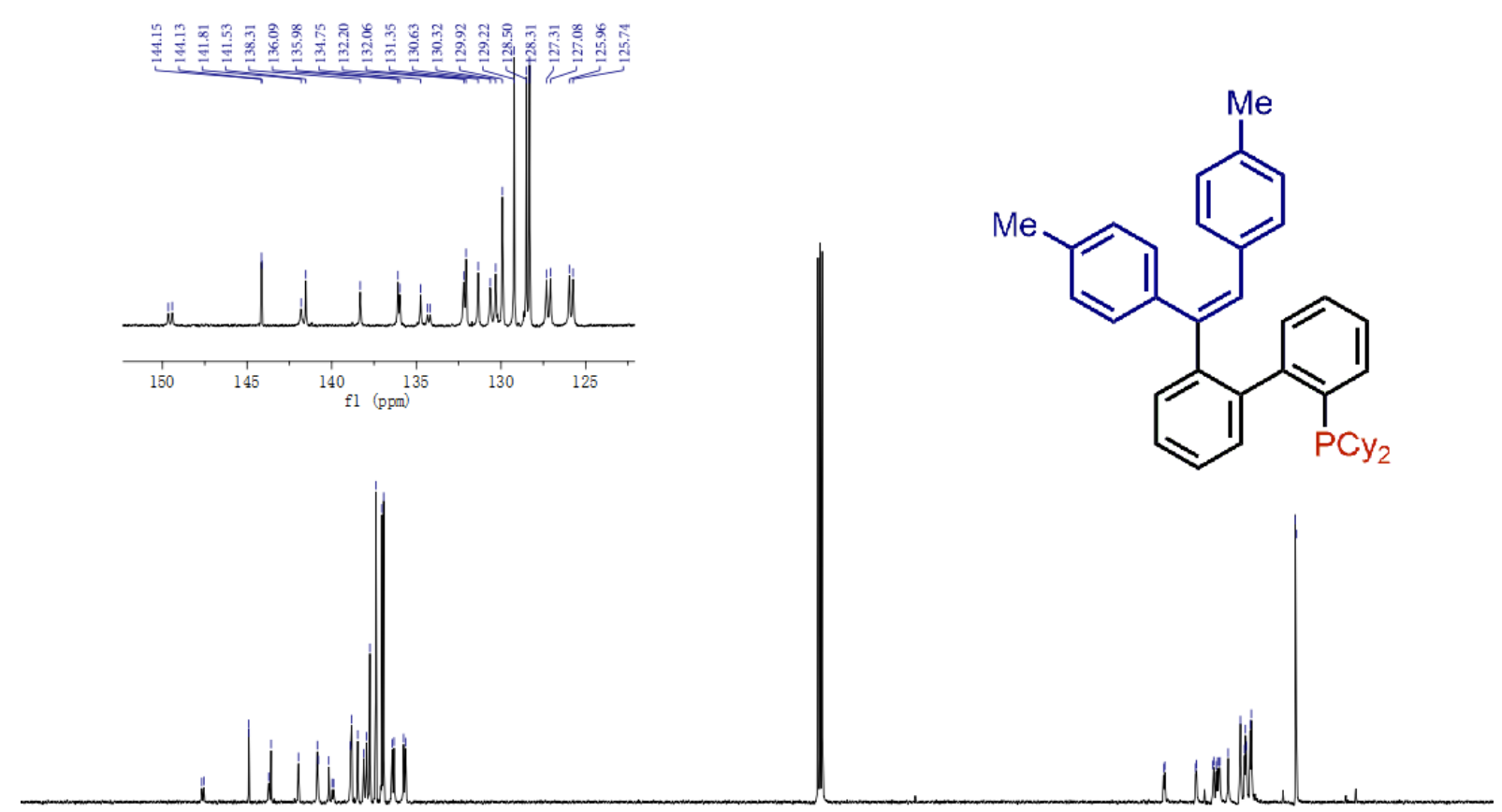

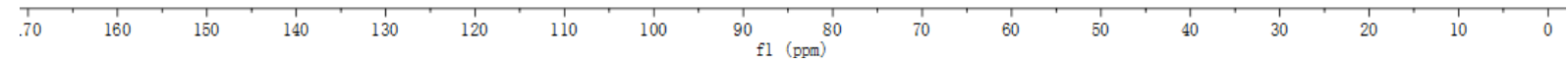


3ac $\mid{ }^{31} \mathrm{P}\left\{{ }^{1} \mathrm{H}\right\} \mathrm{NMR}\left(\mathrm{CDCl}_{3}, 162 \mathrm{MHz}\right)$<smiles>CCCCc1ccccc1-c1ccccc1C(=Cc1ccc(C)cc1)c1ccc(C)cc1</smiles>

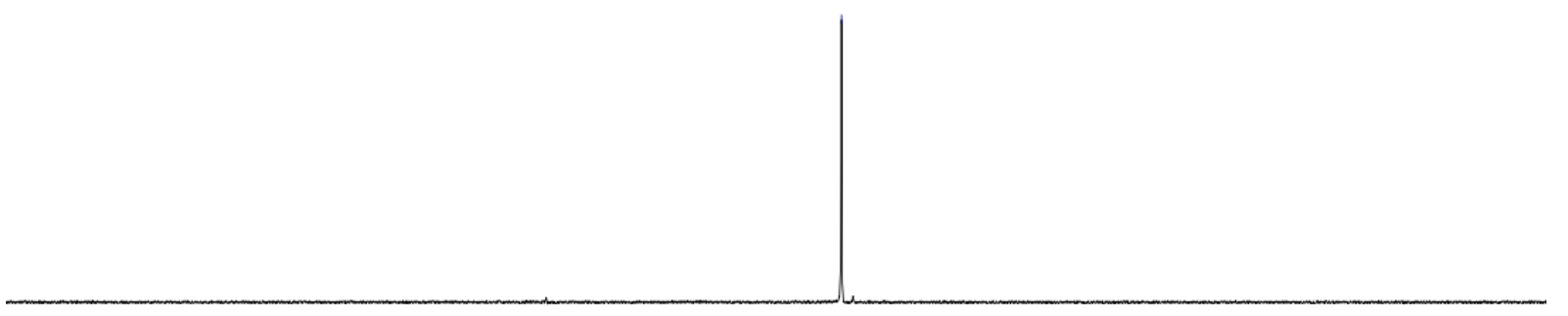

$\begin{array}{lllllllllllllllllllllllllllllllll}140 & 130 & 120 & 110 & 100 & 90 & 80 & 70 & 60 & 50 & 40 & 30 & 20 & 10 & 0 & -10 & -20 & -30 & -40 & -50 & -60 & -70 & -80 & -90 & -100 & -110 & -120 & -130 & -140\end{array}$ 

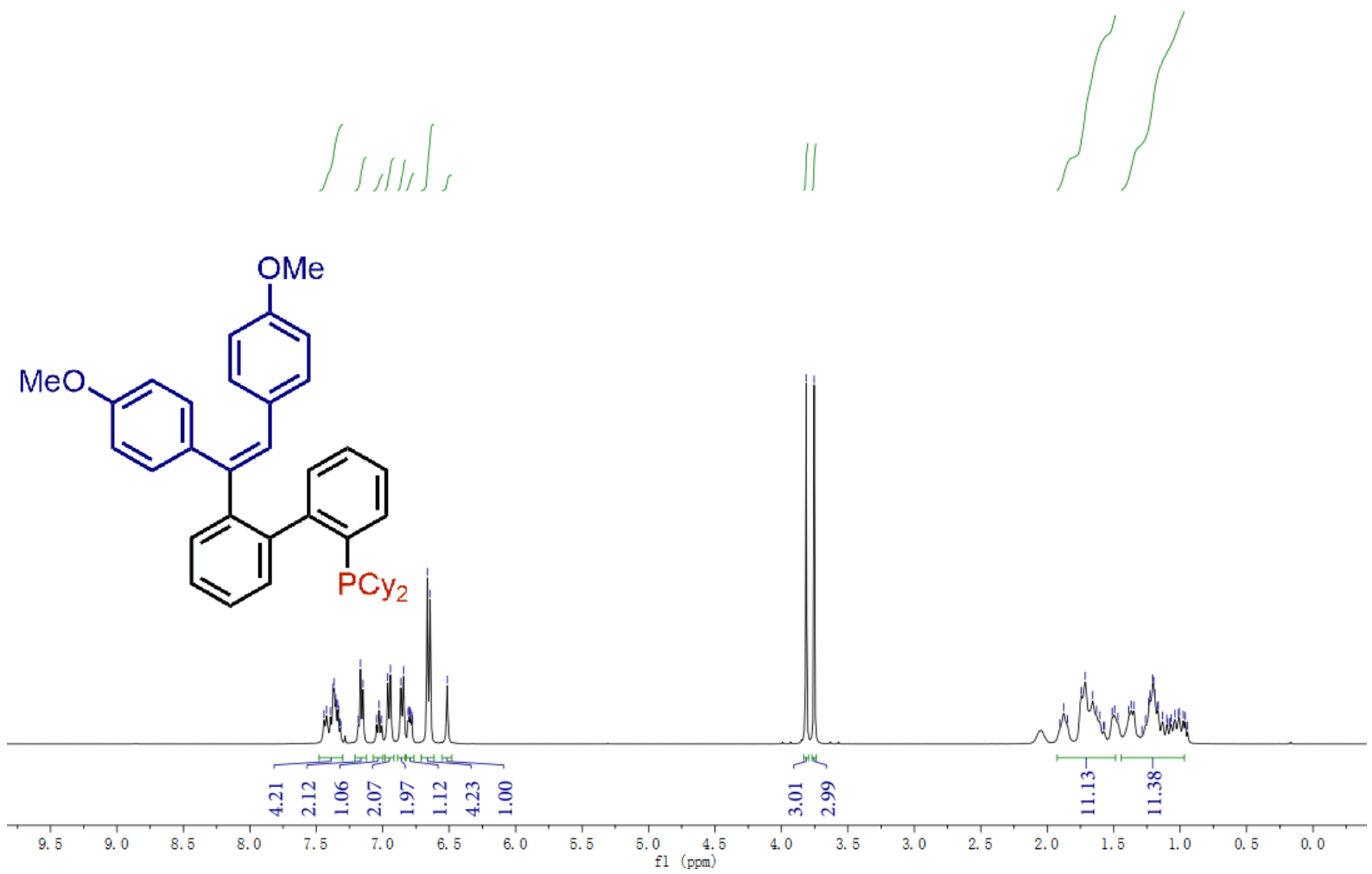

3ad $\mid{ }^{13} \mathrm{C}\left\{{ }^{1} \mathrm{H}\right\}$ NMR $\left(\mathrm{CDCl}_{3}, 101 \mathrm{MHz}\right)$

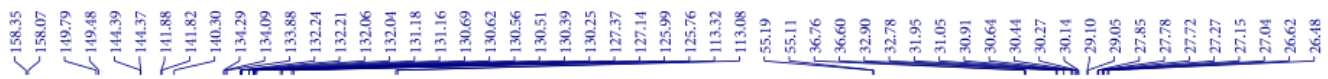<smiles>COc1ccc(C=C(c2ccc(OC)cc2)c2ccccc2-c2ccccc2P(C)(=O)c2ccccc2)cc1</smiles>

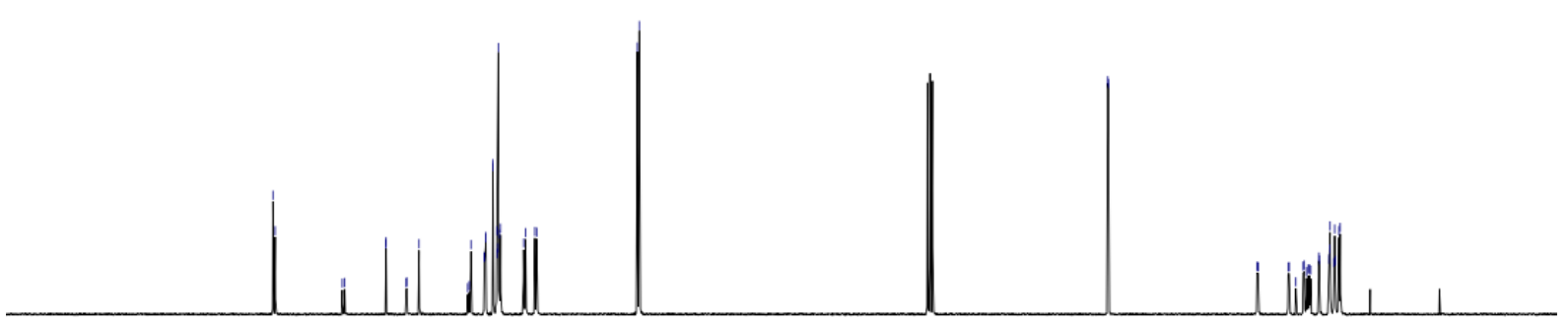

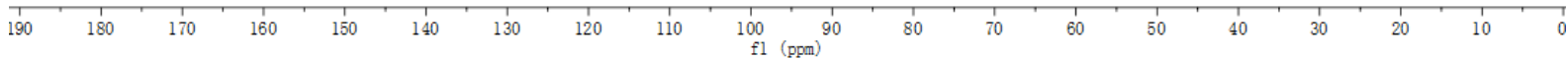

$$
\text { S - } 90
$$


3ad | ${ }^{31} \mathrm{P}\left\{{ }^{1} \mathrm{H}\right\} \mathrm{NMR}\left(\mathrm{CDCl}_{3}, 162 \mathrm{MHz}\right)$

iे<smiles>COc1ccc(/C=C(/c2ccc(OC)cc2)c2ccccc2-c2ccccc2P(C)(=O)c2ccccc2)cc1</smiles>

$\begin{array}{llllllllllllllllllllllllllllll}140 & 130 & 120 & 110 & 100 & 90 & 80 & 70 & 60 & 50 & 40 & 30 & 20 & 10 & 0 & -10 & -20 & -30 & -40 & -50 & -60 & -70 & -80 & -90 & -100 & -110 & -120 & -130 & -140\end{array}$ 


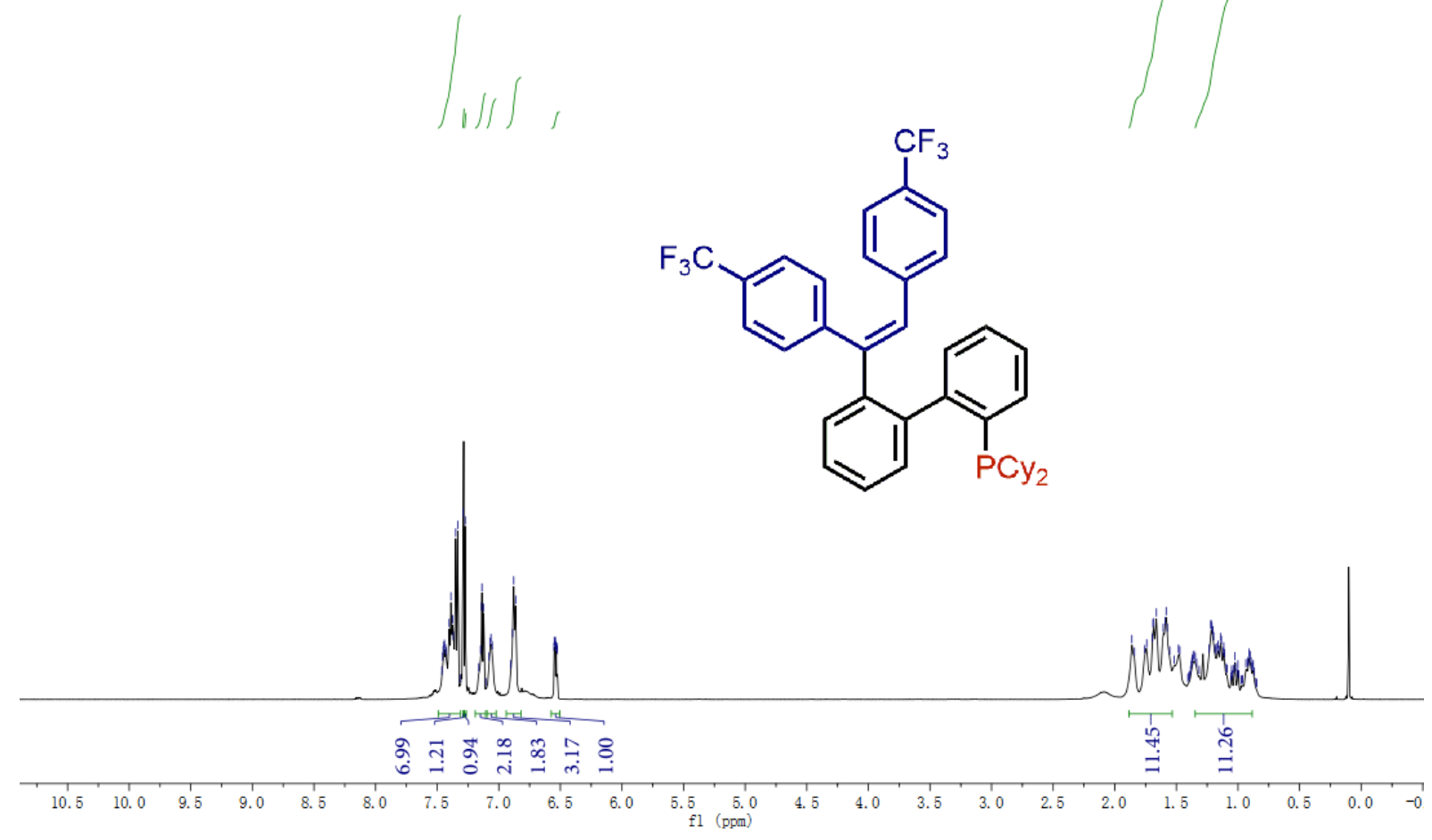

3ae $\mid{ }^{13} \mathrm{C}\left\{{ }^{1} \mathrm{H}\right\}$ NMR $\left(\mathrm{CDCl}_{3}, 101 \mathrm{MHz}\right)$

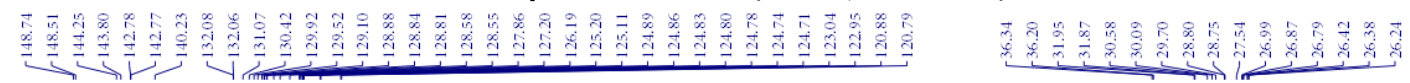

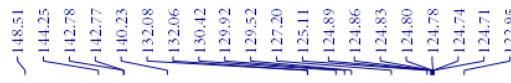
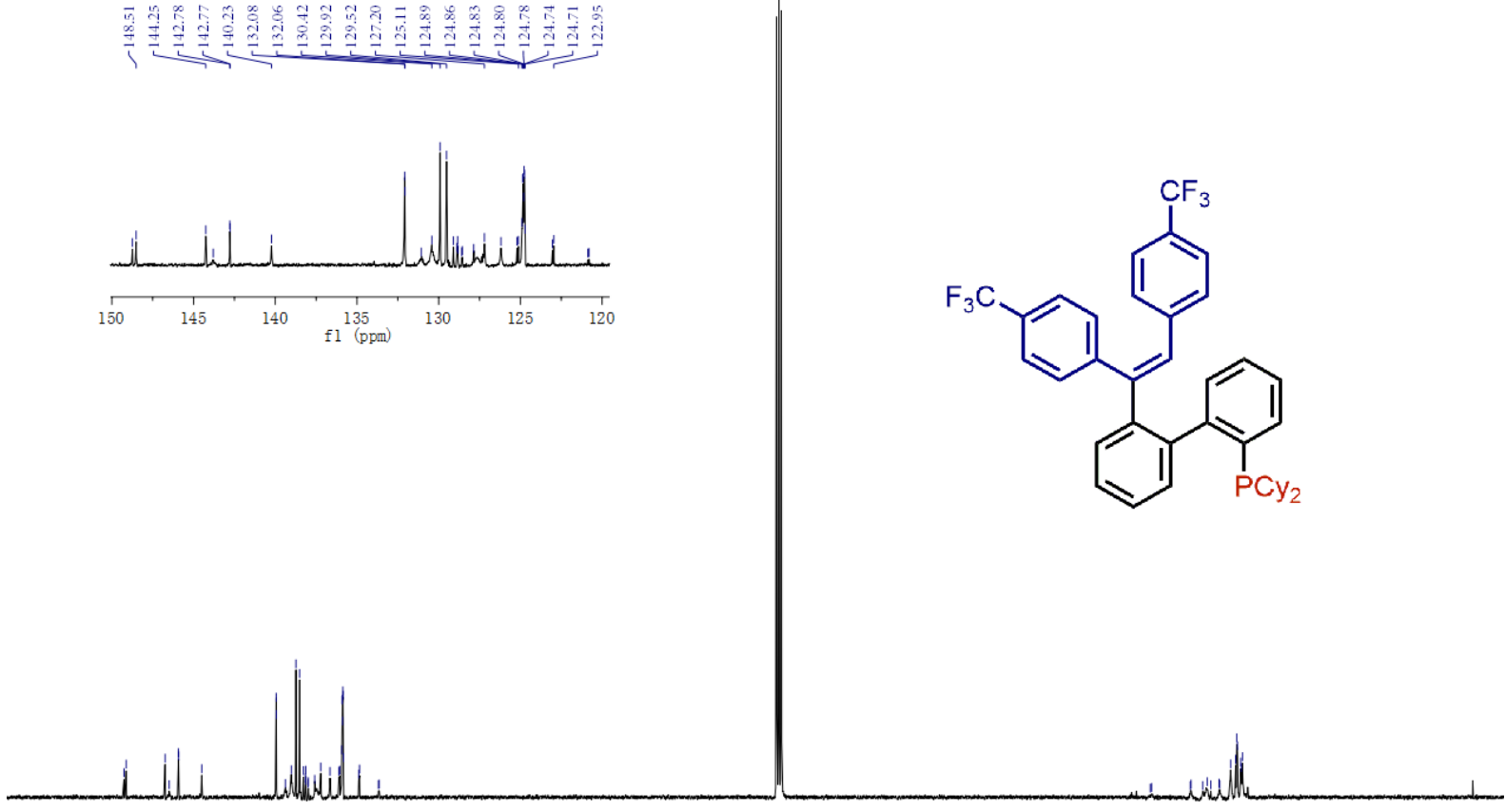

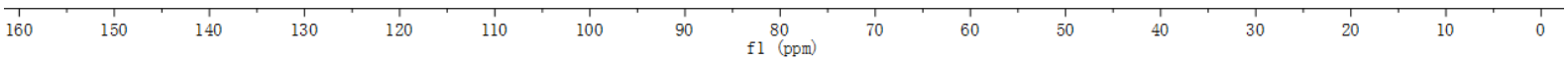


3ae | ${ }^{31} \mathrm{P}\left\{{ }^{1} \mathrm{H}\right\} \operatorname{NMR}\left(\mathrm{CDCl}_{3}, 162 \mathrm{MHz}\right)$<smiles>CCCCc1ccccc1-c1ccccc1C(=Cc1ccc(C(F)(F)F)cc1)c1ccc(C(F)(F)F)cc1</smiles>

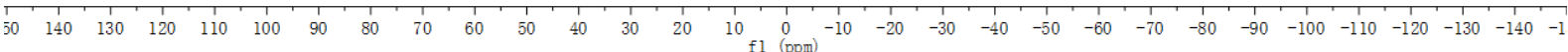

3ae ${ }^{19} \mathrm{~F}\left\{{ }^{1} \mathrm{H}\right\}$ NMR $\left(\mathrm{CDCl}_{3}, 471 \mathrm{MHz}\right)$

in

i<smiles>CCCCOc1ccccc1-c1ccccc1C(=Cc1ccc(C(F)(F)F)cc1)c1ccc(C(F)(F)F)cc1</smiles>

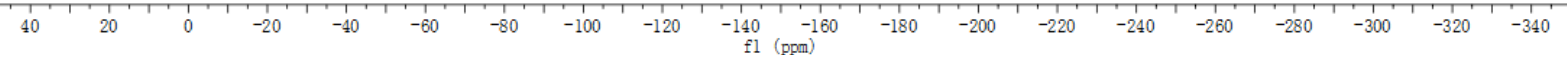

S - 93 


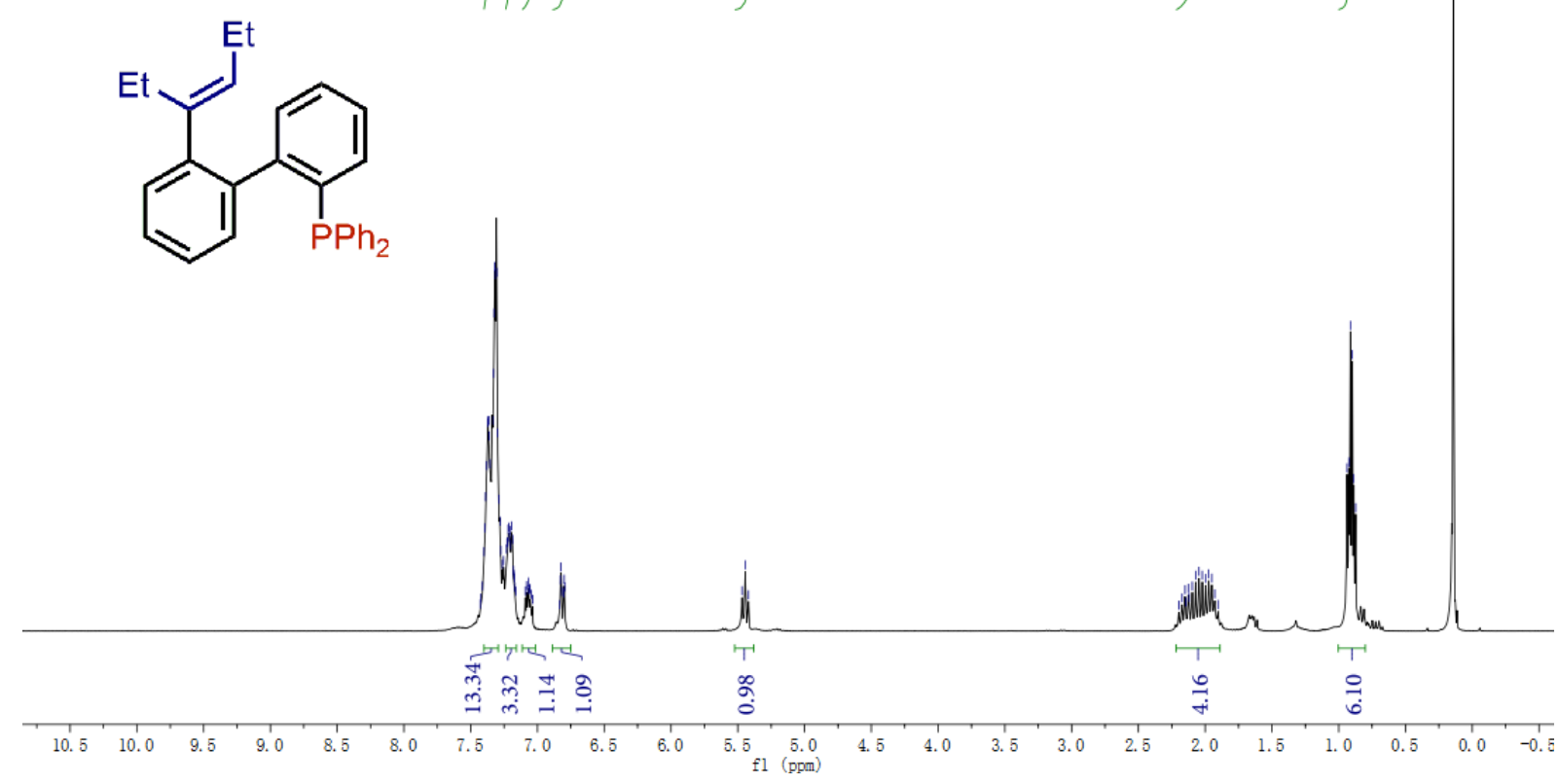

3bf $\mid{ }^{13} \mathrm{C}\left\{{ }^{1} \mathrm{H}\right\}$ NMR $\left(\mathrm{CDCl}_{3}, 101 \mathrm{MHz}\right)$

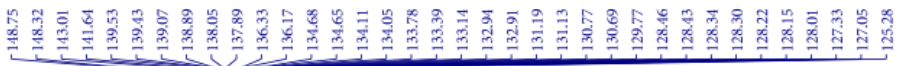<smiles>C/C=C(\CC)c1ccccc1-c1ccccc1</smiles>

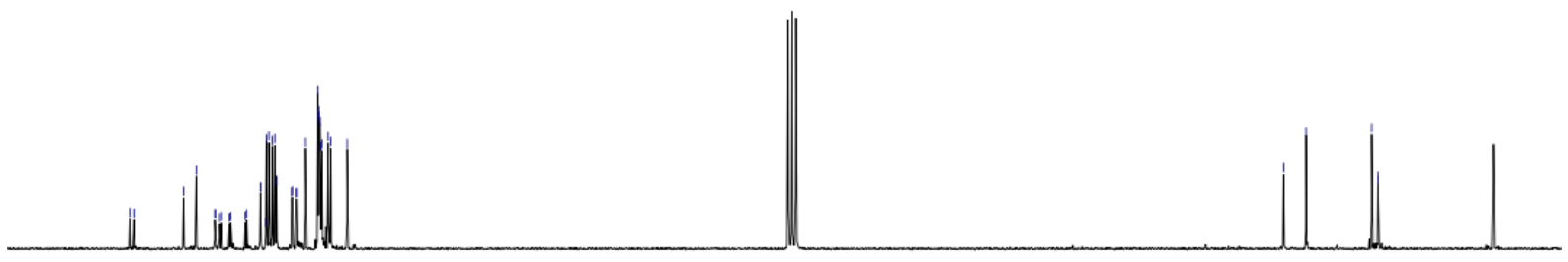

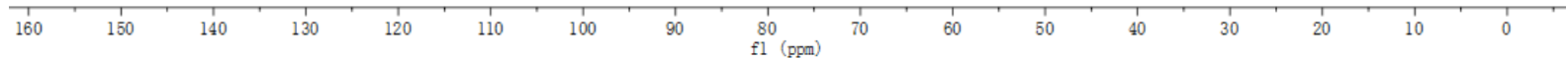


3bf $\mid{ }^{31} \mathrm{P}\left\{{ }^{1} \mathrm{H}\right\}$ NMR $\left(\mathrm{CDCl}_{3}, 162 \mathrm{MHz}\right)$

$\stackrel{?}{\stackrel{+}{i}}$<smiles>C/C=C(\CC)c1ccccc1-c1ccccc1P</smiles>

\begin{tabular}{rllllllllllllllllllllllllllllllllllll}
\hline 140 & 130 & 120 & 110 & 100 & 90 & 80 & 70 & 60 & 50 & 40 & 30 & 20 & 10 & 0 & -10 & -20 & -30 & -40 & -50 & -60 & -70 & -80 & -90 & -100 & -110 & -120 & -130 & -140
\end{tabular} 
3hf | ${ }^{1} \mathrm{H} \mathrm{NMR}\left(\mathrm{CDCl}_{3}, 400 \mathrm{MHz}\right)$

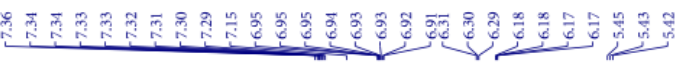

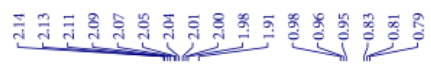

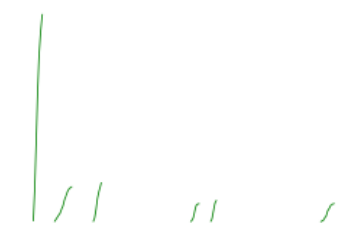<smiles>CC=C(CC)c1ccccc1-n1cccc1-c1ccccc1</smiles>

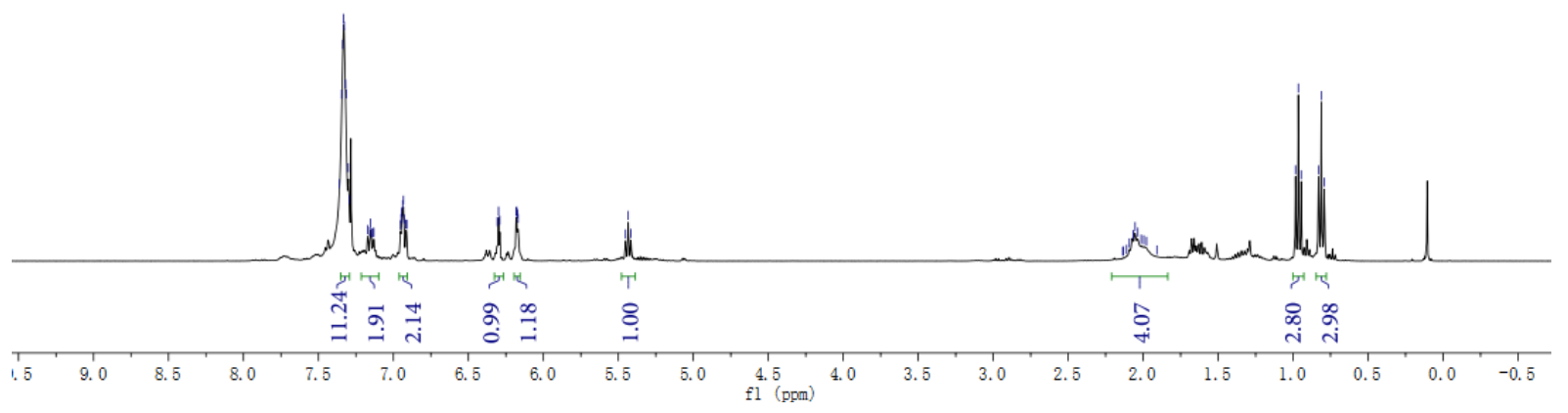

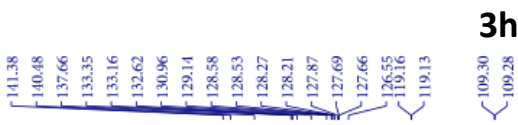

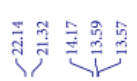<smiles>CC/C=C(\CC)c1ccccc1-n1cccc1-c1ccccc1</smiles>

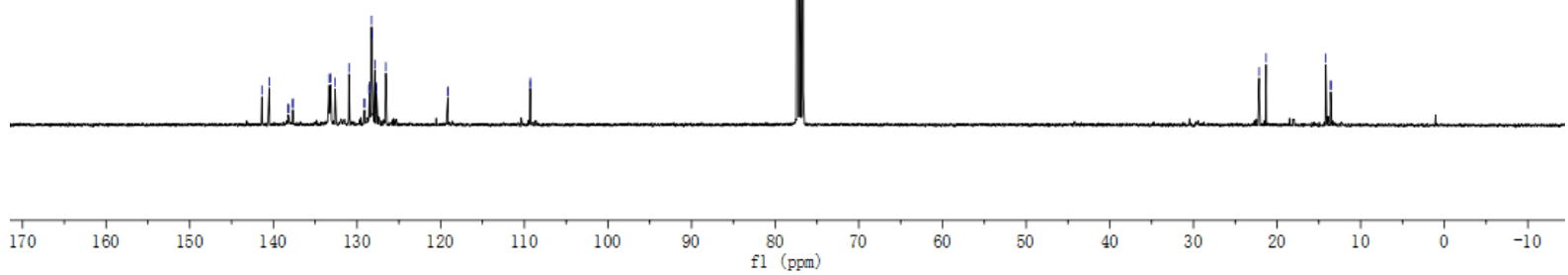

S - 96 
3hf $\mid{ }^{31} \mathrm{P}\left\{{ }^{1} \mathrm{H}\right\}$ NMR $\left(\mathrm{CDCl}_{3}, 162 \mathrm{MHz}\right)$<smiles>C/C=C(/CC)c1ccccc1-n1cccc1P</smiles>

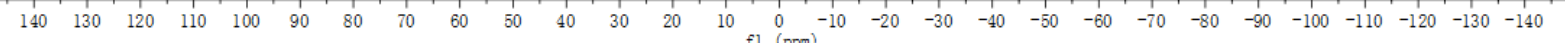




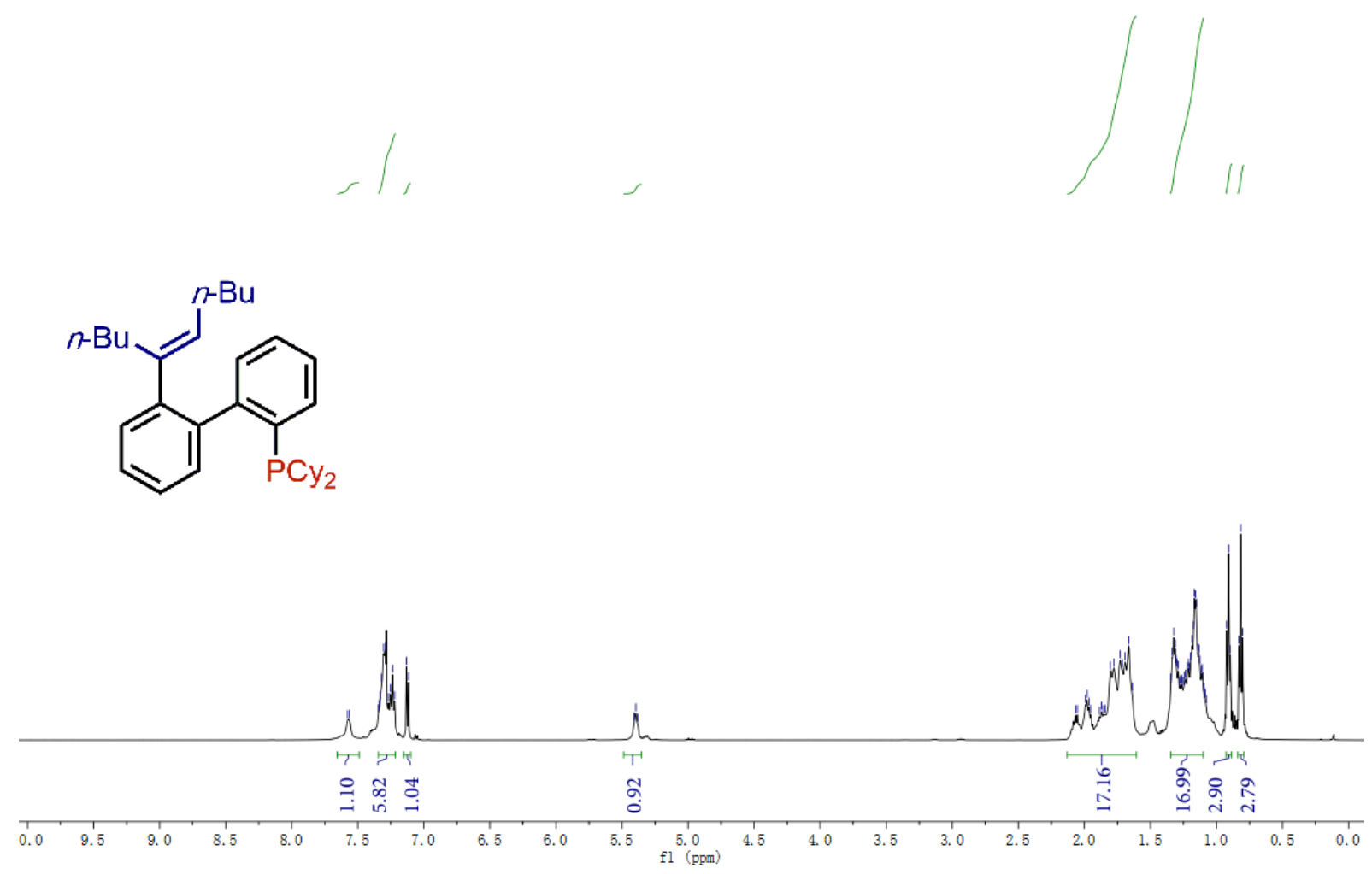

3ag $\mid{ }^{13} \mathrm{C}\left\{{ }^{1} \mathrm{H}\right\} \mathrm{NMR}\left(\mathrm{CDCl}_{3}, 101 \mathrm{MHz}\right)$

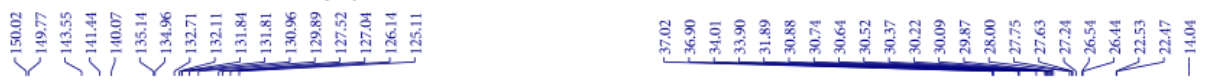<smiles>CCCC=C(C(=CCCCC)CCCC)c1ccccc1-c1ccccc1PC(C)(C)C</smiles>

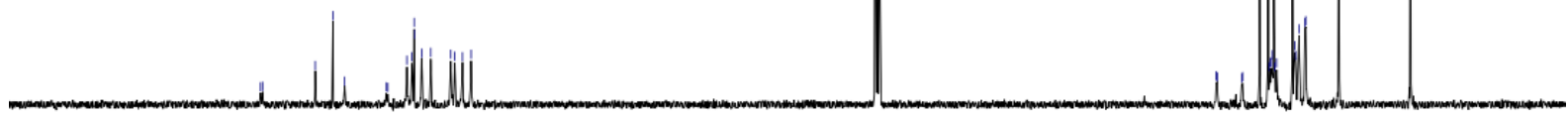

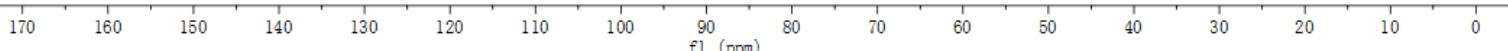


3ag $\left.\right|^{31} \mathrm{P}\left\{{ }^{1} \mathrm{H}\right\} \mathrm{NMR}\left(\mathrm{CDCl}_{3}, 162 \mathrm{MHz}\right)$

$\stackrel{+}{\vec{H}}$

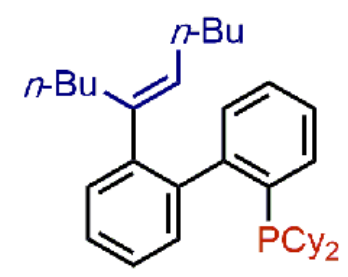

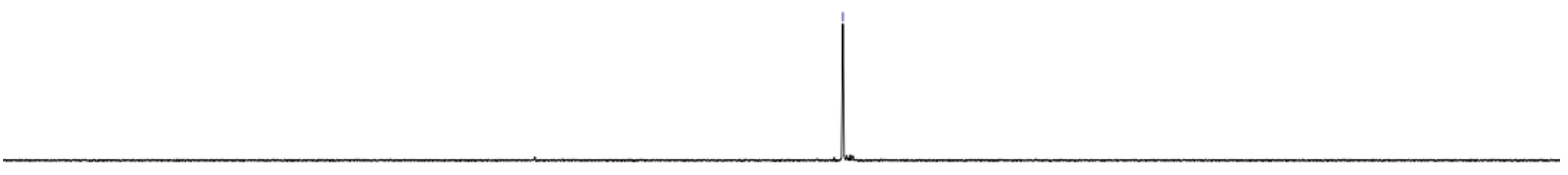

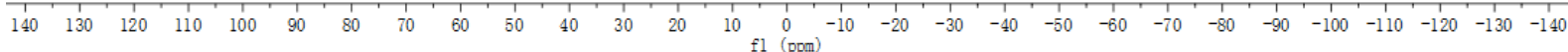




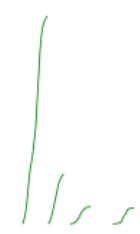

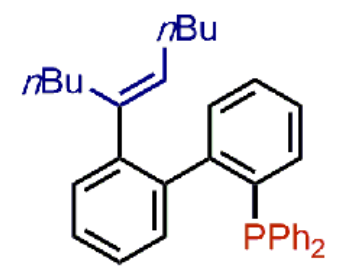

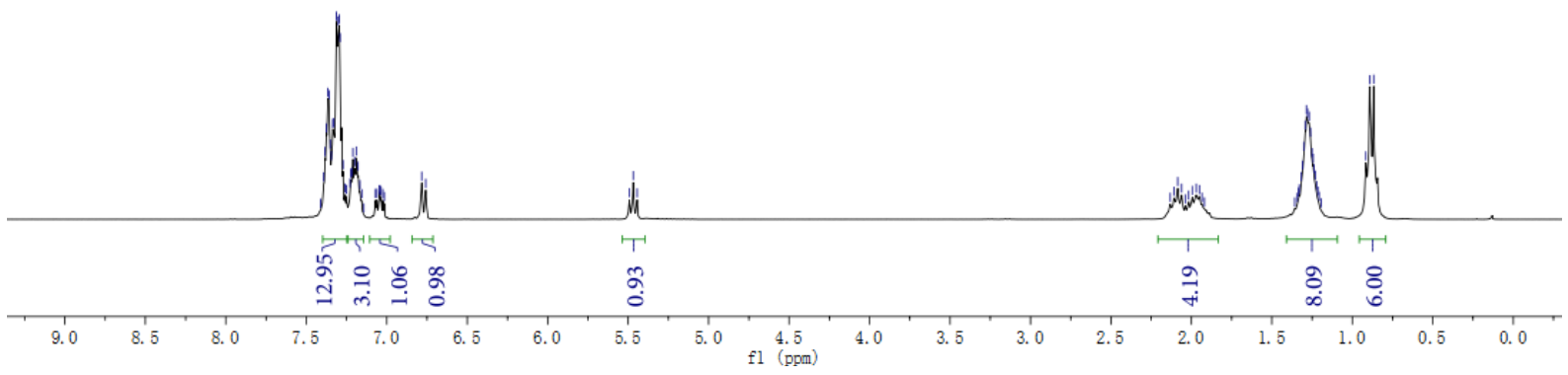

3bg $\mid{ }^{13} \mathrm{C}\left\{{ }^{1} \mathrm{H}\right\} \mathrm{NMR}\left(\mathrm{CDCl}_{3}, 101 \mathrm{MHz}\right)$

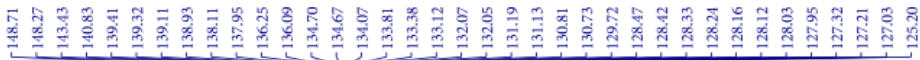

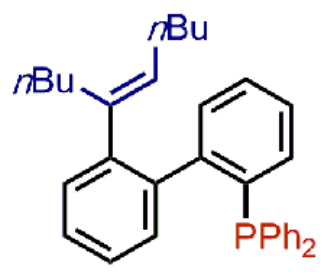

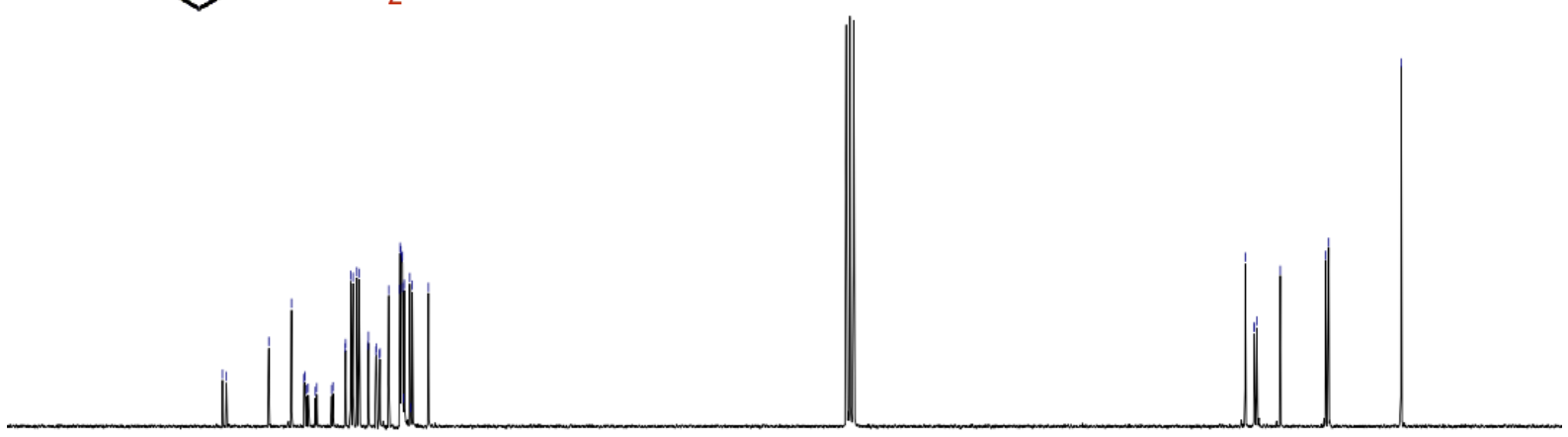

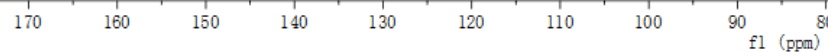


3bg $\mid{ }^{31} \mathrm{P}\left\{{ }^{1} \mathrm{H}\right\} \mathrm{NMR}\left(\mathrm{CDCl}_{3}, 162 \mathrm{MHz}\right)$

$\stackrel{\infty}{\stackrel{+}{i}}$<smiles>CCCC=C(CCCC)C(=CCCCC)c1ccccc1-c1ccccc1</smiles>

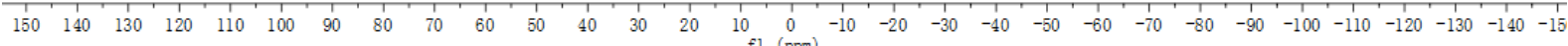


<smiles>C(=C(c1ccccc1)c1cccc(C(=Cc2ccccc2)c2ccccc2)c1-c1ccccc1)c1ccccc1</smiles>
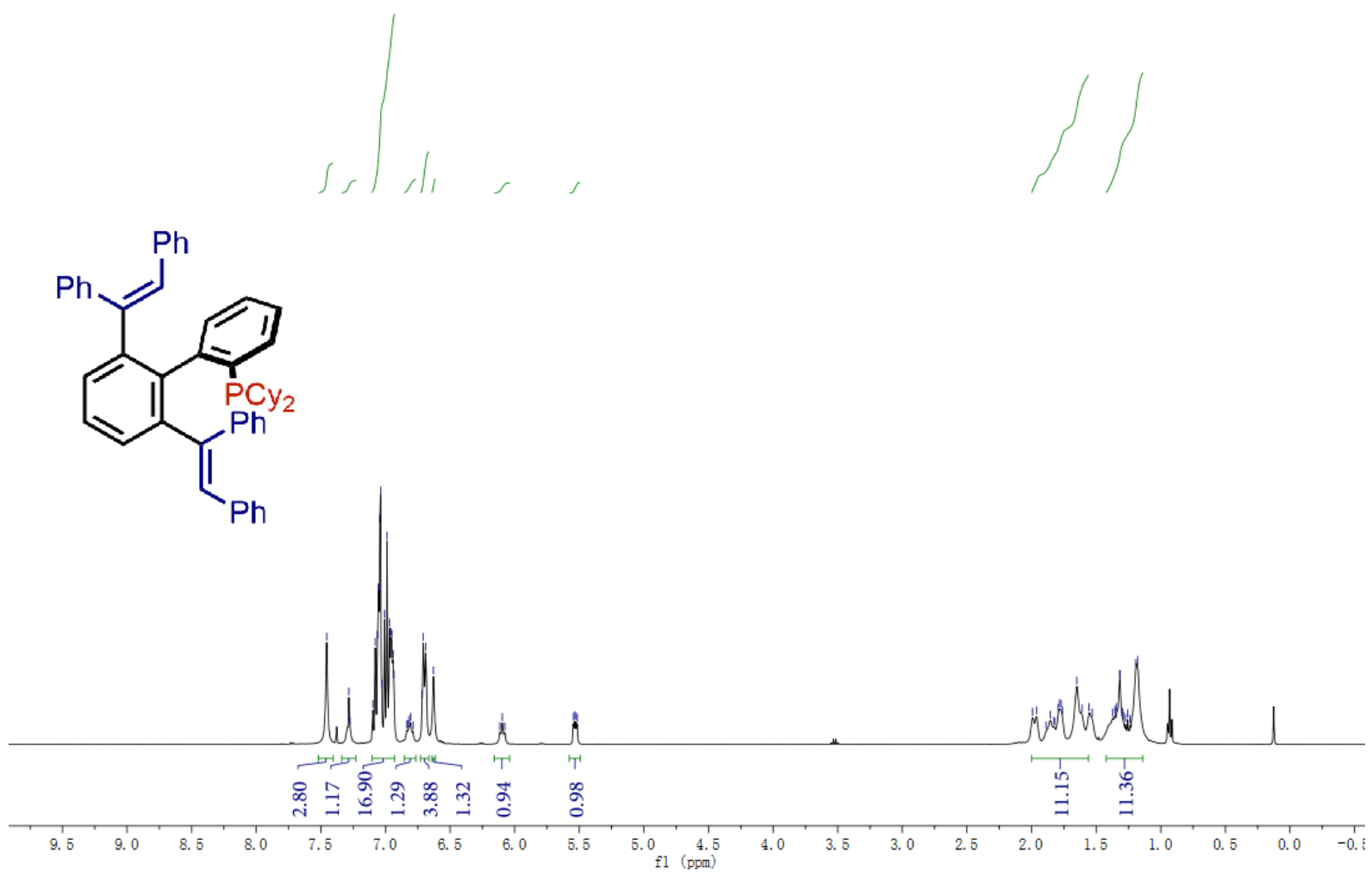

4aa $\mid{ }^{13} \mathrm{C}\left\{{ }^{1} \mathrm{H}\right\}$ NMR $\left(\mathrm{CDCl}_{3}, 101 \mathrm{MHz}\right)$

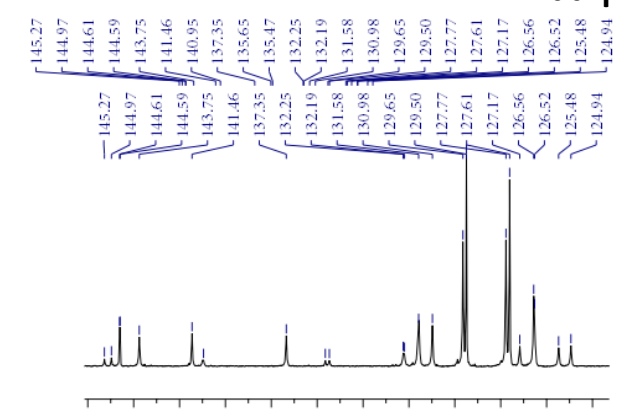

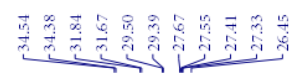
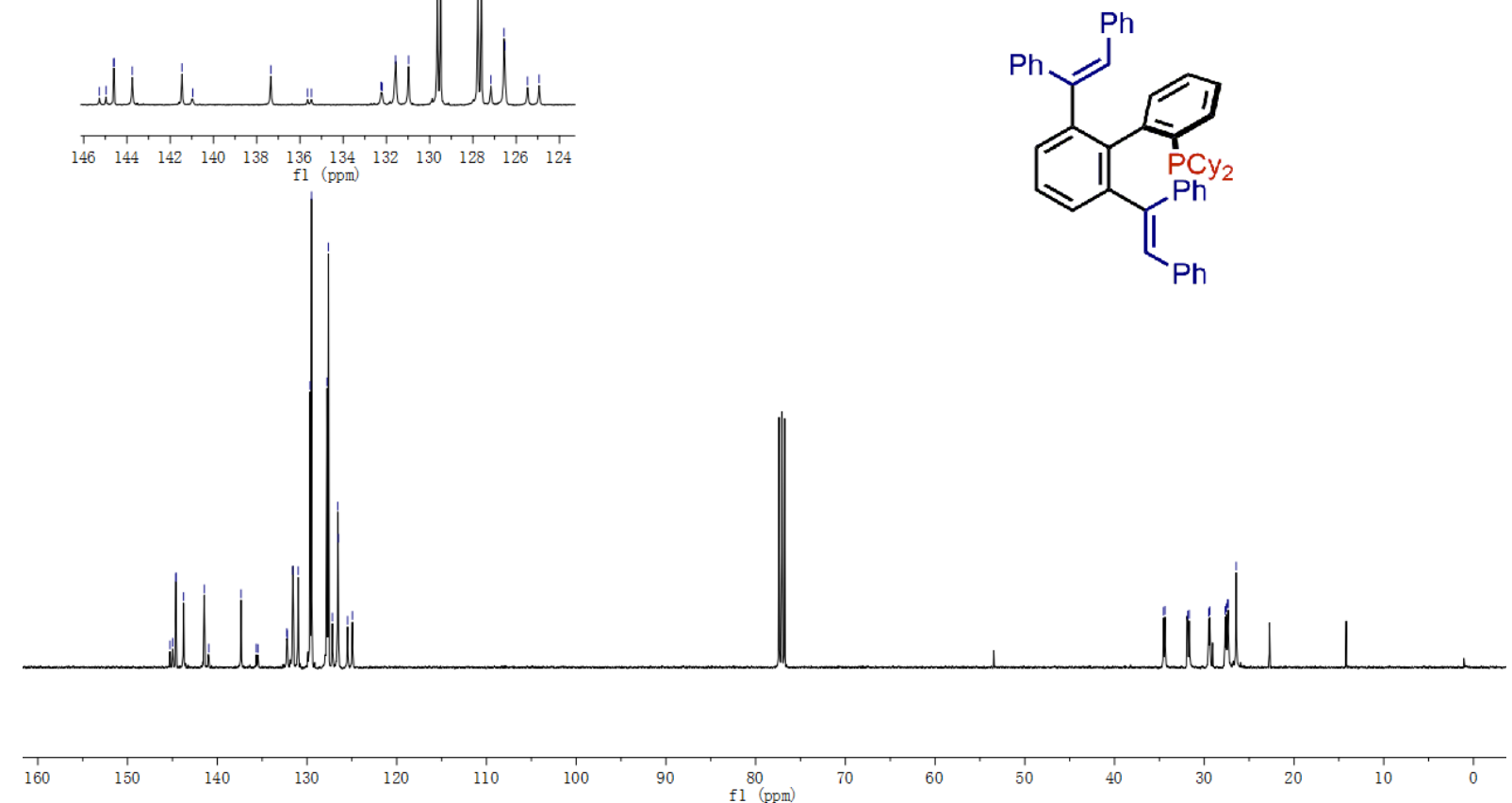

$$
\text { S - } 102
$$


4aa | ${ }^{31} \mathrm{P}\left\{{ }^{1} \mathrm{H}\right\} \mathrm{NMR}\left(\mathrm{CDCl}_{3}, 162 \mathrm{MHz}\right)$

$\hat{i}$<smiles>C(=C(c1ccccc1)c1cccc(-c2ccccc2)c1C(=Cc1ccccc1)c1ccccc1)c1ccccc1</smiles>

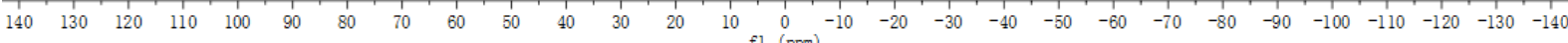



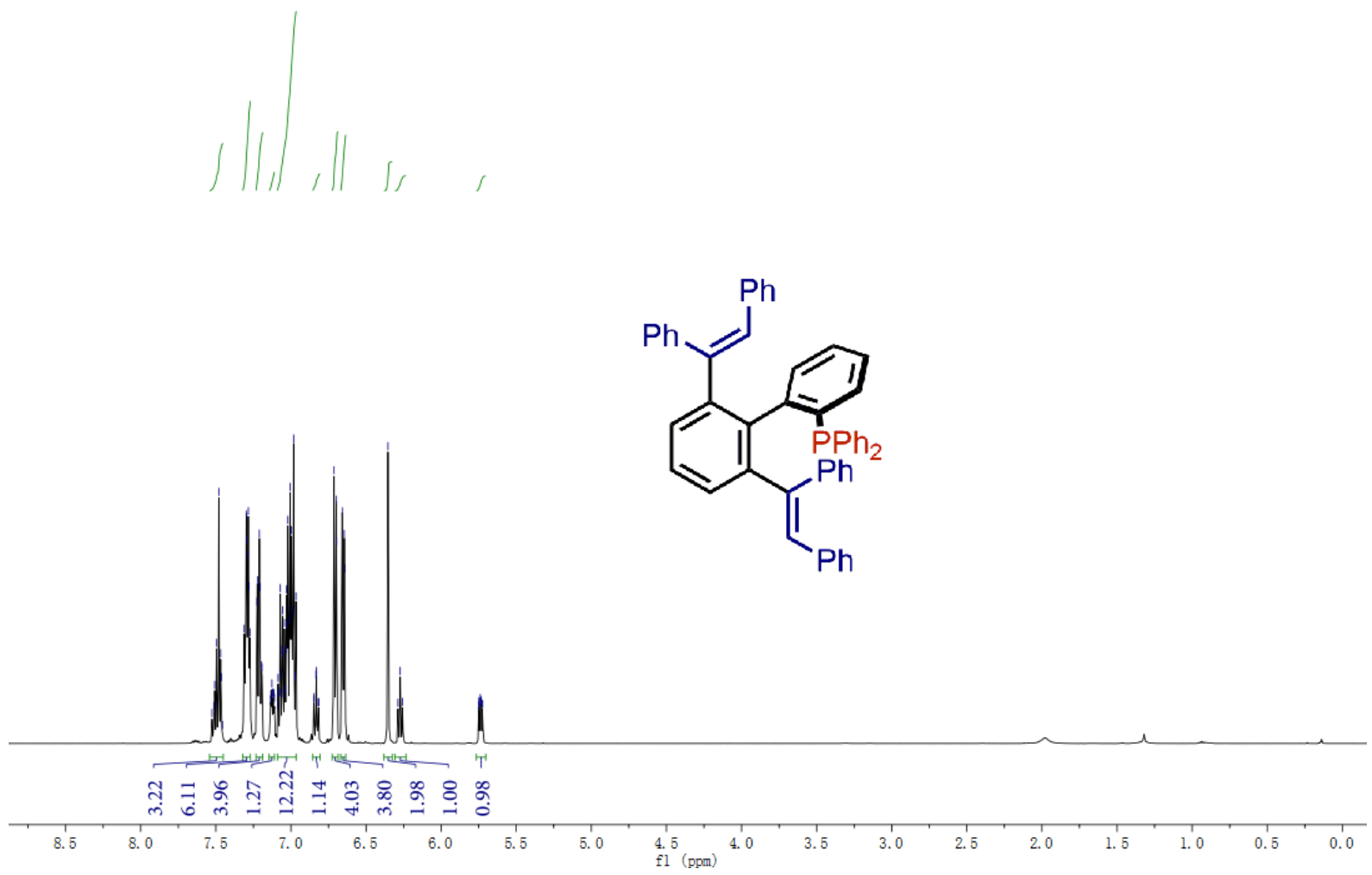

4ba $\mid{ }^{13} \mathrm{C}\left\{{ }^{1} \mathrm{H}\right\}$ NMR $\left(\mathrm{CDCl}_{3}, 101 \mathrm{MHz}\right)$

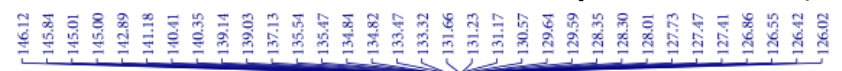

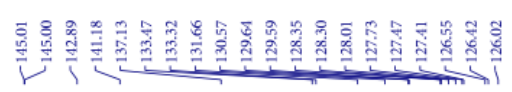
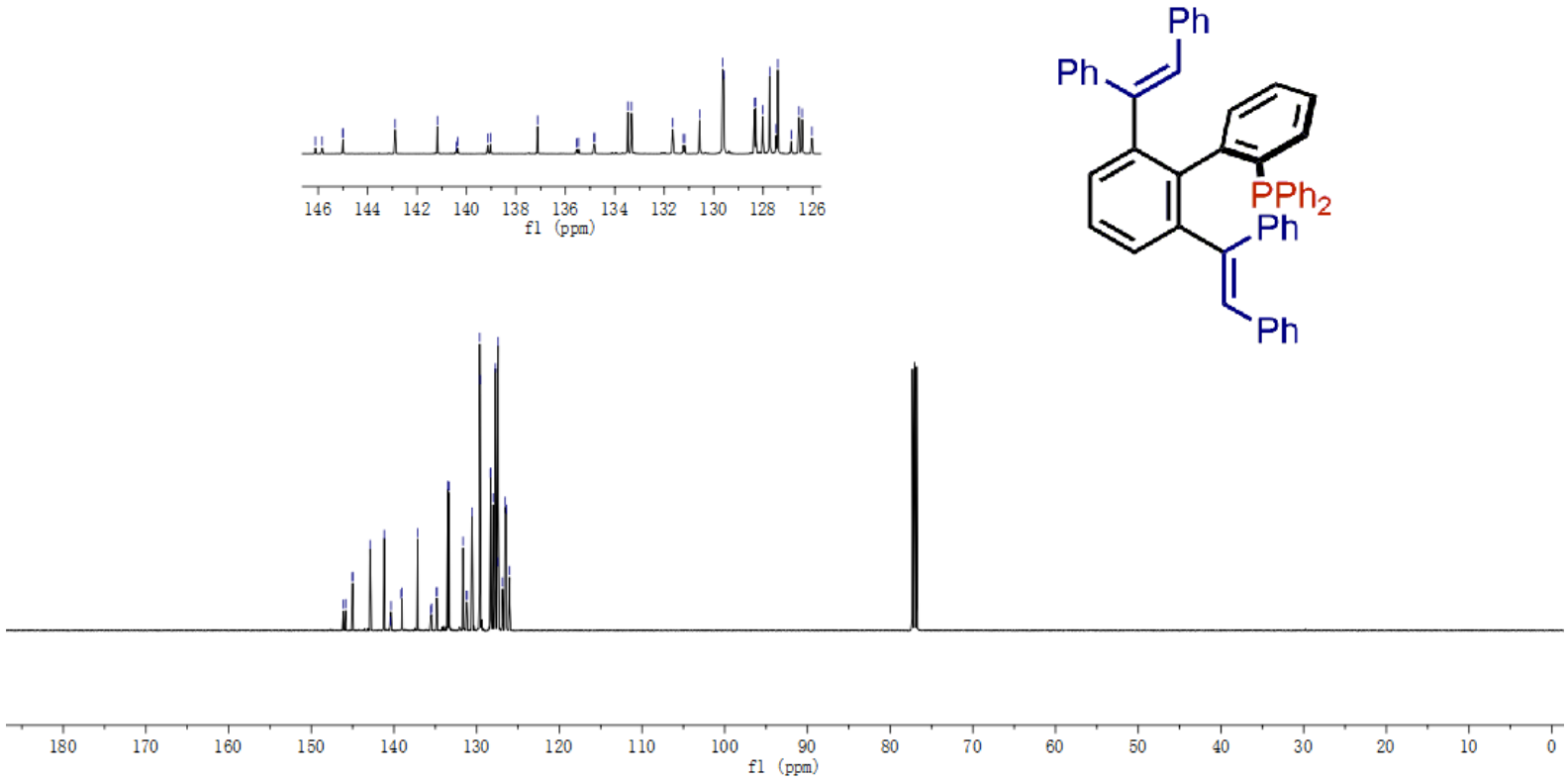
4ba | ${ }^{31} \mathrm{P}\left\{{ }^{1} \mathrm{H}\right\} \mathrm{NMR}\left(\mathrm{CDCl}_{3}, 162 \mathrm{MHz}\right)$

i<smiles>C(=C(c1ccccc1)c1ccccc1C(=Cc1ccccc1)c1ccccc1)c1ccccc1</smiles>

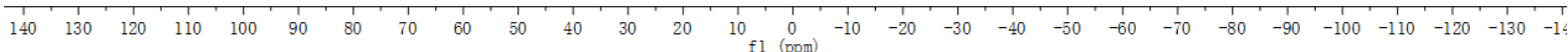


<smiles>O=P(c1ccccc1)(c1ccccc1)c1cccc(C(=Cc2ccccc2)c2ccccc2)c1C(=Cc1ccccc1)c1ccccc1</smiles>

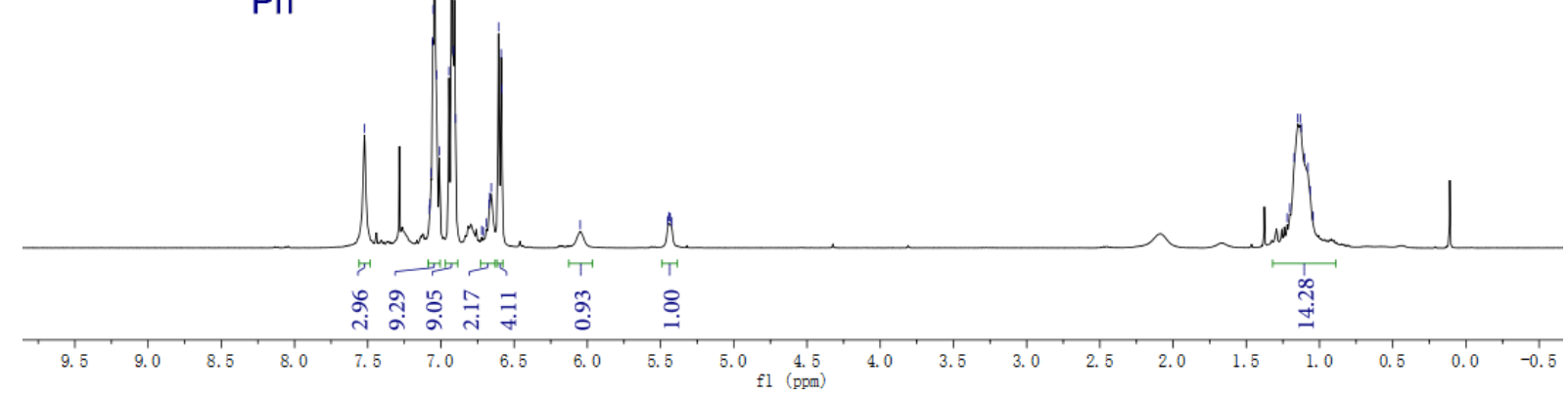

4ca $\mid{ }^{13} \mathrm{C}\left\{{ }^{1} \mathrm{H}\right\}$ NMR $\left(\mathrm{CDCl}_{3}, 101 \mathrm{MHz}\right)$
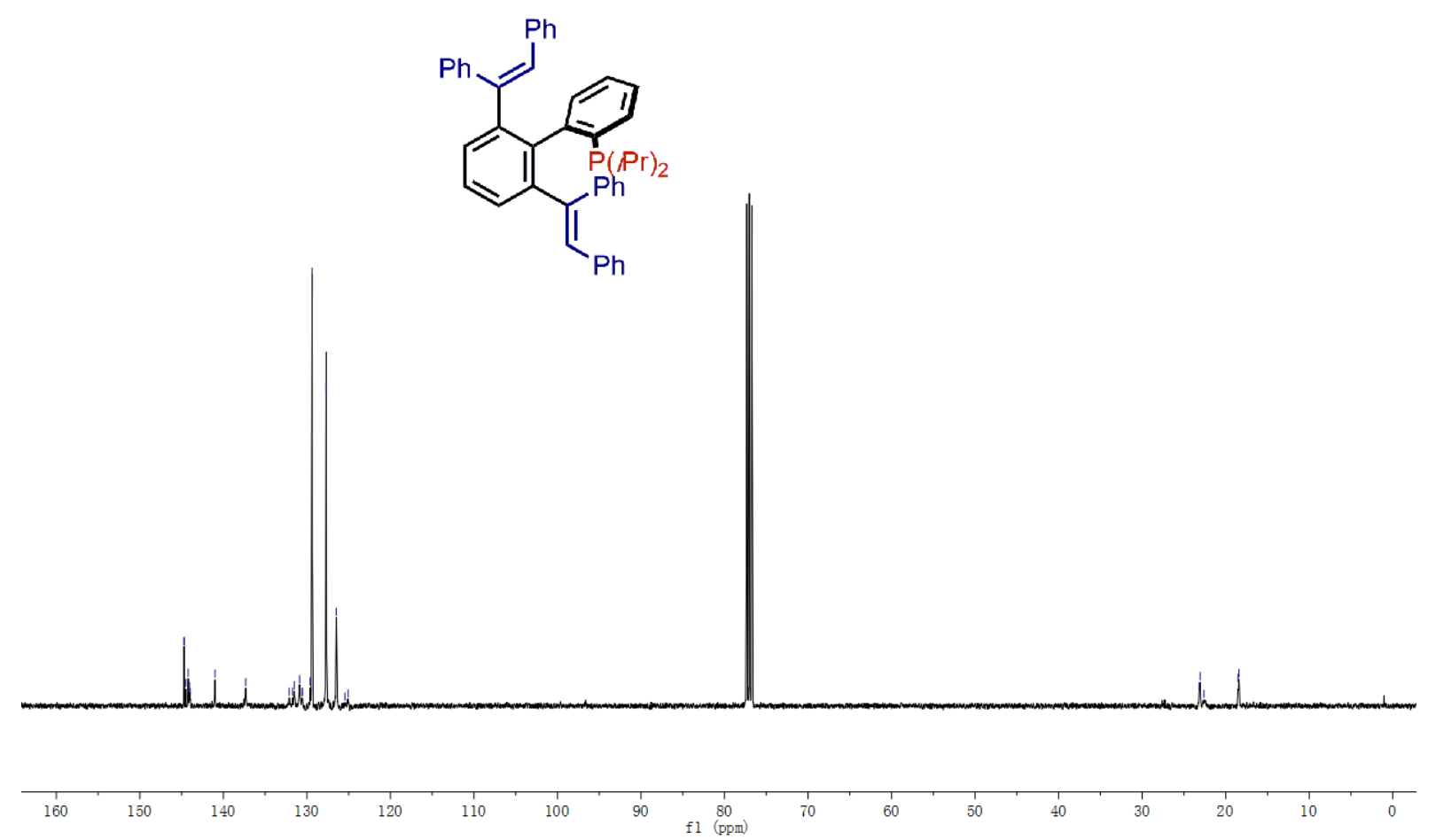
4ca | ${ }^{31} \mathrm{P}\left\{{ }^{1} \mathrm{H}\right\} \mathrm{NMR}\left(\mathrm{CDCl}_{3}, 162 \mathrm{MHz}\right)$

$\vec{i}$<smiles>Pc1ccccc1/C=C(\c1ccccc1)c1ccccc1/C(=C/c1ccccc1)c1ccccc1</smiles>

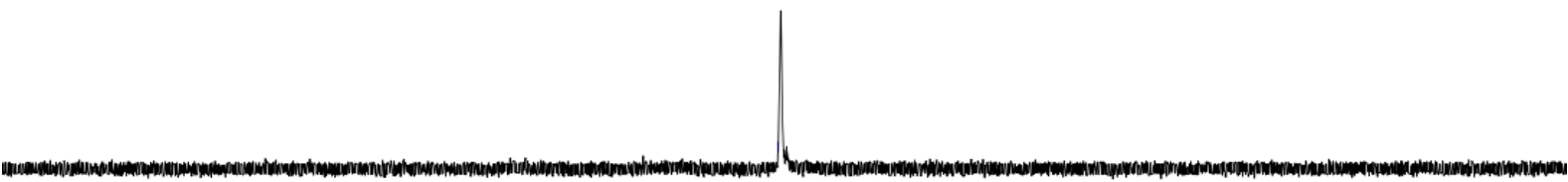

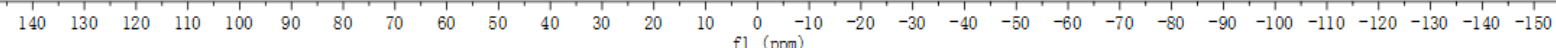


4ga | ${ }^{1} \mathrm{H} \mathrm{NMR}\left(\mathrm{CDCl}_{3}, 400 \mathrm{MHz}\right)$

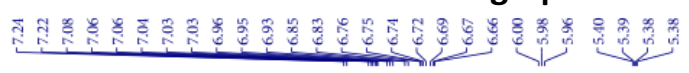
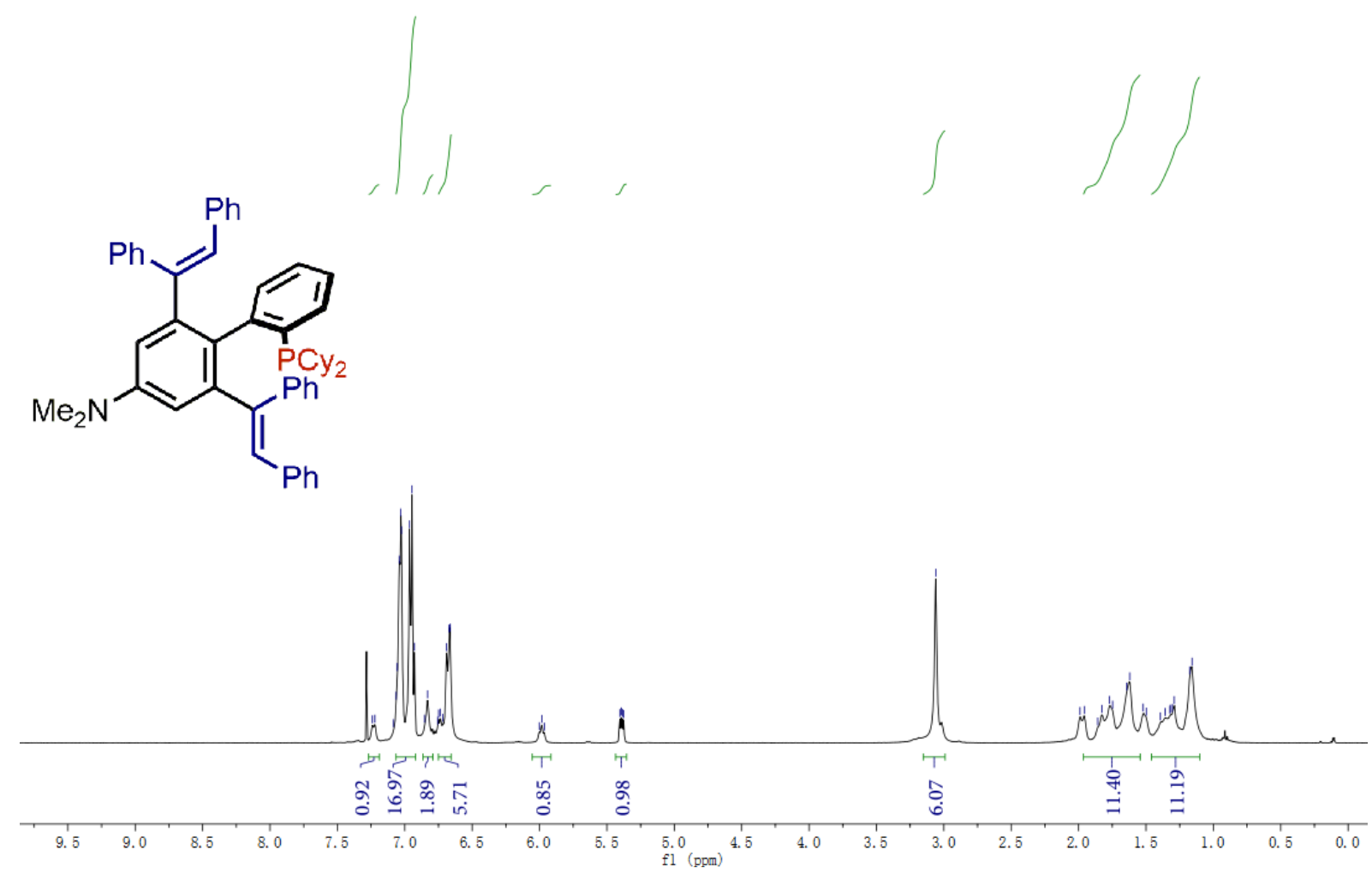

4ga $\mid{ }^{13} \mathrm{C}\left\{{ }^{1} \mathrm{H}\right\} \operatorname{NMR}\left(\mathrm{CDCl}_{3}, 101 \mathrm{MHz}\right)$

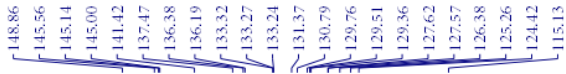<smiles>CN(C)c1cc(/C(=C/c2ccccc2)c2ccccc2)c(-c2ccccc2)c(-c2ccccc2)c1</smiles>

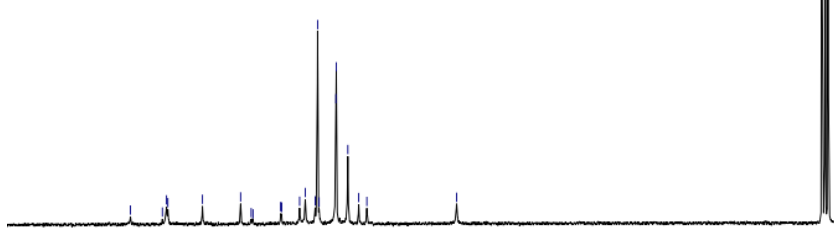

i i i i

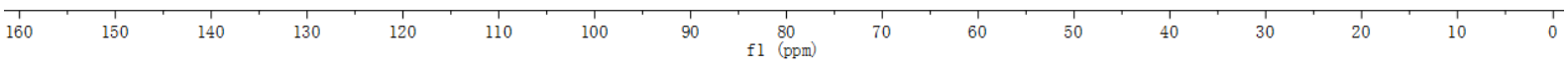

$S-108$ 
4ga | ${ }^{31} \mathrm{P}\left\{{ }^{1} \mathrm{H}\right\} \mathrm{NMR}\left(\mathrm{CDCl}_{3}, 162 \mathrm{MHz}\right)$<smiles>CN(C)c1cc(/C=C/c2ccccc2)c(-c2ccccc2)c(/C(=C/c2ccccc2)c2ccccc2)c1</smiles>

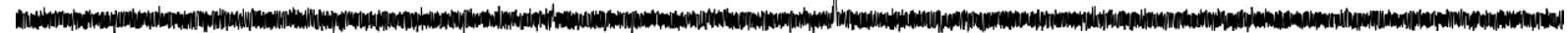
\begin{tabular}{llllllllllllllllllllllllllllllllll}
\hline 40 & 130 & 120 & 110 & 100 & 90 & 80 & 70 & 60 & 50 & 40 & 30 & 20 & 10 & 0 & -10 & -20 & -30 & -40 & -50 & -60 & -70 & -80 & -90 & -100 & -110 & -120 & -130 & -1
\end{tabular} 

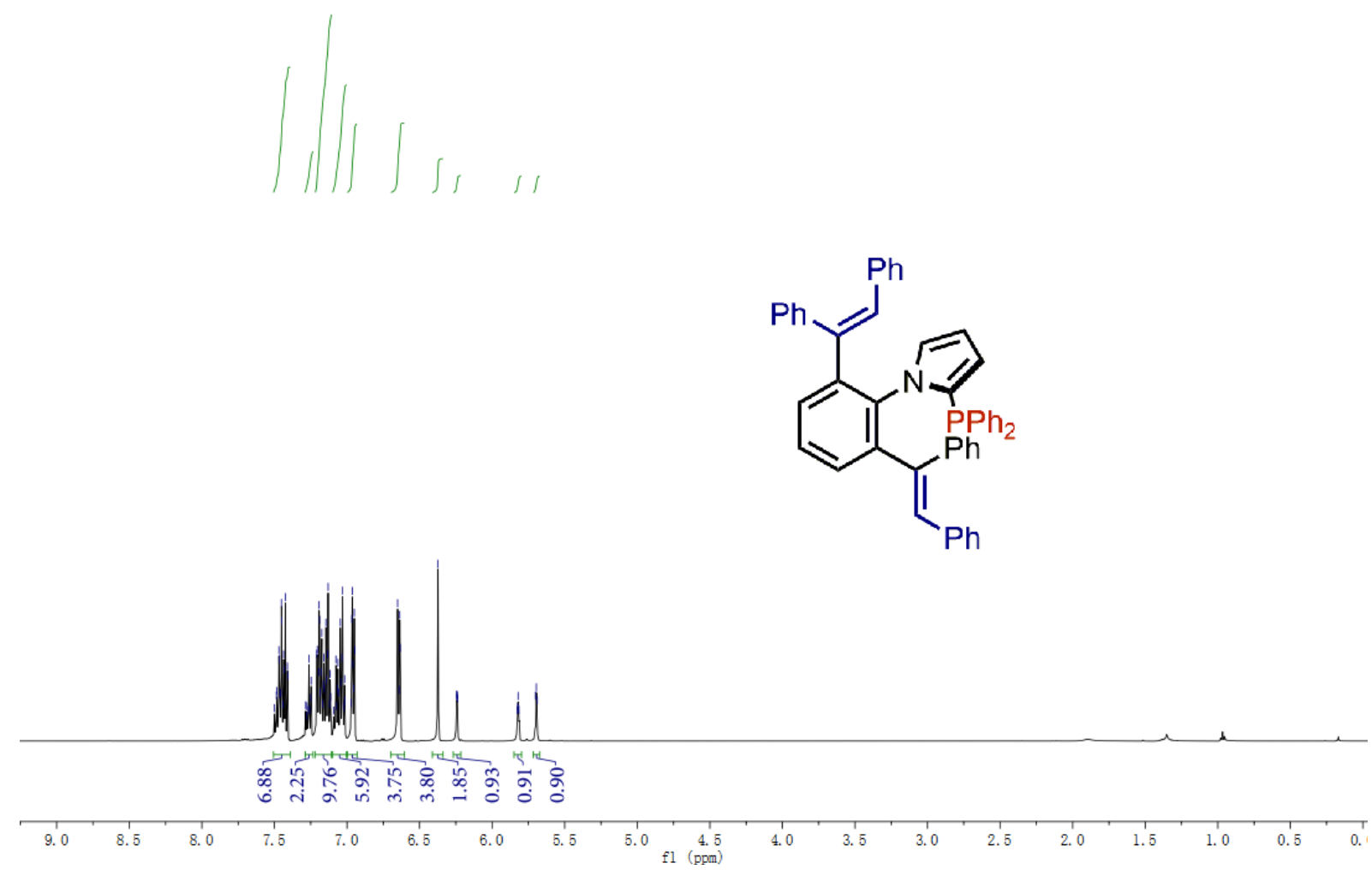

4ha $\mid{ }^{13} \mathrm{C}\left\{{ }^{1} \mathrm{H}\right\}$ NMR $\left(\mathrm{CDCl}_{3}, 101 \mathrm{MHz}\right)$

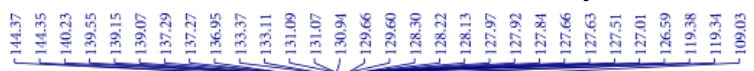

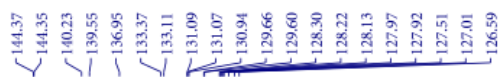
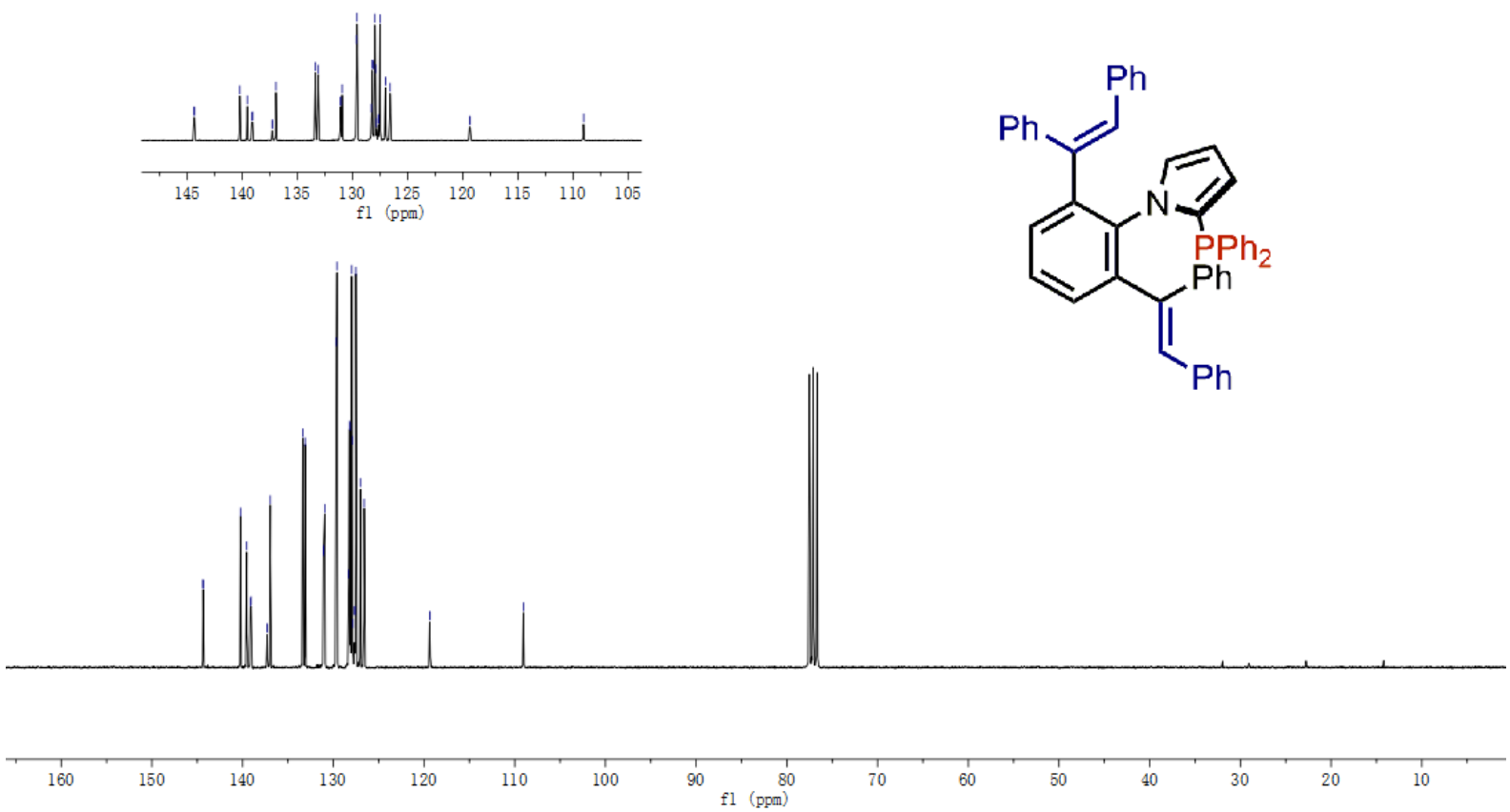

$S-110$ 
4ha | ${ }^{31} \mathrm{P}\left\{{ }^{1} \mathrm{H}\right\} \mathrm{NMR}\left(\mathrm{CDCl}_{3}, 162 \mathrm{MHz}\right)$

iे<smiles>C(=C(c1ccccc1)c1cccc(-c2ccccc2)c1-c1ccccc1)c1ccccc1</smiles>

$\begin{array}{lllllllllllllllllllllllllllllll}140 & 130 & 120 & 110 & 100 & 90 & 80 & 70 & 60 & 50 & 40 & 30 & 20 & 10 & 0 & -10 & -20 & -30 & -40 & -50 & -60 & -70 & -80 & -90 & -100 & -110 & -120 & -130 & -14\end{array}$ 

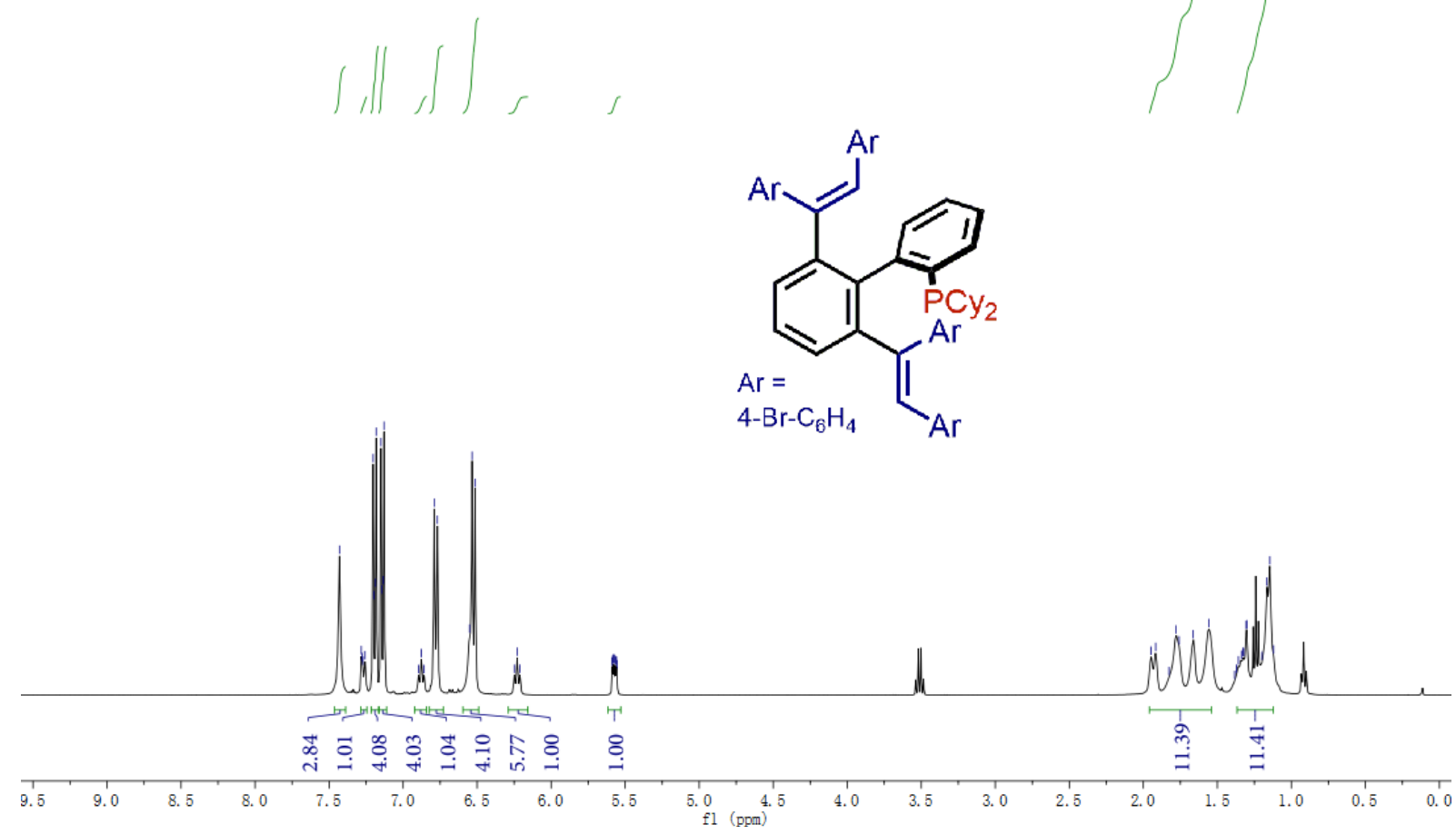

4ab $\mid{ }^{13} \mathrm{C}\left\{{ }^{1} \mathrm{H}\right\}$ NMR $\left(\mathrm{CDCl}_{3}, 101 \mathrm{MHz}\right)$

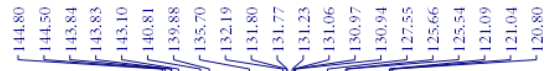
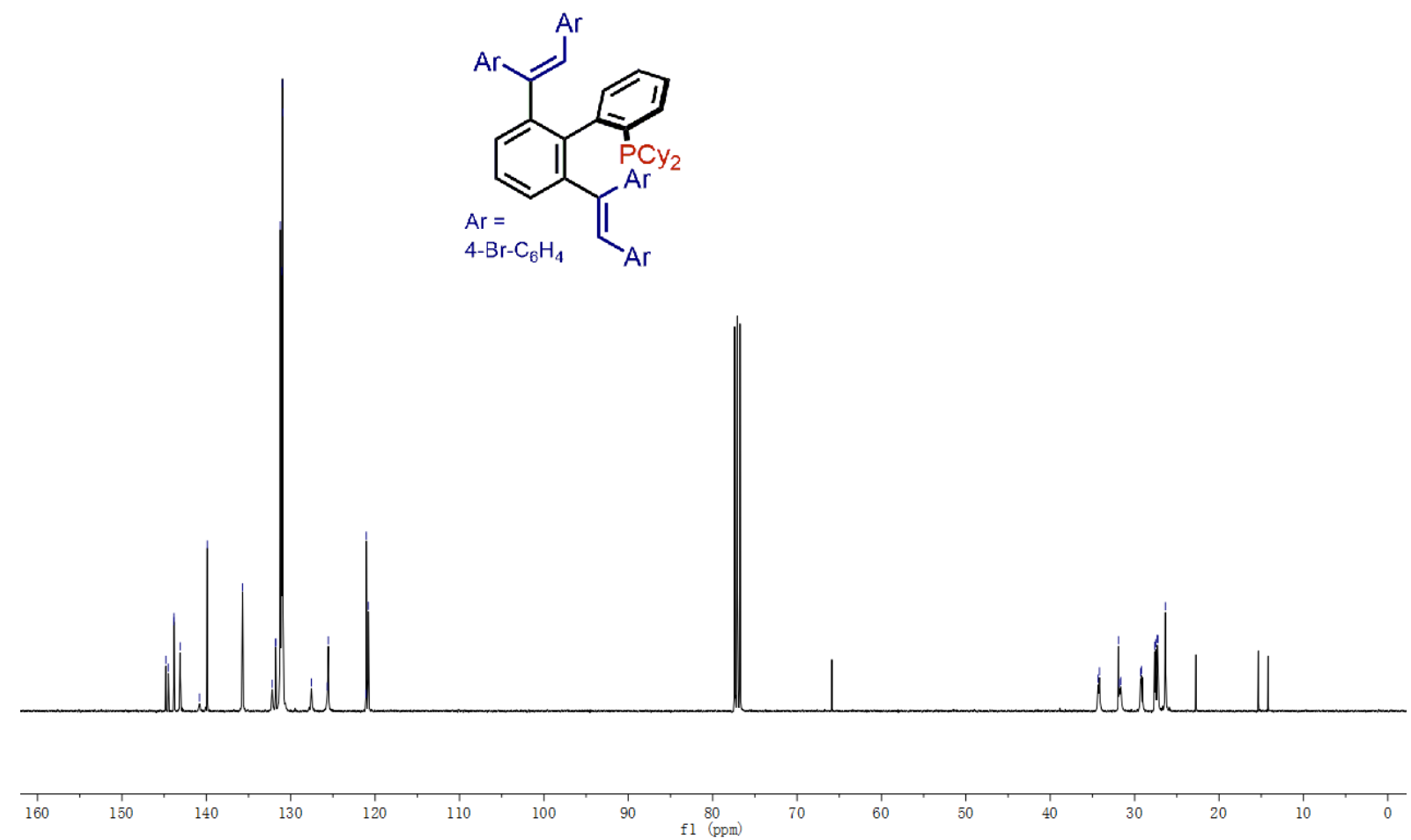
4ab ${ }^{31} \mathrm{P}\left\{{ }^{1} \mathrm{H}\right\} \mathrm{NMR}\left(\mathrm{CDCl}_{3}, 162 \mathrm{MHz}\right)$

$\stackrel{m}{i}$<smiles>Br/C=C(\Br)c1cccc(/C(Br)=C/Br)c1-c1ccccc1[Te]</smiles>

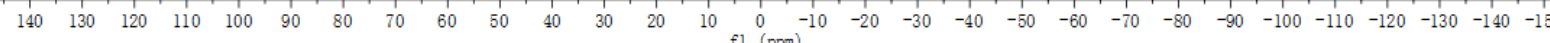




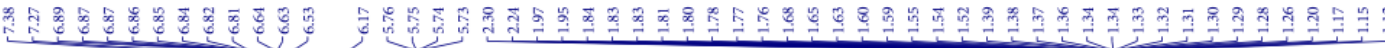
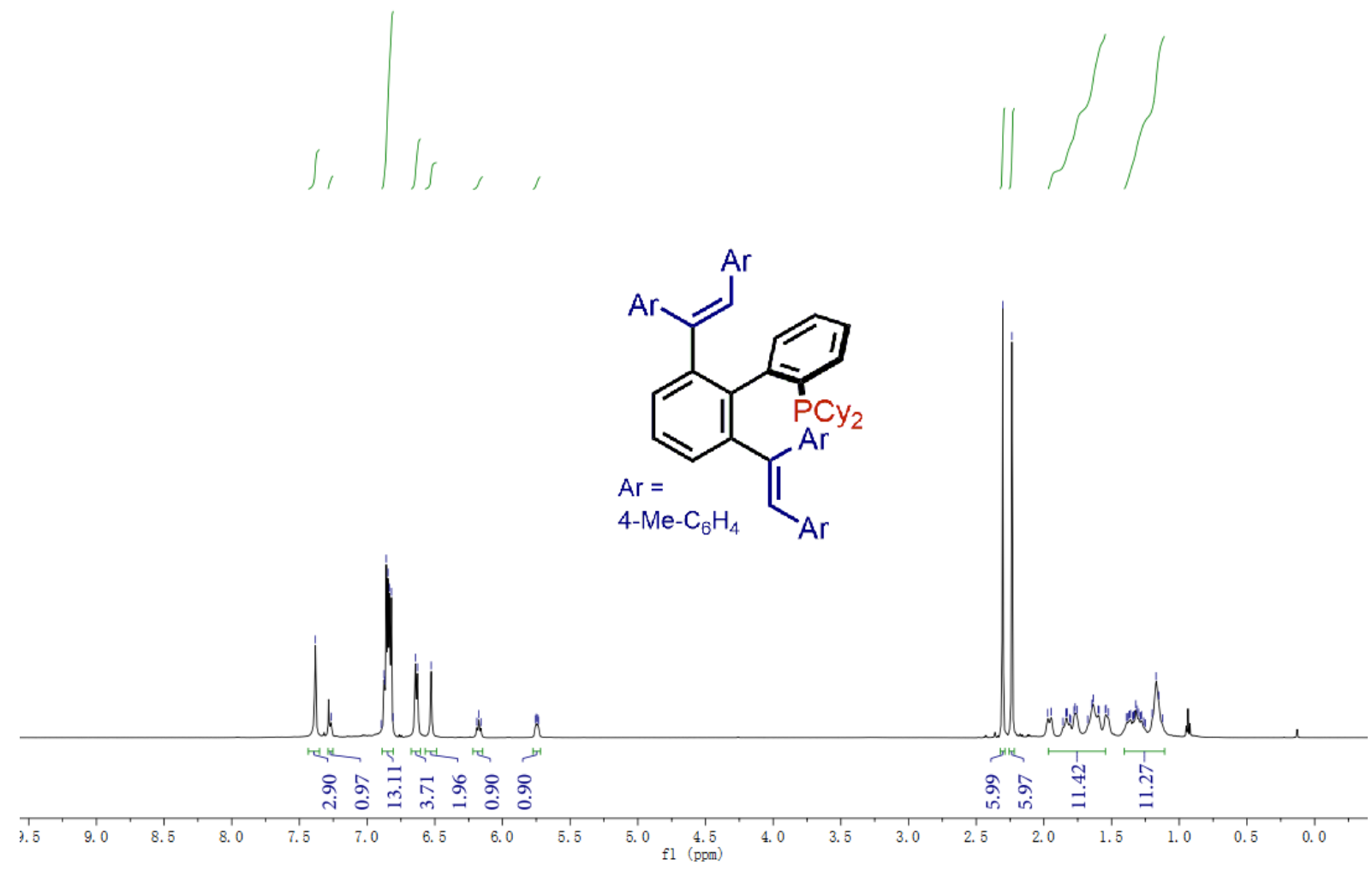

4ac $\mid{ }^{13} \mathrm{C}\left\{{ }^{1} \mathrm{H}\right\} \mathrm{NMR}\left(\mathrm{CDCl}_{3}, 101 \mathrm{MHz}\right)$

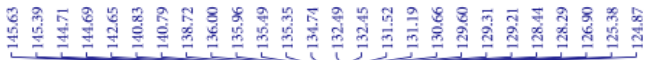
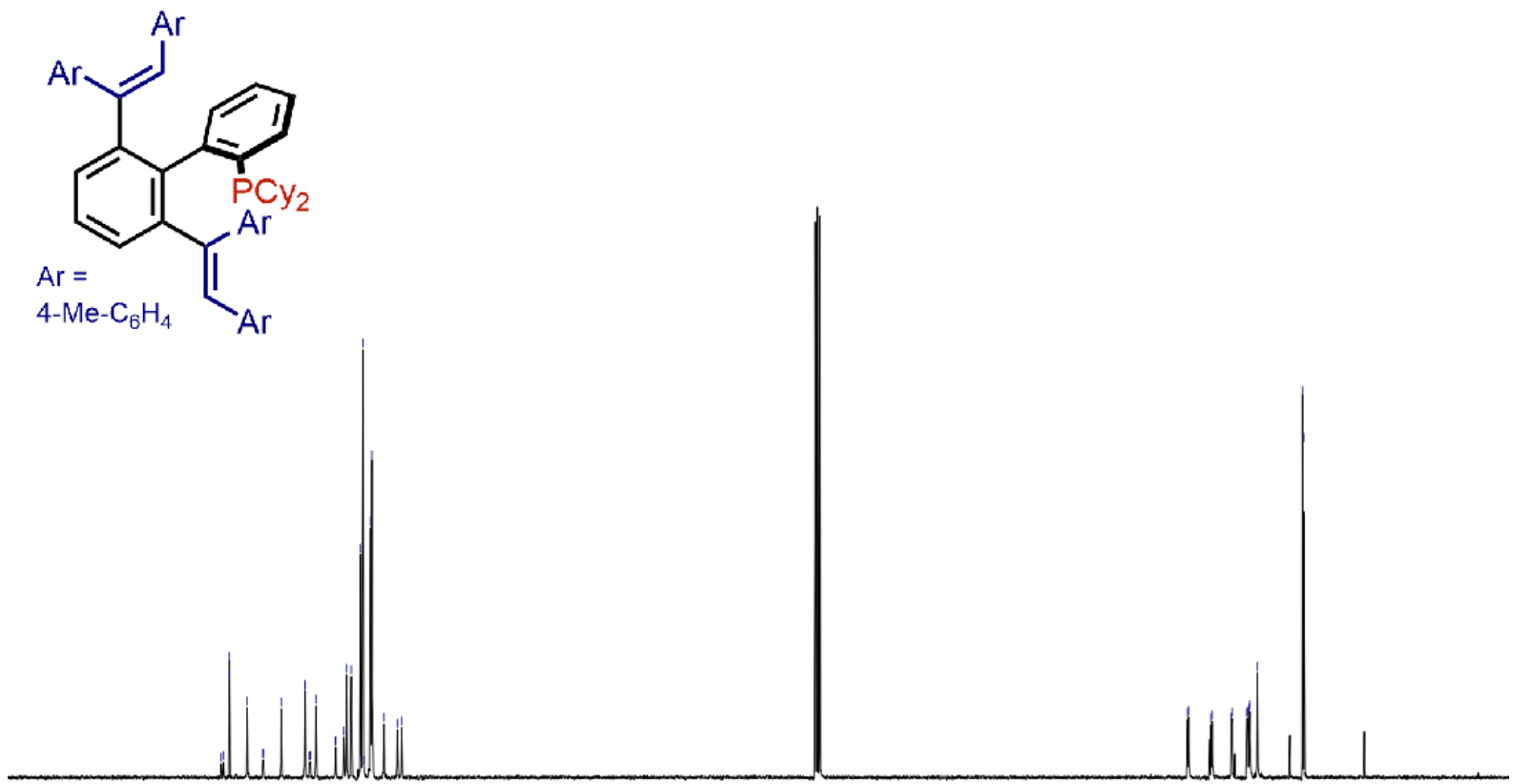

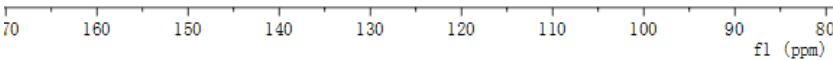


4ac ${ }^{31} \mathrm{P}\left\{{ }^{1} \mathrm{H}\right\} \mathrm{NMR}\left(\mathrm{CDCl}_{3}, 162 \mathrm{MHz}\right)$

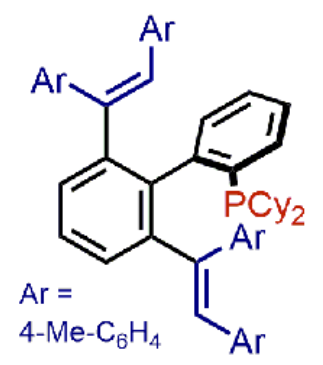

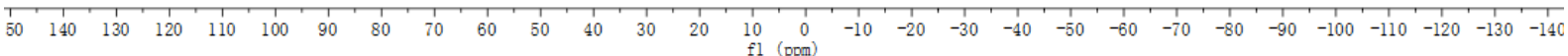




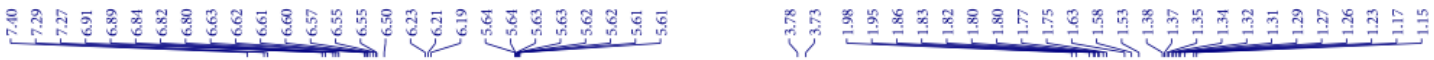
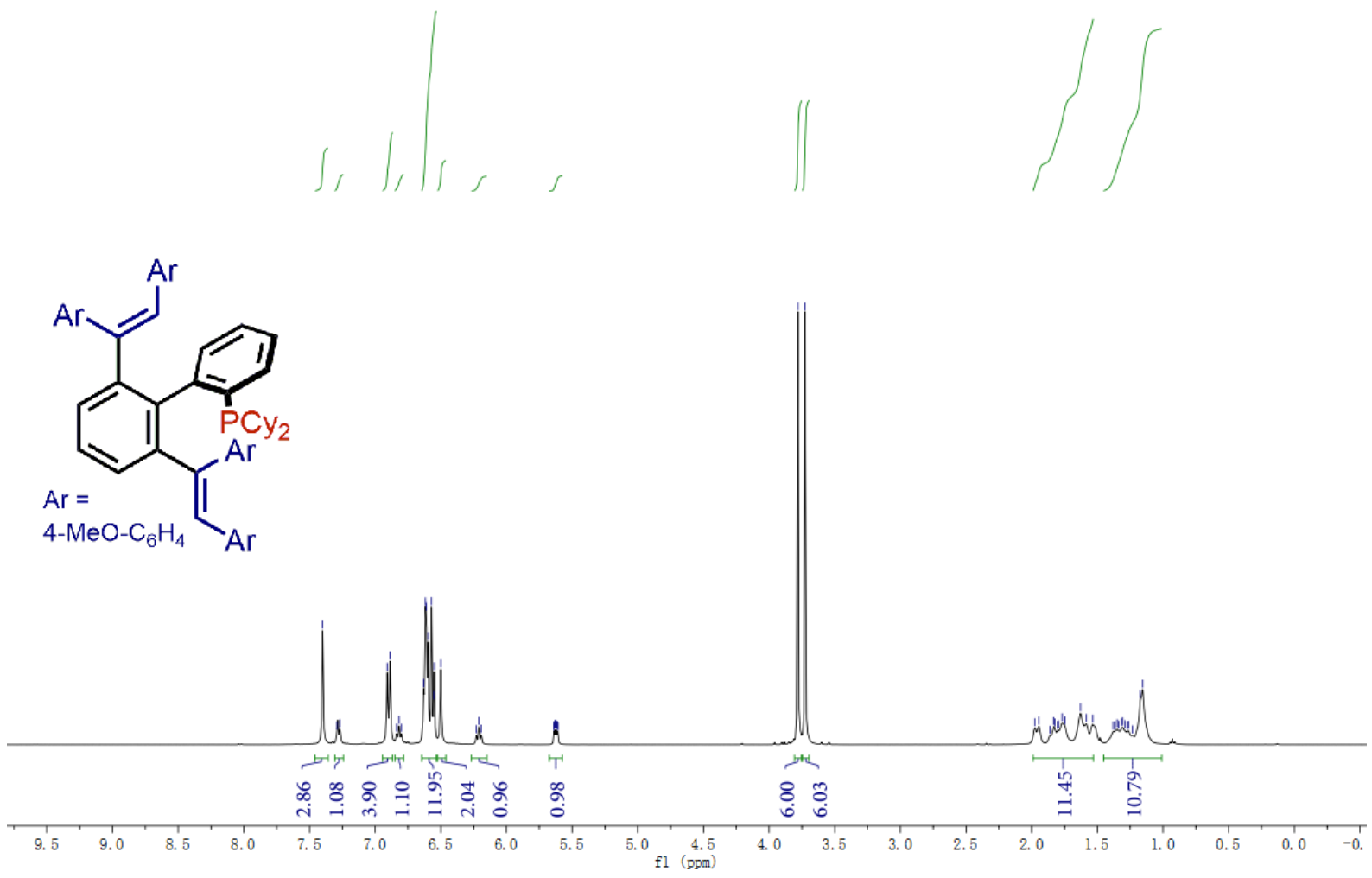

4ad $\mid{ }^{13} \mathrm{C}\left\{{ }^{1} \mathrm{H}\right\} \operatorname{NMR}\left(\mathrm{CDCl}_{3}, 101 \mathrm{MHz}\right)$

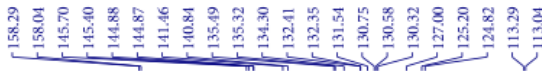
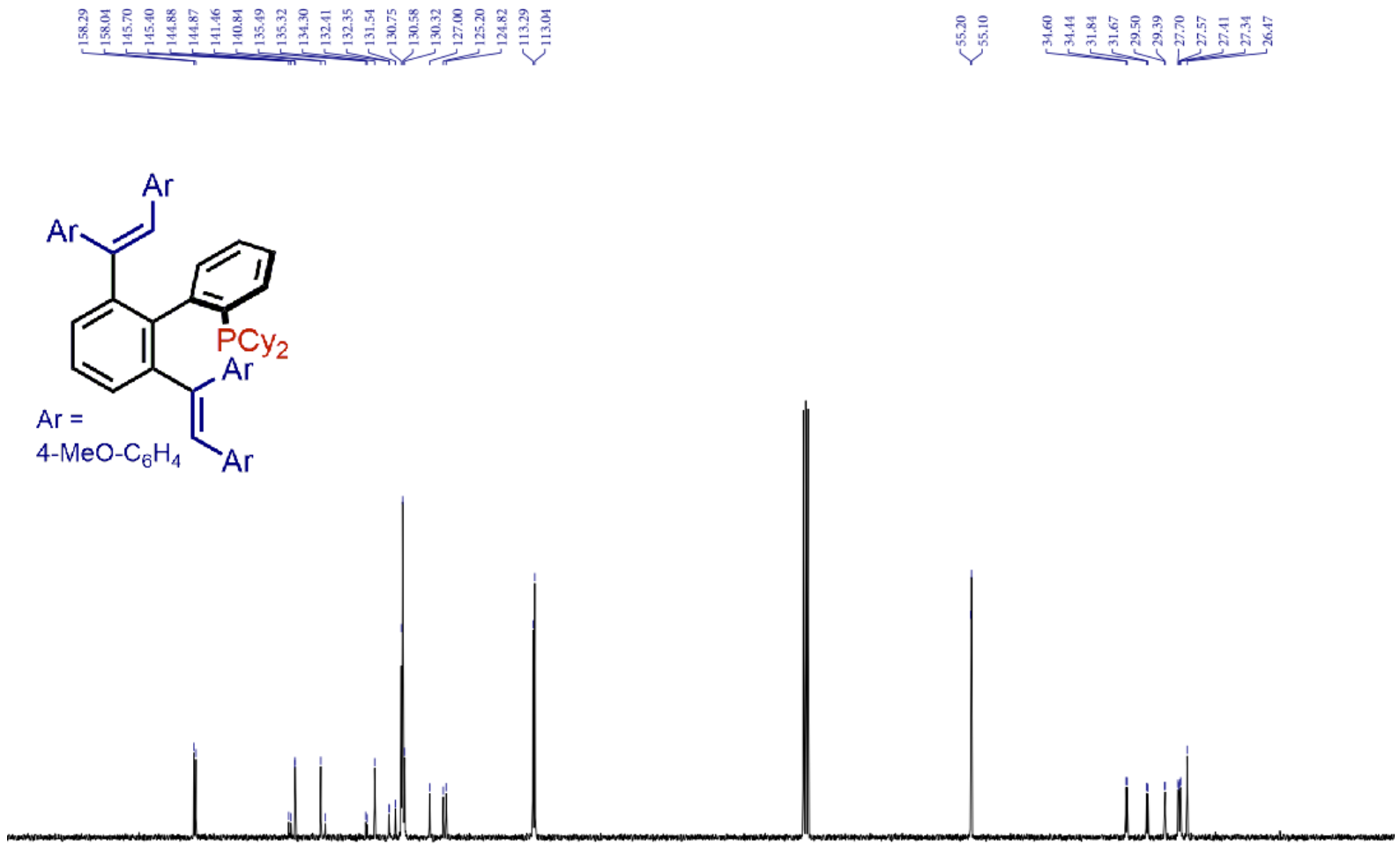

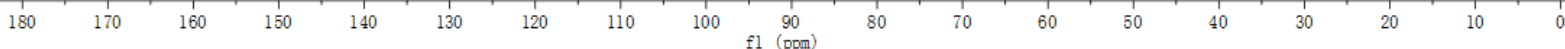


4ad | ${ }^{31} \mathrm{P}\left\{{ }^{1} \mathrm{H}\right\} \mathrm{NMR}\left(\mathrm{CDCl}_{3}, 162 \mathrm{MHz}\right)$

$\vec{\infty}$<smiles>CCOc1ccccc1-c1c(/C(Br)=C/Br)cccc1/C(Br)=C/Br</smiles>

$\begin{array}{rlllllllllllllllllllllllllllllll}140 & 130 & 120 & 110 & 100 & 90 & 80 & 70 & 60 & 50 & 40 & 30 & 20 & 10 & 0 & -10 & -20 & -30 & -40 & -50 & -60 & -70 & -80 & -90 & -100 & -110 & -120 & -130 & -140 & -150\end{array}$ 

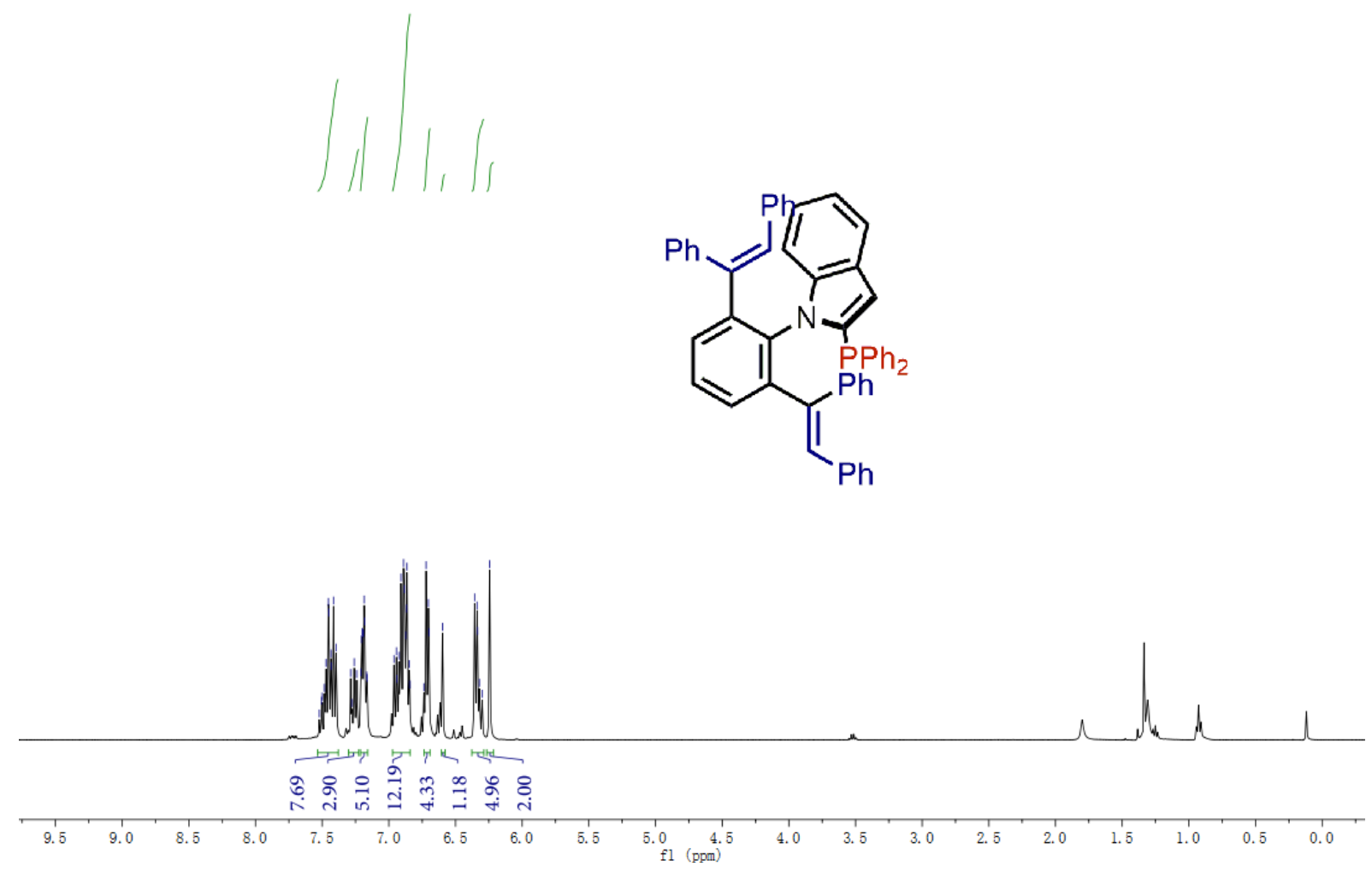

4ja | ${ }^{13} \mathrm{C}\left\{{ }^{1} \mathrm{H}\right\}$ NMR $\left(\mathrm{CDCl}_{3}, 101 \mathrm{MHz}\right)$

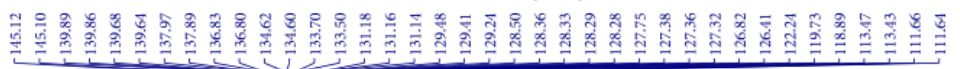

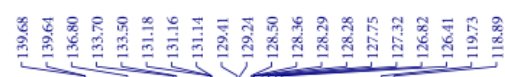
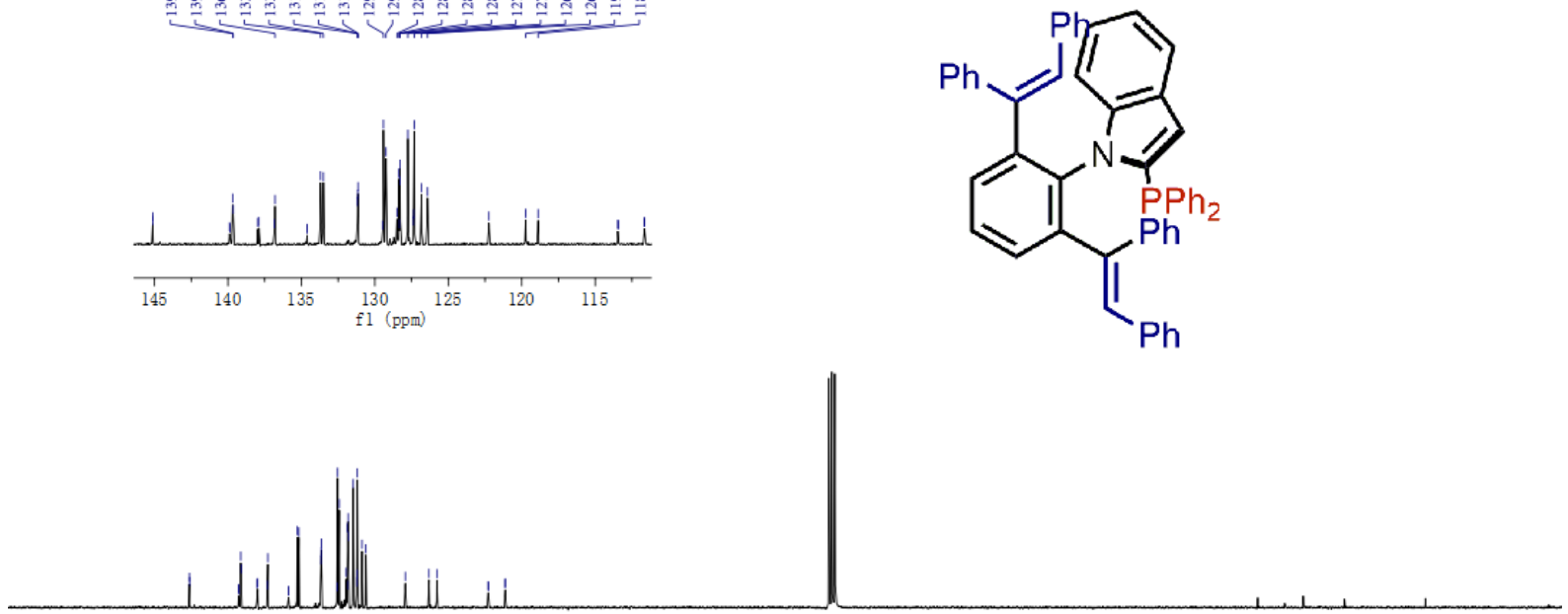

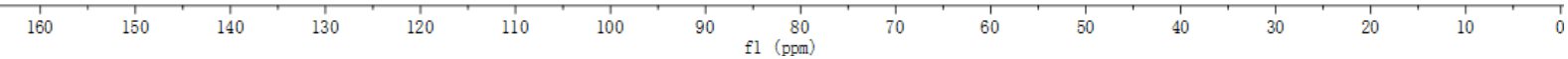


4ja | ${ }^{31} \mathrm{P}\left\{{ }^{1} \mathrm{H}\right\} \mathrm{NMR}\left(\mathrm{CDCl}_{3}, 162 \mathrm{MHz}\right)$<smiles>Pc1cccc(/C(=C\c2ccccc2)c2cccc3cc(-c4ccccc4)n(-c4ccccc4)c23)c1</smiles>

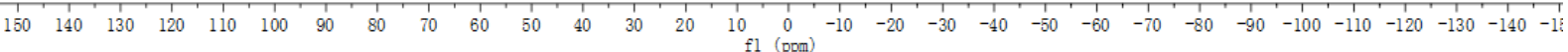


6a $\mid{ }^{1} \mathrm{H} \mathrm{NMR}\left(\mathrm{CDCl}_{3}, 400 \mathrm{MHz}\right)$

然

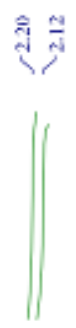

$\overbrace{N}^{\infty}$
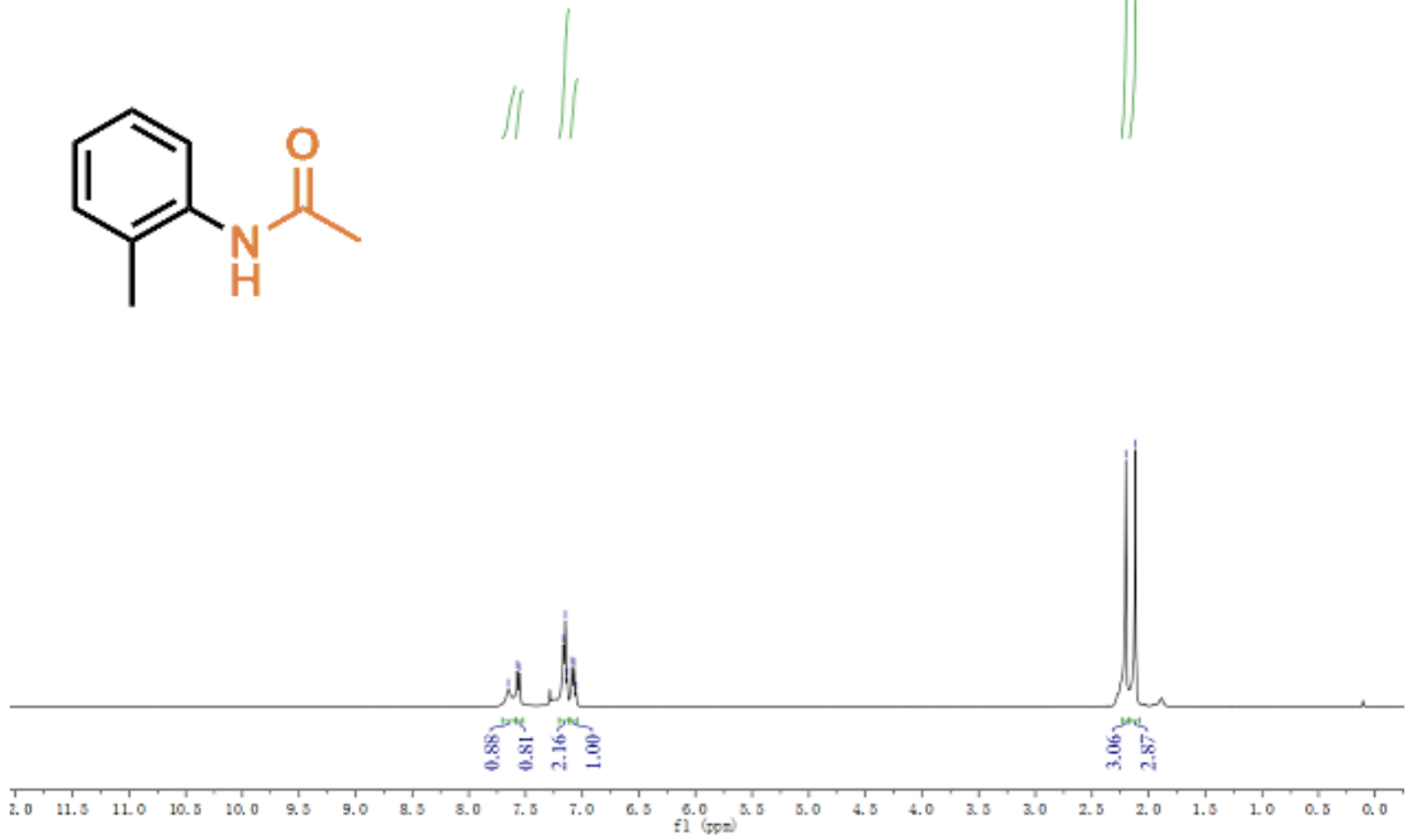

6a $\mid{ }^{13} \mathrm{C}\left\{{ }^{1} \mathrm{H}\right\} \mathrm{NMR}\left(\mathrm{CDCl}_{3}, 101 \mathrm{MHz}\right)$

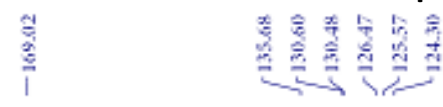

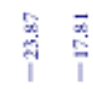
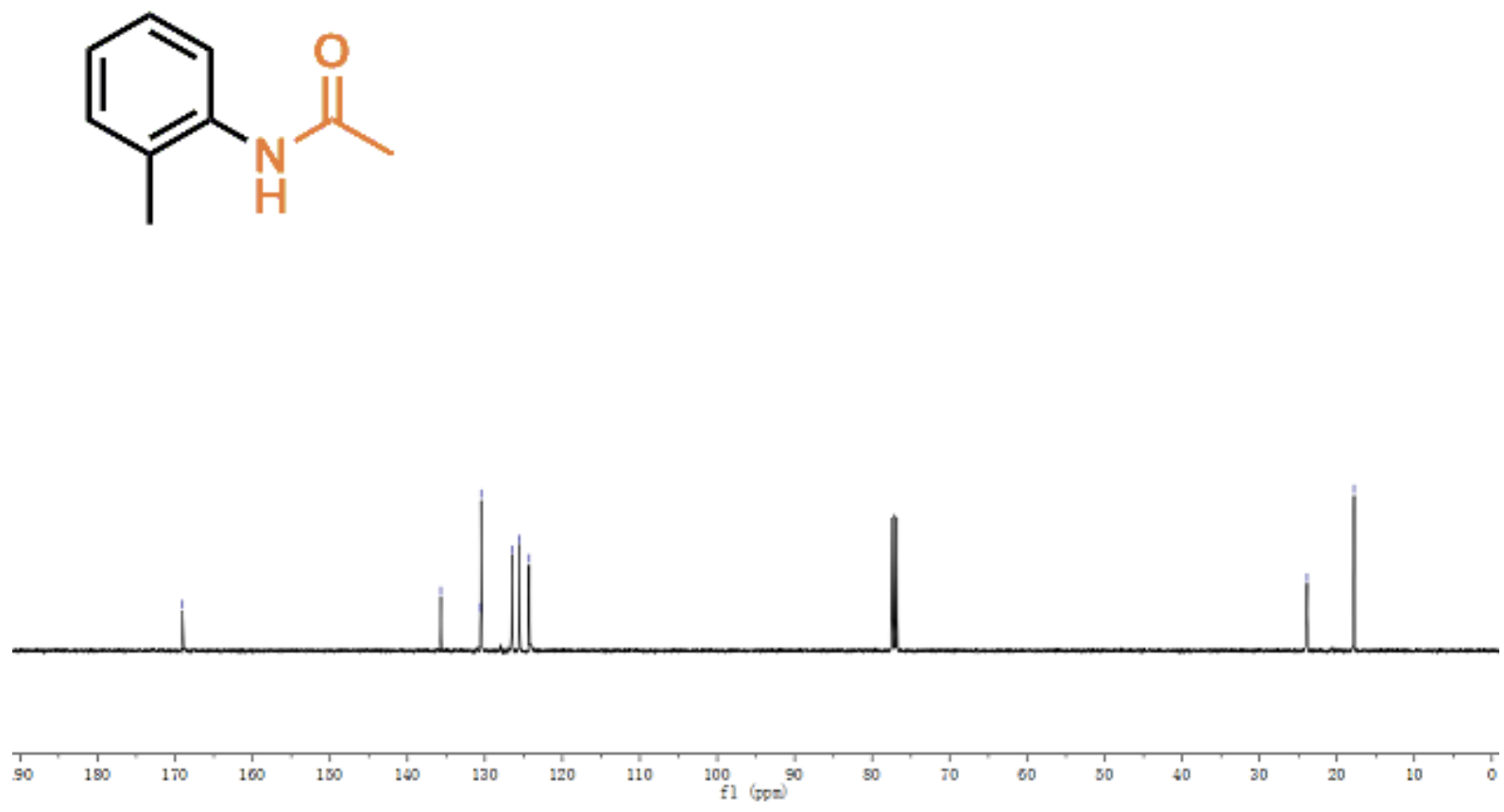

$S-120$ 


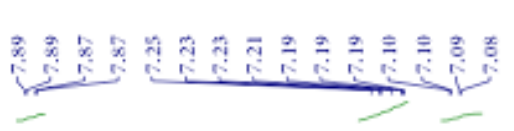<smiles>Cc1ccccc1NC(=O)C(C)(C)C</smiles>
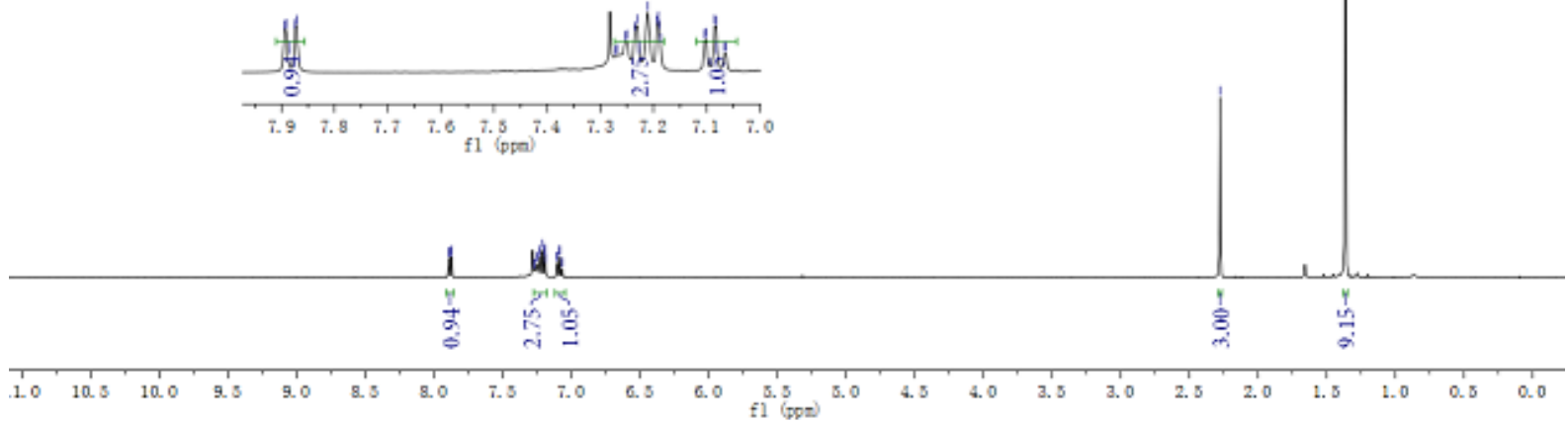

6b $\mid{ }^{13} \mathrm{C}\left\{{ }^{1} \mathrm{H}\right\}$ NMR $\left(\mathrm{CDCl}_{3}, 101 \mathrm{MHz}\right)$<smiles>Cc1ccccc1NC(=O)C(C)(C)C</smiles> 
6c| ${ }^{1} \mathrm{H} \mathrm{NMR}\left(\mathrm{CDCl}_{3}, 400 \mathrm{MHz}\right)$

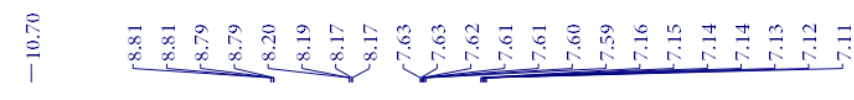<smiles>CC(C)(C)C(=O)Nc1ccccc1[N+](=O)[O-]</smiles>

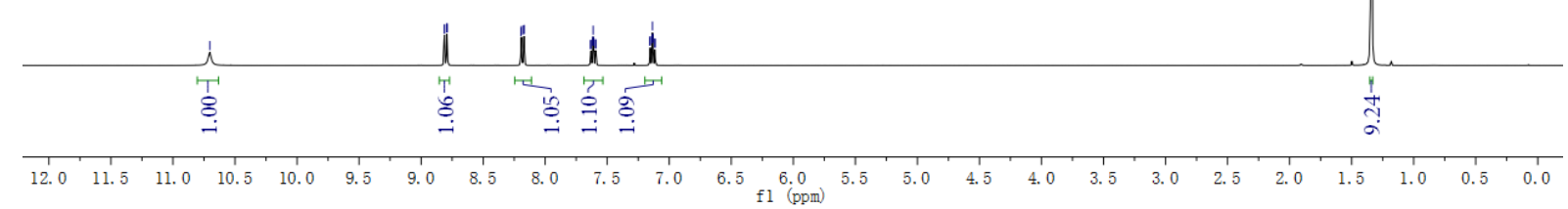

6c $\mid{ }^{13} \mathrm{C}\left\{{ }^{1} \mathrm{H}\right\} \mathrm{NMR}\left(\mathrm{CDCl}_{3}, 101 \mathrm{MHz}\right)$
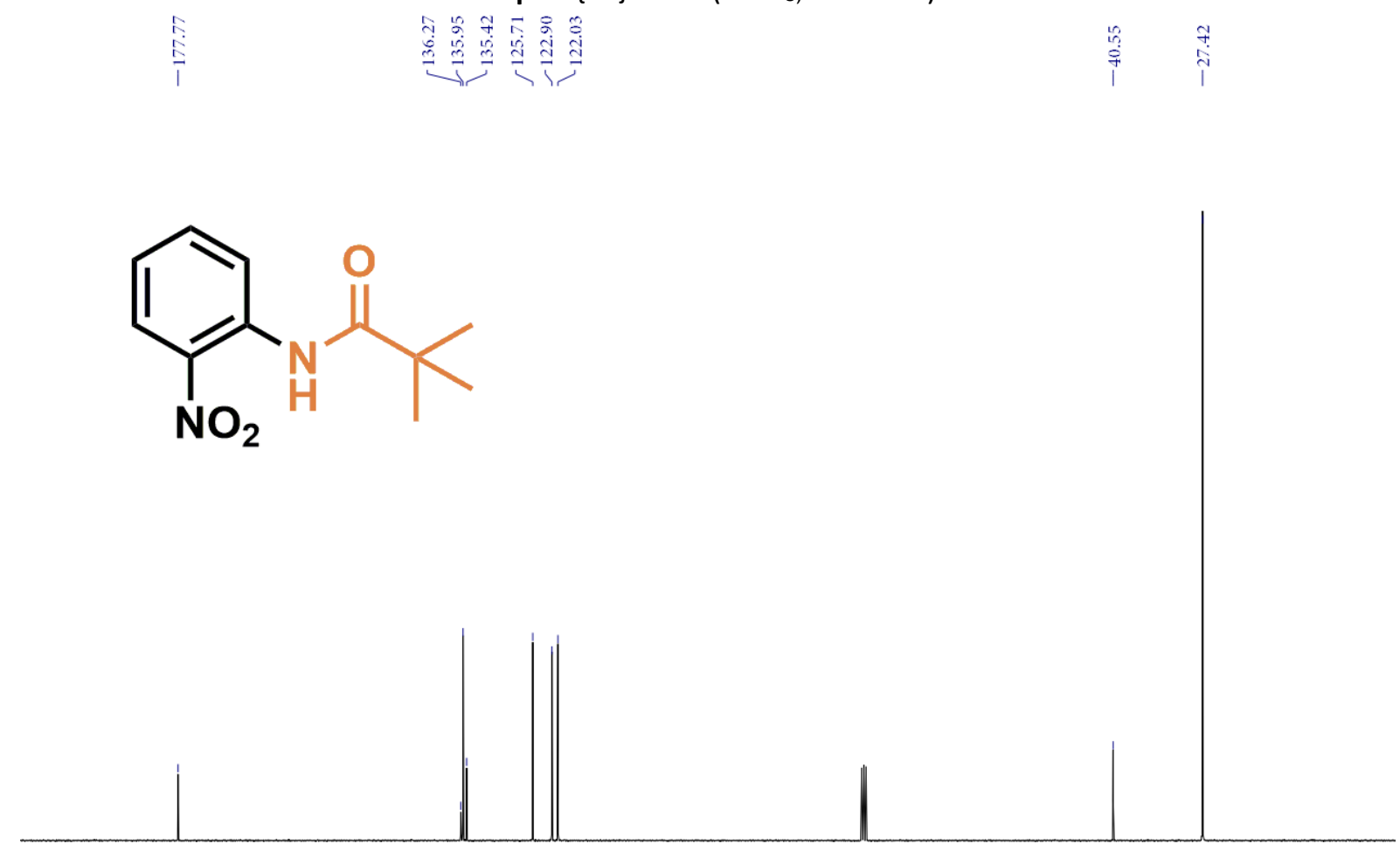

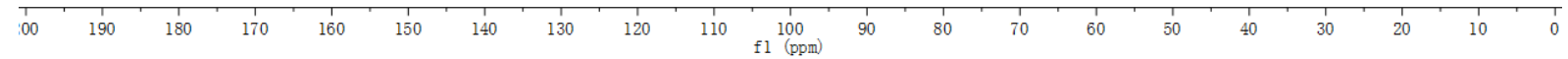


6d | ${ }^{1} \mathrm{H} \mathrm{NMR}\left(\mathrm{CDCl}_{3}, 400 \mathrm{MHz}\right)$

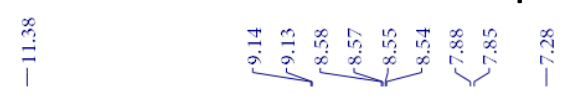
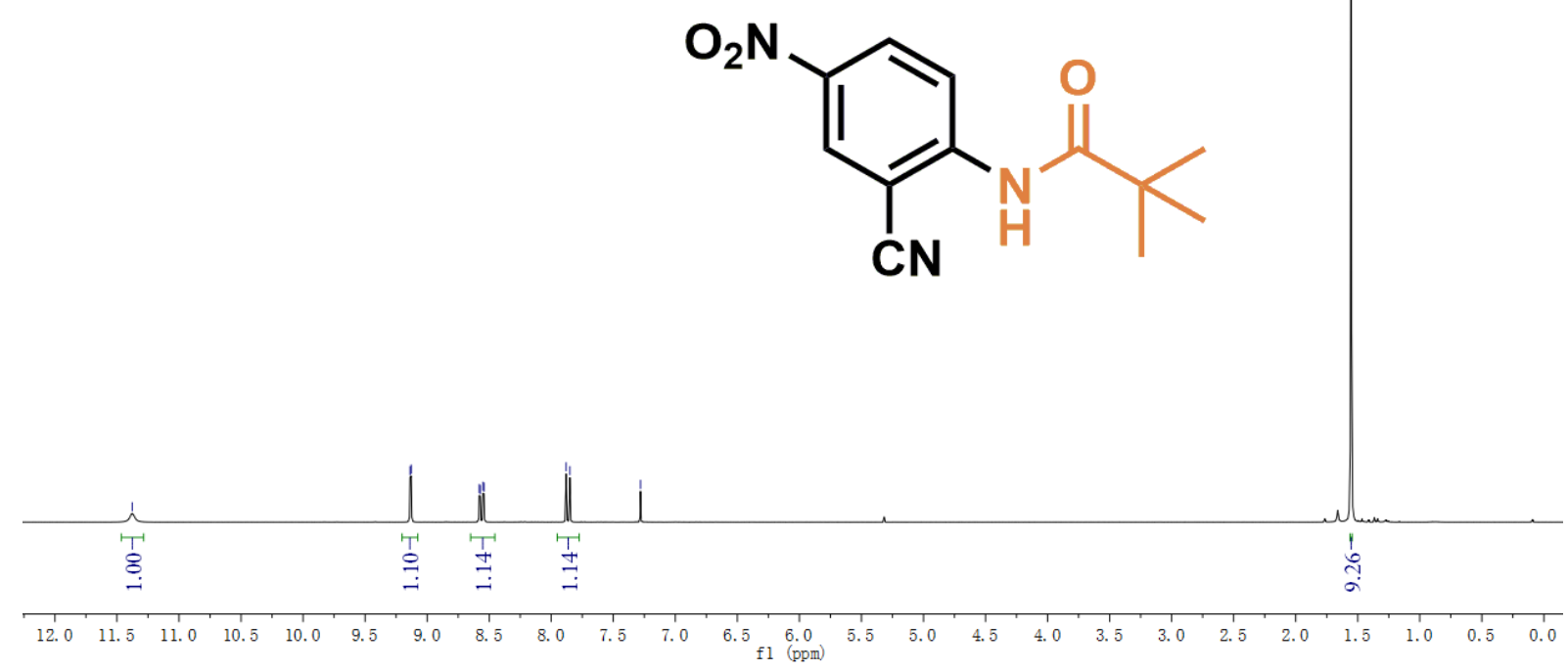

6d | ${ }^{13} \mathrm{C}\left\{{ }^{1} \mathrm{H}\right\} \mathrm{NMR}\left(\mathrm{CDCl}_{3}, 101 \mathrm{MHz}\right)$

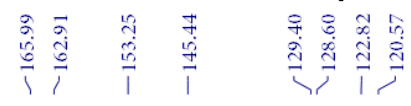

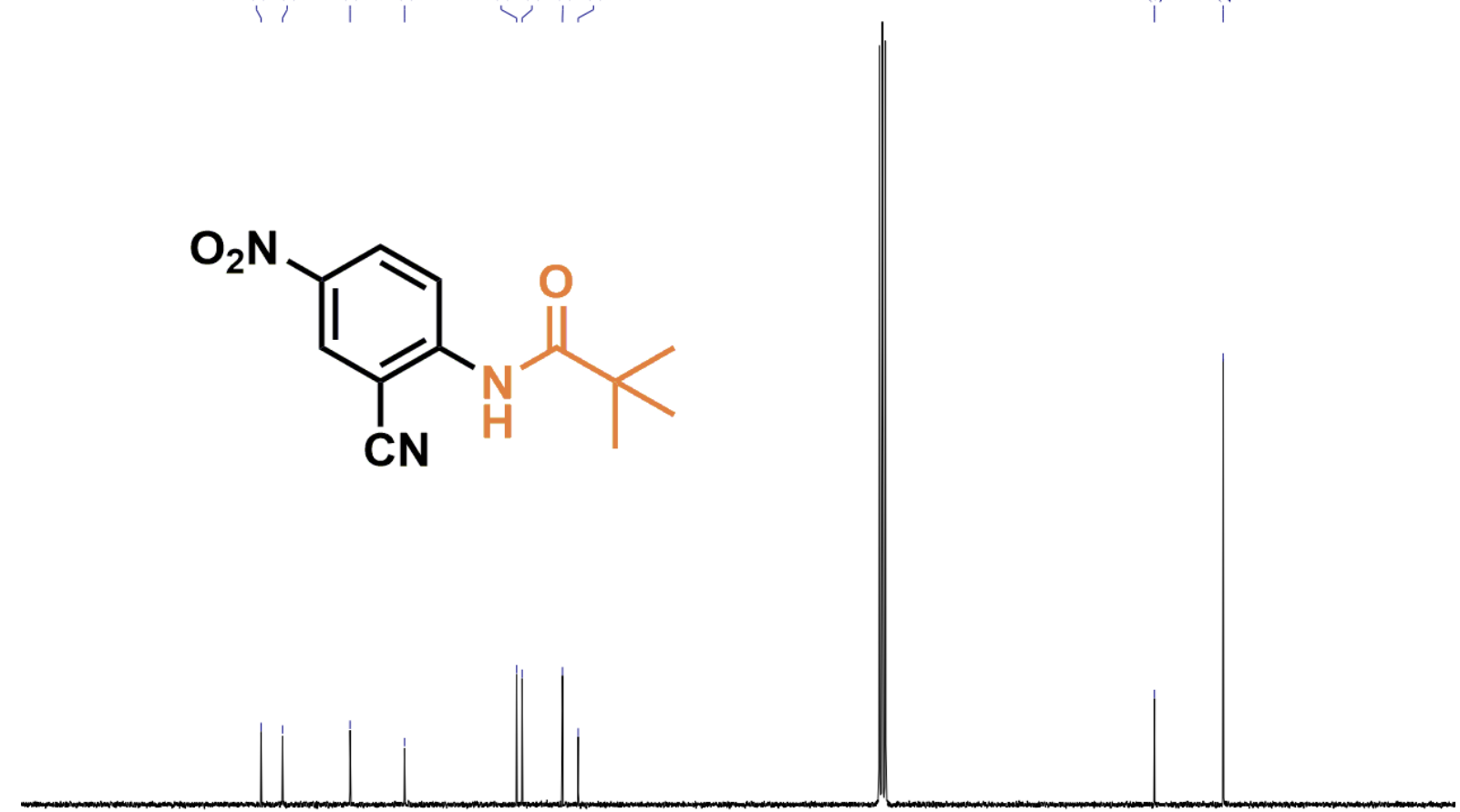

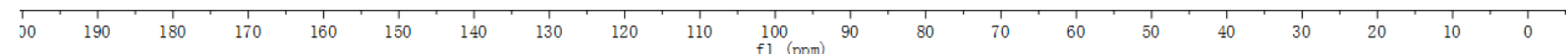

$S-123$ 
6e $\mid{ }^{1} \mathrm{H} \mathrm{NMR}\left(\mathrm{CDCl}_{3}, 400 \mathrm{MHz}\right)$

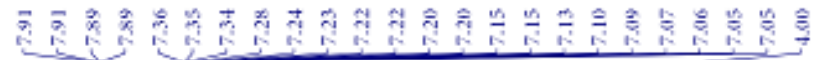

$\int|d| \mid$<smiles>Cc1ccccc1NC(=O)Cc1cccs1</smiles>

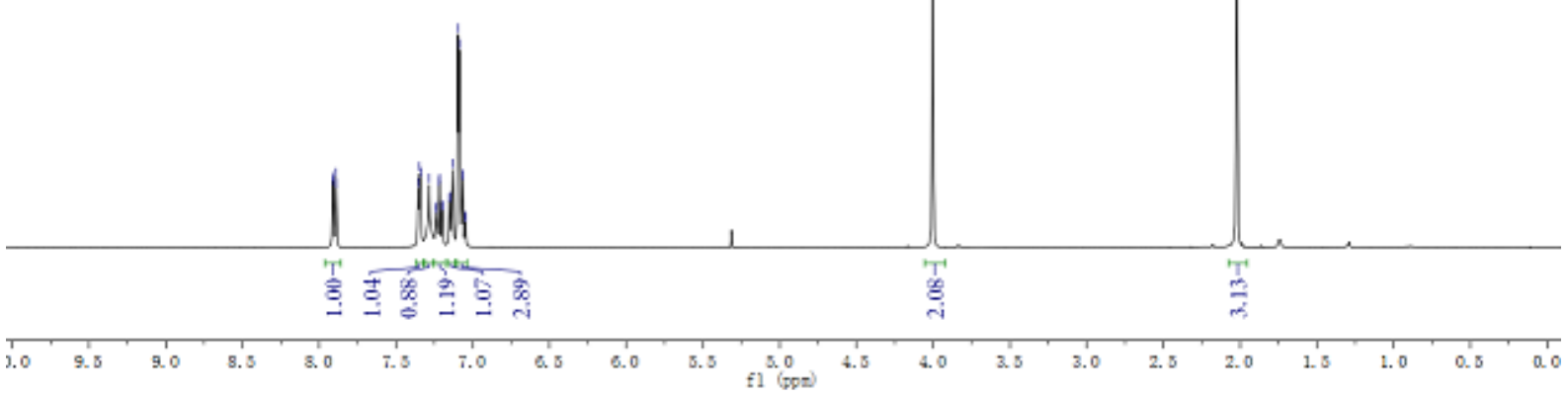

6e $\mid{ }^{13} \mathrm{C}\left\{{ }^{1} \mathrm{H}\right\}$ NMR $\left(\mathrm{CDCl}_{3}, 101 \mathrm{MHz}\right)$

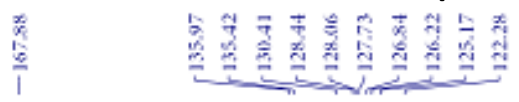<smiles>Cc1ccccc1NC(=O)Cc1cccs1</smiles>

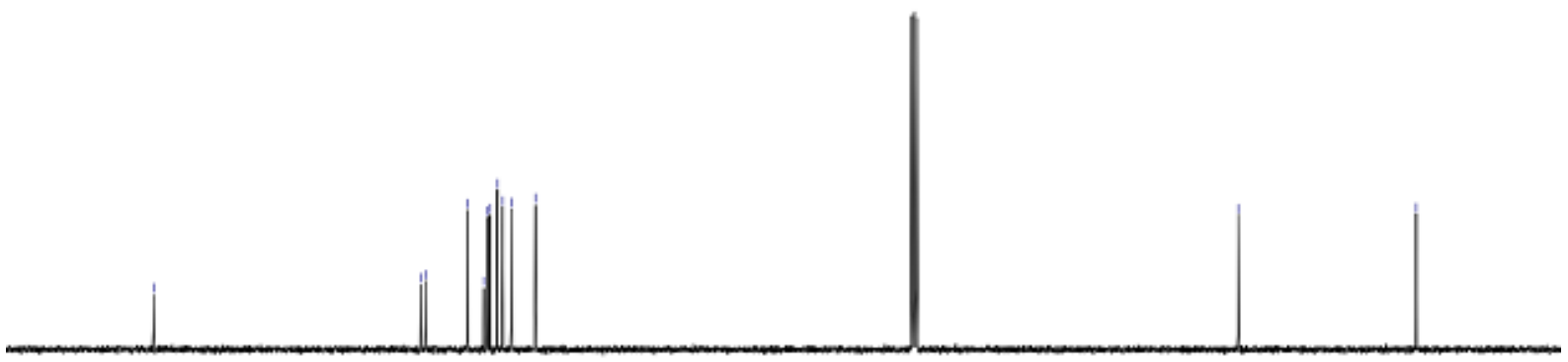

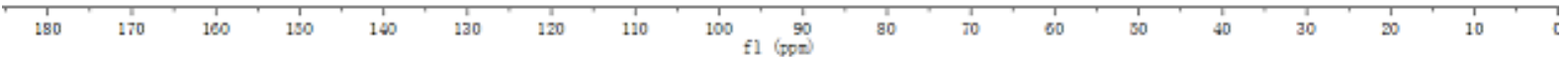


6f | ${ }^{1} \mathrm{H}$ NMR $\left(\mathrm{CDCl}_{3}, 400 \mathrm{MHz}\right)$

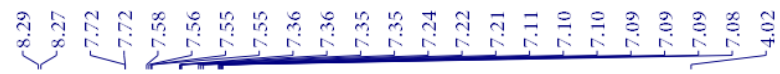
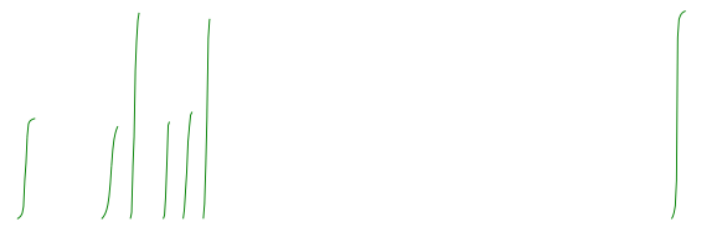<smiles>O=C(Cc1cccs1)Nc1ccccc1C(F)(F)F</smiles>

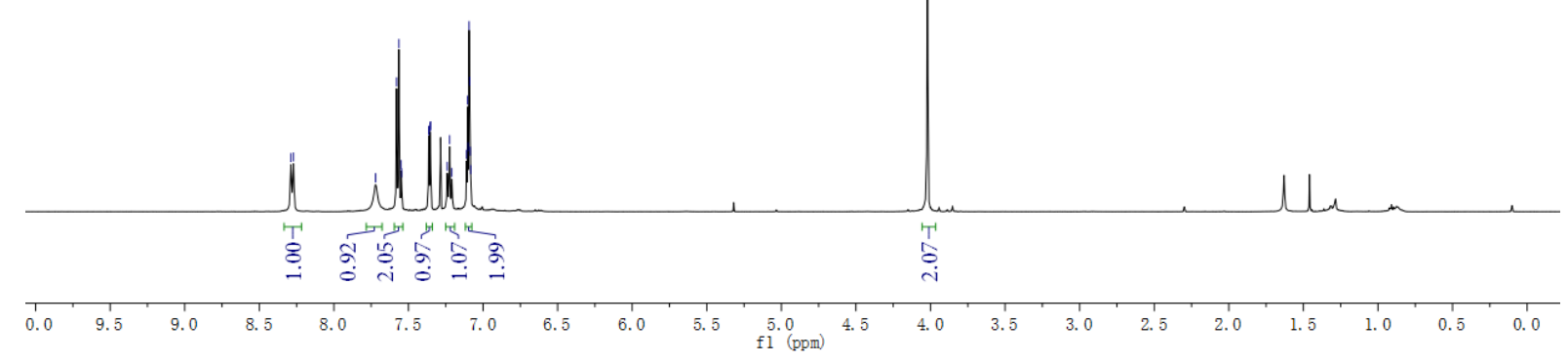

6f $\mid{ }^{13} \mathrm{C}\left\{{ }^{1} \mathrm{H}\right\} \mathrm{NMR}\left(\mathrm{CDCl}_{3}, 101 \mathrm{MHz}\right)$

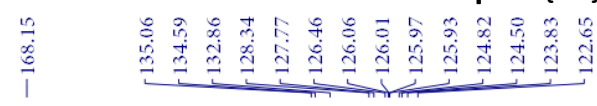<smiles>O=C(Cc1cccs1)Nc1ccccc1C(F)(F)F</smiles>

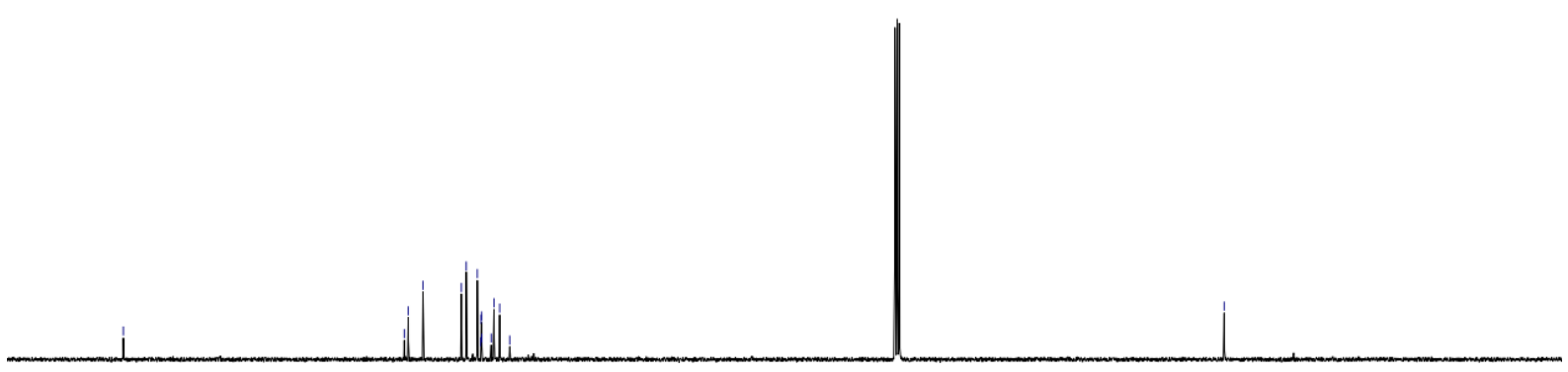

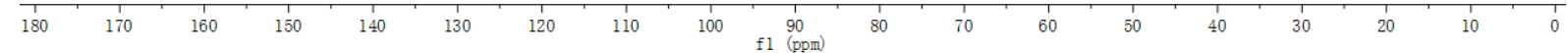


6f ${ }^{19} \mathrm{~F}\left\{{ }^{1} \mathrm{H}\right\} \mathrm{NMR}\left(\mathrm{CDCl}_{3}, 471 \mathrm{MHz}\right)$

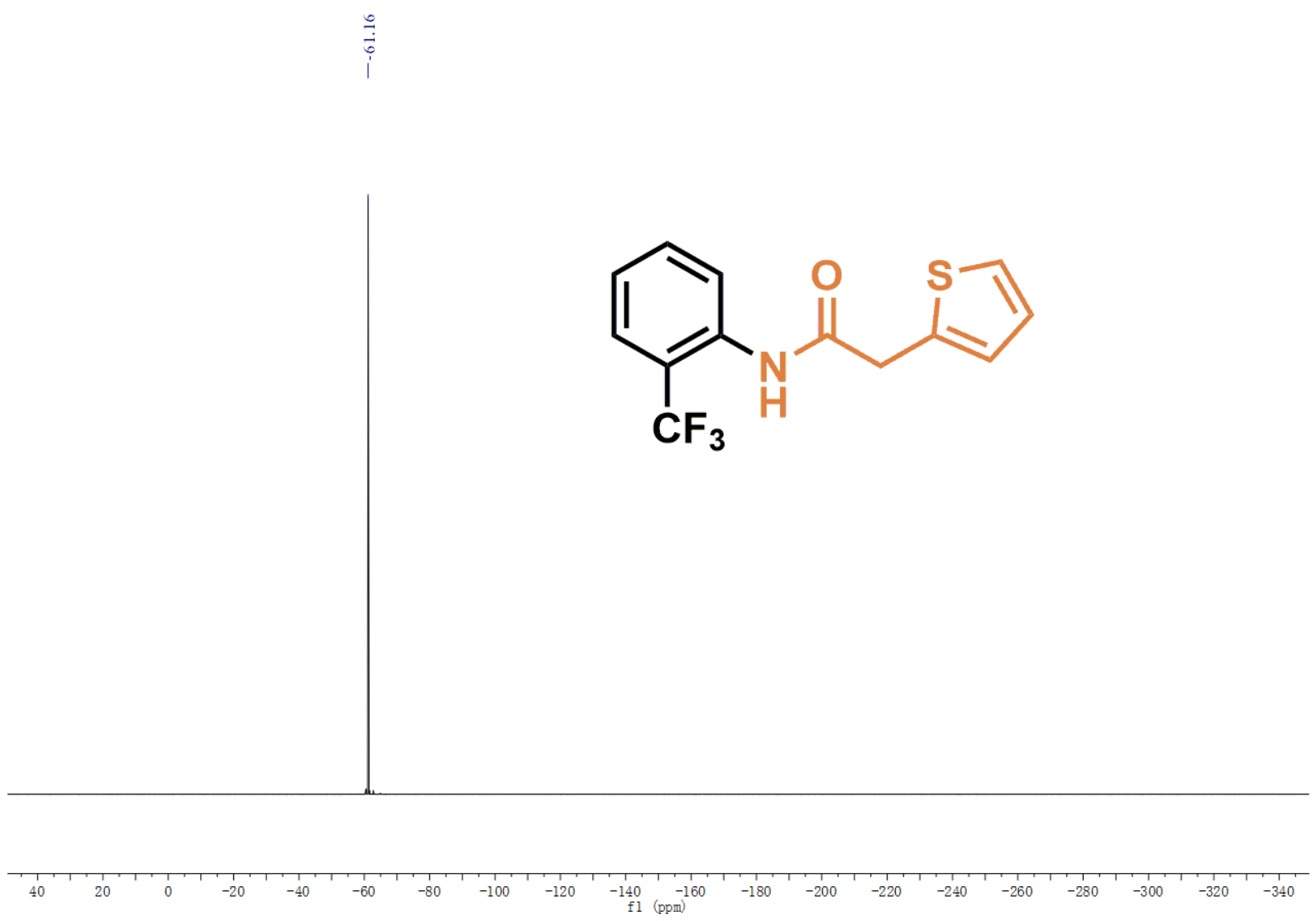




\section{References}

[1] J. Chatt, L. M. Venanzi, Journal of the Chemical Society (Resumed) 1957, 4735-4741.

[2] Martinez, A. M.; Echavarren, J.; Alonso, I.; Rodriguez, N.; Gomez Arrayas, R.; Carretero, J. C., Chemical science 2015, 6 (10), 5802-5814.

[3] Fors, B. P.; Dooleweerdt, K.; Zeng, Q.; Buchwald, S. L., Tetrahedron 2009, 65 (33), 6576-6583.

[4] Brasche, G.; Garcia-Fortanet, J.; Buchwald, S. L., Organic letters 2008, 10 (11), 2207-2210.

[5] Lourenço, M. C. S.; Vicente, F. R.; de Oliveira, M. d. G. M.; Candéa, A. L. P.; Gonçalves, R. S. B.; Nogueira, T. C. M.; de Lima Ferreira, M.; de Souza, M. V. N., Bioorganic \& medicinal chemistry letters 2007, 17 (24), 6895-6898. 